MARCELO COLPAS DA SILVA

APLICAÇÃO DO MÉTODO DA OTIMIZAÇÃO TOPOLÓGICA PARA O PROJETO DE MECANISMOS FLEXÍVEIS MENOS SUSCETÍVEIS À OCORRÊNCIA DE DOBRADIÇAS 
MARCELO COLPAS DA SILVA

\section{APLICAÇÃO DO MÉTODO DA OTIMIZAÇÃO TOPOLÓGICA PARA O PROJETO DE MECANISMOS FLEXÍVEIS MENOS SUSCETÍVEIS À OCORRÊNCIA DE DOBRADIÇAS}

Dissertação apresentada à Escola Politécnica da Universidade de São Paulo para a obtenção do título de Mestre em Engenharia 


\section{APLICAÇÃO DO MÉTODO DA OTIMIZAÇÃO TOPOLÓGICA PARA O PROJETO DE MECANISMOS FLEXÍVEIS MENOS SUSCETÍVEIS À OCORRÊNCIA DE DOBRADIÇAS}

Dissertação apresentada à Escola Politécnica da Universidade de São Paulo para a obtenção do título de Mestre em Engenharia

Área de Concentração:

Engenharia Mecânica e Mecatrônica

Orientador:

Prof. Dr. Emílio Carlos Nelli Silva 
Este exemplar foi revisado e alterado em relação à versão original, sob responsabilidade única do autor e com a anuência de seu orientador.

São Paulo, 18 de junho de 2007.

Marcelo Colpas da Silva

(autor)

Prof. Dr. Emílio Carlos Nelli Silva

(orientador)

FICHA CATALOGRÁFICA

Silva, Marcelo Colpas da

Aplicação do método da otimização topológica para o proje to de mecanismos flexíveis menos suscetíveis à ocorrência de dobradiças / M.C. da Silva. -- São Paulo, 2007. 162 p.

Dissertação (Mestrado) - Escola Politécnica da Universidade de São Paulo. Departamento de Engenharia Mecatrônica e de Sistemas Mecânicos.

1.Topologia (Otimização) 2.Mecanismos 3.Sistemas microeletromecânicos 4.Método dos elementos finitos I.Universidade de São Paulo. Escola Politécnica. Departamento de Engenharia Mecatrônica e de Sistemas Mecânicos Il.t. 
À minha esposa Regina e ao meu primogênito Eduardo. Pelo estímulo e motivação à conclusão deste trabalho. 


\section{AGRADECIMENTOS}

Ao Professor Emílio, pela confiança que demonstrou ao me conceder a oportunidade de ser seu orientado.

Aos meus pais, Olavo e Eunice, pelo apoio concreto em todas as etapas da minha vida.

Ao meu irmão, Fernando, e ao meu grande amigo, Oliveira, pelo carinho de suas palavras nos momentos de dúvida.

E, em especial, à Regina, pela dedicação e compreensão incondicionais, frutos do amor que temos um pelo outro. 


\section{RESUMO}

Os mecanismos flexíveis são dispositivos capazes de transmitir força e movimento através da deformação elástica. Têm grande importância a uma série de aplicações nas quais os mecanismos de corpos rígidos não seriam viáveis, como por exemplo, os sistemas microeletromecânicos. Existem várias maneiras pelas quais os mecanismos flexíveis podem ser projetados, sendo a otimização topológica um método bastante difundido por ser de aplicação sistemática, ou seja, não requer do projetista qualquer ação analítica durante a etapa de projeto. Na maioria dos casos, o método da otimização topológica combina o método dos elementos finitos com um método de programação matemática. Logo, faz-se necessário discretizar a região do espaço na qual o material disponível será distribuído para determinar o mecanismo flexível adequado à aplicação desejada. Freqüentemente, o mecanismo projetado apresenta duas regiões sólidas unidas por um único nó pertencente à malha de elementos finitos. Durante a transmissão do movimento, este nó age como uma dobradiça conectada às duas regiões. Trata-se de um efeito indesejado, pois compromete a modelagem e a fabricação do componente mecânico. Assim, neste trabalho, foram estudadas técnicas destinadas à redução da ocorrência das “dobradiças” no projeto de mecanismos flexíveis por otimização topológica. Foi implementado em linguagem $\mathrm{C}$ um código que permite projetar mecanismos flexíveis submetidos a um único carregamento ou múltiplos carregamentos (mecanismos multi-flexíveis). Com o objetivo de analisar e explorar outros aspectos da formulação implementada no código, investigou-se também a sua utilização no projeto de estruturas rígidas. Como resultado, é mostrada a influência dos diversos parâmetros de otimização no projeto de mecanismos flexíveis sem dobradiças, permitindo analisar a eficácia da formulação implementada.

Palavras chave: Otimização topológica, Mecanismos flexíveis, Dobradiças, Otimização estrutural, Elementos finitos. 


\begin{abstract}
Compliant mechanisms are devices capable of transmitting force and displacement through elastic deformation. They are extremely important for a number of applications in which the mechanisms of rigid bodies would not be feasible, such as microelectromechanical systems. There are several ways through which compliant mechanisms can be designed, being topology optimization a highly diffused method because of its systematic application, once, it does not require from the designer any analytical action during the stage of the project. In most cases, topology optimization method combines the finite element method with a mathematical program method. Therefore, it is necessary to discretize the region of the space in which the available material will be distributed to determine the appropriate compliant mechanism for the desired application. However, the mechanism designed often presents two solid regions united by one single node. During movement transmission, this node acts as a hinge connected to both regions. This is an undesired effect, as it compromises the modeling and manufacturing of the mechanical component. Thus, this work covers techniques aiming at reducing the occurrence of hinges in the design of compliant mechanisms through topology optimization. A code in C language was implemented, which allows the design of compliant mechanisms subjected to one single load or multiple loads (multi-compliant mechanisms). With the purpose of analyzing and exploring other aspects of the formulation implemented in the code, its use in the design of rigid structures was also investigated. As a result, the influence of several optimization parameters in the design of compliant mechanisms without hinges is shown. This allows to analyze the efficiency of the formulation implemented.
\end{abstract}

Key words: Topology optimization, Compliant mechanisms, Hinges, Structural optimization, Finite elements. 


\section{LISTA DE FIGURAS}

Figura 1.1 - (a) mecanismo formado por corpos rígidos; (b) mecanismo equivalente com os pivôs flexíveis.

Figura 1.2 - (a) mecanismo de pseudo-corpo rígido; (b) mecanismo flexível equivalente.

Figura 1.3 - (a) domínio de projeto; (b) quantidade limitada de material sendo distribuida no domínio de projeto; (c) resultado da otimização topológica; (d) configuração deformada do mecanismo sintetizado 23

Figura 2.1 - Elemento isoparamétrico de quatro nós. 27

Figura 2.2 - Principais abordagens empregadas na otimização estrutural. 34

Figura 2.3 - Etapas relacionadas ao método da otimização topológica 35

Figura 2.4 - Representação de uma estrutura resultante dos trabalhos de Michell........... 36

Figura 2.5 - Exemplo de distribuição de material segundo a parametrização discreta..... 38

Figura 2.6 - (a) representação da metade de uma viga simétrica obtida a partir de uma discretização de 800 elementos finitos; (b) novo resultado obtido a partir de uma discretização de 1800 elementos finitos.

Figura 2.7 - Representação esquemática de uma distribuição de material através da homogeneização

Figura 2.8 - Ocorrência da instabilidade de tabuleiro. 45

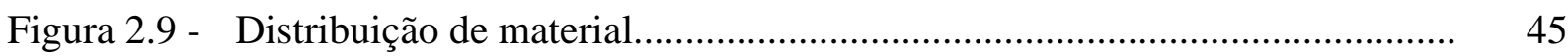

Figura 2.10 - Mola substituindo a peça de trabalho no ponto B........................................ 47

Figura 2.11 - Carregamentos distintos em um mesmo corpo........................................... 50

Figura 2.12 - Falta de conectividade entre duas regiões da topologia............................... 51

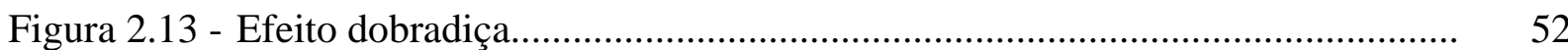

Figura 2.14 - (a) ocorrência do efeito dobradiça; (b) pescoço obtido por otimização de forma.

Figura 2.15 - Teste da “monotonicidade” na região delimitada pela circunferência de centro $\mathrm{O}$

Figura 2.16 - Teste de "monotonicidade” para três elementos finitos de um grupo de quatro elementos.

Figura 2.17 - Possíveis valores para h. 
Figura 2.18 - Comportamento da energia de deformação específica.

Figura 2.19 - Domínio fixo do problema de otimização submetido ao carregamento externo

Figura 2.20 - Comportamento da energia de deformação específica penalizada 61

Figura 2.21 - Domínio fixo submetido a c carregamentos distintos. 64

Figura 2.22 - Domínio fixo discretizado em $\mathrm{N}$ elementos finitos. 68

Figura 2.23 - Aplicação de c carregamentos sobre o domínio fixo de projeto discretizado em $\mathrm{N}$ elementos finitos 69

Figura 3.1 - Gráfico da função objetivo $F(x)$ 81

Figura 3.2 - Gráfico da função restringida $F(x)$ 84

Figura 3.3 - Elemento central i sofrendo a influência dos elementos adjacentes...... 88

Figura 3.4 - Redução da área de influência do filtro em relação ao elemento finito i...... 89

Figura 3.5 - Elemento central i sofrendo a influência dos demais elementos cujos centróides estão no interior da circunferência.

Figura 3.6 - Fluxo de dados do código computacional para o projeto de mecanismos flexíveis

Figura 4.1 - Representação do problema de otimização. 95

Figura 4.2 - Topologia obtida a partir de $\rho_{\text {einicial }}=0,001$. 96

Figura 4.3 - Topologia obtida a partir de $\rho_{\text {einicial }}=0,500$ 96

Figura 4.4 - Topologia obtida a partir de $\rho_{\text {einicial }}=1,000$.

Figura 4.5 - Topologia obtida a partir de valores aleatórios atribuídos às variáveis de projeto.

Figura 4.6 - Representação do problema de otimização 98

Figura 4.7 - Topologia obtida com a discretização de 1250 elementos. 99

Figura 4.8 - Topologia obtida com a discretização de 2450 elementos...... 99

Figura 4.9 - Topologia obtida com a discretização de 2888 elementos...... 99

Figura 4.10 - Representação do problema de otimização. 100

Figura 4.11 - Presença da instabilidade de tabuleiro. 101

Figura 4.12 - Topologia obtida a partir de $\rho_{\text {einicial }}=0,001$ e uso do filtro espacial de densidades

Figura 4.13 - Topologia obtida a partir de $\rho_{\text {einicial }}=0,500$ e uso do filtro espacial de densidades 
Figura 4.14 - Topologia obtida a partir de $\rho_{\text {einicial }}=1,000$ e uso do filtro espacial de densidades

Figura 4.15 - Topologia obtida a partir de valores aleatórios atribuídos às variáveis de projeto e uso do filtro espacial de densidades.

Figura 4.16 - Topologia obtida com a discretização de 1250 elementos e uso do filtro espacial de densidades. 104

Figura 4.17 - Gráficos de convergência referentes à topologia da Figura 4.16. 105

Figura 4.18 - Topologia obtida com a discretização de 2450 elementos e uso do filtro espacial de densidades.

Figura 4.19 - Gráficos de convergência referentes à topologia da Figura 4.18. 106

Figura 4.20 - Topologia obtida com a discretização de 2888 elementos e uso do filtro espacial de densidades.

Figura 4.21 - Gráficos de convergência referentes à topologia da Figura 4.20. 107

Figura 4.22 - Topologia obtida com $\mathrm{R}_{\max }=1,05 \mathrm{~mm}$. 108

Figura 4.23 - Gráficos de convergência referentes à topologia obtida com

$$
\mathrm{R}_{\text {max }}=1,05 \mathrm{~mm} \text {. }
$$

Figura 4.24 - Interpretação da solução apresentada na Figura 4.22. 109

Figura 4.25 - Configuração deformada..... 109

Figura 4.26 - Topologia obtida com $\mathrm{R}_{\max }=1,30 \mathrm{~mm}$.

Figura 4.27 - Gráficos de convergência referentes à topologia obtida com

$$
\mathrm{R}_{\max }=1,30 \mathrm{~mm} \text {. }
$$

Figura 4.28 - Topologia obtida com $\mathrm{R}_{\max }=1,60 \mathrm{~mm}$

Figura 4.29 - Gráficos de convergência referentes à topologia obtida com

$$
\mathrm{R}_{\text {max }}=1,60 \mathrm{~mm} \text {. }
$$

Figura 4.30 - Representação do problema de otimização.

Figura 4.31 - Topologia obtida por meio da formulação (2.57).

Figura 4.32 - Topologia obtida por meio do código computacional elaborado por Lima (2002)

Figura 4.33 - Configuração deformada

Figura 4.34 - Mapa de cores representando as tensões segundo o critério de resistência de von Mises (em N/mm²).

Figura 4.35 - Representação do problema de otimização 
Figura 4.36 - Topologia obtida para o problema que considera as forças distribuídas......

Figura 4.37 - Mapa de cores representando as tensões segundo o critério de resistência de von Mises (em N/mm²)

Figura 4.38 - Configuração deformada.

Figura 4.39 - Topologia obtida para o problema cujos deslocamentos horizontais dos pontos de saída foram restringidos

Figura 4.40 - Configuração deformada.

Figura 4.41 - Configuração final do mecanismo flexível

Figura 4.42 - Representação do problema de otimização.

Figura 4.43 - Topologia obtida com restrição de 15\% do volume do domínio fixo

Figura 4.44 - Topologia obtida com restrição de 20\% do volume do domínio fixo.

Figura 4.45 - Topologia obtida com restrição de 25\% do volume do domínio fixo.

Figura 4.46 - Topologia obtida com um raio de abrangência igual a 0,61 mm.

Figura 4.47 - Topologia obtida com restrição de 15\% do volume do domínio fixo 125

Figura 4.48 - Topologia obtida com restrição de 20\% do volume do domínio fixo 126

Figura 4.49 - Topologia obtida com restrição de 25\% do volume do domínio fixo 126

Figura 4.50 - Topologia obtida com um raio de abrangência igual a 0,70 mm. 127

Figura 4.51 - Topologia obtida com restrição de 15\% do volume do domínio fixo 128

Figura 4.52 - Topologia obtida com restrição de 20\% do volume do domínio fixo 128

Figura 4.53 - Topologia obtida com restrição de 25\% do volume do domínio fixo. 128

Figura 4.54 - Configuração deformada do mecanismo obtido com restrição volumétrica de $15 \%$

Figura 4.55 - Configuração deformada do mecanismo obtido com restrição volumétrica de $20 \%$.

Figura 4.56 - Configuração deformada do mecanismo obtido com restrição volumétrica de $25 \%$

Figura 4.57 - Distribuição da energia de deformação específica (em N/mm²) com restrição volumétrica de $15 \%$.

Figura 4.58 - Distribuição da energia de deformação específica (em N/mm²) com restrição volumétrica de $20 \%$.

Figura 4.59 - Distribuição da energia de deformação específica (em N/mm²) com restrição volumétrica de $25 \%$.

Figura 4.60 - Representação do problema de otimização. 
Figura 4.61 - Topologia para compressão mínima da mola equivalente a 1mm e

$$
\mathrm{k}_{\mathrm{b}}=50 \mathrm{~N} / \mathrm{mm}
$$

Figura 4.62 - Topologia para compressão mínima da mola equivalente a 0,5 mm e

$$
\mathrm{k}_{\mathrm{b}}=50 \mathrm{~N} / \mathrm{mm}
$$

Figura 4.63 - Topologia para compressão mínima da mola equivalente a 0,5 mm e

$$
\mathrm{k}_{\mathrm{b}}=22,5 \mathrm{~N} / \mathrm{mm}
$$

Figura 4.64 - Topologia obtida para o problema sem mola e sem ponto de saída

Figura 4.65 - Representação do problema de otimização.

Figura 4.66 - (a) solução do problema de otimização topológica; (b) mapa de cores indicando as tensões segundo o critério de resistência de von Mises (em $\left.\mathrm{N} / \mathrm{mm}^{2}\right)$

Figura 4.67 - (a) topologia obtida para $n=2$; (b) topologia obtida para $n=4$

Figura 4.68 - (a) solução do problema de otimização topológica com estimativa inicial de 0,15; (b) mapa de cores indicando as tensões segundo o critério de resistência de von Mises (em N/mm²).

Figura 4.69 - (a) solução do problema de otimização topológica com estimativa inicial de 0,50; (b) mapa de cores indicando as tensões segundo o critério de resistência de von Mises (em N/mm²).

Figura 4.70 - (a) solução do problema de otimização topológica com estimativa inicial de 0,85; (b) mapa de cores indicando as tensões segundo o critério de resistência de von Mises (em N/mm²).

Figura 4.71 - Configuração final e completa da estrutura correspondente à Figura 4.66... 142

Figura 4.72 - Configuração final e completa da estrutura correspondente à Figura 4.68... 142

Figura 4.73 - Configuração final e completa da estrutura correspondente à Figura 4.69... 142

Figura 4.74 - Configuração final e completa da estrutura correspondente à Figura 4.70...

Figura 4.75 - (a) $1^{\circ}$ caso de carregamento do problema de OT; (b) $2^{\circ}$ caso de carregamento do problema de OT

Figura 4.76 - Topologia extraída do artigo publicado por Nishiwaki et al. (2001).

Figura 4.77 - Topologia obtida através da maximização da energia de deformação

Figura 4.78 - Configuração deformada correspondente ao $1^{\circ}$ caso de carregamento..........

Figura 4.79 - Configuração deformada correspondente ao $2^{\circ}$ caso de carregamento..........

Figura 4.80 - (a) $1^{\circ}$ caso de carregamento do problema de OT; (b) $2^{\circ}$ caso de carregamento do problema de OT. 
Figura 4.81 - Topologia obtida para o problema de multi-flexibilidade........................... 148

Figura 4.82 - Configuração deformada correspondente ao $1^{\circ}$ caso de carregamento......... 148

Figura 4.83 - Configuração deformada correspondente ao $2^{\circ}$ caso de carregamento......... 148

Figura A.1 - Estrutura bidimensional de espessura constante igual a $1 \mathrm{~mm}$.................... 160

Figura A.2 - Configuração deformada do segmento $\overline{\mathrm{AO}}$............................................... 161 


\section{LISTA DE TABELAS}

Tabela 4.1 - Comparação entre os deslocamentos observados nas diferentes etapas de projeto.

Tabela 4.2 - Comparação entre os deslocamentos observados nas diferentes etapas de projeto

Tabela 4.3 - Comparação entre os deslocamentos observados nas diferentes etapas de projeto.

Tabela 4.4 - Comparação entre os valores nas diferentes etapas de projeto com restrição volumétrica de $15 \%$.

Tabela 4.5 - Comparação entre os valores nas diferentes etapas de projeto com restrição volumétrica de $20 \%$.

Tabela 4.6 - Comparação entre os valores nas diferentes etapas de projeto com restrição volumétrica de $25 \%$.

Tabela 4.7 - Deslocamentos nas duas etapas de projeto, sendo a OT feita através do código de Sigmund (2001a).

Tabela 4.8 - Deslocamentos nas duas etapas de projeto representadas pelas Figuras 4.68a e $4.68 b$.

Tabela 4.9 - Deslocamentos nas duas etapas de projeto representadas pelas Figuras 4.69a e 4.69b.

Tabela 4.10 - Deslocamentos nas duas etapas de projeto representadas pelas Figuras 4.70a e 4.70b.

Tabela 4.11 - Comparação entre os deslocamentos observados nas diferentes etapas de projeto.

Tabela 4.12 - Comparação entre os deslocamentos observados nas diferentes etapas de projeto

Tabela A.1 - Comparação entre os deslocamentos verticais obtidos a partir do ANSYS 10.0 e da rotina de MEF. 


\section{LISTA DE ABREVIATURAS E SIGLAS}

\begin{tabular}{|c|c|}
\hline ANSYS & Software comercial de elementos finitos \\
\hline DFE & Domínio Fixo Estendido \\
\hline $\mathrm{EF}$ & Elementos Finitos \\
\hline EM & Eficiência mecânica \\
\hline FSD & Free Software Directory \\
\hline FSF & Free Software Foundation \\
\hline $\mathrm{GHz}$ & Gigahertz \\
\hline GLPK & Rotina de programação linear \\
\hline GPa & Gigapascal \\
\hline Internet & Rede mundial de computadores \\
\hline Função-KS & Função Kreisselmeier - Steinhauser \\
\hline MB & Megabyte \\
\hline MEF & Método dos Elementos Finitos \\
\hline MEMS & Microeletromechanical Systems \\
\hline MMA & Method of Moving Asymptotes \\
\hline MOLE & Monotonicity Based Minimum Length Scale \\
\hline MOT & Método da Otimização Topológica \\
\hline OT & Otimização Topológica \\
\hline PL & Programação Linear \\
\hline PLS & Programação Linear Seqüencial \\
\hline RAM & Random Access Memory \\
\hline SIMP & Simple Isotropic Material with Penalization \\
\hline SIMPLEX & Método de programação linear \\
\hline Unesco & United Nations Education, Scientific and Cultural Organization \\
\hline VG & Vantagem geométrica \\
\hline VM & Vantagem mecânica \\
\hline
\end{tabular}




\section{LISTA DE SÍMBOLOS}

$\mathbf{u}_{\mathrm{e}}$

$\mathrm{U}_{\mathrm{e}}$

$\boldsymbol{\varepsilon}_{\mathrm{e}}$

$\boldsymbol{\sigma}_{\mathrm{e}}$

$\mathrm{C}_{\mathrm{e}}$

E

V

B

$\mathbf{k}_{\mathrm{e}}$

$\mathbf{f}_{\mathrm{e}}$

$\mathrm{v}_{\mathrm{e}}$

$t_{e}$

$\mathrm{A}_{\mathrm{e}}$

$\mathbf{J}$

$\mathbf{k}_{\mathrm{g}}$

$\mathbf{u}_{\mathrm{g}}$

$\mathbf{f}_{\mathrm{g}}$

$\mathbf{x}$

$\rho(\mathbf{x})$

$\mathbf{E}(\mathbf{x})$

Abscissa do sistema de coordenadas locais do elemento finito

Ordenada do sistema de coordenadas locais do elemento finito

Abscissa do sistema de coordenadas globais

Ordenada do sistema de coordenadas globais

Componente horizontal do deslocamento de um ponto do elemento finito

Componente vertical do deslocamento de um ponto do elemento finito

Matriz das funções de forma do elemento finito

Vetor dos deslocamentos nodais do elemento finito

Energia de deformação do elemento finito

Vetor das deformações pontuais do elemento finito

Vetor das tensões pontuais do elemento finito

Matriz de elasticidade do elemento finito

Módulo de elasticidade longitudinal

Coeficiente de Poisson

Matriz dos operadores diferenciais aplicados sobre as funções de forma

Matriz de rigidez do elemento finito

Vetor das forças nodais do elemento finito

Volume do elemento finito

Espessura do elemento finito

Área do elemento finito

Matriz Jacobiana

Matriz de rigidez do domínio fixo de projeto

Vetor dos deslocamentos nodais do domínio fixo de projeto

Vetor das forças nodais aplicadas no domínio fixo de projeto

Vetor posição

Densidade pontual

Tensor constitutivo de material 


\begin{tabular}{|c|c|}
\hline $\mathbf{E}^{0}$ & Tensor constitutivo do material base \\
\hline$\theta$ & Ângulo de rotação da microestrutura \\
\hline$a$ & Dimensão interna da microestrutura \\
\hline$b$ & Dimensão interna da microestrutura \\
\hline $\mathrm{n}$ & Coeficiente de penalização \\
\hline$\Omega$ & Domínio fixo de projeto \\
\hline$\Omega^{\mathrm{mat}}$ & Região do domínio fixo de projeto com material base \\
\hline $\mathrm{E}^{0}$ & Módulo de elasticidade longitudinal do material base \\
\hline $\mathbf{t}$ & Tensor de carregamento \\
\hline $\mathbf{u}$ & Tensor de primeira ordem representando os deslocamentos pontuais \\
\hline$\Gamma_{\mathrm{t}}$ & Superfície de aplicação do carregamento \\
\hline$\Gamma_{\mathrm{d}}$ & Superfície engastada \\
\hline $\mathrm{L}^{\mathrm{i}}\left(\mathrm{u}^{\mathrm{j}}\right)$ & Energia mútua \\
\hline $\mathrm{t}_{\mathrm{i}}$ & Diagonal para teste de "monotonicidade" \\
\hline $\mathcal{M}_{\mathrm{d}, \mathrm{t}_{\mathrm{i}}}$ & Funcional para teste de "monotonicidade" \\
\hline $\mathcal{L}$ & Restrição global para teste de "monotonicidade" \\
\hline $\mathrm{m}$ & Teste de "monotonicidade" simplificado \\
\hline $\mathrm{h}$ & Restrição global para teste de "monotonicidade" simplificado \\
\hline$\varepsilon$ & Tensor das deformações pontuais \\
\hline$\sigma$ & Tensor das tensões pontuais \\
\hline $\mathrm{W}$ & Energia de deformação específica \\
\hline $\mathrm{w}^{*}$ & Energia de deformação específica penalizada \\
\hline $\mathrm{U}$ & Energia de deformação do domínio fixo de projeto \\
\hline$\phi$ & Função objetivo do problema contínuo \\
\hline $\mathrm{V}_{\text {total }}$ & Volume máximo de material \\
\hline $\mathrm{c}$ & Número de carregamentos \\
\hline$\phi_{\mathrm{MF}}$ & Função multi-objetivo do problema contínuo \\
\hline $\mathrm{w}_{\mathrm{e}}$ & Energia de deformação específica para os pontos do elemento finito \\
\hline$\rho_{\mathrm{e}}$ & Densidade do elemento finito \\
\hline $\mathrm{N}$ & Número de elementos finitos \\
\hline$\Phi$ & Função objetivo do problema numérico \\
\hline
\end{tabular}




\begin{tabular}{|c|c|}
\hline $\mathbf{f}_{\mathrm{a}}$ & Força pontual aplicada no ponto A \\
\hline $\mathrm{k}_{\mathrm{b}}$ & Constante de rigidez da mola acoplada ao ponto B \\
\hline$\Phi_{\mathrm{MF}}$ & Função multi-objetivo do problema numérico \\
\hline $\mathbf{H}_{\mathrm{e}}$ & Operador matricial que localiza os deslocamentos nodais do elemento finito \\
\hline$\lambda_{\mathrm{g}}$ & Vetor solução do primeiro problema adjunto \\
\hline $\mathbf{L}_{\mathrm{j}}$ & Operador vetorial que localiza o deslocamento restringido $\mathrm{j}$ \\
\hline$\gamma_{\mathrm{g}}$ & Vetor solução do segundo problema adjunto \\
\hline$\Phi_{\mathrm{L}}$ & Função objetivo linearizada \\
\hline$\rho$ & Vetor das variáveis de projeto \\
\hline$F(x)$ & Função não-linear \\
\hline$f(x)$ & Função linear \\
\hline$x_{\min }$ & Limite móvel inferior da variável de projeto \\
\hline$x_{\max }$ & Limite móvel superior da variável de projeto \\
\hline$x^{\mathrm{k}}$ & Variável de projeto correspondente à iteração $\mathrm{k}$ \\
\hline$\Phi_{\mathrm{MF}_{\mathrm{L}}}$ & Função multi-objetivo linearizada \\
\hline$\rho_{\mathrm{i}}^{\text {ajustado }}$ & Densidade do elemento i ajustada pelo filtro de densidades \\
\hline$w$ & Peso de filtragem \\
\hline $\bar{w}$ & Média ponderada dos pesos de filtragem \\
\hline nv & Número de elementos finitos localizados no campo do raio de abrangência \\
\hline $\mathrm{R}_{\max }$ & Raio de abrangência do filtro espacial de densidades \\
\hline $\mathrm{R}_{\mathrm{ij}}$ & Distância entre os centróides dos elementos finitos \\
\hline
\end{tabular}




\section{SUMÁRIO}

1 INTRODUÇÃ

1.1 CONCEITOS INICIAIS ................................................................................. 21

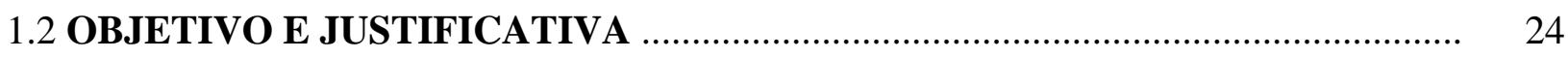

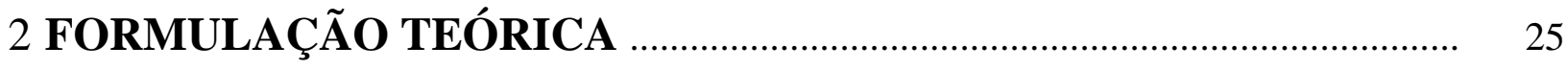

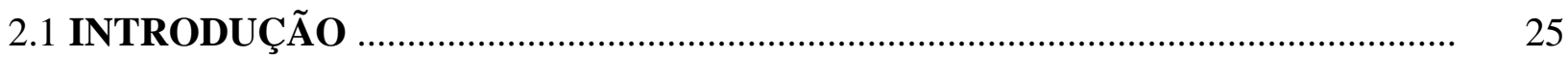

2.2 MÉTODO DOS ELEMENTOS FINITOS ............................................................ 26

2.2.1 Comentários iniciais ....................................................................................... 26

2.2.2 Formulação do elemento finito isoparamétrico de quatro nós ................................. 26

2.2.3 Determinação dos deslocamentos nodais da estrutura ............................................. 32

2.3 MÉTODO DA OTIMIZAÇÃO TOPOLÓGICA …………………………………..... 33

2.3.1 Conceitos iniciais ........................................................................................................ 33

2.3.2 Histórico ……............................................................................................... 35

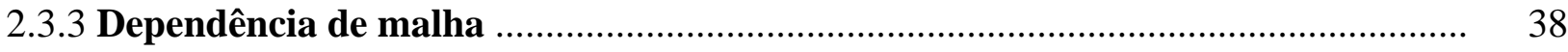

2.3.4 Modelos de materiais ........................................................................................... 40

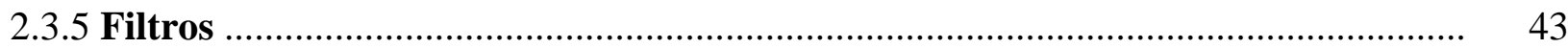

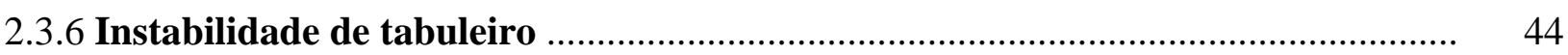

2.4 OTIMIZAÇÃO TOPOLÓGICA NO PROJETO DE MECANISMOS

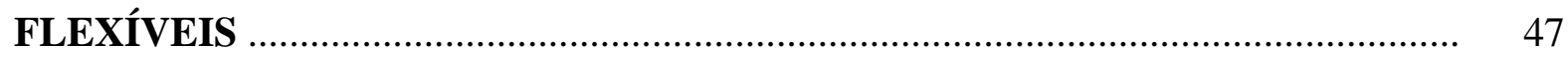

2.5 EFEITO “DOBRADIÇA” .................................................................................. 52

2.6 FORMULAÇÃO DO PROBLEMA DE OTIMIZAÇÃO TOPOLÓGICA ............... 58

2.6.1 Comentários iniciais ........................................................................................... 58

2.6.2 Formulação contínua do problema de otimização ………………………………... 58

2.6.3 Formulação contínua do problema de otimização aplicado à multi-flexibilidade . 63

2.6.4 Formulação numérica do problema de otimização ……………………………....... 66

2.6.5 Formulação numérica do problema de otimização aplicado à multi-flexibilidade 69

2.7 ANÁLISE DAS SENSIBILIDADES ...................................................................... 71

2.7.1 Comentários iniciais ................................................................................................ 71

2.7.2 Sensibilidades da função objetivo ………………...............................................

2.7.3 Sensibilidades dos deslocamentos restringidos ………………………………........ 


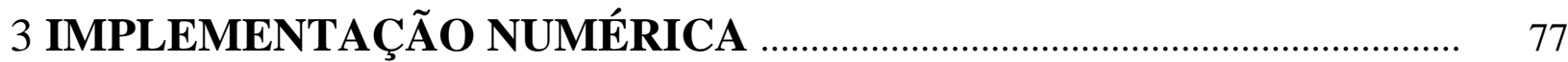

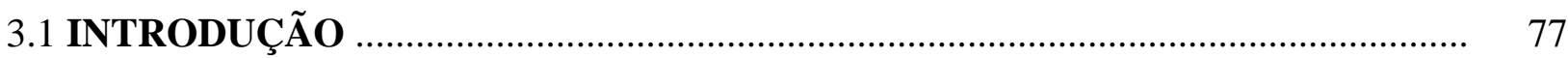

3.2 PROGRAMAÇÃO LINEAR SEQÜENCIAL (PLS) ……………………………..... 79

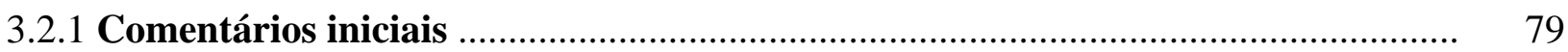

3.2.2 Linearização das funções objetivo e multi-objetivo …………………………......... 80

3.2.3 Linearização das funções que representam os deslocamentos restringidos ........... 83

3.2.4 Formulação do subproblema linear ....................................................................... 86

3.3 IMPLEMENTAÇÃO DO FILTRO ESPACIAL DE DENSIDADES ......................... 88

3.4 ESTRUTURA DO CÓDIGO COMPUTACIONAL ................................................ 91

4 RESULTADOS E DISCUSSÕES....................................................................... 94

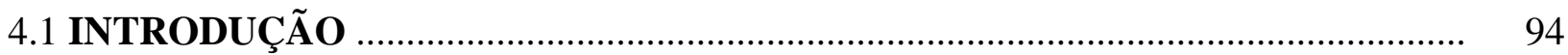

4.2 RESULTADOS PRELIMINARES ................................................................... 95

4.2.1 Sensibilidade em relação aos valores iniciais atribuídos às variáveis de projeto .. 95

4.2.2 Sensibilidade em relação ao refinamento da malha do domínio fixo ………........... 98

4.2.3 Ocorrência da instabilidade de tabuleiro ………………………………………..... 100

4.3 RESULTADOS OBTIDOS COM A ATIVAÇÃO DO FILTRO …………………..... 102

4.3.1 Comentários iniciais ........................................................................................... 102

4.3.2 Problema não-convexo ……………………………………………………….. 102

4.3.3 Independência de malha …………………………………………………….... 104

4.3.4 Redução da instabilidade de tabuleiro …………………………......................... 107

4.4 ASPECTOS CARACTERÍSTICOS DAS TOPOLOGIAS ...................................... 112

4.4.1 Comentários iniciais ......................................................................................... 112

4.4.2 Redução da ocorrência de “dobradiças” ................................................................... 113

4.4.3 Comportamento cinemático do mecanismo flexível ................................................ 117

4.5 AVALIAÇÃO DO EFEITO DA VARIAÇÃO DO RAIO DO FILTRO E DO

VOLUME RESTRINGIDO ............................................................................. 120

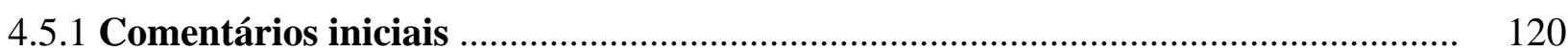

4.5.2 Variação da restrição do volume de material ………………………………......... 121

4.5.3 Redução das escalas de cinza por meio da desativação do filtro ….......................... 124 
4.5.4 Refinamento da malha e redução do raio de abrangência do esquema de filtragem

4.6 AVALIAÇÃO DO EFEITO DA VARIAÇÃO DA RIGIDEZ E DO DESLOCAMENTO DA MOLA

4.6.1 Resultado inicial para referência …...................................................................... 132

4.6.2 Redução da restrição imposta ao deslocamento de saída ........................................ 133

4.6.3 Redução da rigidez da mola .............................................................................. 134

4.6.4 Supressão da mola e da restrição de saída ............................................................ 135

4.7 PROJETO DE ESTRUTURAS RÍGIDAS ......................................................... 136

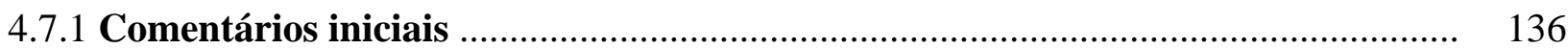

4.7.2 Variação do coeficiente de penalização das densidades ....................................... 137

4.7.3 Variação da estimativa inicial de otimização ......................................................... 139

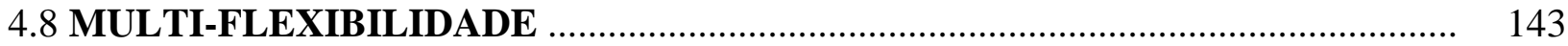

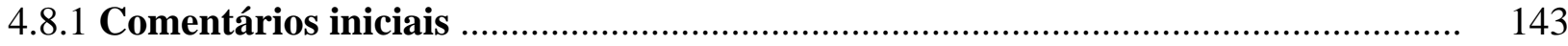

4.8.2 Redução da ocorrência de “dobradiças” para problemas de multi-flexibilidade .. 143

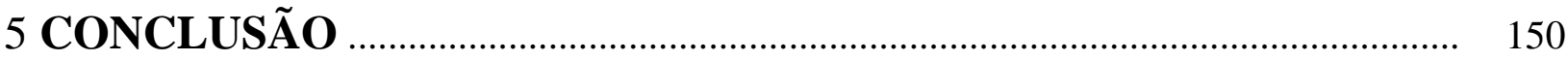

5.1 CONCLUSÕES E OBSERVAÇÕES FINAIS ….................................................. 150

5.2 SUGESTÕES PARA FUTUROS TRABALHOS …........................................... 151

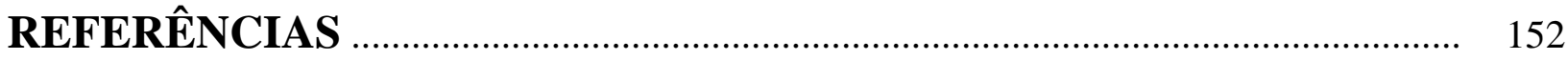

APÊNDICE A - Aferição da rotina de elementos finitos …............................ 160 


\section{INTRODUÇÃO}

\subsection{CONCEITOS INICIAIS}

Os mecanismos flexíveis são dispositivos que exploram a flexibilidade estrutural para transmitir força e movimento a outros componentes mecânicos. Assim, diferem dos mecanismos convencionais constituídos por corpos rígidos, cuja deformação elástica é indesejável devido à alteração que impõe ao comportamento cinemático inicialmente previsto para o projeto. $\mathrm{Na}$ atualidade, é possível encontrar, nas mais diversas áreas, inúmeros produtos industrializados providos de mecanismos flexíveis em seus sistemas funcionais. A título exemplificativo, têm-se os sistemas microeletromecânicos - microeletromechanical systems (MEMS) -, que são integráveis a circuitos eletrônicos (PETERSEN, 1982). Estes sistemas são fabricados em escala micrométrica, sendo que, entre vários outros exemplos, pode-se citar os seguintes equipamentos nos quais os MEMS são encontrados: sensores de aceleração para airbags (acessórios de segurança veicular), instrumentos cirúrgicos intracorpóreos, aparelhos de auxílio para a audição, micromanipuladores e equipamentos utilizados em nanofabricação (KIM; PISANO; MULLER, 1992). Mecanismos flexíveis são bem adequados em dispositivos de mecânica de precisão, como no caso dos sistemas posicionadores dos espelhos empregados em equipamentos ópticos. Os sistemas automotivos também oferecem um campo de aplicação bastante amplo. Como exemplo, um "limpador de pára-brisas” constituído de uma única estrutura flexível é demonstrado por Kota et al. (2001).

Os mecanismos flexíveis, conhecidos na literatura internacional como compliant mechanisms, apresentam menos desgaste e menos atrito, pois são formados por um único corpo, dispensando montagens e lubrificações (HOWELL; MIDHA, 1994; SIGMUND, 1997). Por estas razões, são bem adequados aos MEMS, cujas dimensões micrométricas demandam tais vantagens. Ainda com respeito às aplicações em MEMS, os mecanismos flexíveis podem ser fabricados pelas mesmas técnicas empregadas na indústria dos semicondutores, ou seja, ataques químicos sobre a superfície de um material preparado adequadamente (substrato). Com a utilização destas técnicas é possível obter qualquer contorno para uma estrutura plana, sendo este o aspecto mais comum de um mecanismo flexível utilizado em sistemas cujas dimensões sejam micrométricas. Entre as desvantagens, o 
problema da fadiga pode ser considerado como um dos mais críticos, tendo sido objeto de discussão e estudo em algumas publicações (HOWELL, 2001; BAHIA, 2005).

Existem duas abordagens possíveis para o projeto sistêmico dos mecanismos flexíveis (FRECKER; KIKUCHI; KOTA, 1996). Na primeira, conhecida como síntese cinemática (MIDHA; NORTON; HOWELL, 1992), toma-se por base o conhecimento tradicional da cinemática dos corpos rígidos. Assim, a partir de uma cadeia cinemática definida por corpos rígidos unidos por juntas articuláveis, Her e Midha (1987) obtiveram mecanismos cuja flexibilidade se concentrava em pivôs flexíveis localizados em determinados pontos da estrutura.

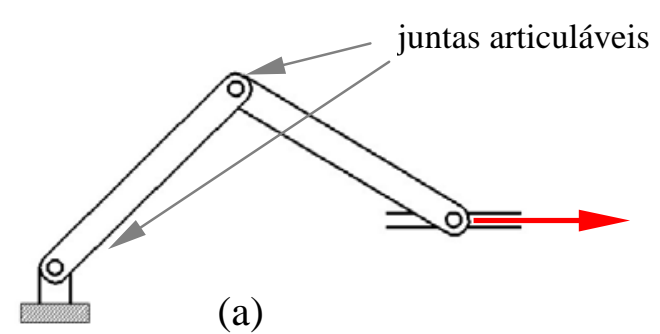

(a)

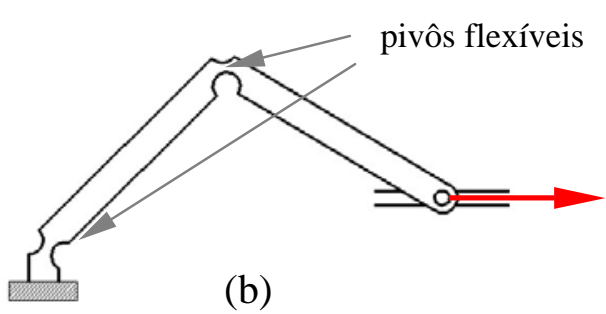

(b)

Figura 1.1 - (a) mecanismo formado por corpos rígidos; (b) mecanismo equivalente com os pivôs flexíveis.

Como uma evolução desta primeira abordagem, Howell e Midha (1994, 1996) sugeriram um método pelo qual o mecanismo flexível era obtido a partir de um mecanismo inicial cujos movimentos se davam em função de molas torcionais posicionadas nas juntas articuláveis utilizadas para unir os componentes rígidos (mecanismo de pseudo-corpo rígido). O método se mostrou bem adequado para lidar com grandes deslocamentos.

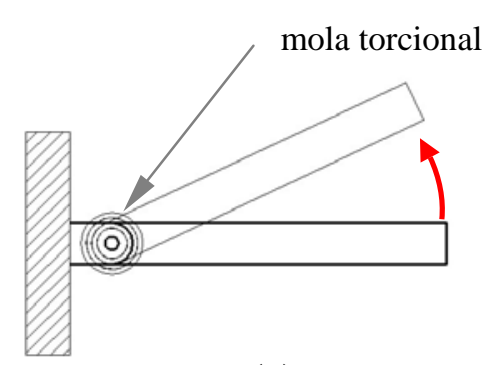

(a)

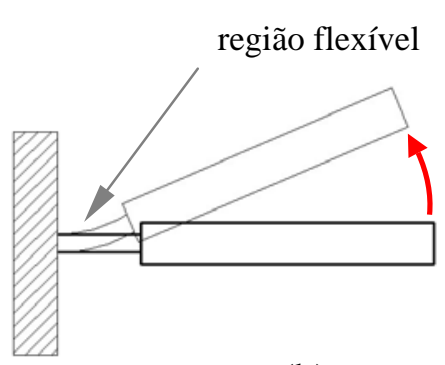

(b)

Figura 1.2 - (a) mecanismo de pseudo-corpo rígido; (b) mecanismo flexível equivalente. 
Howell (2001) apresenta as relações de equivalência cinemática entre os pseudocorpos rígidos e as várias configurações geométricas com as quais certos componentes flexíveis podem se apresentar. Deste modo, torna-se possível projetar, através da combinação destes componentes flexíveis, um mecanismo flexível equivalente a uma cadeia constituída por pseudo-corpos rígidos.

A segunda abordagem é caracterizada pela síntese dos mecanismos flexíveis a partir de meios contínuos (ANANTHASURESH; KOTA; GIANCHANDANI, 1994). Assim, através do método da otimização topológica (BENDSØE; KIKUCHI, 1988), distribui-se uma quantidade limitada de material fazendo com que um dispositivo possa ser sintetizado num espaço fechado denominado domínio de projeto. O objetivo é obter a topologia de um mecanismo cuja flexibilidade esteja distribuída em seu interior, sempre de modo a transmitir força e movimento de forma otimizada. No exemplo da Figura 1.3d, com a aplicação da força no ponto A, o mecanismo flexível obtido deforma a mola conectada ao ponto B.

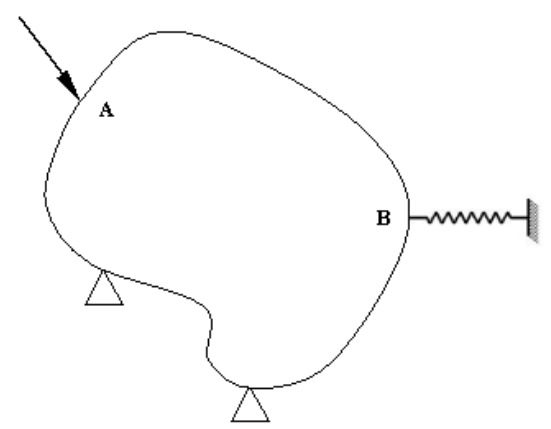

(a)

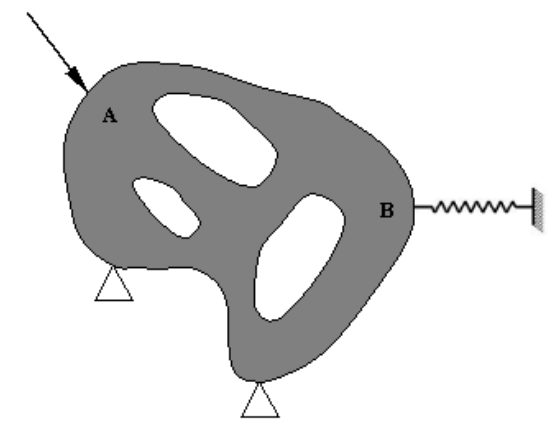

(c)

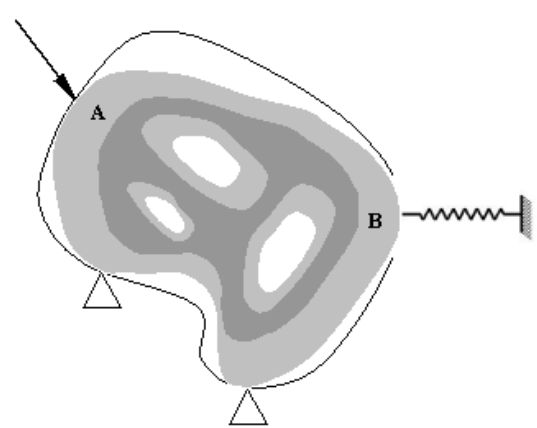

(b)

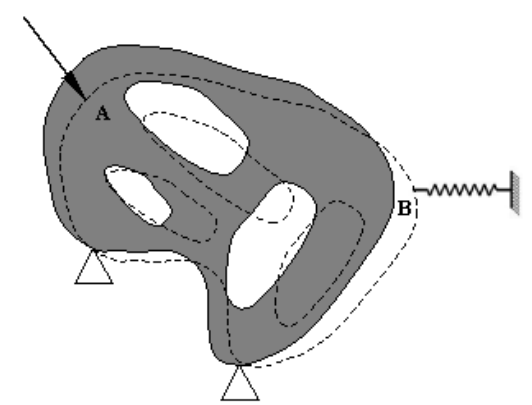

(d)

Figura 1.3 - (a) domínio de projeto; (b) quantidade limitada de material sendo distribuida no domínio de projeto; (c) resultado da otimização topológica; (d) configuração deformada do mecanismo sintetizado. 


\subsection{OBJETIVO E JUSTIFICATIVA}

Conforme dito na seção anterior, as possibilidades de aplicação para os mecanismos flexíveis são muito amplas, por isso, na literatura especializada, encontram-se várias formulações matemáticas que fazem uso do método da otimização topológica para projetá-los. Cada uma delas privilegia uma característica em detrimento a outras. Assim, pode-se, por exemplo, utilizar uma certa formulação para projetar mecanismos flexíveis que transmitam forças mais intensas por meio de deslocamentos menores ou vice-versa. Neste trabalho, algumas das formulações mais conhecidas serão apresentadas e discutidas. Embora tenham objetivos e aplicações diferentes, um aspecto comum a todas é a ocorrência de uma instabilidade numérica pela qual a conectividade de duas regiões sólidas do mecanismo flexível obtido se dá através de um único nó pertencente à malha de elementos finitos ${ }^{1}$. Na tarefa de transmitir movimento, este nó faz as vezes de uma articulação ou “dobradiça”, o que na prática confere ao dispositivo projetado características de mecanismo de corpo rígido. A presença destas “dobradiças” na solução final de projeto é altamente indesejável, pois elas dificultam a fabricação e, principalmente, comprometem a modelagem do mecanismo flexível. Existem muitos trabalhos que abordam o assunto e oferecem formas de contornar o problema. Assim, algumas das publicações relacionadas foram pesquisadas e mencionadas neste texto, o que dá subsídios à utilização de uma formulação cuja implementação em um código computacional atenda o objetivo de projetar mecanismos flexíveis menos suscetíveis à ocorrência das “dobradiças”. Adicionalmente, investiga-se a aplicação deste código ao projeto de estruturas rígidas. Outro objetivo deste trabalho é estender a formulação ao projeto de mecanismos multi-flexíveis (mecanismos que apresentam diferentes formas de deformação em função do carregamento aplicado).

A otimização topológica vem se estabelecendo como uma ferramenta muito importante no auxílio ao projeto de componentes mecânicos. Muito se avançou desde os seus trabalhos iniciais, onde algumas inadequações foram observadas e solucionadas pelas últimas pesquisas. Contudo, ainda há margem para discussão em relação à algumas questões, como no caso das “dobradiças”, comuns nos mecanismos flexíveis. Como estes são dispositivos de uso cada vez mais freqüente, justifica-se a necessidade de projetá-los sem que o problema ocorra.

\footnotetext{
${ }^{1} \mathrm{O}$ método dos elementos finitos é empregado na maioria dos trabalhos que utilizam a otimização topológica como forma sistemática para projetar componentes mecânicos. No capítulo 2 serão demonstrados mais detalhes com respeito à sua aplicação.
} 


\section{FORMULAÇÃO TEÓRICA}

\subsection{INTRODUÇÃO}

Este capítulo aborda a fundamentação teórica necessária à elaboração de um procedimento computacional capaz de projetar mecanismos flexíveis bidimensionais menos suscetíveis aos problemas decorrentes da aplicação do método da otimização topológica (MOT) (BENDSØE; SOARES, 1992; BENDSØE; SIGMUND, 2003), como a dependência do resultado final em relação ao nível de discretização da malha de elementos finitos (EF), a presença das escalas de cinza, a ocorrência da instabilidade de tabuleiro e, principalmente, o surgimento das “dobradiças”.

Essencialmente, o MOT implementado neste trabalho combina o método dos elementos finitos (BATHE, 1996) com uma rotina de otimização baseada no método da programação linear seqüencial (HAFTKA; GÜRDAL; KAMAT, 1990). Portanto, uma breve revisão teórica sobre o método dos elementos finitos é apresentada na seção 2.2. Quanto à programação linear seqüencial, mais detalhes serão demonstrados no capítulo 3. A seção 2.3 aborda os aspectos relacionados ao MOT, dentre os quais as suas principais aplicações e os seus problemas mais comuns. A utilização do MOT no projeto de mecanismos flexíveis e a discussão que expõe a questão do efeito “dobradiça” são os assuntos das seções 2.4 e 2.5, respectivamente. Na seção 2.6, expõem-se os conceitos físicos que fundamentam a formulação matemática do problema de otimização topológica aplicado neste trabalho. Para encontrar a solução deste problema (obtenção da topologia “ótima”), faz-se necessário calcular os gradientes das funções envolvidas (função objetivo e demais restrições). Assim, na seção 2.7, deduzem-se as equações com as quais os valores numéricos destes gradientes são determinados (análise das sensibilidades). 


\subsection{MÉTODO DOS ELEMENTOS FINITOS}

\subsubsection{Comentários iniciais}

O método dos elementos finitos (MEF) ganhou força com o aumento da capacidade de processamento oferecida pelos computadores ao longo dos últimos anos, deste modo, desde a sua popularização na década de 70, o MEF tem se difundido em várias áreas da Engenharia. Neste trabalho, conforme será visto adiante, a cada iteração do código computacional utilizado para o projeto de mecanismos flexíveis, o método é aplicado na análise estática estrutural efetuada sobre o domínio fixo ${ }^{1}$ do problema de otimização. Sabe-se, da teoria do MEF (BATHE, 1996), que as características elásticas de uma estrutura sólida são representadas por sua matriz de rigidez, que é comumente chamada de matriz de rigidez global, sendo que esta é obtida através da composição das várias matrizes de rigidez cada uma das quais correspondente a um elemento finito presente na malha da referida estrutura. Uma breve revisão teórica será mostrada a seguir apenas com o objetivo de reforçar a teoria envolvida com o problema abordado neste trabalho. Assim, na seção 2.2.2, têm-se as considerações acerca do elemento finito isoparamétrico de quatro nós. O cálculo dos deslocamentos nodais da estrutura é demonstrado na seção 2.2.3.

\subsubsection{Formulação do elemento finito isoparamétrico de quatro nós}

Este tipo de elemento finito é representado num plano, sendo, portanto, bidimensional. Cada um de seus quatro nós possui dois graus de liberdade locais, conforme pode ser visto na Figura 2.1.

\footnotetext{
${ }^{1} \mathrm{O}$ domínio fixo do problema de otimização corresponde à região do espaço a partir da qual o mecanismo flexível será projetado. Este conceito será discutido na seção 2.3.
} 


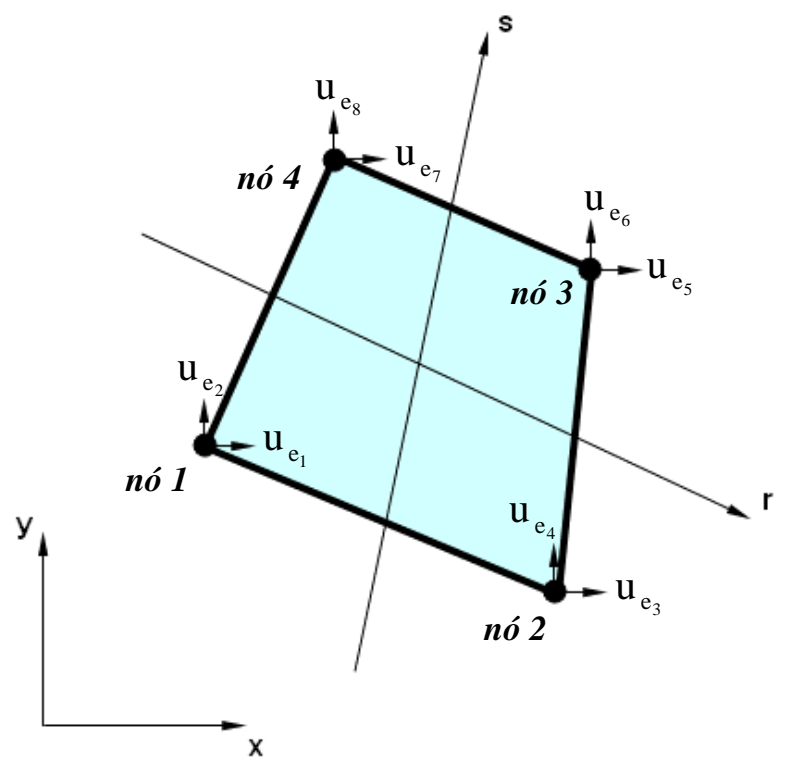

Figura 2.1 - Elemento isoparamétrico de quatro nós.

As coordenadas globais - em relação ao sistema (x, y) - de um ponto interno qualquer do elemento finito são expressas através das funções de forma e das coordenadas globais dos nós. Neste trabalho, estas funções de forma, ditas bilineares, são as seguintes:

$$
\begin{aligned}
& \mathrm{N}_{1}=\frac{1}{4}(1-\mathrm{r})(1-\mathrm{s}) \quad \text { com }-1 \leq \mathrm{r} \leq 1 \quad \text { e }-1 \leq \mathrm{s} \leq 1 \\
& \mathrm{~N}_{2}=\frac{1}{4}(1+\mathrm{r})(1-\mathrm{s}) \quad \text { com }-1 \leq \mathrm{r} \leq 1 \quad \text { e }-1 \leq \mathrm{s} \leq 1 \\
& \mathrm{~N}_{3}=\frac{1}{4}(1+\mathrm{r})(1+\mathrm{s}) \quad \text { com }-1 \leq \mathrm{r} \leq 1 \quad \text { e }-1 \leq \mathrm{s} \leq 1 \\
& \mathrm{~N}_{4}=\frac{1}{4}(1-\mathrm{r})(1+\mathrm{s}) \quad \text { com }-1 \leq \mathrm{r} \leq 1 \quad \text { e }-1 \leq \mathrm{s} \leq 1
\end{aligned}
$$

onde os eixos $\mathrm{r}$ e s representam o sistema de coordenadas locais do elemento. Logo, em relação às coordenadas globais do referido ponto interno, pode-se escrever as equações a seguir. 


$$
\begin{aligned}
& \mathrm{x}=\mathrm{N}_{1} \mathrm{x}_{1}+\mathrm{N}_{2} \mathrm{x}_{2}+\mathrm{N}_{3} \mathrm{x}_{3}+\mathrm{N}_{4} \mathrm{x}_{4} \\
& \mathrm{y}=\mathrm{N}_{1} \mathrm{y}_{1}+\mathrm{N}_{2} \mathrm{y}_{2}+\mathrm{N}_{3} \mathrm{y}_{3}+\mathrm{N}_{4} \mathrm{y}_{4}
\end{aligned}
$$

Da mesma maneira, é possível escrever as componentes do deslocamento deste mesmo ponto interno do elemento através das funções de forma e das componentes dos deslocamentos nodais com respeito ao sistema (x, y), conforme mostram as equações (2.7) e (2.8).

$$
\begin{aligned}
& \mathrm{u}_{\mathrm{e}_{\mathrm{x}}}(\mathrm{r}, \mathrm{s})=\mathrm{N}_{1} \mathrm{u}_{\mathrm{e}_{1}}+\mathrm{N}_{2} \mathrm{u}_{\mathrm{e}_{3}}+\mathrm{N}_{3} \mathrm{u}_{\mathrm{e}_{5}}+\mathrm{N}_{4} \mathrm{u}_{\mathrm{e}_{7}} \\
& \mathrm{u}_{\mathrm{e}_{\mathrm{y}}}(\mathrm{r}, \mathrm{s})=\mathrm{N}_{1} \mathrm{u}_{\mathrm{e}_{2}}+\mathrm{N}_{2} \mathrm{u}_{\mathrm{e}_{4}}+\mathrm{N}_{3} \mathrm{u}_{\mathrm{e}_{6}}+\mathrm{N}_{4} \mathrm{u}_{\mathrm{e}_{8}}
\end{aligned}
$$

Assim, nas equações acima, $u_{e_{x}}(r, s)$ e $u_{e_{y}}(r, s)$ são as componentes - nas direções dos eixos x e y, respectivamente - correspondentes ao deslocamento do ponto (r, s) referenciado em relação às coordenadas locais - pertencente ao interior do elemento finito. As equações (2.7) e (2.8) podem ser representadas pela seguinte equação matricial.

$$
\begin{aligned}
& \left\{\begin{array}{l}
\mathrm{u}_{\mathrm{e}_{\mathrm{x}}}(\mathrm{r}, \mathrm{s}) \\
\mathrm{u}_{\mathrm{e}_{\mathrm{y}}}(\mathrm{r}, \mathrm{s})
\end{array}\right\}=\left[\begin{array}{cccccccc}
\mathrm{N}_{1} & 0 & \mathrm{~N}_{2} & 0 & \mathrm{~N}_{3} & 0 & \mathrm{~N}_{4} & 0 \\
0 & \mathrm{~N}_{1} & 0 & \mathrm{~N}_{2} & 0 & \mathrm{~N}_{3} & 0 & \mathrm{~N}_{4}
\end{array}\right]\left\{\begin{array}{l}
\mathrm{u}_{\mathrm{e}_{1}} \\
\mathrm{u}_{\mathrm{e}_{2}} \\
\mathrm{u}_{\mathrm{e}_{3}} \\
\mathrm{u}_{\mathrm{e}_{4}} \\
\mathrm{u}_{\mathrm{e}_{5}} \\
\mathrm{u}_{\mathrm{e}_{6}} \\
\mathrm{u}_{\mathrm{e}_{7}} \\
\mathrm{u}_{\mathrm{e}_{8}}
\end{array}\right\} \\
& \mathbf{u}_{\mathrm{e}_{\mathrm{x}, \mathrm{y}}}(\mathrm{r}, \mathrm{s})=\mathbf{N} \mathbf{u}_{\mathrm{e}}
\end{aligned}
$$

onde $\mathbf{N}$ é a matriz das funções de forma do elemento e $\mathbf{u}_{\mathrm{e}}$ é o vetor das componentes dos deslocamentos nodais, cada uma das quais associada a um grau de liberdade local do elemento finito. 
A matriz de rigidez do elemento finito pode ser obtida a partir da equação que expressa a sua energia de deformação $\left(U_{e}\right)$, quando este é submetido à aplicação de forças externas em seus nós. Assim, tem-se:

$$
\mathrm{U}_{\mathrm{e}}=\frac{1}{2} \int_{\mathrm{v}_{\mathrm{e}}} \boldsymbol{\varepsilon}_{\mathrm{e}}^{\mathrm{T}}(\mathrm{r}, \mathrm{s}) \boldsymbol{\sigma}_{\mathrm{e}}(\mathrm{r}, \mathrm{s}) \mathrm{dv}_{\mathrm{e}}
$$

onde $\boldsymbol{\varepsilon}_{\mathrm{e}}(\mathrm{r}, \mathrm{s})$ e $\boldsymbol{\sigma}_{\mathrm{e}}(\mathrm{r}, \mathrm{s})$ são, respectivamente, os vetores que definem o estado de deformações e o estado de tensões associados ao ponto (r, s), para o caso de um elemento finito bidimensional. A eq.(2.11), por sua vez, relaciona estas duas grandezas, ou seja:

$$
\boldsymbol{\sigma}_{\mathrm{e}}(\mathrm{r}, \mathrm{s})=\mathbf{C}_{\mathrm{e}} \boldsymbol{\varepsilon}_{\mathrm{e}}(\mathrm{r}, \mathrm{s})
$$

sendo que $\mathbf{C}_{\mathrm{e}}$ é matriz de elasticidade do elemento finito. Esta matriz define as características elásticas do elemento por meio do módulo de elasticidade longitudinal (E) e do coeficiente de Poisson ( $v$ ). Ambos são os parâmetros de material do qual o referido elemento é constituído. Neste trabalho, em que a isotropia dos materiais e o estado plano de tensões foram considerados, a matriz de elasticidade é dada pela eq.(2.12).

$$
\mathbf{C}_{\mathrm{e}}=\frac{\mathrm{E}}{1-v^{2}}\left[\begin{array}{ccc}
1 & v & 0 \\
v & 1 & 0 \\
0 & 0 & \frac{1-v}{2}
\end{array}\right]
$$

Voltando à expressão da energia de deformação, pode-se reescrevê-la substituindo a eq.(2.11) na eq.(2.10). Assim, tem-se:

$$
\mathrm{U}_{\mathrm{e}}=\frac{1}{2} \int_{\mathrm{v}_{\mathrm{e}}} \boldsymbol{\varepsilon}_{\mathrm{e}}^{\mathrm{T}}(\mathrm{r}, \mathrm{s}) \mathbf{C}_{\mathrm{e}} \boldsymbol{\varepsilon}_{\mathrm{e}}(\mathrm{r}, \mathrm{s}) \mathrm{dv} \mathrm{e}_{\mathrm{e}}
$$

o vetor $\boldsymbol{\varepsilon}_{\mathrm{e}}(\mathrm{r}, \mathrm{s})$ é obtido por meio da aplicação dos operadores diferenciais sobre a eq.(2.9), conforme está mostrado a seguir. 


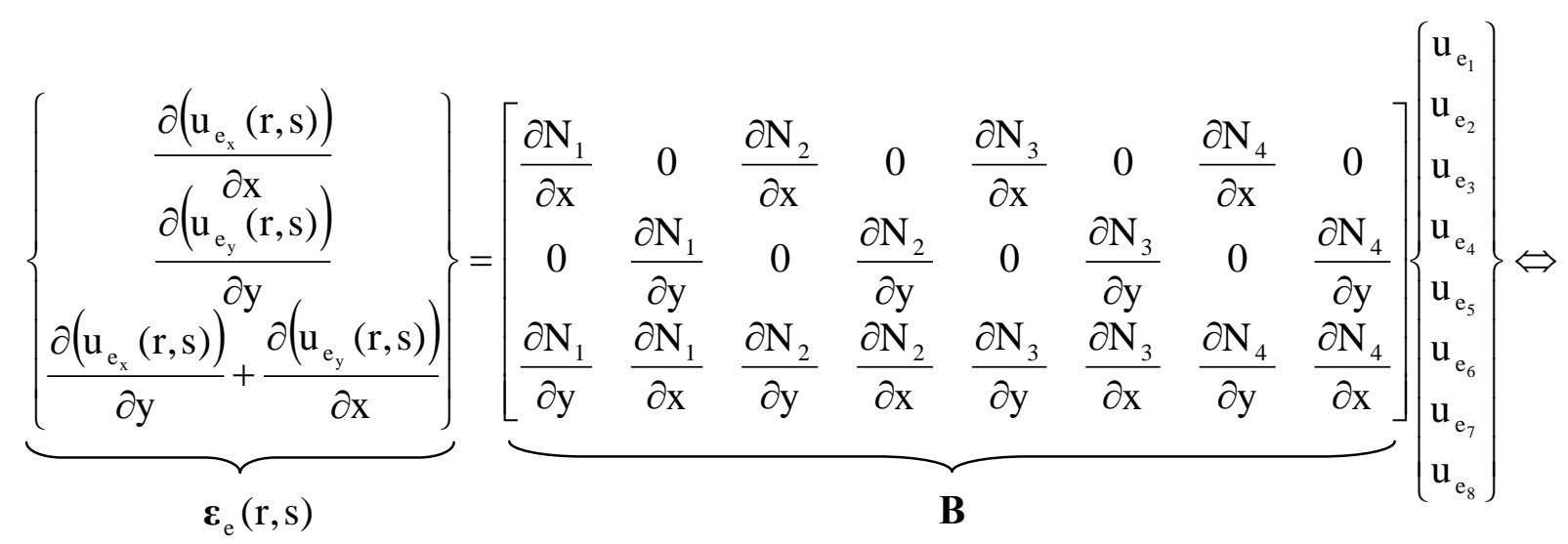

$\boldsymbol{\varepsilon}_{\mathrm{e}}(\mathrm{r}, \mathrm{s})=\mathbf{B} \mathbf{u}_{\mathrm{e}}$

Sabe-se que a matriz de rigidez ( $\mathbf{k}_{\mathrm{e}}$ ) do elemento finito é tal que:

$$
\mathbf{k}_{\mathrm{e}} \mathbf{u}_{\mathrm{e}}=\mathbf{f}_{\mathrm{e}}
$$

onde $\mathbf{f}_{\mathrm{e}}$ é o vetor das componentes das forças - em relação ao sistema (x, y) - aplicadas em cada um dos quatro nós presentes no tipo de elemento discutido neste trabalho. Admitindo-se que o material seja elástico, a energia de deformação também pode ser escrita em função do trabalho das forças externas, ou seja:

$$
\mathrm{U}_{\mathrm{e}}=\frac{1}{2}\left(\mathbf{f}_{\mathrm{e}}^{\mathrm{T}} \mathbf{u}_{\mathrm{e}}\right)
$$

substituindo a eq.(2.15) na eq.(2.16), tem-se:

$$
\mathrm{U}_{\mathrm{e}}=\frac{1}{2}\left[\left(\mathbf{k}_{\mathrm{e}} \mathbf{u}_{\mathrm{e}}\right)^{\mathrm{T}} \mathbf{u}_{\mathrm{e}}\right] \Rightarrow \mathrm{U}_{\mathrm{e}}=\frac{1}{2}\left(\mathbf{u}_{\mathrm{e}}^{\mathrm{T}} \mathbf{k}_{\mathrm{e}} \mathbf{u}_{\mathrm{e}}\right)
$$

substituindo as equações (2.14) e (2.17) na eq.(2.13), é possível escrever:

$$
\mathbf{u}_{\mathrm{e}}^{\mathrm{T}} \mathbf{k}_{\mathrm{e}} \mathbf{u}_{\mathrm{e}}=\int_{\mathrm{v}_{\mathrm{e}}}\left(\mathbf{B} \mathbf{u}_{\mathrm{e}}\right)^{\mathrm{T}} \mathbf{C}_{\mathrm{e}}\left(\mathbf{B} \mathbf{u}_{\mathrm{e}}\right) \mathrm{dv} \mathrm{v}_{\mathrm{e}} \Rightarrow \mathbf{u}_{\mathrm{e}}^{\mathrm{T}} \mathbf{k}_{\mathrm{e}} \mathbf{u}_{\mathrm{e}}=\int_{\mathrm{v}_{\mathrm{e}}}\left(\mathbf{u}_{\mathrm{e}}^{\mathrm{T}} \mathbf{B}^{\mathrm{T}}\right) \mathbf{C}_{\mathrm{e}}\left(\mathbf{B} \mathbf{u}_{\mathrm{e}}\right) \mathrm{dv} v_{\mathrm{e}} \Rightarrow
$$




$$
\mathbf{u}_{\mathrm{e}}^{\mathrm{T}} \mathbf{k}_{\mathrm{e}} \mathbf{u}_{\mathrm{e}}=\mathbf{u}_{\mathrm{e}}^{\mathrm{T}}\left(\int_{\mathrm{v}_{\mathrm{e}}} \mathbf{B}^{\mathrm{T}} \mathbf{C}_{\mathrm{e}} \mathbf{B} \mathrm{dv}_{\mathrm{e}}\right) \mathbf{u}_{\mathrm{e}} \Rightarrow \mathbf{k}_{\mathrm{e}}=\int_{\mathrm{v}_{\mathrm{e}}} \mathbf{B}^{\mathrm{T}} \mathbf{C}_{\mathrm{e}} \mathbf{B} \mathrm{dv} \mathbf{v}_{\mathrm{e}}
$$

assim, a integral no volume, representada pela eq.(2.18), é a matriz de rigidez do elemento finito, que, para o caso em questão, tem espessura ( $\mathrm{t}_{\mathrm{e}}$ ) constante. Logo, a eq.(2.18) pode ser reescrita na forma da integral de área de acordo com a seguinte equação:

$$
\mathbf{k}_{\mathrm{e}}=\int_{\mathrm{A}_{\mathrm{e}}} \mathbf{B}^{\mathrm{T}} \mathbf{C}_{\mathrm{e}} \mathbf{B} \mathrm{t}_{\mathrm{e}} \mathrm{dA} \mathrm{A}_{\mathrm{e}}
$$

onde $A_{e}$ é a área do elemento.

Para expressar d $A_{e}$ em função das coordenadas locais (r, s), a eq.(2.19) deve ser alterada conforme mostra a eq.(2.20).

$$
\mathbf{k}_{\mathrm{e}}=\int_{-1}^{1} \int_{-1}^{1} \mathbf{B}^{\mathrm{T}} \mathbf{C}_{\mathrm{e}} \mathbf{B} \mathrm{t}_{\mathrm{e}}(\operatorname{det} \mathbf{J}) \mathrm{ds} \mathrm{dr}
$$

Na eq.(2.20), J é a matriz Jacobiana definida conforme a eq.(2.21).

$$
\mathbf{J}=\left[\begin{array}{ll}
\frac{\partial x}{\partial r} & \frac{\partial y}{\partial r} \\
\frac{\partial x}{\partial s} & \frac{\partial y}{\partial s}
\end{array}\right]
$$

O uso das funções de forma, expressas por meio das coordenadas locais, permite-nos trabalhar com elementos quadriláteros mais genéricos. Isso é possível devido à aplicação da matriz Jacobiana, que relaciona as coordenadas locais com as coordenadas globais. Contudo, a função resultante no interior da integral dupla, vide eq.(2.20), é de difícil integração através dos métodos analíticos. Em face deste problema, o cálculo da matriz de rigidez do elemento se dá numericamente, por meio do método da Quadratura de Gauss-Legendre (BATHE, 1996). 


\subsubsection{Determinação dos deslocamentos nodais da estrutura}

Conhecendo as matrizes de rigidez de todos os elementos da malha, faz-se necessário montar a matriz de rigidez da estrutura ou matriz de rigidez global $\left(\mathbf{k}_{\mathrm{g}}\right)$. O processo se dá por meio de uma matriz intermediária, chamada de matriz de conectividade, que relaciona os graus de liberdade locais de cada elemento aos graus de liberdade globais da estrutura. Maiores detalhes sobre esta sistemática de montagem da matriz de rigidez global podem ser encontrados em Bathe (1996).

Sendo o carregamento externo à estrutura expresso através do vetor $\mathbf{f}_{\mathrm{g}}$, que é um dado do problema, calculam-se os deslocamentos nodais $\mathbf{u}_{\mathrm{g}}$ através da eq.(2.22).

$$
\mathbf{k}_{\mathrm{g}} \mathbf{u}_{\mathrm{g}}=\mathbf{f}_{\mathrm{g}}
$$

Neste trabalho, empregou-se o método dos gradientes conjugados (BRAESS, 2001) para solucionar a eq.(2.22). 


\subsection{MÉTODO DA OTIMIZAÇÃO TOPOLÓGICA}

\subsubsection{Conceitos iniciais}

Existem três abordagens principais para os problemas de otimização estrutural, vide Figura 2.2. Na primeira, a estrutura apresenta a forma e a topologia fixas, ou seja, variam-se as suas dimensões, tais como o diâmetro da secção transversal de seus componentes, por exemplo. Este tipo de abordagem é chamado de otimização paramétrica (VANDERPLAATS, 1984).

$\mathrm{Na}$ segunda abordagem, um pouco mais genérica, a estrutura apresenta a forma variável e a topologia fixa - não ocorre a inclusão de "buracos" -, trata-se da otimização de forma (HAFTKA; GRANDHI, 1986). Neste método, as variáveis de projeto definem o contorno do domínio, cuja forma é alterada a cada iteração do processo de otimização. Isso representa um inconveniente, pois, a cada mudança de forma, é necessário refazer a malha de elementos finitos do domínio de projeto.

A otimização topológica (OT), terceira e última abordagem, representa uma bem sucedida alternativa para contornar esta questão do "remalhamento" do domínio de projeto. Assim como na otimização de forma, o domínio também é discretizado em elementos finitos, contudo, as suas dimensões são mantidas inalteradas ao longo de todo o processo de otimização. Este é um dos principais conceitos inerentes ao método, ou seja, trabalhar com um domínio fixo estendido (DFE) de projeto (BENDSØE; KIKUCHI, 1988). Na busca da solução "ótima", distribui-se o material por todo o domínio. Isso é feito de tal forma que se possa otimizar um determinado critério de custo - como o da máxima rigidez com o menor peso, por exemplo. Na otimização topológica, obtém-se os melhores resultados, pois a inclusão de "buracos" confere à estrutura um melhor desempenho se comparado às outras duas abordagens. 

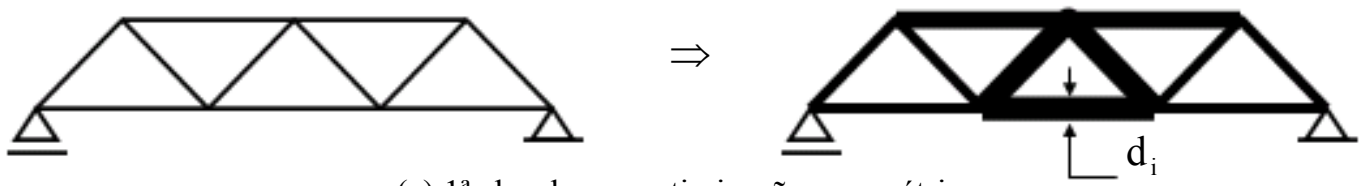

(a) $1^{\text {a }}$ abordagem: otimização paramétrica

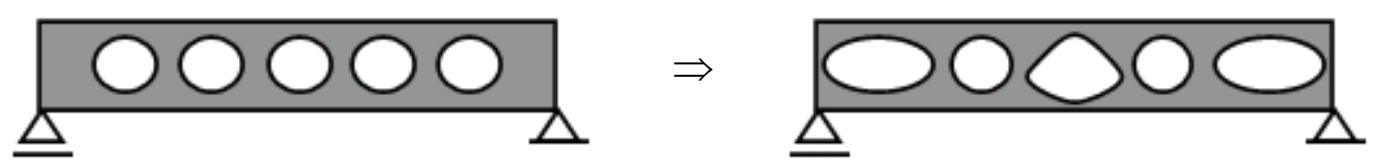

(b) $2^{\mathrm{a}}$ abordagem: otimização de forma
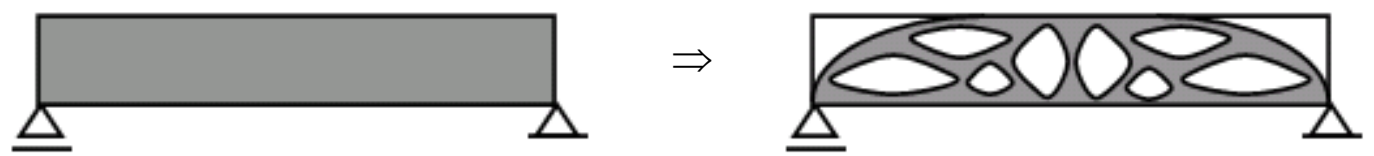

(c) $3^{\text {a }}$ abordagem: otimização topológica

Figura 2.2 - Principais abordagens empregadas na otimização estrutural.

Por conta destas vantagens, o MOT vem se estabelecendo na indústria automotiva e aeronáutica (BENDSØE; SIGMUND, 2003). Mais recentemente, tem sido muito explorado no projeto de MEMS, principalmente na síntese de mecanismos flexíveis (LARSEN; SIGMUND; BOUWSTRA, 1997). Como já mencionado, é necessário estabelecer um domínio estendido fixo para o projeto de uma estrutura que otimize um determinado critério de custo, ou desempenho. As forças e as condições de contorno associadas também devem ser definidas para completar o modelo. Através de um software de elementos finitos, o domínio fixo é discretizado e um arquivo de texto - contendo todas as informações do modelo - é gerado. Um código de otimização topológica, capaz de otimizar o critério de custo desejado, lê o arquivo de texto. Na seqüência, este código executa as iterações distribuindo a quantidade limitada de material pelo domínio discretizado. Em cada iteração, um método de otimização encontra a solução de um problema cujas variáveis determinam a existência, ou não, de material nos elementos finitos da malha. Este problema utiliza os resultados obtidos da análise de elementos finitos do domínio fixo de projeto. A convergência da solução resulta na topologia de uma estrutura com "buracos". Normalmente, a imagem desta topologia fornece um resultado primário, uma vez que o nível de refinamento da malha de elementos finitos e a 
possível presença de regiões, ou detalhes, decorrentes das instabilidades numéricas ${ }^{2}$ do processo inviabilizam a fabricação da estrutura sintetizada. O resultado precisa ser interpretado, o que pode ser feito através de técnicas de processamento de imagem, técnicas de otimização de forma (BREMICKER et al., 1991), ou mesmo desenhando uma nova estrutura com base na topologia obtida. As modificações decorrentes da interpretação alteram o desempenho da estrutura, logo, faz-se necessário analisar, pelo método dos elementos finitos, o resultado interpretado. Todo procedimento descrito acima está esquematizado na Figura 2.3.

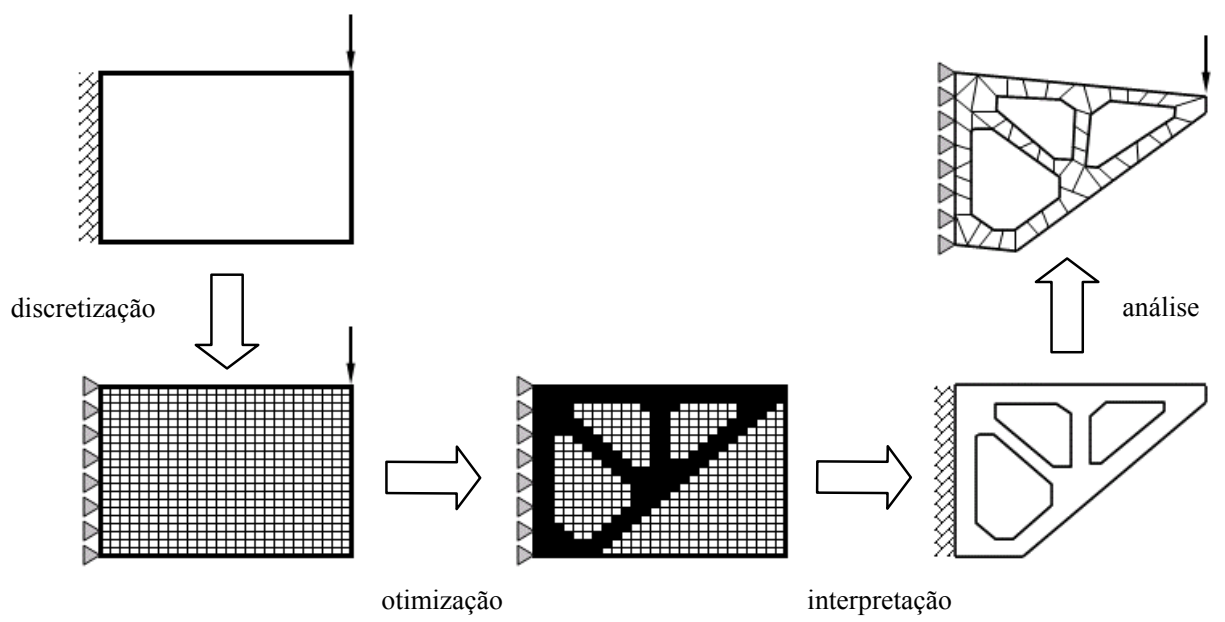

Figura 2.3 - Etapas relacionadas ao método da otimização topológica.

\subsubsection{Histórico}

Nos trabalhos iniciais relacionados à otimização estrutural (MICHELL, 1904), têm-se estruturas formadas por infinitas barras rígidas separadas por um espaço infinitesimal, conforme se vê na Figura 2.4, na qual o resultado corresponde a uma estrutura otimizada onde as barras acompanham as linhas das isotensões principais definidas segundo a teoria clássica da elasticidade.

\footnotetext{
${ }^{2}$ As instabilidades numéricas mais comuns associadas aos problemas de OT são a dependência de malha e a instabilidade de tabuleiro. No caso particular do projeto de mecanismos flexíveis há também o problema das "dobradiças". Todas estas instabilidades serão discutidas ao longo do texto.
} 


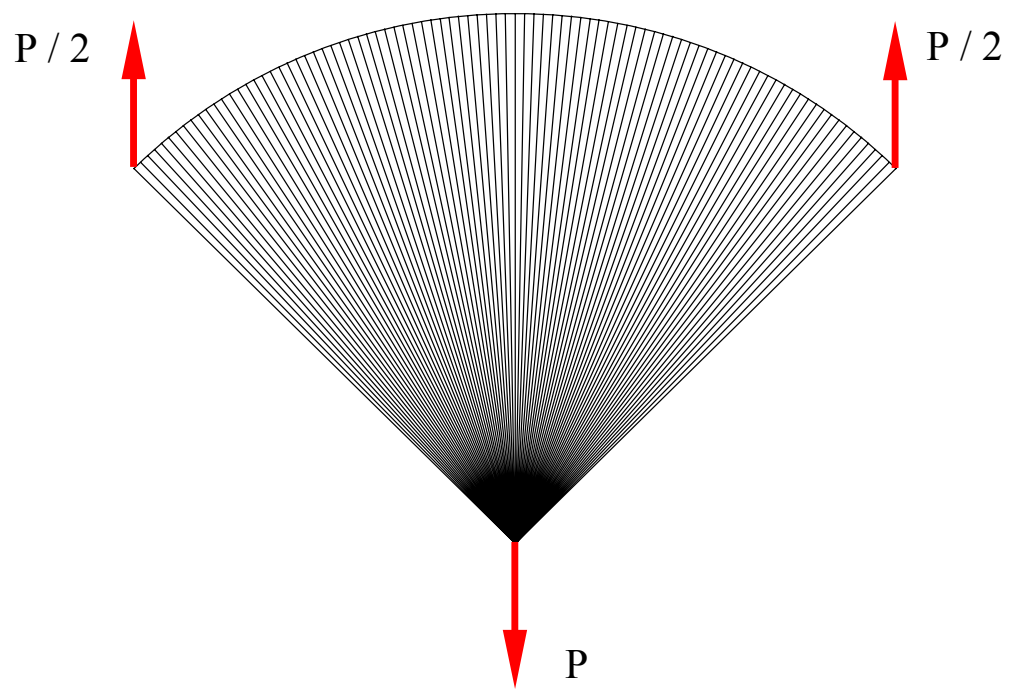

Figura 2.4 - Representação de uma estrutura resultante dos trabalhos de Michell.

Os trabalhos de Michell (1904), baseados em resultados como este, motivaram os estudos relacionados com a otimização de estruturas a partir de um domínio discretizado em um número finito de barras. Trabalhos nesse sentido podem ser vistos em (ROZVANY; ZHOU, 1991; ROZVANY; BENDSØE; KIRSCH, 1995).

Com o método da homogeneização proposto por Bendsøe e Kikuchi (1988), a otimização topológica, a partir de meios contínuos, ganhou força em relação à otimização de forma. A idéia tem uma forte correlação com os estudos de Cheng e Olhoff (1981), que investigaram a natureza do problema correspondente à maximização da rigidez de placas delgadas considerando a espessura como variável de projeto. Nestes estudos, constatou-se que o problema apresentava múltiplos "ótimos" locais, não havendo um domínio matemático fechado capaz de oferecer uma solução. Como no caso das placas, na otimização topológica, este comportamento também se manifesta através da dependência que a solução apresenta em relação ao nível de discretização imposto ao problema contínuo (dependência de malha). Bendsøe e Kikuchi (1988) consideraram a microestrutura de material para resolver o problema da otimização topológica aplicada à maximização da rigidez de estruturas com o volume restringido. Este recurso da microestrutura já havia sido empregado por Cheng (1981) na solução do problema das placas. A idéia consiste em variar os parâmetros dimensionais de uma célula unitária que representa a referida microestrutura. Deste modo, é possível fechar o domínio matemático do problema e garantir a existência de uma solução. Este conceito é a base do MOT efetuado pelo método da homogeneização, cujos detalhes serão expostos na seção 2.3.4. 
Conforme será discutido na seção 2.3.3, através da utilização do método da restrição de perímetro estudado por Ambrosio e Buttazzo (1993) e, posteriormente, por Haber; Jog e Bendsøe (1996), o problema de OT aplicado à maximização da rigidez estrutural também é solucionável. Contudo, devido ao domínio de solução mais restrito, os resultados encontrados são "sub-ótimos" se comparados àqueles obtidos pelo método da homogeneização. Ainda que isso não seja um inconveniente em relação aos problemas de Engenharia, a aplicação da restrição de perímetro tornou-se menos usual, pois apresenta maiores dificuldades à implementação numérica.

A partir do trabalho de Bendsøe e Kikushi (1988), vários outros se sucederam. Thomsen (1992) propôs uma extensão do método da homogeneização estudando a otimização de estruturas compostas por mais de um material. Ma; Kikuchi e Cheng (1995) usaram a otimização topológica para maximizar a freqüência de ressonância de estruturas obtidas a partir de meios contínuos. O problema da instabilidade estrutural devido à flambagem também foi solucionado através do MOT (NEVES; RODRIGUES; GUEDES, 1995). Fukushima; Suzuki e Kikuchi (1991) fizeram a aplicação do MOT para reforçar chapas usadas na armação de carrocerias de automóveis. As aplicações da OT se espandem a outras áreas, além daquelas relacionadas às estruturas mecânicas clássicas empregadas na Engenharia, assim, o MOT se mostra como uma ferramenta muito adequada ao projeto de dispositivos tais como os mecanismos flexíveis (ANANTHASURESH; KOTA, 1995; SIGMUND, 1997; NISHIWAKI et al., 2001), os quais têm grande utilização nos MEMS. Da lista de aplicações do MOT, pode-se ainda citar: o projeto de transdutores piezelétricos (SILVA; FONSECA; KIKUCHI, 1997, 1998), a maximização da condutividade térmica na transferência de calor (PARK, 1995), o projeto de micro-mecanismos flexíveis com atuação térmica ou elétrica (JONSMANN; SIGMUND; BOUWSTRA, 1999; SIGMUND, 2001b; SIGMUND, 2001c), o projeto de atuadores flextensionais piezelétricos (SILVA et al., 1999; SILVA; NISHIWAKI; KIKUCHI, 2000; CANFIELD; FRECKER, 2000), além de outras várias possibilidades para o uso do método. Uma revisão sobre as aplicações do MOT pode ser vista em Bendsøe e Sigmund (2003). 


\subsubsection{Dependência de malha}

O objetivo da otimização topológica consiste em determinar os "vazios" e a conectividade da estrutura constituída de um material isotrópico (material base). Assim, a sua topologia pode ser escrita segundo a função discreta representada pela eq.(2.23).

$$
\rho(\mathbf{x})=\left\{\begin{array}{c}
1 \text { se } \mathbf{x} \in \Omega^{\mathrm{mat}} \\
0 \text { se } \mathbf{x} \in \Omega \backslash \Omega^{\mathrm{mat}}
\end{array}\right.
$$

Na eq.(2.23), $\rho$ é a densidade ${ }^{3}$ do ponto $\mathbf{x}$ pertencente ao domínio fixo $\Omega$, cujo subdomínio $\Omega^{\text {mat }}$ é ocupado pelo material base. A Figura 2.5 ilustra uma possível representação gráfica para a eq.(2.23).

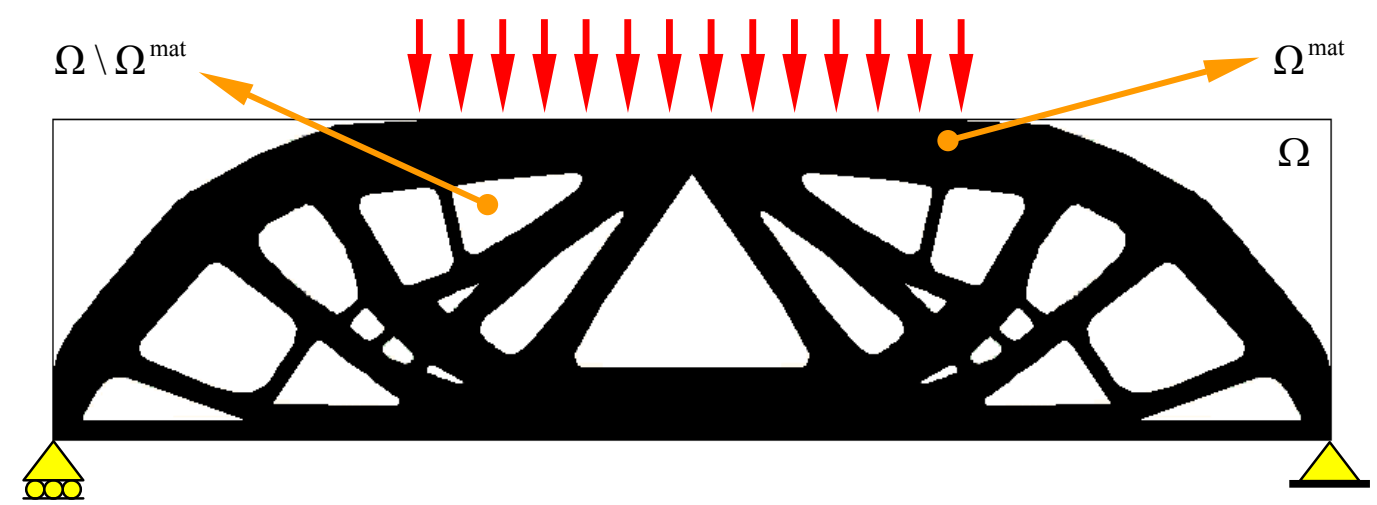

Figura 2.5 - Exemplo de distribuição de material segundo a parametrização discreta.

Sendo $\mathbf{E}^{0}$ o tensor constitutivo do material base, $\mathbf{E}$ representa as propriedades elásticas de um ponto $\mathbf{x}$ qualquer do domínio fixo $\Omega$, vide eq.(2.24).

$$
\mathbf{E}(\mathbf{x})=\rho(\mathbf{x}) \mathbf{E}^{0}
$$

O problema da maximização da rigidez de estruturas com o volume restringido, colocado conforme as equações (2.23) e (2.24), é mal-posto, ou seja, não apresenta solução para a parametrização discreta $(0-1)$ das variáveis de projeto (ALLAIRE, 2002;

\footnotetext{
${ }^{3}$ A palavra "densidade" é considerada, neste texto, como sendo a densidade relativa do material parametrizado em relação ao material base. Pode-se dizer que se trata de uma "pseudo-densidade".
} 
PETERSSON; SIGMUND, 1998). Nestas condições, o domínio matemático do problema não é fechado, assim, a despeito dos múltiplos "ótimos" locais existentes, não há um "ótimo" global. Intuitivamente, pode-se compreender tal comportamento matemático através da observação de uma estrutura submetida a um carregamento externo. A medida em que se acrescentam mais furos pela estrutura, desde que o volume seja mantido constante, a sua rigidez é incrementada. Matematicamente, é possível aumentar indefinidamente a quantidade de furos, variando-se, inclusive, os seus formatos. Pode-se assim, obter valores cada vez maiores com respeito à rigidez da estrutura. Do ponto de vista da solução numérica do problema, a medida em que se refina a malha de elementos finitos permite-se que a topologia apresente mais e menores furos, daí a dependência da solução com relação ao nível de discretização do domínio fixo de projeto. Este comportamento pode ser observado no exemplo demonstrado pela Figura 2.6.

(a)

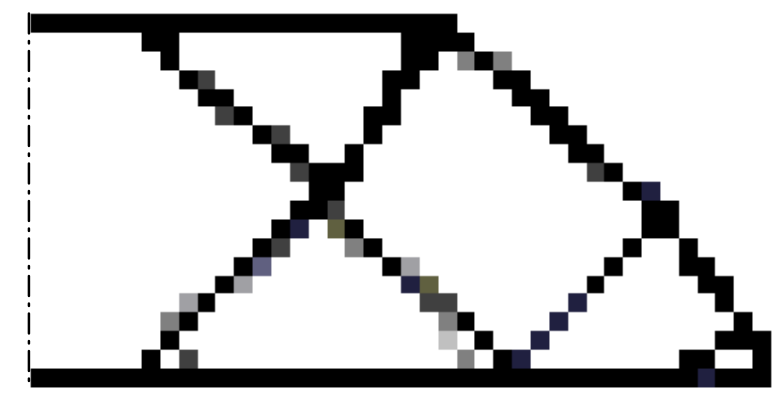

(b)

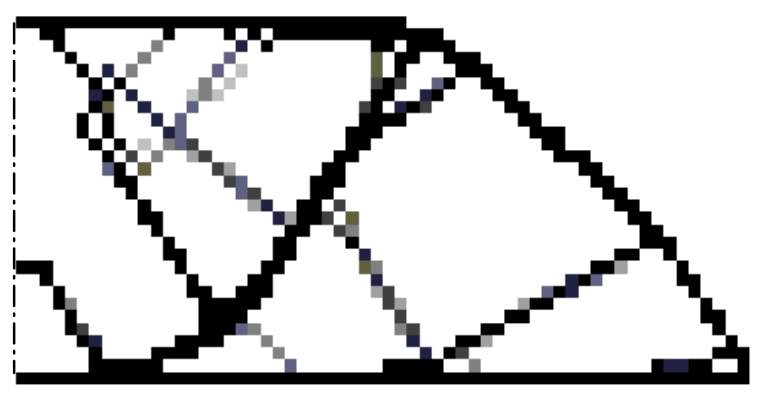

Figura 2.6 - (a) representação da metade de uma viga simétrica obtida a partir de uma discretização de 800 elementos finitos; (b) novo resultado obtido a partir de uma discretização de 1800 elementos finitos.

Ambrosio e Buttazzo (1993) propuseram o conceito da restrição de perímetro da estrutura para restringir o espaço de solução do problema discreto (0-1) e, desta forma, alcançar a solução do problema de OT. Respaldados por essa idéia, Haber; Jog e Bendsøe (1996) aplicaram a restrição de perímetro em seus trabalhos, considerando severas 
penalizações no uso do método $\mathrm{SIMP}^{4}$. Com isso, os resultados numéricos indicaram que o problema de fato se tornou bem-posto. Uma outra possibilidade para restringir o espaço de solução se dá através do controle dos gradientes das variáveis de projeto. Niordson (1983) demonstrou que a restrição na variação da espessura de chapas delgadas era capaz de resolver o problema da não existência de solução para a otimização da rigidez. Analogamente, problemas de OT baseados no método SIMP foram solucionados através do controle da variação das densidades (PETERSSON; SIGMUND, 1998). Apesar de se mostrarem eficientes na obtenção da solução, a restrição de perímetro e o controle dos gradientes são difíceis de serem tratados numericamente, assim, poucos trabalhos seguiram essas abordagens.

\subsubsection{Modelos de materiais}

Além dos métodos restritivos descritos na seção anterior, uma outra forma de garantir a existência da solução para os problemas de OT consiste em permitir que as densidades possam assumir valores compreendidos entre 0 (zero) e 1 (um). Com isso, o problema é relaxado, podendo haver a presença de materiais intermediários distribuídos pelo domínio fixo (BENDSØE; SIGMUND, 1999). A parametrização contínua do tensor constitutivo de material garante a existência da solução para o problema (BENDSØE, 1995), contudo, a maneira pela qual a variação ocorre é definida pelo modelo de material adotado. Os dois principais modelos de materiais que definem a mistura em micro-escala entre dois ou mais materiais são o método da homogeneização (BENDSØE; KIKUCHI, 1988; GUEDES; KIKUCHI, 1990; SUZUKI; KIKUCHI, 1991) e o método das densidades (BENDSØE; SIGMUND, 1999).

\section{Método da homogeneização}

No método da homogeneização são definidas as propriedades elásticas dos pontos do domínio fixo como se cada um deles fosse constituído de um material resultante da composição de dois materiais isotrópicos, sendo usualmente considerados o ar ("vazio") e o

\footnotetext{
${ }^{4}$ Assim como o método da homogeneização, o método SIMP (Simple Isotropic Material with Penalization) também parametriza as variáveis de projeto de forma continua. Seus detalhes serão expostos na seção 2.3.4.
} 
material base (sólido). Com isso, as propriedades elásticas efetivas (ou homogeneizadas) de um dado ponto equivalem àquelas observadas num material formado pela repetição periódica de uma célula unitária, vide Figura 2.7. Suzuki e Kikuchi (1991), além de Bendsøe e Kikuchi (1988), utilizaram um padrão de célula unitária que pode ser representada por um quadrado de lado unitário dotado de um furo retangular de lados $a$ e $b$. O furo pode ainda apresentar uma rotação $\theta$, conforme mostra a Figura 2.7. Neste caso, as variáveis de projeto do problema de OT são os parâmetros $a, b$ e $\theta$, logo, durante o processo de otimização, faz-se necessário calcular os gradientes das propriedades elásticas em relação a estas variáveis, o que pode ser feito por meio de uma tabela, previamente determinada, que relaciona tais propriedades a uma gama de combinações considerando um grande número de valores discretos para $a$ e $b$. Estas propriedades elásticas são expressas por meio do tensor constitutivo de material que é rotacionado em função de $\theta$.

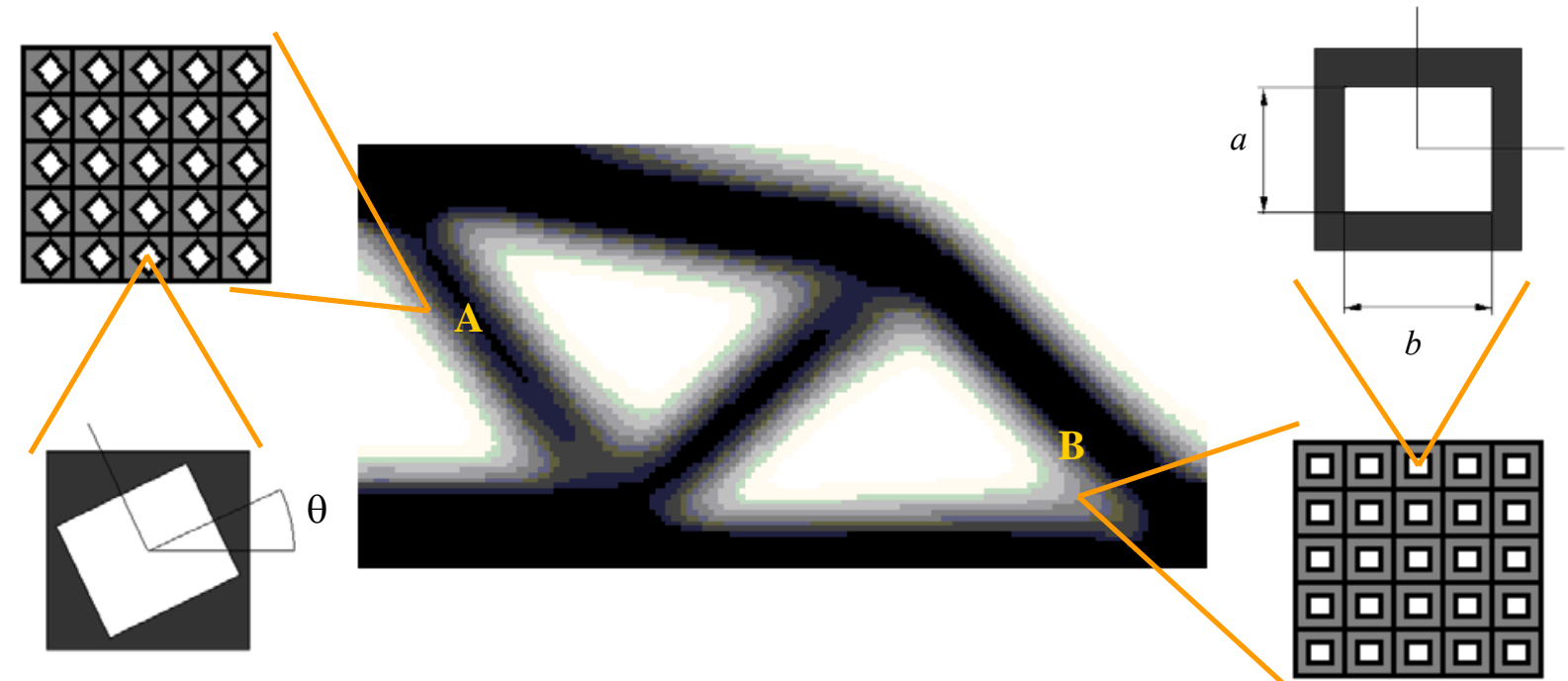

Célula unitária $\operatorname{com} \theta>0^{\circ}$

Material que representa as propriedades elásticas do ponto B

Figura 2.7 - Representação esquemática de uma distribuição de material através da homogeneização.

Com a parametrização dimensional da célula unitária, têm-se a definição da sua densidade volumétrica $(\rho)$, cujo valor está compreendido entre 0 (zero) e 1 (um). Contudo, de acordo com o que foi discutido anteriormente, o tensor constitutivo de material não é função direta da densidade da célula, mas sim, de seus parâmetros dimensionais. Ainda com respeito às microestruturas, elas podem ser classificadas de acordo com a capacidade de relaxar o 
problema discreto. As microestruturas ditas "sub-ótimas" relaxam o problema parcialmente, já as microestruturas “ótimas” garantem a relaxação completa (ALLAIRE; AUBRY, 1999). No caso da maximização da rigidez (minimização da flexibilidade média), a relaxação completa é possível através das microestruturas laminadas, conhecidas na literatura como rank-n (ALLAIRE; KOHN, 1993; ALLAIRE; AUBRY, 1999). São microestruturas que possuem $n$ camadas de laminação, cada uma das quais constituída por uma sub-camada igualmente laminada. Maiores detalhes sobre a configuração das microestruturas rank- $n$ e o cálculo das propriedades efetivas dos materias relacionados podem ser encontrados em (BENDSØE; SIGMUND, 2003). Uma revisão sobre o método da homogeneização é apresentada em Hassani e Hinton (1998).

Método das densidades

Como alternativa à homogeneização, Bendsøe (1989) propôs o método das densidades, no qual uma única variável de projeto $(\rho)$ é utilizada para parametrizar o tensor constitutivo através da eq.(2.24). Para tanto, considera-se o intervalo $0 \leq \rho \leq 1$ ao invés da eq.(2.23). Este modelo de material não leva em conta os detalhes da microestrutura, contudo, para o caso da maximização da rigidez, determina topologias similares àquelas obtidas por meio do método da homogeneização quando microestruturas "ótimas" são escolhidas (rankn). Nos dois métodos, os resultados apresentam uma extensa área com densidades intermediárias (escalas de cinza), o que dificulta a interpretação e fabricação da estrutura obtida. Como forma de reduzir a presença destas escalas de cinza, a densidade é penalizada. Assim, a parametrização do tensor constitutivo passa a ser:

$$
\mathbf{E}(\mathbf{x})=[\rho(\mathbf{x})]^{\mathrm{n}} \mathbf{E}^{0}, \quad \rho \in[0,1] \text { e } \mathbf{x} \in \Omega
$$

onde n é um número denominado coeficiente de penalização, cujo valor deve ser maior do que 1 (um). O modelo de material segundo a eq.(2.25) é conhecido na literatura por Simple Isotropic Material with Penalization (SIMP) (BENDSØE, 1989; ROZVANY; ZHOU; BIRKER, 1992) e tem larga utilização nos trabalhos sobre OT. Porém, deve-se considerar que, na medida em que $\mathrm{n}$ assume valores maiores, o problema recupera o seu caráter discreto (RIETZ, 2001), retornando ao espaço de solução aberto. Assim, como forma de tornar o problema bem-posto, o método SIMP deve ser utilizado em conjunto com outros esquemas 
numéricos, tais como as restrições de perímetro e as restrições dos gradientes das variáveis de projeto. Para determinar os valores do coeficiente de penalização (n), há uma discussão interessante e que está associada à existência dos materiais cujas propriedades elásticas estão definidas segundo a parametrização feita pelo método SIMP. Bendsøe e Sigmund (1999) demonstraram que tais materiais, determinados a partir da mistura entre o ar ("vazio") e um material base isotrópico, são fisicamente factíveis, se as inequações (2.26) e (2.27), válidas para o estado plano de tensões, forem satisfeitas.

$$
\begin{aligned}
& \rho^{\mathrm{n}} \mathrm{E}^{0} \leq \frac{\rho \mathrm{E}^{0}}{3-2 \rho}, \text { sendo } \rho \in[0,1] \\
& \mathrm{n} \geq \max \left\{\frac{2}{1-v}, \frac{4}{1+v}\right\}
\end{aligned}
$$

As inequações (2.26) e (2.27), para as quais $v$ é o coeficiente de Poisson do material composto, são baseadas nos limites de Hashin e Shtrikman (1963), que definiram as condições para determinar se as propriedades obtidas a partir das microestruturas formadas por dois materiais isotrópicos são fisicamente possíveis. No caso do aço $(v=1 / 3)$, deve-se ter $n \geq 3$ para que as inequações acima sejam satisfeitas. Assim, se por um lado, valores de $n$ superiores a 3 (três) garantem a existência do material composto, por outro, comprometem o problema de OT, tornando-o mal-posto. Isso sugere, uma vez mais, que a aplicação do método SIMP seja feita conjuntamente com alguma forma de controle da complexidade da topologia.

\subsubsection{Filtros}

Os métodos de filtragem apresentam a vantagem de serem mais fáceis quanto à implementação e mais rápidos - em termos computacionais - do que as restrições de perímetro e as restrições dos gradientes das variáveis de projeto e, assim como estes métodos, também são capazes de restringir o espaço de solução dos problemas onde se utiliza o método SIMP com maiores valores para o coeficiente de penalização. Filtrar consiste em aplicar um 
operador matemático sobre uma função não regular, com isso, obtém-se uma nova função mais suave (CARDOSO, 2000). Experimentalmente, constata-se que um esquema de filtragem aplicado sobre os gradientes da função objetivo se apresenta como um meio de alcançar a independência de malha (SIGMUND, 1994; SIGMUND, 1997). Na OT, os filtros de densidade também são capazes de proporcionar o controle dos gradientes das variáveis de projeto (BOURDIN, 2001), não de uma maneira tão efetiva como na utilização de restrições diretas, mas, no caso dos filtros espaciais de densidade (CARDOSO; FONSECA, 1999; CARDOSO; FONSECA, 2003), consegue-se bons resultados quanto à solução do problema da dependência de malha. A idéia básica consiste no fato de que as densidades dos elementos finitos da malha, cujos centróides estejam na região interna de uma circunferência de centro coincidente ao centróide do elemento central, influenciam na densidade deste último elemento. Um conceito diferente fundamenta os filtros de vizinhança fixa (SWAN; KOSAKA, 1997), onde a influência sobre um elemento se dá apenas pelos seus vizinhos adjacentes. Assim, diferentemente dos filtros espaciais, os filtros de vizinhança não resolvem o problema da dependência de malha, sendo úteis apenas para solucionar o problema da instabilidade de tabuleiro, que será o assunto da seção 2.3.6. Maiores detalhes sobre os operadores matemáticos e os procedimentos computacionais correspondentes a estes dois tipos de filtragem para as densidades serão apresentados no capítulo 3. É importante lembrar que estes esquemas numéricos, possíveis de serem utilizados conjuntamente com a parametrização baseada no método SIMP, determinam soluções "sub-ótimas" em relação ao problema completamente relaxado. Contudo, apesar de serem "sub-ótimos", estes resultados atendem às necessidades exigidas pelos problemas de Engenharia, sendo, portanto, aceitos para este fim.

\subsubsection{Instabilidade de tabuleiro}

Para um grande número de trabalhos em que a OT se aplica, pode-se observar regiões onde elementos com e sem material (pretos e brancos) se alternam num padrão semelhante a um tabuleiro de xadrez. Na literatura, esta instabilidade numérica é conhecida como checkerboard, neste texto, é denominada instabilidade de tabuleiro, vide Figuras 2.8 e 2.9a. 


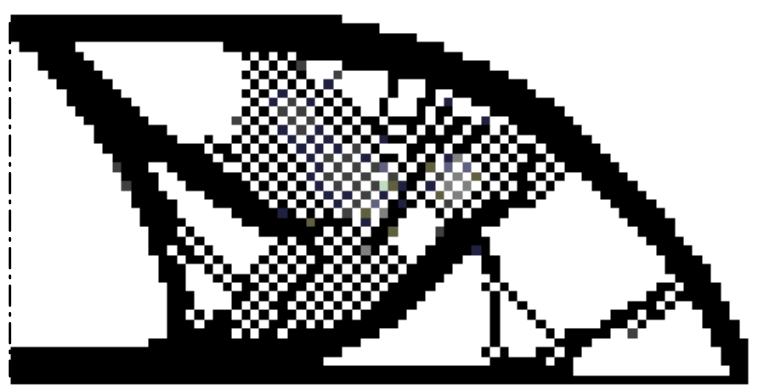

Figura 2.8 - Ocorrência da instabilidade de tabuleiro.

A presença da instabilidade de tabuleiro é indesejável, pois não se trata de um aspecto que confira à solução uma forma natural de melhorar o desempenho do resultado, mas sim, de um problema associado a implementação numérica. Apesar de existirem muitos trabalhos que procuram estudar a formação da instabilidade de tabuleiro, não há um consenso quanto à melhor forma de explicar as causas do problema, muito embora, duas abordagens tenham mais força e aceitação. Em uma primeira explicação, Diaz e Sigmund (1995) propõem que o problema seria causado pelas aproximações numéricas introduzidas pelo método dos elementos finitos. Segundo estes autores, a utilização de elementos isoparamétricos de quatro nós, com funções de interpolação bi-lineares, confere à distribuição mostrada na Figura 2.9a uma rigidez artificialmente maior, em termos de deformação ao cisalhamento, quando comparada à rigidez obtida com a distribuição homogênea representada pela Figura 2.9b, levando-se em conta que, em ambos os casos, o volume de material é o mesmo.

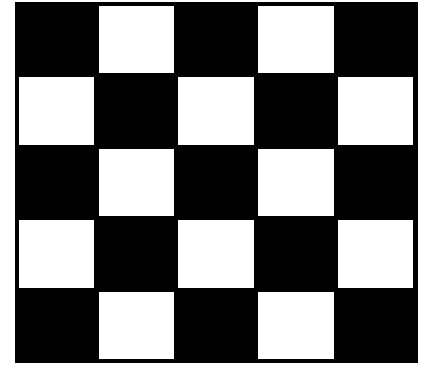

(a) Instabilidade de tabuleiro.

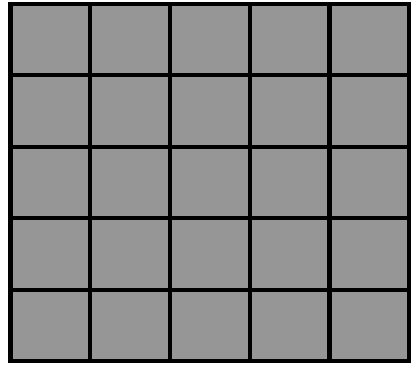

(b) Distribuição homogênea.

Figura 2.9 - Distribuição de material.

Assim, no problema da máxima rigidez com restrição de volume, bem como, nos problemas em que determinadas regiões precisam apresentar rigidez mais elevada como forma de atender à formulação, a instabilidade de tabuleiro é a melhor "opção" do otimizador. 
Elementos finitos de ordem superior seriam capazes de proporcionar uma distribuição mais real do campo dos deslocamentos, e portanto, poderiam reduzir a ocorrência do problema.

A segunda explicação, por sua vez, está relacionada ao fato de que, no MOT, resolvese um problema de variacional misto, no qual se distribui dois campos físicos, o campo dos deslocamentos e o campo das densidades. Um comportamento de instabilidade similar pode ser observado na distribuição das pressões para o problema de escoamento de fluídos de Stokes (ODEN; KIKUCHI; SONG, 1982), tal distribuição é obtida através da análise por elementos finitos e também envolve dois campos físicos, neste caso, tem-se os campos das velocidades e das pressões. Baseados nesta experiência, Jog e Haber (1996) utilizaram a teoria do método dos elementos finitos mistos para expressar o problema da OT de forma similar ao problema de Stokes. Assim, definiram um sistema não-linear envolvendo os campos dos deslocamentos e das densidades. Como resultado deste trabalho, os autores propõem que, para uma gama de combinações entre tipos de elementos, com os quais se pode interpolar os dois campos físicos, a instabilidade de tabuleiro deixa de estar presente na solução do problema. Contudo, todas as combinações demandam elementos de alta ordem, ao menos para interpolar os deslocamentos (no mínimo 8 nós).

Pela perspectiva destas duas correntes (DIAZ; SIGMUND, 1995; JOG; HABER, 1996), a adoção de elementos finitos de ordem superior seria necessária, sendo que para Jog e Haber (1996), isto não seria suficiente caso fosse feito apenas para o campo dos deslocamentos, já que seria necessário também interpolar o campo das densidades de forma adequada. De qualquer modo, a utilização de elementos finitos com mais nós implicaria no aumento do custo computacional. Assim, os filtros espaciais de densidades (CARDOSO; FONSECA, 1999; CARDOSO; FONSECA, 2003), além de minimizarem o problema da dependência de malha, apresentam-se como uma solução economicamente mais viável para a redução da instabilidade de tabuleiro. Esta dupla utilidade se dá, pois, na solução do problema discreto, assim como na instabilidade de tabuleiro, ocorrem as mesmas variações abruptas do campo das densidades. Os filtros de vizinhança fixa (SWAN; KOSAKA, 1997), a despeito de serem dependentes do nível de discretização da malha, também são capazes de reduzir o problema, já que têm por objetivo suavizar a distribuição das densidades.

Os métodos de restrição de perímetro (HABER; JOG; BENDSØE, 1996) e de controle dos gradientes (PETERSSON; SIGMUND, 1998), da mesma forma que os filtros de densidade, também podem eliminar a presença da instabilidade de tabuleiro através do controle que impõe à topologia, contudo, como dito anteriormente, o tratamento numérico nestes casos é bem mais complicado do que o exigido pelos filtros de densidade. 


\subsection{OTIMIZAÇÃO TOPOLÓGICA NO PROJETO DE MECANISMOS FLEXÍVEIS}

Conforme dito anteriormente, existem duas abordagens para o projeto de mecanismos flexíveis: a síntese cinemática (HER; MIDHA, 1987; MIDHA; NORTON; HOWELL, 1992) e a síntese a partir de um meio contínuo. É na última abordagem que a OT se apresenta como uma alternativa viável. Ananthasuresh; Kota e Gianchandani (1994) foram os primeiros a estender as técnicas da otimização topológica ao projeto de mecanismos flexíveis. Naquele trabalho, considerando a maximização da rigidez, o dispositivo era projetado para atender a certos deslocamentos prescritos em determinados pontos do domínio. Dada a similaridade com o problema da otimização da rigidez estrutural, os mecanismos obtidos se mostravam muito rígidos para as aplicações de flexibilidade. Sigmund (1997) propôs uma forma de obter mecanismos flexíveis baseados na rigidez de uma peça de trabalho, que, conforme mostra a Figura 2.10, é representada por uma mola.

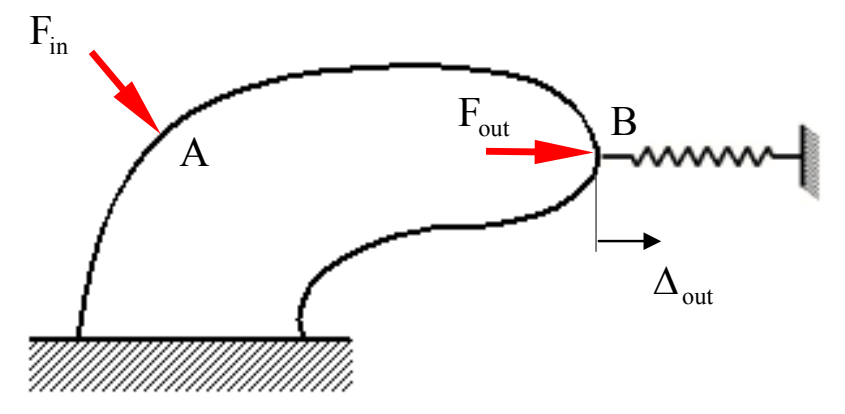

Figura 2.10 - Mola substituindo a peça de trabalho no ponto B.

Considerando pequenos deslocamentos para um sistema linear, o objetivo do problema de otimização consistia em maximizar a vantagem mecânica (VM) - mechanical advantage (MA) - que é definida pela razão entre a força aplicada sobre a mola (força observada na saída do mecanismo) e a força aplicada no ponto de entrada do mecanismo flexível. Para solucionar o problema de otimização topológica formulado, Sigmund adotou o método SIMP com um fator de penalização $n=3$, sugerindo ainda, que o controle das tensões internas do mecanismo fosse feito de forma indireta por meio da restrição do deslocamento de entrada.

Larsen; Sigmund e Bouwstra (1997) consideraram, além da vantagem mecânica, a vantagem geométrica (VG) - geometrical advantage (GA) - na função objetivo utilizada para o projeto de mecanismos flexíveis. Define-se a vantagem geométrica pela razão entre os valores absolutos dos deslocamentos de saída e entrada. A partir daí, fez-se uma analogia 
mais direta com os mecanismos de corpos rígidos, para os quais o produto da vantagem mecânica pela vantagem geométrica corresponde a 1 (um). Para o caso dos mecanismos flexíveis, este produto, chamado de eficiência mecânica (EM) - mechanical efficiency (ME) -, é necessáriamente inferior a 1 (um). Isso ocorre devido à armazenagem de parte da energia fornecida ao mecanismo no intuíto de deformá-lo. Para utilizar a formulação proposta por Larsen; Sigmund e Bouwstra (1997) é necessário especificar os valores desejados para a vantagem mecânica e para a vantagem geométrica. A minimização de uma dada função objetivo faz com que VM e VG se aproximem dos valores especificados. Essencialmente, a formulação possibilita um bom controle das relações de força e deslocamento entre a saída e a entrada do mecanismo flexível. Através da formulação proposta por Larsen; Sigmund e Bouwstra (1997), Jonsmann; Sigmund e Bouwstra (1999) projetaram um mecanismo flexível capaz de multiplicar os deslocamentos produzidos por um microatuador térmico. Desta forma, privilegiou-se a vantagem geométrica em detrimento à vantagem mecânica, ou seja, $\mathrm{VM}<1<\mathrm{VG}$.

Da mesma forma que Silva; Nishiwaki e Kikuchi (2000), Canfield e Frecker (2000) desenvolveram um meio para projetar mecanismos flexíveis capazes de transmitir os deslocamentos gerados por um atuador piezoelétrico. No entanto, estes últimos autores empregaram duas formulações distintas, comparando os resultados obtidos a partir de cada uma delas. Na primeira formulação, a função objetivo maximizava a vantagem geométrica. $\mathrm{Na}$ segunda, maximizava-se a eficiência mecânica, aproximando-a do valor unitário. Diferentemente do trabalho de Silva; Nishiwaki e Kikuchi (2000), onde as variáveis de projeto estavam relacionadas às microestruturas de material, Canfield e Frecker (2000) estabeleceram um domínio fixo de projeto discretizado em elementos de barras. Desta forma, as variáveis de projeto eram as espessuras de suas seções transversais. As soluções obtidas apresentavam diferenças sensíveis, indicando que cada formulação tinha uma aplicação específica.

Pedersen; Buhl e Sigmund (2001) propuseram uma formulação fortemente inspirada naquela definida por Sigmund (1997), contudo, ao invés de maximizar a vantagem mecânica, maximizava-se o deslocamento de saída. Para trabalhar com grandes deslocamentos, os autores implementaram uma rotina de elementos finitos capaz de lidar com a não-linearidade geométrica, assim, as equações de equilíbrio de cada um dos casos de carregamento eram solucionadas por meio de um método iterativo que levava em conta a dependência da matriz de rigidez em relação às deformações. Da mesma forma que no trabalho de Sigmund (1997), uma restrição imposta ao deslocamento do ponto de entrada de força também foi colocada na 
formulação do problema. Além de Pedersen; Buhl e Sigmund (2001), outros autores também abordaram a não-linearidade geométrica decorrente de problemas formulados para projetar mecanismos flexíveis envolvendo grandes deslocamentos (BRUNS; TORTORELLI, 2001; SIGMUND, 2001b).

O conceito de utilizar funções objetivo adequadas às aplicações dos mecanismos flexíveis foi reforçado no trabalho de Lau; Du e Lim (2001), que chamaram à atenção para as similaridades geométricas entre um mecanismo flexível e um mecanismo de corpo rígido, quando ambos tinham que desempenhar uma mesma função. Considerando uma formulação que maximizava a vantagem mecânica, foi obtido um mecanismo similar a um "balancim" amplificador de força, em contra partida, quando se utilizou uma formulação para maximizar a vantagem geométrica, a topologia tinha a aparência de um "balancim" amplificador de deslocamentos.

Uma questão importante a respeito de todas a formulações discutidas até aqui é a consideração de uma mola como forma de representar a peça para a qual o mecanismo flexível disponibilizará o trabalho mecânico. Várias outras publicações fazem esta consideração (HETRICK; KIKUCHI; KOTA, 1999; TAI; CHEE, 2000; SIGMUND, 2001a), no entanto, existem outras que descartam a mola (NISHIWAKI et al., 1998; CHEN; SILVA; KIKUCHI, 2001; NISHIWAKI et al. 2001; LIMA, 2002). Basicamente, os problemas de otimização que suprimem a mola apresentam uma função multi-objetivo que compatibiliza a flexibilidade e a rigidez do mecanismo flexível. A idéia parte do princípio de que o mecanismo deve apresentar flexibilidade suficiente para se deformar e alcançar a peça de trabalho, porém, deve também ser rígido a ponto de resistir aos trabalhos externos. Tem-se, portanto, uma solução de compromisso entre estas duas grandezas. A maximização da flexibilidade é obtida pela maximização da energia mútua (SHIELD; PRAGER, 1970; HUANG, 1971). Por outro lado, a maximização da rigidez se dá através da minimização da flexibilidade média.

Para determinar a energia mútua de um corpo constituído de material elástico submetido a dois carregamentos distintos $\mathbf{t}^{1}$ e $\mathbf{t}^{2}$, vide Figura 2.11, é necessário definir as energias mútuas $\mathrm{L}^{1}\left(\mathrm{u}^{2}\right)$ e $\mathrm{L}^{2}\left(\mathrm{u}^{1}\right)$, conforme as equações (2.28) e (2.29), respectivamente. 

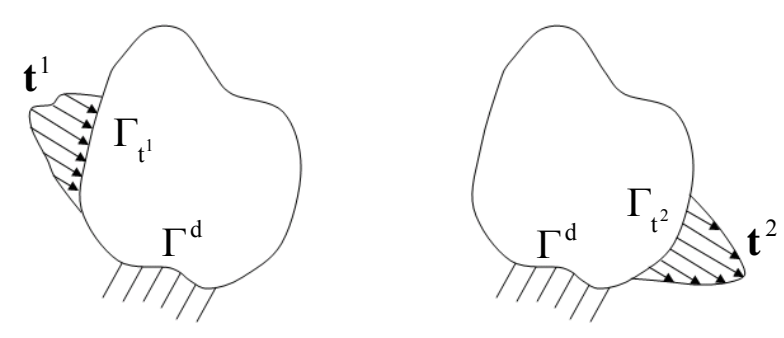

Figura 2.11 - Carregamentos distintos em um mesmo corpo.

$$
\begin{aligned}
& \mathrm{L}^{1}\left(\mathrm{u}^{2}\right)=\int_{\Gamma} \mathbf{t}^{1} \cdot \mathbf{u}^{2} \mathrm{~d} \Gamma \\
& \mathrm{L}^{2}\left(\mathrm{u}^{1}\right)=\int_{\Gamma} \mathbf{t}^{2} \cdot \mathbf{u}^{1} \mathrm{~d} \Gamma
\end{aligned}
$$

O tensor $\mathbf{u}^{1}$, obtido no estado de equilíbrio, corresponde ao campo dos deslocamentos decorrentes do carregamento $\mathbf{t}^{1}$. O tensor $\mathbf{u}^{2}$, também obtido no estado de equilíbrio, representa o campo dos deslocamentos devido ao carregamento $\mathbf{t}^{2}$. Nishiwaki et al. (1998) mostram a validade da eq.(2.30).

$$
\mathrm{E}_{\text {mútua }}=\mathrm{L}^{1}\left(\mathrm{u}^{2}\right)=\mathrm{L}^{2}\left(\mathrm{u}^{1}\right)
$$

Para projetar um mecanismo flexível através de uma função multi-objetivo, via de regra, admite-se uma força concentrada no seu ponto de entrada, o que caracterizaria o primeiro caso de carregamento. Uma força fíctícia concentrada no seu ponto de saída caracterizaria o segundo caso de carregamento. Considerando que esta força fíctícia tem o módulo unitário, a mesma direção e o mesmo sentido do deslocamento que se observa neste ponto de saída quando o mecanismo é submetido ao primeiro carregamento, pode-se dizer, com base na eq.(2.30), que a maximização da energia mútua implica na maximização do deslocamento do ponto de saída do mecanismo. O terceiro carregamento deve ser considerado para o cálculo da flexibilidade média a ser minimizada. Assim, Nishiwaki et al. (1998), com base nestes três casos de carregamento, onde cada um dos quais é imposto sobre o domínio fixo de projeto, utilizaram a razão entre a energia mútua e a flexibilidade média como a função multi-objetivo para o projeto de mecanismos flexíveis. Percebe-se que, ao maximizar 
esta razão, maximiza-se a energia mútua do sistema e, ao mesmo tempo, minimiza-se a sua flexibilidade média - maximização da rigidez do mecanismo. Isso tudo, dentro das possibilidades que a relação de compromisso existente entre estas duas grandezas permite. A função multi-objetivo proposta por Nishiwaki et al. (1998), apesar de apresentar bons resultados, não permite que o projetista possa arbitrar sobre o valor da flexibilidade média do mecanismo, o que também não pode ser feito pela otimização de uma possível função multiobjetivo baseada na soma ponderada da energia mútua com a flexibilidade média. Funções como estas (baseadas na soma ponderada de parcelas) fornecem uma série de soluções conhecidas como "ótimos" de Pareto. As soluções "ótimas" de Pareto são obtidas através da variação dos pesos atribuídos às grandezas envolvidas na função multi-objetivo, sendo que, para o caso específico do projeto de mecanismos flexíveis, muitas destas soluções não apresentam um significado físico, seja porque a energia mútua assume um valor infinito, ou, seja porque, mesmo sendo finito o seu valor, a topologia apresenta regiões desconectadas entre si, conforme mostra a Figura 2.12.

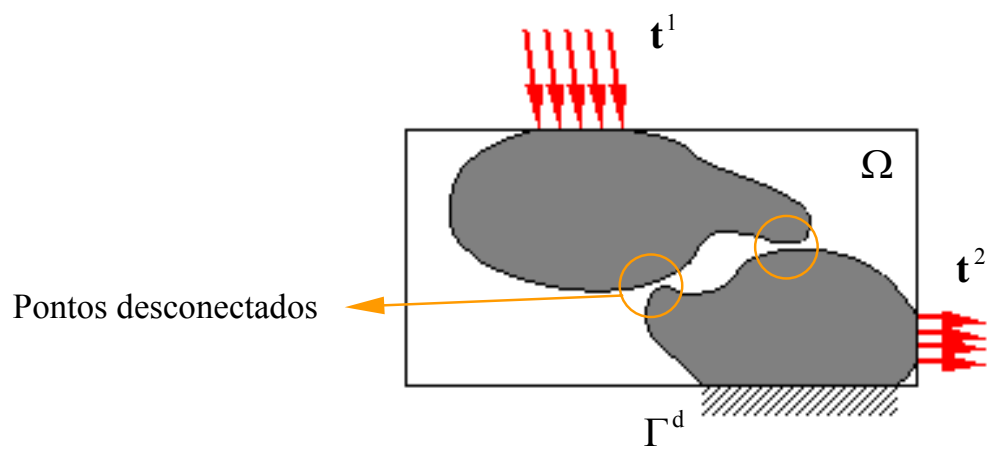

Figura 2.12 - Falta de conectividade entre duas regiões da topologia.

Para contornar esta questão das soluções degeneradas, Nishiwaki et al. (2001) propuseram então, uma função multi-objetivo na qual cada parcela corresponde à aplicação de uma função logarítmica à energia mútua e a flexibilidade média, respectivamente.

Com respeito às formulações para o projeto de mecanismos flexíveis, existem ainda aquelas que consideram a função multi-objetivo (compatibilizando a energia mútua e a flexibilidade média) conjuntamente com a mola acoplada ao ponto de saída do dispositivo (YIN; ANANTHASURESH, 2001; LOU et al., 2005). Nestes casos, a mola é considerada em apenas um dos dois carregamentos utilizados para determinar a energia mútua do mecanismo, ou seja, deve-se considerá-la exatamente naquele caso que corresponde ao carregamento externo para o qual o mecanismo flexível será submetido, uma vez que a rigidez da referida mola representa a peça de trabalho. 
Um outro aspecto que envolve o projeto de mecanismos flexíveis é a multiflexibilidade. Um exemplo que ilustra a multi-flexibilidade em estruturas mecânicas é o modo de deformação característico de uma carroceria de automóvel em função da aplicação de um carregamento em particular. Para obter estruturas "ótimas" que atendam a vários casos de flexibilidade, faz-se necessário estudá-los separadamente e compô-los num único problema de otimização. Cada modo de deformação apresentado pelo mecanismo é determinado conforme discutido anteriormente, ou seja, maximizando-se a energia mútua correspondente a dois carregamentos, bem como, minimizando-se a flexibilidade média obtida de um terceiro caso de carga aplicado sobre o domínio fixo de projeto. Em Nishiwaki et al. (2001), a função multi-objetivo capaz de compatibilizar todos os modos de deformação é uma extensão da função Kreisselmeier - Steinhauser (função-KS) (MLEJNEK; SCHIRRMACHER, 1993), com isso, devido à aplicação de funções logarítmicas e exponenciais, impõe-se a ocorrência de valores positivos às energias mútuas $^{5}$ relacionadas aos seus respectivos modos de deformação.

\subsection{EFEITO “DOBRADIÇA”}

A maioria dos mecanismos flexíveis obtidos por meio da otimização topológica apresenta articulações através das quais o mecanismo é capaz de fornecer deslocamentos maiores, ou forças mais intensas, dependendo da formulação empregada. Na literatura, estas articulações são conhecidas por one-node connected hinges. Neste trabalho, o fenômeno será denominado efeito "dobradiça", ou simplesmente, "dobradiça", que é caracterizada por dois elementos finitos sólidos conectados por um único nó, conforme mostra a Figura 2.13.

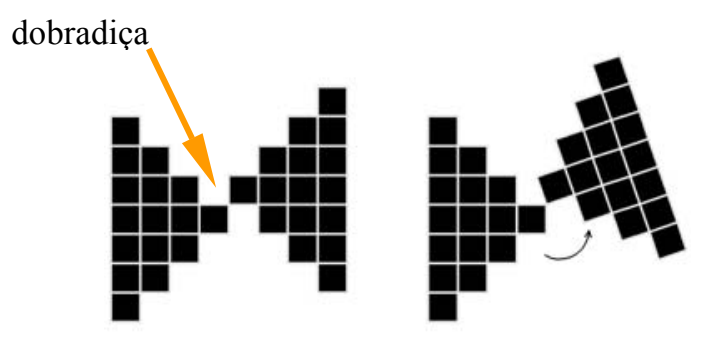

Figura 2.13 - Efeito dobradiça.

\footnotetext{
${ }^{5}$ Para que o deslocamento do ponto de saída do mecanismo flexível ocorra no sentido correto, é necessário que a energia mútua relacionada ao modo de deformação assuma valor positivo.
} 
Assim como no caso da instabilidade de tabuleiro, o efeito "dobradiça" é ocasionado por uma má modelagem numérica explorada pelo otimizador (BENDSØE; SIGMUND, 2003). As tensões e as deformações nestes pontos são inadequadamente modeladas por elementos finitos de ordem inferior (PEDERSEN; BUHL; SIGMUND, 2001), com isso, a "dobradiça" é entendida como a união de dois cantos artificialmente rígidos, de modo que, erroneamente, não surgem as tensões decorrentes da rotação relativa entre os elementos, caracterizando um movimento de corpo rígido entre eles. À luz destes dados, um balanço de energia pode explicar a ocorrência do problema. Considerando um meio contínuo discretizado em EF, sabe-se que ele terá grande capacidade de transferir trabalho mecânico - que é o objetivo das formulações discutidas na seção 2.4 -, desde que agregue pouca energia de deformação em seu interior. Para que isso seja levado ao extremo, a presença das "dobradiças" é um recurso matemático conveniente, pois, com a utilização de elementos finitos incapazes de reagir à aplicação dos momentos nos nós, o posicionamento estratégico destas "dobradiças" permitirá ao mecanismo gerar maiores deslocamentos, ou maiores forças de saída, sem que tanta energia seja absorvida. O uso de elementos finitos de ordem mais alta apenas alivia o problema (BENDSØE; SIGMUND, 2003), sendo necessário adotar restrições locais de tensão (DUYSINX; BENDSØE, 1998; BAHIA, 2005) como um meio mais efetivo para reduzir a ocorrência deste tipo de instabilidade, o que torna o problema de otimização custoso do ponto de vista computacional. Os métodos empregados para reduzir a instabilidade de tabuleiro e a dependência de malha se mostram ineficazes em relação às "dobradiças". A restrição de perímetro (HABER; JOG; BENDSØE, 1996) também não impede a sua ocorrência em determinados pontos da topologia. O método de controle dos gradientes das variáveis de projeto (PETERSSON; SIGMUND, 1998), bem como os filtros de densidade (BOURDIN, 2001), permitem o surgimento de "dobradiças" com densidades intermediárias. Pedersen; Buhl e Sigmund (2001) sugerem o pós-processamento da topologia como um meio de substituir as "dobradiças" por "pescoços", os quais são definidos pelo método da otimização de forma segundo Andersen (1997).
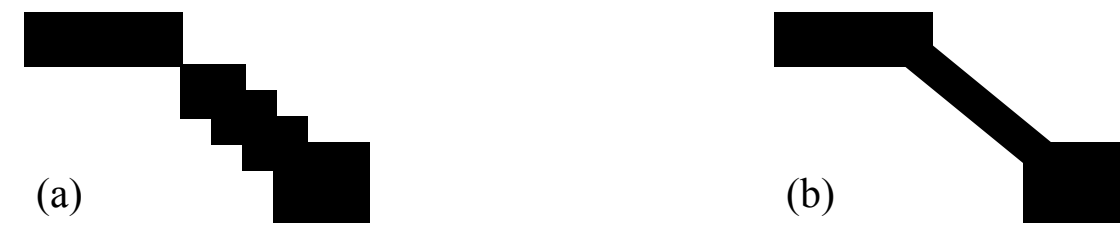

Figura 2.14 - (a) ocorrência do efeito dobradiça; (b) pescoço obtido por otimização de forma. 
O inconveniente desta abordagem está na redução do desempenho em relação ao que se pode esperar do mecanismo flexível original (antes do pós-processamento), pois a substituição das "dobradiças" pelos "pescoços" altera o valor da função objetivo definida no problema de otimização topológica. Poulsen (2001) propôs, já para o processo de otimização, um esquema que impõe uma largura mínima às regiões sólidas distribuídas pela topologia do mecanismo. O método é denominado MOnotonicity based minimum LEngth scale (MOLE) e consiste, basicamente, na introdução de uma restrição na formulação do problema de otimização topológica. Primeiramente é definido um funcional capaz de medir a "monotonicidade" da função densidade em cada ponto da topologia. Para tanto, num ponto a ser estudado, tem-se o centro de uma circunferência de diâmetro d. A "monotonicidade" da função correspondente à variável de projeto $f=\rho(\mathbf{x})$ é testada através do funcional (2.31) ao longo das quatro diagonais $t_{1}, t_{2}, t_{3} e_{4}$, igualmente espaçadas, todas contidas no interior da circunferência, vide Figura 2.15.

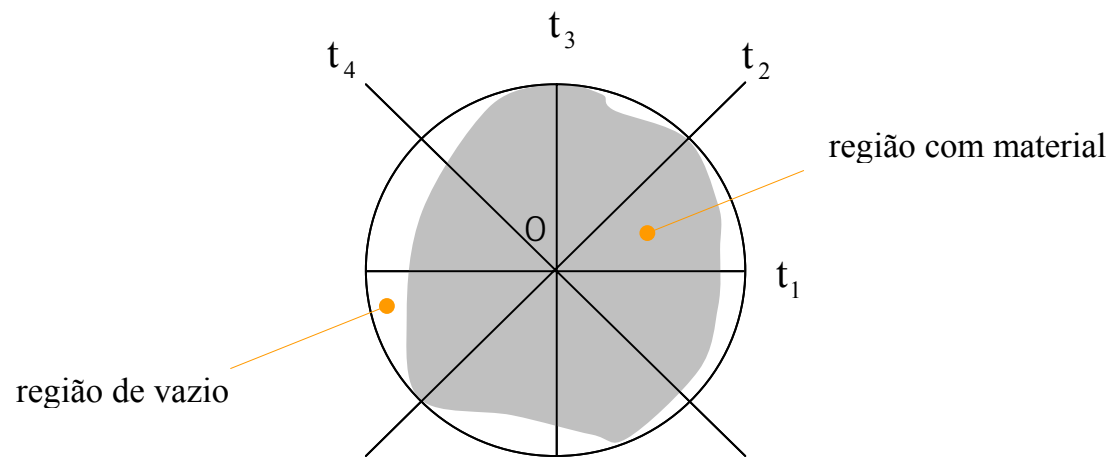

Figura 2.15 - Teste da "monotonicidade" na região delimitada pela circunferência de centro 0.

$$
\mathcal{M}_{\mathrm{d}, \mathrm{t}_{\mathrm{i}}}(\mathrm{f})=\int_{-\mathrm{d} / 2}^{\mathrm{d} / 2}\left|\mathrm{f}^{\prime}\left(\mathrm{t}_{\mathrm{i}}\right)\right| d \mathrm{t}_{\mathrm{i}}-\left|\int_{-\mathrm{d} / 2}^{\mathrm{d} / 2} \mathrm{f}^{\prime}\left(\mathrm{t}_{\mathrm{i}}\right) \mathrm{dt}_{\mathrm{i}}\right|
$$

A função f será considerada monotônica se, e somente se, $\mathcal{M}_{\mathrm{d}, \mathrm{t}_{\mathrm{i}}}(\mathrm{f})=0$. Neste caso, ao longo da diagonal $t_{i}$, para a qual o teste foi feito, $\rho$ apresentar-se-á constante, garantindo que a dimensão $\mathrm{d}$, sobre à diagonal testada, seja a largura mínima da topologia nesta região. Em qualquer outra situação, $\mathcal{M}_{\mathrm{d}, \mathrm{t}_{\mathrm{i}}}$ (f ) $>0$. Tomando como exemplo a Figura 2.15, a função que descreve o comportamento das densidades é monotônica apenas em relação à diagonal $t_{2}$. Para que o processo de otimização imponha a sobreposição de material em todas as diagonais 
de todas as circunferências da topologia, o que, em tese, eliminaria as "dobradiças", Poulsen propôs a seguinte restrição global na formulação do problema:

$$
\mathcal{L}(\mathrm{f}, \mathrm{d})=\int_{\Omega}\left[\sum_{\mathrm{i}=1}^{4} \mathcal{M}_{\mathrm{d}, \mathrm{t}_{\mathrm{i}}}(\mathrm{f})\right]^{\mathrm{p}} \mathrm{dx} \leq \delta
$$

onde $\delta$ é um número positivo relativamente pequeno e p é um fator de penalização, ambos arbitrários. Poulsen sugere ainda, que $\mathrm{p}$ assuma o valor de um número inteiro positivo e maior do que um, assim, pequenas variações da "monotonicidade", as quais poderiam contribuir para violar a restrição (2.32), são penalizadas a ponto de criar uma pequena dificuldade a esta violação, o que não altera a topologia do mecanismo.

Com intuíto de comparar os resultados obtidos em relação à redução das "dobradiças", Poulsen utilizou a mesma formulação que Sigmund (1997), contudo, substituiu o filtro de sensibilidades pela restrição MOLE, uma vez que o método proposto também tem por objetivo evitar a presença da instabilidade de tabuleiro, além de promover a independência de malha. O método MOLE gerou bons resultados em relação à redução das "dobradiças", no entanto, adaptar os funcionais (2.31) e (2.32) às versões discretizadas dos problemas de otimização se mostrou um tanto difícil. Como alternativa, o próprio Poulsen (2002) sugeriu um método simplificado para medir a "monotonicidade" da função densidade. Assim, considerando a discretização de um domínio fixo retangular em $m$ por $n$ elementos quadrados de quatro nós, para cada série de três elementos pertencentes a um grupo de quatro elementos - todos com um nó em comum -, calcula-se o valor de m tal como definido na eq.(2.33).

$$
\mathrm{m}(\mathrm{a}, \mathrm{b}, \mathrm{c})=|\mathrm{b}-\mathrm{a}|+|\mathrm{c}-\mathrm{b}|-|\mathrm{c}-\mathrm{a}|
$$

$\mathrm{Na}$ eq.(2.33), as variáveis a, b e c correspondem às densidades dos elementos cuja disposição é mostrada na Figura 2.16.

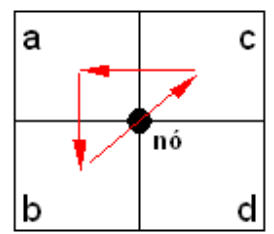

Figura 2.16 - Teste de "monotonicidade" para três elementos finitos de um grupo de quatro elementos. 
A seqüência de três elementos apresentará um comportamento monotônico para a função densidade se, e somente se, $\mathrm{m}(\mathrm{a}, \mathrm{b}, \mathrm{c})=0$, ou seja, $\mathrm{a} \geq \mathrm{b} \geq \mathrm{c}$, ou ainda, $\mathrm{a} \leq \mathrm{b} \leq \mathrm{c}$. Assim, como forma de considerar o comportamento da função densidade em todas as combinações de três elementos em seqüência, define-se:

$$
h(a, b, c, d)=m(a, b, c) \cdot m(a, c, d) \cdot m(b, a, c) \cdot m(b, d, c)
$$

se $h(a, b, c, d)=0$, então existe ao menos uma seqüência de três elementos que garante a "monotonicidade" da função densidade, e, portanto, o grupo de quatro elementos não formará uma "dobradiça". A Figura 2.17 traz possíveis valores de h, considerando que a densidade dos elementos escuros seja igual a um e a densidade dos elementos claros seja igual a zero.

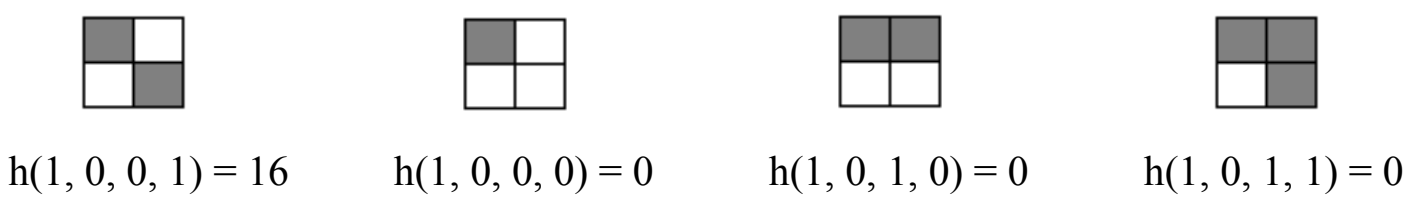

Figura 2.17 - Possíveis valores para h.

Poulsen (2002) utilizou as equações (2.33) e (2.34) como "caçadores" de “dobradiças", e, para forçar que estas fossem evitadas, introduziu a seguinte restrição global na formulação do problema de otimização topológica:

$$
H(\rho)=\sum_{j=1}^{m-1} \sum_{i=1}^{n-1} h\left(\rho_{i j}, \rho_{i+1 j}, \rho_{i j+1}, \rho_{i+1 j+1}\right) \leq \delta
$$

onde $\delta$ é um número arbitrário, positivo e relativamente pequeno.

Outros esquemas essencialmente matemáticos foram empregados no problema de otimização topológica como forma de evitar a presença das "dobradiças", dentre os quais se pode citar o tratamento multi-escalar do domínio discretizado (YOON et al., 2004) e o sistema de filtragem híbrida envolvendo as densidades e as sensibilidades (LUO et al., 2005).

Bahia (2005) elaborou uma formulação para projetar mecanismos flexíveis considerando os aspectos relacionados à fadiga. Para reduzir os custos computacionais decorrentes das restrições locais de tensão necessárias à sua formulação, o autor implementou um novo método, denominado patches nodais, através do qual cada uma destas restrições 
fica associada a uma grupo de elementos finitos e não a um elemento em particular. Desta forma, observaram-se bons resultados com respeito a redução das "dobradiças", que normalmente causam os picos de tensão mecânica nos mecanismos flexíveis.

Rahmatalla e Swan (2005) introduziram no problema de otimização dois carregamentos para o cálculo da energia mútua. Num destes carregamentos, uma mola na entrada e outra na saída do futuro mecanismo flexível são consideradas. A rigidez de ambas deverá ser equivalente à resistência que o domínio fixo de projeto (totalmente preenchido pelo material base) oferece quando submetido à carga aplicada no ponto de entrada, desde que haja um vínculo para o seu ponto de saída. Os autores demonstram, por meio dos resultados apresentados, que a maximização da energia mútua, relacionada aos dois carregamentos citados anteriormente, produz topologias livres de "dobradiças".

Com o intuito de reduzir a ocorrência do efeito "dobradiça" nas topologias dos mecanismos flexíveis, o código de projeto elaborado para este trabalho é baseado na formulação proposta por Cardoso e Fonseca (2004). Esta formulação não emprega restrições ou artifícios matemáticos que possam representar maiores dificuldades à sua implementação numérica. A essência da idéia está na própria função objetivo, que, diferentemente das abordagens discutidas acima, deve ser maximizada para armazenar a energia de deformação no interior do mecanismo. Com isso, o otimizador procura evitar a presença das "dobradiças", pois, desta forma, a função objetivo pode chegar aos valores mais extremos. Como se mostrará a seguir, as restrições dos deslocamentos na entrada e na saída do mecanismo têm seus gradientes calculados pelo método adjunto ${ }^{6}$. Estas restrições são necessárias para regular a quantidade de energia de deformação armazenada no interior do mecanismo projetado.

\footnotetext{
${ }^{6} \mathrm{O}$ método adjunto utilizado no cálculo das derivadas aproveita a forma fatorada da matriz de rigidez, reduzindo o custo computacional do processo de otimização. Os detalhes do método serão expostos na seção 2.7.
} 


\subsection{FORMULAÇÃO DO PROBLEMA DE OTIMIZAÇÃO TOPOLÓGICA}

\subsubsection{Comentários iniciais}

Um mecanismo flexível apresenta a capacidade de transferir força, movimento, ou mesmo provocar deformação em um corpo adjunto. Para tanto, ele deve possuir uma flexibilidade adequada à situação particular para a qual se destina. Fatores externos - dentre os quais: a força aplicada na entrada e a rigidez do corpo adjunto - influenciam na definição da sua topologia. A formulação usada neste trabalho (CARDOSO; FONSECA, 2004), baseada na maximização da energia de deformação, explora essa idéia de especificidade da aplicação. Para maximizar a energia no interior do mecanismo, faz-se necessário criar meios para que esta seja armazenada em regiões preenchidas pelo material base. Na seção 2.6.2, formula-se o problema de otimização com o qual é possível projetar os mecanismos flexíveis simples ${ }^{7}$. Na seção 2.6.3, o problema é estendido ao projeto de mecanismos multi-flexíveis. As versões numéricas para as formulações definidas nas seções 2.6 .2 e 2.6.3 são demonstradas nas seções 2.6.4 e 2.6.5, respectivamente.

\subsubsection{Formulação contínua do problema de otimização}

A parametrização do tensor constitutivo de material define as propriedades elásticas de cada ponto do domínio fixo em função da densidade pontual $(\rho)$ e do tensor de material base $\left(\mathbf{E}^{0}\right)$. Particularmente, na formulação empregada neste trabalho, utilizou-se a parametrização linear conforme a eq.(2.24), sendo $\rho \in[0,1]$. Para determinar a função objetivo, é importante saber que a energia de deformação específica de qualquer ponto submetido a um estado de tensões constante é inversamente proporcional à densidade pontual. Esta afirmação, sob certas condições, pode ser demonstrada segundo o raciocínio desenvolvido a seguir.

Suponha a existência de dois elementos infinitesimais, cada um dos quais submetidos ao mesmo estado de tensões. O primeiro é constituído de um material elástico linear cujo

\footnotetext{
${ }^{7}$ Neste texto, denominam-se mecanismos flexíveis simples aqueles mecanismos projetados para atender a um único carregamento.
} 
tensor é representado por $\mathbf{E}^{0}$. O segundo apresenta uma densidade $\rho$ em relação ao primeiro. Com a adoção da parametrização do tensor constitutivo para o segundo elemento, conforme a eq.(2.24), é possível escrever, segundo Cardoso e Fonseca (2004), a seguinte expressão:

$$
\boldsymbol{\sigma}=\mathbf{E}^{0} \boldsymbol{\varepsilon}^{0}=\left[\rho(\mathbf{x}) \mathbf{E}^{0}\right] \boldsymbol{\varepsilon} \Rightarrow \boldsymbol{\varepsilon}=\frac{1}{\rho(\mathbf{x})} \boldsymbol{\varepsilon}^{0}
$$

onde $\boldsymbol{\sigma}$ é o tensor representando o estado de tensões. Já $\boldsymbol{\varepsilon}^{0}$ e $\boldsymbol{\varepsilon}$ são, respectivamente, os tensores das deformações do primeiro e segundo elementos.

Escrevendo as energias de deformação específicas $\mathrm{w}^{0} \mathrm{e} \mathrm{w}$, respectivamente para cada um dos dois casos citados acima, tem-se para o primeiro deles:

$$
\mathrm{W}^{0}=\frac{1}{2} \boldsymbol{\varepsilon}^{0} \mathbf{E}^{0} \boldsymbol{\varepsilon}^{0}
$$

e para o segundo:

$$
\mathrm{W}=\frac{1}{2} \boldsymbol{\varepsilon}\left[\rho(\mathbf{x}) \mathbf{E}^{0}\right] \boldsymbol{\varepsilon}
$$

substituindo a eq.(2.36) na eq.(2.38), pode-se escrever:

$$
\mathrm{W}=\frac{1}{2}\left[\frac{1}{\rho(\mathbf{x})} \boldsymbol{\varepsilon}^{0}\right]\left[\rho(\mathbf{x}) \mathbf{E}^{0}\right]\left[\frac{1}{\rho(\mathbf{x})} \boldsymbol{\varepsilon}^{0}\right] \Rightarrow \mathrm{W}=\frac{1}{\rho(\mathbf{x})}\left[\frac{1}{2} \boldsymbol{\varepsilon}^{0} \mathbf{E}^{0} \boldsymbol{\varepsilon}^{0}\right]
$$

substituindo a eq.(2.37) na eq.(2.39), encontra-se finalmente a equação mostrada a seguir.

$$
\mathrm{w}=\frac{1}{\rho(\mathbf{x})} \mathrm{w}^{0}
$$

O gráfico da Figura 2.18 corresponde à eq.(2.40) e demonstra que a energia de deformação especifica (w) do segundo elemento infinitesimal se comporta de maneira inversamente proporcional à sua densidade $(\rho)$. 


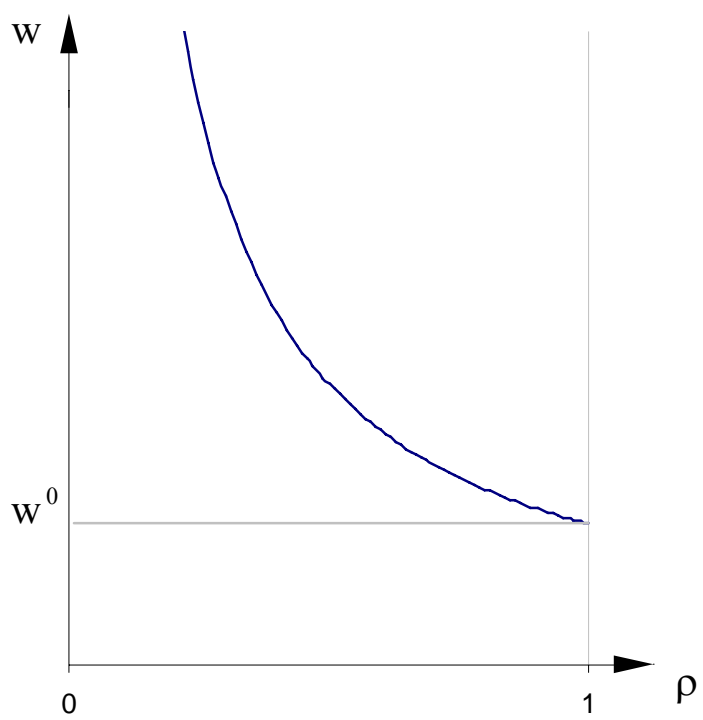

Figura 2.18 - Comportamento da energia de deformação específica.

Imagine agora um domínio fixo $\Omega$ sujeito a determinadas condições de contorno e a um carregamento externo t, conforme mostra a Figura 2.19.

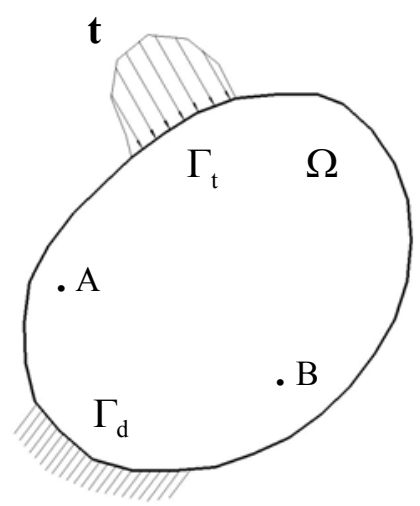

Figura 2.19 - Domínio fixo do problema de otimização submetido ao carregamento externo.

Uma função objetivo do problema correspondente ao projeto de mecanismos flexíveis pode ser definida ao se escrever a eq.(2.41), que corresponde à energia de deformação elástica (U) absorvida pelo domínio fixo em razão da aplicação do carregamento externo.

$$
\mathrm{U}=\int_{\Omega} \mathrm{W} \mathrm{d} \Omega
$$

Já que o objetivo é obter uma topologia que absorva o máximo de energia de deformação, distribui-se o material base de tal forma que o campo de densidades observado 
no interior de $\Omega$ maximize a eq.(2.41). Como a eq.(2.40) é aplicável a todos os pontos do domínio fixo, este deverá apresentar regiões de baixa densidade para que a maximização da eq.(2.41) ocorra. Do ponto de vista prático, esse tipo de resultado não tem utilidade, pois a formação do corpo do mecanismo flexível exige que a energia de deformação elástica seja armazenada em regiões cujos valores de densidade sejam iguais ou muito próximos a um. A alternativa para que isso ocorra é penalizar a energia de deformação específica de todos os pontos do domínio fixo $\Omega$. Isso pode ser feito multiplicando os termos da eq.(2.40) por uma potência da densidade, assim:

$$
\mathrm{w}^{*}=\left[\rho^{\mathrm{n}}(\mathbf{x})\right] \mathrm{w}=\left[\rho^{\mathrm{n}-1}(\mathbf{x})\right] \mathrm{w}^{0}
$$

onde $\mathrm{w}^{*}$ é justamente a energia de deformação especifica penalizada e $\mathrm{n}$ um número natural maior do que um. Esta última condição para n é a garantia de que a penalização de w ocorrerá nos casos onde os pontos tenham densidade inferior a um. Isso é mais facilmente entendido por meio do gráfico mostrado na Figura 2.20.

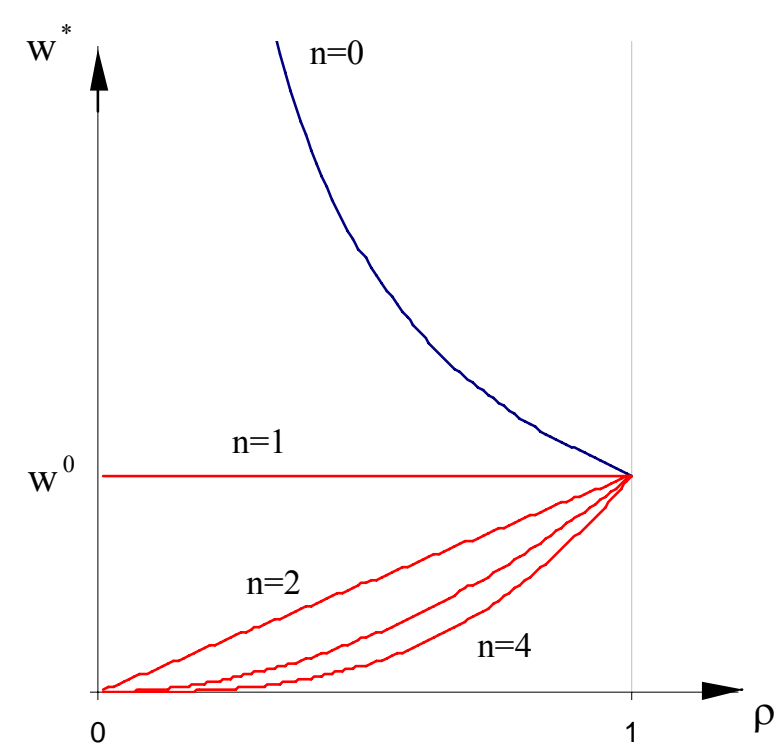

Figura 2.20 - Comportamento da energia de deformação específica penalizada.

Este gráfico corresponde à eq.(2.42) e nele se vê claramente que ${ }^{*}{ }^{*}$ estará compreendida entre zero e $\mathrm{w}^{0}$ quando os valores de $\mathrm{n}$ são superiores a um. Isso significa que a energia de deformação específica penalizada de um ponto qualquer do domínio fixo $\Omega$ será, no máximo, igual a sua energia de deformação específica correspondente à situação para a 
qual o referido ponto assume a densidade $(\rho)$ igual a um. Agora, considere $\phi$ como a integral da energia de deformação específica penalizada, tal como está escrito a seguir.

$$
\phi=\int_{\Omega} \mathrm{w}^{*} \mathrm{~d} \Omega \Rightarrow \phi=\int_{\Omega}\left[\rho^{\mathrm{n}}(\mathbf{x})\right] \mathrm{w} \mathrm{d} \Omega \quad \text { sendo } \quad \mathrm{n} \geq 2
$$

Aplicando a eq.(2.42) a todos os pontos do domínio fixo $\Omega$, este deverá apresentar regiões de alta densidade para que a eq.(2.43) seja maximizada. Assim, estar-se-á garantindo que a energia de deformação elástica se armazenará em regiões sólidas cujas propriedades elásticas sejam, se não as mesmas, no mínimo muito próximas daquelas conferidas pelo tensor constitutivo do material base $\left(\mathbf{E}^{0}\right)$. Para que a eq.(2.43) possa ser adotada como a função objetivo do problema de otimização, a quantidade de material do mecanismo flexível deve ser restringida, caso contrário, dada a necessidade de maximizar a referida equação, todo o domínio fixo $\Omega$ poderá ser preenchido pelo material base. Definindo $V_{\text {total }}$ como o volume máximo à topologia do mecanismo, escreve-se a restrição da forma mostrada abaixo.

$$
\int_{\Omega} \mathrm{d} \Omega-\mathrm{V}_{\text {total }} \leq 0
$$

Qualquer ponto de $\Omega$ é definido por três coordenadas espaciais, ou seja, $\mathbf{x}=\left\{\mathbf{x}_{1}, \mathbf{x}_{2}, \mathrm{x}_{3}\right\}^{\mathrm{T}}$. Com a aplicação do carregamento externo $\mathbf{t}$, o deslocamento dos pontos pertencentes ao domínio fixo pode ser escrito, em termos genéricos, da seguinte forma: $\mathbf{u}(\mathbf{x})=\left\{\mathrm{u}_{1}(\mathbf{x}), \mathrm{u}_{2}(\mathbf{x}), \mathrm{u}_{3}(\mathbf{x})\right\}^{\mathrm{T}}$. O comportamento cinemático do mecanismo será dado, então, em função das restrições de deslocamento impostas aos pontos de interesse pertencentes a $\Omega$. Assim, é possível definir o problema de otimização para o projeto de mecanismos flexíveis simples de acordo com a formulação (2.45). 


$$
\begin{array}{ll}
\text { Maximizar } & \phi=\int_{\Omega}\left[\rho^{\mathrm{n}}(\mathbf{x})\right] \mathrm{wd} \Omega \quad \text { sendo } \mathrm{n} \geq 2 \\
\text { Tal que: } \quad & \int_{\Gamma} \mathbf{t} \cdot \mathbf{u} \mathrm{d} \Gamma=\int_{\Omega} \boldsymbol{\varepsilon}(\mathbf{u}) \mathbf{E}(\mathbf{x}) \boldsymbol{\varepsilon}(\mathbf{v}) \mathrm{d} \Omega \quad \text { sendo } \quad \varepsilon_{\mathrm{ij}}(\mathbf{u})=\frac{1}{2}\left(\frac{\partial \mathrm{u}_{\mathrm{i}}}{\partial \mathrm{x}_{\mathrm{j}}}+\frac{\partial \mathrm{u}_{\mathrm{j}}}{\partial \mathbf{x}_{\mathrm{i}}}\right) \\
& \mathbf{E}(\mathbf{x})=\rho(\mathbf{x}) \mathbf{E}^{0}, \rho(\mathbf{x}) \in[0,1] \\
& \int_{\Omega} \mathrm{d} \Omega-\mathrm{V}_{\text {total }} \leq 0 \\
& \mathrm{u}_{\mathrm{i}}\left(\mathbf{x}_{\mathrm{A}}\right) \leq \mathrm{u}_{\mathrm{i} \text { max }}\left(\mathbf{x}_{\mathrm{A}}\right) \quad \text { sendo } \mathrm{i}=1,2 \text { ou } 3 \\
& \mathrm{u}_{\mathrm{j}}\left(\mathbf{x}_{\mathrm{B}}\right) \geq \mathrm{u}_{\mathrm{j} \min }\left(\mathbf{x}_{\mathrm{B}}\right) \quad \text { sendo } \mathrm{j}=1,2 \text { ou } 3
\end{array}
$$

Controle dos gradientes das variáveis de projeto.

A segunda equação da formulação (2.45) corresponde à condição de equilíbrio, nela foi desconsiderada a ação das forças de volume. Já o comportamento cinemático do mecanismo é definido, por exemplo, pela imposição de restrições aos deslocamentos dos pontos A e B pertencentes a $\Omega$. Outras restrições de deslocamento podem ser inseridas em função das necessidades do projeto.

A despeito da parametrização linear adotada para o tensor constitutivo de material, a penalização imposta à energia de deformação específica, com sua conseqüente influência sobre a função objetivo, confere à formulação características típicas de uma parametrização discreta do tensor de material, e, como discutido na seção 2.3.3, problemas dessa natureza não têm solução quando considerados sob a ótica da mecânica do contínuo. Para que o problema formulado apresente solução, deve-se impor o controle direto ou indireto dos gradientes das variáveis de projeto na distribuição de material efetuada ao longo do domínio fixo.

\subsubsection{Formulação contínua do problema de otimização aplicado à multi-flexibilidade}

Alguns mecanismos flexíveis devem ser projetados para desempenhar mais de uma tarefa, executando cada uma delas em função do carregamento aplicado - carregamentos distintos produzem formas distintas de deformação. Os dispositivos que atendem a estas condições são chamados de mecanismos multi-flexíveis. Para projetá-los através do MOT é necessário considerar todos os problemas de otimização num único, assim, a solução produzirá uma topologia capaz de atender às necessidades particulares de cada um deles. $\mathrm{O}$ 
objetivo desta seção é, portanto, estender a formulação contínua (2.45) ao projeto de mecanismos multi-flexíveis.

A Figura 2.21 representa um domínio fixo de projeto $\Omega$ submetido à c carregamentos, cada um dos quais aplicados em diferentes instantes de tempo.
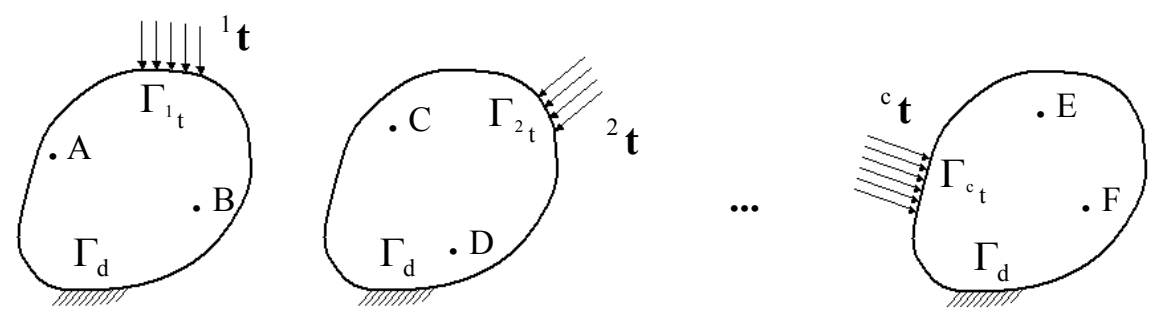

Figura 2.21 - Domínio fixo submetido a c carregamentos distintos.

Utilizando o que foi desenvolvido na seção 2.6.2, pode-se escrever a função objetivo de cada carregamento conforme a eq.(2.46).

$$
{ }^{\mathrm{q}} \phi=\int_{\Omega}\left[\rho^{\mathrm{n}}(\mathbf{x})\right]\left({ }^{\mathrm{q}} \mathrm{w}\right) \mathrm{d} \Omega \quad \text { sendo } \quad \mathrm{n} \geq 2 \text { e } \quad \mathrm{q}=1,2, \ldots, \mathrm{c}
$$

O projeto de um mecanismo multi-flexível capaz de atender às necessidades cinemáticas supostamente impostas aos pontos destacados nos carregamentos indicados pela Figura 2.21 implicará na eq.(2.47) como função multi-objetivo para o problema de otimização expresso em sua forma contínua.

$$
\phi_{\mathrm{MF}}=\sum_{\mathrm{q}=1}^{\mathrm{c}}{ }^{\mathrm{q}} \phi
$$

A formulação (2.48), escrita na seqüência, é completada com as equações de equilíbrio correspondentes aos seus respectivos casos de carregamento, com a restrição volumétrica e com as restrições de deslocamento, cada uma destas últimas relacionada apenas a um carregamento em particular. Assim, a formulação matemática do problema (2.48) empregado no projeto de mecanismos multi-flexíveis pode ser definida conforme se vê a seguir. 
$\operatorname{Max} \quad \phi_{\mathrm{MF}}=\sum_{\mathrm{q}=1}^{\mathrm{c}}{ }^{\mathrm{q}} \phi$

Tal que: $\quad \int_{\Gamma}^{1} \mathbf{t} \cdot{ }^{1} \mathbf{u} \mathrm{d} \Gamma=\int_{\Omega}^{1} \boldsymbol{\varepsilon}\left({ }^{1} \mathbf{u}\right) \mathbf{E}(\mathbf{x}){ }^{1} \boldsymbol{\varepsilon}\left({ }^{1} \mathbf{v}\right) \mathrm{d} \Omega \quad$ e $\quad{ }^{1} \varepsilon_{\mathrm{ij}}\left({ }^{1} \mathbf{u}\right)=\frac{1}{2}\left(\frac{\partial^{1} \mathbf{u}_{\mathrm{i}}}{\partial \mathrm{x}_{\mathrm{j}}}+\frac{\partial^{1} \mathbf{u}_{\mathrm{j}}}{\partial \mathrm{x}_{\mathrm{i}}}\right)$ $\int_{\Gamma}^{2} \mathbf{t} \cdot{ }^{2} \mathbf{u} \mathrm{d} \Gamma=\int_{\Omega}{ }^{2} \boldsymbol{\varepsilon}\left({ }^{2} \mathbf{u}\right) \mathbf{E}(\mathbf{x}){ }^{2} \boldsymbol{\varepsilon}\left({ }^{2} \mathbf{v}\right) \mathrm{d} \Omega \quad$ e $\quad{ }^{2} \varepsilon_{\mathrm{ij}}\left({ }^{2} \mathbf{u}\right)=\frac{1}{2}\left(\frac{\partial^{2} \mathrm{u}_{\mathrm{i}}}{\partial \mathrm{x}_{\mathrm{j}}}+\frac{\partial^{2} \mathrm{u}_{\mathrm{j}}}{\partial \mathrm{x}_{\mathrm{i}}}\right)$ $\int_{\Gamma}{ }^{\mathrm{c}} \mathbf{t} \cdot{ }^{\mathrm{c}} \mathbf{u} \mathrm{d} \Gamma=\int_{\Omega}{ }^{\mathrm{c}} \boldsymbol{\varepsilon}\left({ }^{\mathrm{c}} \mathbf{u}\right) \mathbf{E}(\mathbf{x}){ }^{\mathrm{c}} \boldsymbol{\varepsilon}\left({ }^{\mathrm{c}} \mathbf{v}\right) \mathrm{d} \Omega \quad$ e $\quad{ }^{\mathrm{c}} \varepsilon_{\mathrm{ij}}\left({ }^{\mathrm{c}} \mathbf{u}\right)=\frac{1}{2}\left(\frac{\partial^{\mathrm{c}} \mathrm{u}_{\mathrm{i}}}{\partial \mathrm{x}_{\mathrm{j}}}+\frac{\partial^{\mathrm{c}} \mathrm{u}_{\mathrm{j}}}{\partial \mathrm{x}_{\mathrm{i}}}\right)$ $\mathbf{E}(\mathbf{x})=\rho(\mathbf{x}) \mathbf{E}^{0}, \quad \rho(\mathbf{x}) \in[0,1]$ $\int_{\Omega} \mathrm{d} \Omega-\mathrm{V}_{\text {total }} \leq 0$

${ }^{1} \mathrm{u}_{\mathrm{i}}\left(\mathbf{x}_{\mathrm{A}}\right) \leq \mathrm{u}_{\mathrm{i} \max }\left(\mathbf{x}_{\mathrm{A}}\right)$ sendo $\mathrm{i}=1,2$ ou 3

${ }^{1} u_{j}\left(\mathbf{x}_{B}\right) \geq u_{j \min }\left(x_{B}\right)$ sendo $j=1,2$ ou 3

${ }^{2} \mathrm{u}_{\mathrm{i}}\left(\mathbf{x}_{\mathrm{C}}\right) \leq \mathrm{u}_{\mathrm{i} \max }\left(\mathbf{x}_{\mathrm{C}}\right)$ sendo $\mathrm{i}=1,2$ ou 3

${ }^{2} \mathrm{u}_{\mathrm{j}}\left(\mathbf{x}_{\mathrm{D}}\right) \geq \mathrm{u}_{\mathrm{jmin}}\left(\mathbf{x}_{\mathrm{D}}\right)$ sendo $\mathrm{j}=1,2$ ou 3

$\cdots$

$\cdots$

${ }^{\mathrm{c}} \mathrm{u}_{\mathrm{i}}\left(\mathbf{x}_{\mathrm{E}}\right) \leq \mathrm{u}_{\mathrm{imax}}\left(\mathbf{x}_{\mathrm{E}}\right)$ sendo $\mathrm{i}=1,2$ ou 3

${ }^{\mathrm{c}} \mathrm{u}_{\mathrm{j}}\left(\mathbf{x}_{\mathrm{F}}\right) \geq \mathrm{u}_{\mathrm{j} \min }\left(\mathbf{x}_{\mathrm{F}}\right)$ sendo $\mathrm{j}=1,2$ ou 3

Controle dos gradientes das variáveis de projeto.

Como no projeto de mecanismos flexíveis submetidos a um único carregamento, a solução do problema de otimização (2.48) requer o controle direto ou indireto dos gradientes das variáveis de projeto devido ao já discutido problema da dependência de malha.

Outro aspecto importante com respeito à formulação (2.48) está relacionado à não adoção de pesos para ponderar as parcelas da função multi-objetivo. Uma vez que os deslocamentos de entrada e saída são prescritos para cada carregamento, não haveria sentido impor tal ponderação, pois o comportamento cinemático imposto pelas restrições de deslocamento seria o mesmo independentemente dos valores atribuídos aos pesos. 


\subsubsection{Formulação numérica do problema de otimização}

Neste trabalho, a solução do problema de otimização combina o MEF com a programação linear seqüencial (PLS) (HAFTKA; GÜRDAL; KAMAT, 1990). Assim, a função objetivo definida pela eq.(2.43) - particularmente escrita para o projeto de mecanismos flexíveis submetidos a um único carregamento - deve ser ajustada para o domínio fixo $\Omega$ discretizado em EF, conforme mostra a equação escrita a seguir.

$$
\phi=\sum_{\mathrm{e}=1}^{\mathrm{N}} \int_{\mathrm{v}_{\mathrm{e}}} \rho^{\mathrm{n}}(\mathbf{x}) \mathrm{w}_{\mathrm{e}} \mathrm{dv}_{\mathrm{e}} \quad \text { sendo } \quad \mathrm{n} \geq 2
$$

$\mathrm{Na}$ eq.(2.49), cada uma das $\mathrm{N}$ parcelas do somatório está associada a um determinado elemento finito, para o qual $\mathrm{w}_{\mathrm{e}}$ determina a energia de deformação específica de um entre todos os pontos de seu volume $\mathrm{v}_{\mathrm{e}}$. Já a energia de deformação $\mathrm{U}_{\mathrm{e}}$, correspondente a um dado elemento em particular, pode ser escrita segundo a eq.(2.50).

$$
\mathrm{U}_{\mathrm{e}}=\int_{\mathrm{v}_{\mathrm{e}}} \mathrm{w}_{\mathrm{e}} \mathrm{dv} \mathrm{v}_{\mathrm{e}}
$$

Admitindo que a densidade $\rho_{\mathrm{e}}$ seja constante ao longo de todo volume $\mathrm{v}_{\mathrm{e}}$ do elemento finito considerado e substituindo a eq.(2.50) na eq.(2.49), pode-se escrever:

$$
\phi=\sum_{\mathrm{e}=1}^{\mathrm{N}} \rho_{\mathrm{e}}^{\mathrm{n}} \mathrm{U}_{\mathrm{e}} \quad \text { sendo } \quad \mathrm{n} \geq 2
$$

mas, substituindo a eq.(2.17) - decorrente da teoria do MEF - na eq.(2.51), tem-se uma nova forma de expressar $\phi$.

$$
\phi=\sum_{\mathrm{e}=1}^{\mathrm{N}} \rho_{\mathrm{e}}^{\mathrm{n}}\left(\frac{1}{2} \mathbf{u}_{\mathrm{e}}^{\mathrm{T}} \mathbf{k}_{\mathrm{e}} \mathbf{u}_{\mathrm{e}}\right) \Rightarrow \phi=\frac{1}{2} \sum_{\mathrm{e}=1}^{\mathrm{N}} \rho_{\mathrm{e}}^{\mathrm{n}} \mathbf{u}_{\mathrm{e}}^{\mathrm{T}} \mathbf{k}_{\mathrm{e}} \mathbf{u}_{\mathrm{e}} \quad \text { sendo } \mathrm{n} \geq 2
$$


Contudo, maximizar $\phi$ equivale a fazê-lo também para a expressão de seu valor duplicado $(\Phi=2 \phi)$. Logo, a favor da conveniência na implementação do algoritmo de otimização, adota-se a maximização de $\Phi$, conforme mostra a eq.(2.53).

$$
\Phi=\sum_{\mathrm{e}=1}^{\mathrm{N}} \rho_{\mathrm{e}}^{\mathrm{n}} \mathbf{u}_{\mathrm{e}}^{\mathrm{T}} \mathbf{k}_{\mathrm{e}} \mathbf{u}_{\mathrm{e}} \quad \text { sendo } \quad \mathrm{n} \geq 2
$$

Aqui, $\mathbf{k}_{\mathrm{e}}$ é a matriz de rigidez do elemento expressa através da eq.(2.18) - conforme demonstrado pela teoria do MEF (BATHE, 1996). Assim, aplicando a parametrização linear para a matriz de elasticidade $\mathbf{C}_{\mathrm{e}}$ presente na eq.(2.18), pode-se fazer:

$$
\mathbf{k}_{\mathrm{e}}=\int_{\mathrm{v}_{\mathrm{e}}} \mathbf{B}^{\mathrm{T}}\left(\rho_{\mathrm{e}} \mathbf{C}_{\mathrm{e}}^{0}\right) \mathbf{B} \mathrm{dv} \mathrm{v}_{\mathrm{e}} \Rightarrow \mathbf{k}_{\mathrm{e}}=\rho_{\mathrm{e}} \overbrace{\int_{\mathrm{v}_{\mathrm{e}}} \mathbf{B}^{\mathrm{T}} \mathbf{C}_{\mathrm{e}}^{0} \mathbf{B} \mathrm{d} \mathrm{v}_{\mathrm{e}}}^{\mathbf{k}_{\mathrm{e}}^{0}} \Rightarrow \mathbf{k}_{\mathrm{e}}=\rho_{\mathrm{e}} \mathbf{k}_{\mathrm{e}}^{0}
$$

onde $\mathbf{k}_{\mathrm{e}}^{0}$ e $\mathbf{C}_{\mathrm{e}}^{0}$ correspondem, respectivamente, as matrizes de rigidez e de elasticidade associadas à situação na qual o elemento finito é constituído de material base. Substituindo a eq.(2.54) na eq.(2.53), tem-se a função objetivo implementada no código para o projeto de mecanismo flexíveis submetidos a um único carregamento (mecanismos flexíveis simples).

$$
\Phi=\sum_{\mathrm{e}=1}^{\mathrm{N}} \rho_{\mathrm{e}}^{\mathrm{n}+1} \mathbf{u}_{\mathrm{e}}^{\mathrm{T}} \mathbf{k}_{\mathrm{e}}^{0} \mathbf{u}_{\mathrm{e}} \quad \text { sendo } \quad \mathrm{n} \geq 2
$$

Conforme dito anteriormente, para utilizar a eq.(2.55) como a função objetivo do problema de otimização é preciso adotar a restrição de volume, que aqui está representada em sua forma numérica pela inequação (2.56).

$$
\sum_{\mathrm{e}=1}^{\mathrm{N}} \rho_{\mathrm{e}} \mathrm{V}_{\mathrm{e}}-\mathrm{V}_{\text {total }} \leq 0
$$

Uma representação bastante simples da discretização de $\Omega$ pode ser vista na Figura 2.22 . 


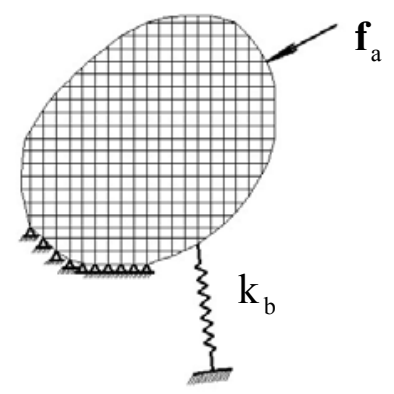

Figura 2.22 - Domínio fixo discretizado em $\mathrm{N}$ elementos finitos.

Todo grau de liberdade global associado a um nó que esteja sujeito a aplicação de uma força, ou que esteja ligado à rigidez de uma mola, deve apresentar uma restrição para limitar o deslocamento do nó em relação ao grau de liberdade considerado. Assim, este deslocamento ficará restrito a um valor absoluto máximo (limite máximo), caso o nó esteja sujeito à aplicação de uma força, e, caso o nó esteja acoplado a uma mola - cuja rigidez $\mathrm{k}_{\mathrm{b}}$ deva ser vencida pelo mecanismo flexível -, o deslocamento terá de assumir um valor absoluto mínimo (limite mínimo). Através destas restrições é possível regular os trabalhos externos atuantes no mecanismo flexível, o que influencia na quantidade de energia por ele absorvida.

As forças aplicadas e a rigidez das molas estarão alocadas na equação de equilíbrio (2.22), aplicável ao domínio fixo discretizado. As forças compõem o vetor $\mathbf{f}_{\mathrm{g}}$ e a rigidez das molas serão consideradas na matriz $\mathbf{k}_{\mathrm{g}}$, sendo que, cada rigidez acoplada a um grau de liberdade global será somada ao elemento da diagonal principal da matriz $\mathbf{k}_{\mathrm{g}}$ correspondente a este grau de liberdade. Com todo o exposto nesta seção, é possível estabelecer a formulação numérica do problema de otimização aplicado ao projeto de mecanismos flexíveis simples.

$$
\begin{array}{ll}
\text { Max } & \Phi=\sum_{\mathrm{e}=1}^{\mathrm{N}} \rho_{\mathrm{e}}^{\mathrm{n}+1} \mathbf{u}_{\mathrm{e}}^{\mathrm{T}} \mathbf{k}_{\mathrm{e}}^{0} \mathbf{u}_{\mathrm{e}} \quad \text { sendo } \mathrm{n} \geq 2 \quad \text { (função objetivo) } \\
\text { Tal que: } \quad & \mathbf{k}_{\mathrm{g}} \mathbf{u}_{\mathrm{g}}=\mathbf{f}_{\mathrm{g}} \quad \text { (equação de equilíbrio) } \\
& \sum_{\mathrm{e}=1}^{\mathrm{N}} \rho_{\mathrm{e}} \mathrm{v}_{\mathrm{e}}-\mathrm{V}_{\text {total }} \leq 0 \quad \text { (restrição de volume) } \\
& \left.\mathrm{u}_{\mathrm{g}_{\mathrm{i}}} \leq \mathrm{u}_{\mathrm{g}_{\mathrm{i}} \text { max }}, \ldots, \mathrm{u}_{\mathrm{g}_{\mathrm{k}}} \leq \mathrm{u}_{\mathrm{g}_{\mathrm{k}} \text { max }} \text { (restrições nos graus de liberdade globais } \mathrm{i}, \ldots, \mathrm{k}\right) \\
& \left.\mathrm{u}_{\mathrm{g}_{\mathrm{j}}} \geq \mathrm{u}_{\mathrm{g}_{\mathrm{j}} \min }, \ldots, \mathrm{u}_{\mathrm{g}_{\mathrm{l}}} \geq \mathrm{u}_{\mathrm{g}_{\mathrm{l}} \min } \quad \text { (restrições nos graus de liberdade globais } \mathrm{j}, \ldots, 1\right) \\
& \text { Limitar os gradientes das variáveis de projeto ou implementar filtro espacial. } \\
& 0,001 \leq \rho_{\mathrm{e}} \leq 1 \quad \text { sendo } \mathrm{e}=1,2,3, \ldots, \mathrm{N}
\end{array}
$$


A última restrição da formulação (2.57) limita a densidade dos elementos a um valor compreendido entre um número próximo de zero e um. Utiliza-se o limite mínimo diferente de zero, pois, caso contrário, haveria o risco de se criar uma singularidade na matriz de rigidez do domínio fixo $\Omega$. Neste trabalho, o valor adotado para o limite mínimo é de 0,001 (um milésimo), sendo este um valor adequado à maioria das aplicações do MOT.

A dependência do nível de discretização da malha é contornada neste trabalho com a utilização de um filtro espacial de densidades proposto por Cardoso e Fonseca (1999, 2003). Uma discussão mais ampla sobre as técnicas de filtragem poderá ser vista adiante, no capítulo 3, onde é abordada a implementação numérica do código computacional. Com a incorporação do filtro espacial de densidades à formulação (2.57) é possível gerar mecanismos flexíveis cujas topologias são menos sensíveis ao refinamento da malha.

É importante acrescentar que nem todos os problemas postos conforme a formulação (2.57) são solucionáveis, pois sempre haverá uma combinação de dados iniciais com os quais as topologias obtidas se apresentarão inadequadas. Entre tais dados iniciais, pode-se citar: volume disponível, geometria do domínio fixo, cargas aplicadas e restrições de deslocamento.

\subsubsection{Formulação numérica do problema de otimização aplicado à multi-flexibilidade}

Da mesma forma que na formulação utilizada para projetar os mecanismos flexíveis simples, a solução do problema de otimização relacionada à multi-flexibilidade se dá através da combinação entre o MEF e a PLS. Assim, faz-se necessário discretizar o domínio fixo de projeto $\Omega$ e elaborar a versão numérica da formulação (2.48). A Figura 2.23 representa os c carregamentos distintos aplicados a um domínio discretizado.
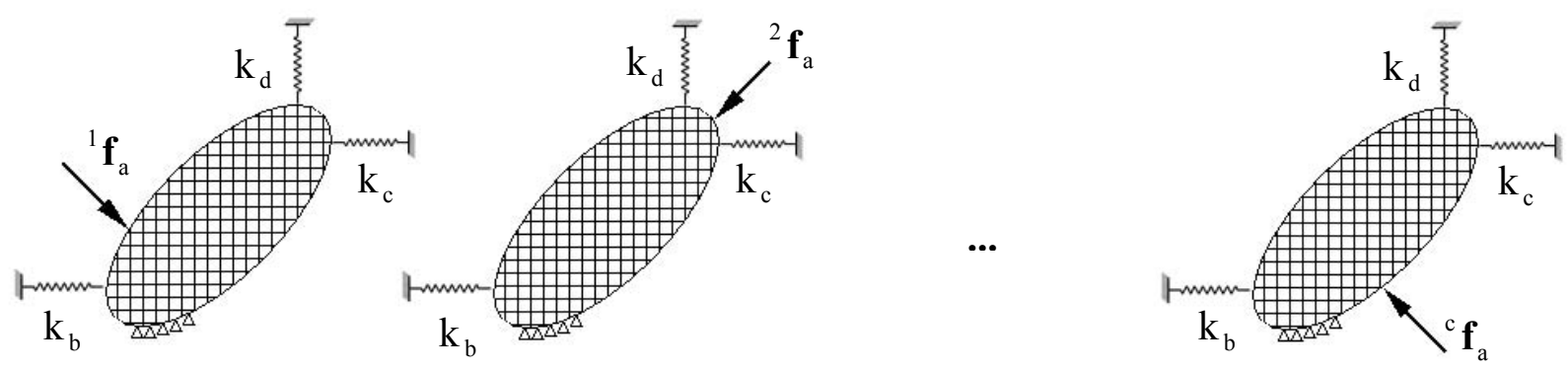

Figura 2.23 - Aplicação de c carregamentos sobre o domínio fixo de projeto discretizado em $\mathrm{N}$ elementos finitos. 
A versão da função multi-objetivo - análoga a eq.(2.47) - que corresponde ao problema numérico é expressa pela eq.(2.58).

$$
\Phi_{\mathrm{MF}}=\sum_{\mathrm{q}=1}^{\mathrm{c}}{ }^{\mathrm{q}} \Phi
$$

Cada uma das c parcelas ${ }^{\mathrm{q}} \Phi$ do somatório que define $\Phi_{\mathrm{MF}}$ está associada a um carregamento em particular. A eq.(2.55), cuja dedução foi apresentada na seção 2.6.4, é aplicável a estas parcelas. Assim, a eq.(2.58) pode ser expressa através da eq.(2.59).

$$
\Phi_{\mathrm{MF}}=\sum_{\mathrm{q}=1}^{\mathrm{c}} \sum_{\mathrm{e}=1}^{\mathrm{N}} \rho_{\mathrm{e}}^{\mathrm{n}+1}{ }^{\mathrm{q}} \mathbf{u}_{\mathrm{e}}^{\mathrm{T}} \mathbf{k}_{\mathrm{e}}^{0}{ }^{\mathrm{q}} \mathbf{u}_{\mathrm{e}} \quad \text { sendo } \quad \mathrm{n} \geq 2
$$

Adicionalmente a eq.(2.58), utilizada como função multi-objetivo, a formulação (2.60), equivalente ao problema (2.48), contém as versões numéricas para as equações de equilíbrio, para a restrição de volume e para as restrições dos deslocamentos restringidos.

$$
\begin{aligned}
& \operatorname{Max} \quad \Phi_{\mathrm{MF}}=\sum_{\mathrm{q}=1}^{\mathrm{c}}{ }^{\mathrm{q}} \Phi \quad \text { (função multi-objetivo) } \\
& \text { Tal que: } \quad \mathbf{k}_{\mathrm{g}}{ }^{1} \mathbf{u}_{\mathrm{g}}={ }^{1} \mathbf{f}_{\mathrm{g}} \quad \text { (equação de equilíbrio do } 1^{\circ} \text { carregamento) } \\
& \mathbf{k}_{\mathrm{g}}{ }^{\mathrm{c}} \mathbf{u}_{\mathrm{g}}={ }^{\mathrm{c}} \mathbf{f}_{\mathrm{g}} \quad \text { (equação de equilíbrio do "c-ésimo" carregamento) } \\
& \sum_{\mathrm{e}=1}^{\mathrm{N}} \rho_{\mathrm{e}} \mathrm{v}_{\mathrm{e}}-\mathrm{V}_{\text {total }} \leq 0 \quad \text { (restrição de volume) } \\
& { }^{1} \mathrm{u}_{\mathrm{g}_{\mathrm{i}}} \leq \mathrm{u}_{\mathrm{g}_{\mathrm{i}} \max }, \ldots,{ }^{1} \mathrm{u}_{\mathrm{g}_{\mathrm{k}}} \leq \mathrm{u}_{\mathrm{g}_{\mathrm{k}} \max } \text { (restrições nos graus de liberdade } \mathrm{i}, \ldots, \mathrm{k} \text { ) } \\
& { }^{1} u_{g_{j}} \geq u_{g_{j} \min }, \ldots,{ }^{1} u_{g_{1}} \geq u_{g_{l} \min } \quad \text { (restrições nos graus de liberdade } j, \ldots, l \text { ) } \\
& \text {... } \\
& \text {... } \\
& { }^{\mathrm{c}} \mathrm{u}_{\mathrm{g}_{\mathrm{q}}} \leq \mathrm{u}_{\mathrm{g}_{\mathrm{q}} \max }, \ldots,{ }^{\mathrm{c}} \mathrm{u}_{\mathrm{g}_{\mathrm{s}}} \leq \mathrm{u}_{\mathrm{g}_{\mathrm{s}} \max } \text { (restrições nos graus de liberdade } \mathrm{q}, \ldots, \mathrm{s} \text { ) } \\
& { }^{\mathrm{c}} \mathrm{u}_{\mathrm{g}_{\mathrm{r}}} \geq \mathrm{u}_{\mathrm{g}_{\mathrm{r}} \min }, \ldots,{ }^{\mathrm{c}} \mathrm{u}_{\mathrm{g}_{\mathrm{t}}} \geq \mathrm{u}_{\mathrm{g}_{\mathrm{t}} \min } \quad \text { (restrições nos graus de liberdade } \mathrm{r}, \ldots, \mathrm{t} \text { ) }
\end{aligned}
$$

Limitar os gradientes das variáveis de projeto ou implementar filtro espacial.

$$
0,001 \leq \rho_{\mathrm{e}} \leq 1 \quad \text { sendo } \mathrm{e}=1,2,3, \ldots, \mathrm{N}
$$


Assim como no projeto de mecanismos flexíveis simples, o filtro espacial de densidades (CARDOSO; FONSECA, 1999, 2003) foi incorporado à formulação (2.60).

\subsection{ANÁLISE DAS SENSIBILIDADES}

\subsubsection{Comentários iniciais}

As sensibilidades do problema de otimização são as derivadas da função objetivo e das demais funções restringidas, todas em relação às variáveis de projeto. $\mathrm{O}$ cálculo das sensibilidades é crucial, pois o método da PLS, empregado na solução do problema de OT, utiliza as primeiras derivadas destas funções para que elas sejam reescritas na forma linearizada. Uma explicação mais detalhada sobre esse processo é dada no capítulo que trata da implementação numérica do código computacional (capítulo 3). Neste trabalho, as sensibilidades foram calculadas pelo método adjunto. A cada iteração executada para o alcance da solução, o código computacional monta a matriz de rigidez global ( $\left.\mathbf{k}_{\mathrm{g}}\right)$ e a utiliza no cálculo dos deslocamentos $\mathbf{u}_{\mathrm{g}}$. Para tanto, $\mathbf{k}_{\mathrm{g}}$ é fatorada de modo a solucionar a eq.(2.22) eficazmente. Com isso, no método adjunto, a matriz $\mathbf{k}_{\mathrm{g}}$ fatorada é aproveitada no cálculo das sensibilidades, que é efetuado a cada iteração do processo de busca à topologia ótima. Nas formulações (2.57) e (2.60), não é necessário calcular as derivadas do volume restringido, uma vez que este já é uma função linear em relação às densidades. Nas seções 2.7.2 e 2.7.3, respectivamente, são desenvolvidas as sensibilidades da função objetivo e dos deslocamentos restringidos quando se deseja projetar mecanismos flexíveis submetidos a um único carregamento. Os gradientes das funções que integram o problema de projeto de mecanismos multi-flexíveis serão demonstrados na seção 2.7.4. 


\subsubsection{Sensibilidades da função objetivo}

A partir da eq.(2.53), equivalente à função objetivo da formulação (2.57), escreve-se:

$$
\Phi=\sum_{\mathrm{e}=1}^{\mathrm{N}}\left[\rho_{\mathrm{e}}^{\mathrm{n}}\left(\mathbf{H}_{\mathrm{e}} \mathbf{u}_{\mathrm{g}}\right)^{\mathrm{T}} \mathbf{k}_{\mathrm{e}}\left(\mathbf{H}_{\mathrm{e}} \mathbf{u}_{\mathrm{g}}\right)\right]+\boldsymbol{\lambda}_{\mathrm{g}}^{\mathrm{T}}\left(\mathbf{k}_{\mathrm{g}} \mathbf{u}_{\mathrm{g}}-\mathbf{f}_{\mathrm{g}}\right)
$$

onde $\mathbf{H}_{\mathrm{e}}$ é um operador matricial formado por elementos numéricos tais que seus valores sejam zero, ou um. Com a combinação destes dois números no interior da matriz do operador, este localizará, no vetor de deslocamentos globais $\left(\mathbf{u}_{\mathrm{g}}\right)$, os deslocamentos locais dos nós pertencentes ao elemento e. Já o vetor $\lambda_{g}$ corresponde a solução do problema adjunto, que consiste na imposição de um pseudo-carregamento ao domínio fixo $\Omega$. Dado que $\lambda_{\mathrm{g}}$ é multiplicado pelo vetor nulo advindo da equação de equilíbrio, a sua presença não altera o resultado apontado pela eq.(2.61) quando comparada à eq.(2.53). Derivando-se a eq.(2.61) com respeito à densidade $\rho_{\mathrm{i}}$ do "i-ésimo" elemento finito, tem-se:

$$
\begin{aligned}
& \frac{\partial \Phi}{\partial \rho_{\mathrm{i}}}=\sum_{\mathrm{e}=1}^{\mathrm{N}}\left[\frac{\partial \rho_{\mathrm{e}}^{\mathrm{n}}}{\partial \rho_{\mathrm{i}}}\left(\mathbf{H}_{\mathrm{e}} \mathbf{u}_{\mathrm{g}}\right)^{\mathrm{T}} \mathbf{k}_{\mathrm{e}}\left(\mathbf{H}_{\mathrm{e}} \mathbf{u}_{\mathrm{g}}\right)+2 \rho_{\mathrm{e}}^{\mathrm{n}}\left(\mathbf{H}_{\mathrm{e}} \mathbf{u}_{\mathrm{g}}\right)^{\mathrm{T}} \mathbf{k}_{\mathrm{e}} \mathbf{H}_{\mathrm{e}} \frac{\partial \mathbf{u}_{\mathrm{g}}}{\partial \rho_{\mathrm{i}}}+\rho_{\mathrm{e}}^{\mathrm{n}}\left(\mathbf{H}_{\mathrm{e}} \mathbf{u}_{\mathrm{g}}\right)^{\mathrm{T}} \frac{\partial \mathbf{k}_{\mathrm{e}}}{\partial \rho_{\mathrm{i}}}\left(\mathbf{H}_{\mathrm{e}} \mathbf{u}_{\mathrm{g}}\right)\right]+ \\
& +\boldsymbol{\lambda}_{\mathrm{g}}^{\mathrm{T}} \frac{\partial \mathbf{k}_{\mathrm{g}}}{\partial \rho_{\mathrm{i}}} \mathbf{u}_{\mathrm{g}}+\boldsymbol{\lambda}_{\mathrm{g}}^{\mathrm{T}} \mathbf{k}_{\mathrm{g}} \frac{\partial \mathbf{u}_{\mathrm{g}}}{\partial \rho_{\mathrm{i}}}
\end{aligned}
$$

onde a simetria da matriz de rigidez do elemento (e) contribui para simplificar a expressão do somatório. Agrupando os fatores comuns da eq.(2.62), chega-se à forma escrita a seguir.

$$
\begin{aligned}
& \frac{\partial \Phi}{\partial \rho_{\mathrm{i}}}=\overbrace{\sum_{\mathrm{e}=1}^{\mathrm{N}}\left[\frac{\partial \rho_{\mathrm{e}}^{\mathrm{n}}}{\partial \rho_{\mathrm{i}}}\left(\mathbf{H}_{\mathrm{e}} \mathbf{u}_{\mathrm{g}}\right)^{\mathrm{T}} \mathbf{k}_{\mathrm{e}}\left(\mathbf{H}_{\mathrm{e}} \mathbf{u}_{\mathrm{g}}\right)\right]}^{1^{\circ} \text { termo }}+\overbrace{\frac{\partial \mathbf{u}_{\mathrm{g}}}{\partial \rho_{\mathrm{i}}}\left[\sum_{\mathrm{e}=1}^{\mathrm{N}} 2 \rho_{\mathrm{e}}^{\mathrm{n}}\left(\mathbf{H}_{\mathrm{e}} \mathbf{u}_{\mathrm{g}}\right)^{\mathrm{T}} \mathbf{k}_{\mathrm{e}} \mathbf{H}_{\mathrm{e}}+\lambda_{\mathrm{g}}^{\mathrm{T}} \mathbf{k}_{\mathrm{g}}\right]}^{2^{\circ} \text { termo }}+ \\
& +\underbrace{\sum_{\mathrm{e}=1}^{\mathrm{N}} \rho_{\mathrm{e}}^{\mathrm{n}}\left(\mathbf{H}_{\mathrm{e}} \mathbf{u}_{\mathrm{g}}\right)^{\mathrm{T}} \frac{\partial \mathbf{k}_{\mathrm{e}}}{\partial \rho_{\mathrm{i}}}\left(\mathbf{H}_{\mathrm{e}} \mathbf{u}_{\mathrm{g}}\right)}_{3^{\circ} \text { termo }}+\underbrace{\lambda_{\mathrm{g}}^{\mathrm{T}} \frac{\partial \mathbf{k}_{\mathrm{g}}}{\partial \rho_{\mathrm{i}}} \mathbf{u}_{\mathrm{g}}}_{4^{\circ} \text { termo }}
\end{aligned}
$$


O primeiro termo da eq.(2.63) apresentará apenas uma parcela diferente de zero. Tratase daquela em que e $=\mathrm{i}$. Em todas as outras parcelas, a derivada da potência da densidade de um elemento, quando considerada em relação à densidade de outro elemento, será sempre nula. Portanto, este primeiro termo pode ser reescrito conforme está mostrado a seguir.

$$
\sum_{\mathrm{e}=1}^{\mathrm{N}}\left[\frac{\partial \rho_{\mathrm{e}}^{\mathrm{n}}}{\partial \rho_{\mathrm{i}}}\left(\mathbf{H}_{\mathrm{e}} \mathbf{u}_{\mathrm{g}}\right)^{\mathrm{T}} \mathbf{k}_{\mathrm{e}}\left(\mathbf{H}_{\mathrm{e}} \mathbf{u}_{\mathrm{g}}\right)\right]=\mathrm{n} \rho_{\mathrm{i}}^{\mathrm{n}-1} \mathbf{u}_{\mathrm{i}}^{\mathrm{T}} \mathbf{k}_{\mathrm{i}} \mathbf{u}_{\mathrm{i}}
$$

O segundo termo da eq.(2.63) deve ser anulado para evitar o cálculo das derivadas dos deslocamentos com respeito à densidade $\rho_{\mathrm{i}}$. Para tanto, faz-se:

$$
\sum_{\mathrm{e}=1}^{\mathrm{N}} 2 \rho_{\mathrm{e}}^{\mathrm{n}}\left(\mathbf{H}_{\mathrm{e}} \mathbf{u}_{\mathrm{g}}\right)^{\mathrm{T}} \mathbf{k}_{\mathrm{e}} \mathbf{H}_{\mathrm{e}}+\boldsymbol{\lambda}_{\mathrm{g}}^{\mathrm{T}} \mathbf{k}_{\mathrm{g}}=0 \Rightarrow \boldsymbol{\lambda}_{\mathrm{g}}^{\mathrm{T}} \mathbf{k}_{\mathrm{g}}=\sum_{\mathrm{e}=1}^{\mathrm{N}}-2 \rho_{\mathrm{e}}^{\mathrm{n}}\left(\mathbf{H}_{\mathrm{e}} \mathbf{u}_{\mathrm{g}}\right)^{\mathrm{T}} \mathbf{k}_{\mathrm{e}} \mathbf{H}_{\mathrm{e}}
$$

onde a eq.(2.65) corresponde ao problema adjunto (CARDOSO; FONSECA, 2004), dele se obtém o vetor $\lambda_{\mathrm{g}}$ através do MEF implementado no código computacional, o que não se constitui num esforço demasiado, pois a matriz $\mathbf{k}_{\mathrm{g}}$ já foi fatorada para a solução da equação de equilíbrio associada ao caso das cargas reais. O somatório no lado direito da eq.(2.65) é o pseudo-carregamento do problema.

O terceiro termo da eq.(2.63), assim como no primeiro termo da mesma, também apresentará apenas uma parcela diferente de zero, justamente àquela em que e = i . Em todas a outras parcelas, a derivada da matriz de rigidez de um elemento, quando considerada em relação à densidade de outro elemento, apresentar-se-á como uma matriz nula. Portanto, este terceiro termo é reescrito tal como mostrado abaixo.

$$
\sum_{\mathrm{e}=1}^{\mathrm{N}} \rho_{\mathrm{e}}^{\mathrm{n}}\left(\mathbf{H}_{\mathrm{e}} \mathbf{u}_{\mathrm{g}}\right)^{\mathrm{T}} \frac{\partial \mathbf{k}_{\mathrm{e}}}{\partial \rho_{\mathrm{i}}}\left(\mathbf{H}_{\mathrm{e}} \mathbf{u}_{\mathrm{g}}\right)=\rho_{\mathrm{i}}^{\mathrm{n}} \mathbf{u}_{\mathrm{i}}^{\mathrm{T}} \mathbf{k}_{\mathrm{i}}^{0} \mathbf{u}_{\mathrm{i}}
$$

No quarto termo da eq.(2.63), os elementos numéricos pertencentes a matriz $\mathbf{k}_{\mathrm{g}}$ e não dependentes da densidade $\rho_{\mathrm{i}}$ serão anulados no cálculo da derivada $\frac{\partial \mathbf{k}_{\mathrm{g}}}{\partial \rho_{\mathrm{i}}}$. Com isso, a matriz de rigidez $\mathbf{k}_{\mathrm{g}}$ e o vetor de deslocamentos $\mathbf{u}_{\mathrm{g}}$ podem ser substituídos por $\mathbf{k}_{\mathrm{i}}$ e $\mathbf{u}_{\mathrm{i}}$, 
respectivamente. Para adequar o tamanho do vetor $\lambda_{\mathrm{g}}$ e selecionar os seus elementos numéricos correspondentes ao "i-ésimo" elemento finito, pode-se multiplicá-lo pelo operador $\mathbf{H}_{\mathrm{i}}$ sem prejuízo ao resultado apontado por este quarto termo, o qual passará a ser escrito de acordo com a eq.(2.67).

$$
\boldsymbol{\lambda}_{\mathrm{g}}^{\mathrm{T}} \frac{\partial \mathbf{k}_{\mathrm{g}}}{\partial \rho_{\mathrm{i}}} \mathbf{u}_{\mathrm{g}}=\left(\mathbf{H}_{\mathrm{i}} \boldsymbol{\lambda}_{\mathrm{g}}\right)^{\mathrm{T}} \mathbf{k}_{\mathrm{i}}^{0} \mathbf{u}_{\mathrm{i}}
$$

Assim, somando-se o primeiro, o terceiro e o quarto termos, escritos conforme as equações (2.64), (2.66) e (2.67), respectivamente, tem-se a expressão final para o cálculo das derivadas da função objetivo em relação às variáveis de projeto, a qual deve ser escrita da seguinte forma:

$$
\frac{\partial \Phi}{\partial \rho_{\mathrm{i}}}=(\mathrm{n}+1) \rho_{\mathrm{i}}^{\mathrm{n}} \mathbf{u}_{\mathrm{i}}^{\mathrm{T}} \mathbf{k}_{\mathrm{i}}^{0} \mathbf{u}_{\mathrm{i}}+\left(\mathbf{H}_{\mathrm{i}} \boldsymbol{\lambda}_{\mathrm{g}}\right)^{\mathrm{T}} \mathbf{k}_{\mathrm{i}}^{0} \mathbf{u}_{\mathrm{i}}
$$

onde $\lambda_{\mathrm{g}}$ é a solução do problema adjunto representado pela eq.(2.65).

\subsubsection{Sensibilidades dos deslocamentos restringidos}

No cálculo das sensibilidades do deslocamento restringido correspondente ao " $\mathrm{j}$ ésimo" grau de liberdade global, também se faz uso da abordagem que envolve um problema adjunto, assim, inicialmente se escreve a equação:

$$
\mathrm{u}_{\mathrm{g}_{\mathrm{j}}}=\mathbf{L}_{\mathrm{j}}^{\mathrm{T}} \mathbf{u}_{\mathrm{g}}+\boldsymbol{\gamma}_{\mathrm{g}}^{\mathrm{T}}\left(\mathbf{k}_{\mathrm{g}} \mathbf{u}_{\mathrm{g}}-\mathbf{f}_{\mathrm{g}}\right)
$$

onde $\mathbf{L}_{\mathrm{j}}$ é um operador vetorial que localiza, no vetor de deslocamentos globais $\left(\mathbf{u}_{\mathrm{g}}\right)$, o deslocamento correspondente ao "j-ésimo" grau de liberdade global. Já o vetor $\gamma_{\mathrm{g}}$ é a solução do problema adjunto.

Derivando a eq.(2.69) em relação à densidade do "i-ésimo" elemento finito, tem-se: 


$$
\frac{\partial \mathbf{u}_{\mathrm{g}_{\mathrm{j}}}}{\partial \rho_{\mathrm{i}}}=\mathbf{L}_{\mathrm{j}}^{\mathrm{T}} \frac{\partial \mathbf{u}_{\mathrm{g}}}{\partial \rho_{\mathrm{i}}}+\gamma_{\mathrm{g}}^{\mathrm{T}} \frac{\partial \mathbf{k}_{\mathrm{g}}}{\partial \rho_{\mathrm{i}}} \mathbf{u}_{\mathrm{g}}+\boldsymbol{\gamma}_{\mathrm{g}}^{\mathrm{T}} \mathbf{k}_{\mathrm{g}} \frac{\partial \mathbf{u}_{\mathrm{g}}}{\partial \rho_{\mathrm{i}}}
$$

agrupando os fatores comuns da eq.(2.70), pode-se escrever:

$$
\frac{\partial \mathbf{u}_{\mathrm{g}_{\mathrm{j}}}}{\partial \rho_{\mathrm{i}}}=\underbrace{\frac{\partial \mathbf{u}_{\mathrm{g}}}{\partial \rho_{\mathrm{i}}}\left(\mathbf{L}_{\mathrm{j}}^{\mathrm{T}}+\boldsymbol{\gamma}_{\mathrm{g}}^{\mathrm{T}} \mathbf{k}_{\mathrm{g}}\right)}_{1^{\circ} \text { termo }}+\underbrace{\boldsymbol{\gamma}_{\mathrm{g}}^{\mathrm{T}} \frac{\partial \mathbf{k}_{\mathrm{g}}}{\partial \rho_{\mathrm{i}}} \mathbf{u}_{\mathrm{g}}}_{2^{{ }^{\text {termo }}}}
$$

ao anular o primeiro termo da eq.(2.71), tem-se:

$$
\mathbf{L}_{\mathrm{j}}^{\mathrm{T}}+\boldsymbol{\gamma}_{\mathrm{g}}^{\mathrm{T}} \mathbf{k}_{\mathrm{g}}=0 \Rightarrow \boldsymbol{\gamma}_{\mathrm{g}}^{\mathrm{T}} \mathbf{k}_{\mathrm{g}}=-\mathbf{L}_{\mathrm{j}}^{\mathrm{T}}
$$

onde a eq.(2.72) é o problema adjunto do qual se obtém o vetor $\gamma_{\mathrm{g}}$. O lado direito da eq.(2.72) corresponde ao pseudo-carregamento do referido problema.

O mesmo raciocínio usado na eq.(2.67) é aplicado aqui para simplificar o segundo termo da eq.(2.71). Com isso, chega-se à expressão final para o cálculo das derivadas dos deslocamentos restringidos em relação às variáveis de projeto, a qual deve ser escrita da seguinte forma:

$$
\frac{\partial u_{\mathrm{g}_{\mathrm{j}}}}{\partial \rho_{\mathrm{i}}}=\boldsymbol{\gamma}_{\mathrm{g}}^{\mathrm{T}} \frac{\partial \mathbf{k}_{\mathrm{g}}}{\partial \rho_{\mathrm{i}}} \mathbf{u}_{\mathrm{g}} \Rightarrow \frac{\partial \mathrm{u}_{\mathrm{g}_{\mathrm{j}}}}{\partial \rho_{\mathrm{i}}}=\left(\mathbf{H}_{\mathrm{i}} \boldsymbol{\gamma}_{\mathrm{g}}\right)^{\mathrm{T}} \mathbf{k}_{\mathrm{i}}^{0} \mathbf{u}_{\mathrm{i}}
$$

onde $\gamma_{\mathrm{g}}$ é a solução do problema adjunto representado pela eq.(2.72).

\subsubsection{Análise das sensibilidades para o problema de multi-flexibilidade}

No problema de multi-flexibilidade, o cálculo das sensibilidades da função multiobjetivo e dos deslocamentos restringidos são, respectivamente, feitos através das equações (2.68) e (2.73). Inicialmente, a derivada da função multi-objetivo é calculada com respeito a uma dada variável de projeto através da equação a seguir. 


$$
\frac{\partial \Phi_{\mathrm{MF}}}{\partial \rho_{\mathrm{i}}}=\frac{\partial^{1} \Phi}{\partial \rho_{\mathrm{i}}}+\frac{\partial^{2} \Phi}{\partial \rho_{\mathrm{i}}}+\ldots+\frac{\partial^{\mathrm{c}} \Phi}{\partial \rho_{\mathrm{i}}}
$$

Aplicando então, a eq.(2.68) a cada parcela da eq.(2.74), tem-se:

$$
\frac{\partial \Phi_{\mathrm{MF}}}{\partial \rho_{\mathrm{i}}}=\sum_{\mathrm{q}=1}^{\mathrm{c}}\left\{(\mathrm{n}+1) \rho_{\mathrm{i}}^{\mathrm{n} \mathrm{q}} \mathbf{u}_{\mathrm{i}}^{\mathrm{T}} \mathbf{k}_{\mathrm{i}}^{0 \mathrm{q}} \mathbf{u}_{\mathrm{i}}+\left(\mathbf{H}_{\mathrm{i}}{ }^{\mathrm{q}} \boldsymbol{\lambda}_{\mathrm{g}}\right)^{\mathrm{T}} \mathbf{k}_{\mathrm{i}}{ }^{0 \mathrm{q}} \mathbf{u}_{\mathrm{i}}\right\}
$$

onde ${ }^{\mathrm{q}} \boldsymbol{\lambda}_{\mathrm{g}}$ é a solução do problema adjunto correspondente ao "q-ésimo" carregamento. $\mathrm{O}$ problema adjunto, na sua forma genérica, pode ser representado pela eq.(2.76).

$$
{ }^{\mathrm{q}} \lambda_{\mathrm{g}}^{\mathrm{T}} \mathbf{k}_{\mathrm{g}}=\sum_{\mathrm{e}=1}^{\mathrm{N}}-2 \rho_{\mathrm{e}}^{\mathrm{n}}\left(\mathbf{H}_{\mathrm{e}}{ }^{\mathrm{q}} \mathbf{u}_{\mathrm{g}}\right)^{\mathrm{T}} \mathbf{k}_{\mathrm{e}} \mathbf{H}_{\mathrm{e}} \quad \text { sendo } \quad \mathrm{q}=1,2, \ldots, \mathrm{c}
$$

A derivada de um deslocamento restringido, definido para o "q-ésimo" carregamento, é calculada com respeito a uma dada variável de projeto através da aplicação direta da eq.(2.73). De forma geral, pode-se escrever:

$$
\frac{\partial^{\mathrm{q}} \mathrm{u}_{\mathrm{g}_{\mathrm{j}}}}{\partial \rho_{\mathrm{i}}}=\left(\mathbf{H}_{\mathrm{i}}{ }^{\mathrm{q}} \boldsymbol{\gamma}_{\mathrm{g}}\right)^{\mathrm{T}} \mathbf{k}_{\mathrm{i}}{ }^{0 \mathrm{q}} \mathbf{u}_{\mathrm{i}} \quad \text { sendo } \quad \mathrm{q}=1,2, \ldots, \mathrm{c}
$$

onde ${ }^{\mathrm{q}} \boldsymbol{\gamma}_{\mathrm{g}}$ é a solução do problema adjunto, que, escrito em termos genéricos, pode ser representado pela eq.(2.78).

$$
{ }^{\mathrm{q}} \boldsymbol{\gamma}_{\mathrm{g}}^{\mathrm{T}} \mathbf{k}_{\mathrm{g}}=-{ }^{\mathrm{q}} \mathbf{L}_{\mathrm{j}}^{\mathrm{T}} \quad \text { sendo } \quad \mathrm{q}=1,2, \ldots, \mathrm{c}
$$




\section{IMPLEMENTAÇÃO NUMÉRICA}

\subsection{INTRODUÇÃO}

Os problemas de otimização estrutural podem ser solucionados de duas formas. A primeira, e mais específica, é baseada em algum tipo de critério de optimalidade - optimality criteria - (ROZVANY, 1989). A determinação do critério dependerá do objetivo e da formulação do problema proposto. Assim, pode-se citar, como exemplo, o critério de optimalidade pelo qual uma topologia de maior rigidez é obtida a partir de uma quantidade restrita de material (SUZUKI; KIKUCHI, 1991; SIGMUND, 2001a). A maximização da freqüência de ressonância (MA; KIKUCHI; CHENG, 1995) e a otimização de estruturas discretas (ROZVANY; ZHOU, 1991) são exemplos de problemas solucionados com base no critério de optimalidade. Nestes casos, um algoritmo que busca a solução "ótima" é desenvolvido de forma semi-empírica para cada critério de optimalidade em particular, o que torna o processo de solução específico para a formulação do problema de otimização estrutural em questão. Esta especificidade confere ao método um bom desempenho computacional. A segunda forma de solucionar os problemas de otimização estrutural engloba os algoritmos fundamentados nos métodos de programação matemática, entre os quais podemos citar a programação linear (PL) (YANG; CHUANG, 1994) e os demais métodos não-lineares. A programação linear tem um papel importante em várias áreas além da Engenharia, uma vez que muitos problemas de otimização, relacionados a vários fenômenos quantificáveis, podem ser formulados através de funções exclusivamente lineares. O método SIMPLEX (DANTZIG, 1963) e o algoritmo de Karmarkar (1984) estão entre os meios mais comumente empregados na solução destes problemas lineares.

Uma forma muito útil para solucionar os problemas de otimização topológica nãolineares (quando ao menos uma das funções da formulação não é linear) é através da aplicação de algum método de aproximação, entre os quais podemos citar o método das assíntotas móveis - method of moving asymptotes (MMA) - (SVANBERG, 1987) e o método da programação linear seqüencial (HAFTKA; GÜRDAL; KAMAT, 1990). Ambos são muito usados em OT, pois são bem genéricos e de fácil adequação a qualquer problema de otimização estrutural. 
No MMA, faz-se a construção de subproblemas convexos, cada um deles pode ser entendido como uma aproximação em relação ao problema original, no entanto, tal aproximação é válida apenas entre os limites determinados pelas assíntotas. A solução de cada subproblema fornece subsídios para a construção do próximo, assim, esse processo iterativo perdurará até que haja a convergência para a solução "ótima”. Já na PLS, os subproblemas são considerados problemas lineares (que também são convexos). Contudo, esta consideração é válida apenas dentro de limites definidos por valores máximo e mínimo em relação a cada variável de projeto. Um método de programação linear é empregado na solução de cada um dos subproblemas linearizados, assim, da mesma forma que no MMA, a solução de um subproblema fornece dados para a montagem do próximo.

Neste trabalho, os problemas de OT postos conforme as formulações (2.57) e (2.60) foram solucionados por meio da combinação entre o método dos elementos finitos (BATHE, 1996) e a PLS. Para tanto, na execução do código computacional elaborado, a solução dos subproblemas lineares foi obtida por uma rotina escrita em linguagem $\mathrm{C}$ denominada GLPK. Esta rotina pode ser adquirida gratuitamente através da rede mundial de computadores (Internet) no endereço http://directory.fsf.org/math/misc/glpk.html pertencente à Free Software Directory (FSD), entidade que cataloga softwares gratuitos e que é patrocinada pela Free Software Foundation (FSF) e pela United Nations Education, Scientific and Cultural Organization (Unesco). Ainda que tenha operado em plataforma Windows, o GLPK (rotina baseada no método SIMPLEX) foi capaz de lidar eficazmente com domínios fixos discretizados em até 4050 elementos finitos. Este bom desempenho se explica devido a sua estrutura de dados esparsa, além da equivalência entre as formas primal e dual que a rotina explora em relação à formulação do subproblema de otimização. Na próxima seção 3.2, faz-se uma explanação mais detalhada sobre o método da PLS e sua aplicação neste trabalho. $\mathrm{Na}$ seção 3.3, discute-se a implementação do filtro espacial de densidades e suas características. Finalmente, na seção 3.4, dar-se-á maiores detalhes acerca do código computacional implementado. 


\subsection{PROGRAMAÇÃO LINEAR SEQÜENCIAL (PLS)}

\subsubsection{Comentários iniciais}

Como dito anteriormente, os problemas solucionados através dos métodos de PL têm a função objetivo e as restrições na forma mostrada pela formulação hipotética a seguir.

$$
\begin{array}{ll}
\text { Max } & f(x)=a_{1} x_{1}+a_{2} x_{2}+\ldots a_{N} x_{N} \\
\text { Tal que: } & g_{i}^{1}=b_{i}^{1} x_{1}+b_{i}^{2} x_{2}+\ldots+b_{i}^{N} x_{N}=k_{i}^{1} \text { sendo } i=1,2, \ldots, m_{1} \\
& g_{i}^{2}=c_{i}^{1} x_{1}+c_{i}^{2} x_{2}+\ldots+c_{i}^{N} x_{N} \geq k_{i}^{2} \text { sendo } i=1,2, \ldots, m_{2} \\
& g_{i}^{3}=d_{i}^{1} x_{1}+d_{i}^{2} x_{2}+\ldots+d_{i}^{N} x_{N} \leq k_{i}^{3} \text { sendo } i=1,2, \ldots, m_{3}
\end{array}
$$

Os problemas (2.57) e (2.60), pelos quais se quer projetar os mecanismos flexíveis, não são lineares, ou seja, nem todas as suas funções estão escritas linearmente, diferindo bastante do aspecto da formulação (3.1). Logo, para que os referidos problemas sejam solucionados por algum método de PL, é necessário linearizar suas funções por meio da Série de Taylor, a qual deve ser escrita até as suas parcelas de primeiro grau. Essa operação demanda, inicialmente, o cálculo das derivadas de primeira ordem das funções não-lineares, o que, em relação aos problemas (2.57) e (2.60), já foi feito nas seções 2.7.2, 2.7 .3 e 2.7.4. As derivações são efetuadas sempre em relação às variáveis de projeto $\left(\rho_{\mathrm{i}}\right)$, e mais especificamente, com respeito a um ponto $\left(\rho_{1}^{\mathrm{k}}, \rho_{2}^{\mathrm{k}}, \ldots, \rho_{\mathrm{N}}^{\mathrm{k}}\right)^{1}$ em particular. Apenas nas vizinhanças deste ponto é que as funções linearizadas apresentam uma aproximação aceitável em relação às suas respectivas funções originais. Assim, para que possamos trabalhar com as expressões reescritas através da série de Taylor, é necessário acrescentar novas restrições ao problema, com elas, cada uma das $\mathrm{N}$ variáveis de projeto $\left(\rho_{\mathrm{i}}\right)$ ficam limitadas a valores máximo e mínimo de tal forma que a boa aproximação das funções lineares seja garantida. Estas novas restrições são chamadas de limites móveis (THOMAS; VANDERPLAATS; SHYY, 1992). Agora, o problema linearizado, ou subproblema linear, pode então ser solucionado através de algum método de PL. Particularmente neste trabalho, a solução destes

\footnotetext{
${ }^{1}$ Ponto obtido como solução do subproblema da iteração anterior (k).
} 
subproblemas fornece os valores das densidades dos elementos finitos, com os quais o MEF determinará um novo campo de deslocamentos $\left(\mathbf{u}_{\mathrm{g}}\right)$, que em ato contínuo será utilizado no cálculo dos novos gradientes das funções. Com isso, têm-se subsídios para a montagem de um próximo subproblema linear. Repare que, nesse processo que se torna iterativo, os intervalos limitantes dos subproblemas precisam se deslocar a cada passo, daí a denominação de limites móveis. Nas próximas seções, 3.2.2 e 3.2.3, onde serão mostradas, respectivamente, as linearizações da função objetivo e das funções dos deslocamentos restringidos, a idéia de limites móveis será mais bem ilustrada. Por fim, na seção 3.2.4, serão descritas as formulações dos subproblemas lineares relacionados ao projeto de mecanismos flexíveis simples e de mecanismos multi-flexíveis.

\subsubsection{Linearização das funções objetivo e multi-objetivo}

Linearização correspondente ao projeto de mecanismos flexíveis simples

Escreve-se a Série de Taylor até as suas parcelas de primeiro grau para linearizar a função objetivo (2.57). Isso pode ser feito da seguinte forma:

$$
\Phi_{\mathrm{L}}(\boldsymbol{\rho})=\Phi\left(\boldsymbol{\rho}^{\mathrm{k}}\right)+\left.\frac{\partial \Phi}{\partial \rho_{1}}\right|_{\rho_{1}^{k}}\left(\rho_{1}-\rho_{1}^{\mathrm{k}}\right)+\left.\frac{\partial \Phi}{\partial \rho_{2}}\right|_{\rho_{2}^{k}}\left(\rho_{2}-\rho_{2}^{\mathrm{k}}\right)+\ldots+\left.\frac{\partial \Phi}{\partial \rho_{\mathrm{N}}}\right|_{\rho_{\mathrm{N}}^{\mathrm{k}}}\left(\rho_{\mathrm{N}}-\rho_{\mathrm{N}}^{\mathrm{k}}\right)
$$

onde $\Phi_{\mathrm{L}}$ é a função objetivo linearizada já apresentando um erro desconsiderado em relação a $\Phi$. Desenvolvendo a eq.(3.2) de modo a separar os termos constantes dos termos dependentes de $\boldsymbol{\rho}$, tem-se:

$$
\begin{aligned}
& \Phi_{\mathrm{L}}(\boldsymbol{\rho})=\overbrace{\Phi\left(\boldsymbol{\rho}^{\mathrm{k}}\right)-\left.\frac{\partial \Phi}{\partial \rho_{1}}\right|_{\rho_{1}^{k}} \rho_{1}^{\mathrm{k}}-\left.\frac{\partial \Phi}{\partial \rho_{2}}\right|_{\rho_{2}^{k}} \rho_{2}^{\mathrm{k}}-\ldots-\left.\frac{\partial \Phi}{\partial \rho_{\mathrm{N}}}\right|_{\rho_{\mathrm{N}}^{k}} \rho_{\mathrm{N}}^{\mathrm{k}}}^{\text {termos constantes }}+ \\
& +\underbrace{\left.\frac{\partial \Phi}{\partial \rho_{1}}\right|_{\rho_{1}^{k}} \rho_{1}+\left.\frac{\partial \Phi}{\partial \rho_{2}}\right|_{\rho_{2}^{k}} \rho_{2}+\ldots+\left.\frac{\partial \Phi}{\partial \rho_{\mathrm{N}}}\right|_{\rho_{\mathrm{N}}^{k}} \rho_{\mathrm{N}}}_{\text {termos dependentes }}
\end{aligned}
$$


para a maximização de $\Phi_{\mathrm{L}}$, é conveniente suprimir os termos constantes da eq.(3.3). Assim, a função objetivo linearizada é escrita conforme a eq.(3.4).

$$
\Phi_{L}(\boldsymbol{\rho})=\left.\frac{\partial \Phi}{\partial \rho_{1}}\right|_{\rho_{1}^{k}} \rho_{1}+\left.\frac{\partial \Phi}{\partial \rho_{2}}\right|_{\rho_{2}^{k}} \rho_{2}+\ldots+\left.\frac{\partial \Phi}{\partial \rho_{N}}\right|_{\rho_{N}^{k}} \rho_{N}
$$

A idéia da PLS é aproximar o problema de otimização a sucessivos subproblemas lineares. No caso da formulação (2.57), tais subproblemas terão sempre como função objetivo a eq.(3.4). Cada uma de suas parcelas é o produto da derivada parcial da função objetivo original - com respeito a densidade do "i-ésimo" elemento finito - pela sua respectiva variável de projeto $\left(\rho_{\mathrm{i}}\right)$. Na seção 2.7 .2 foi desenvolvida a expressão através da qual é possível calcular essas derivadas num determinado ponto $\left(\rho_{1}^{\mathrm{k}}, \rho_{2}^{\mathrm{k}}, \ldots, \rho_{\mathrm{N}}^{\mathrm{k}}\right)$ em particular.

Se considerarmos um problema de otimização onde a função objetivo apresenta uma única variável, será mais simples a compreensão sobre o papel dos limites móveis na PLS. Considere a maximização da função $F(x)$, representada graficamente conforme mostra a Figura 3.1.

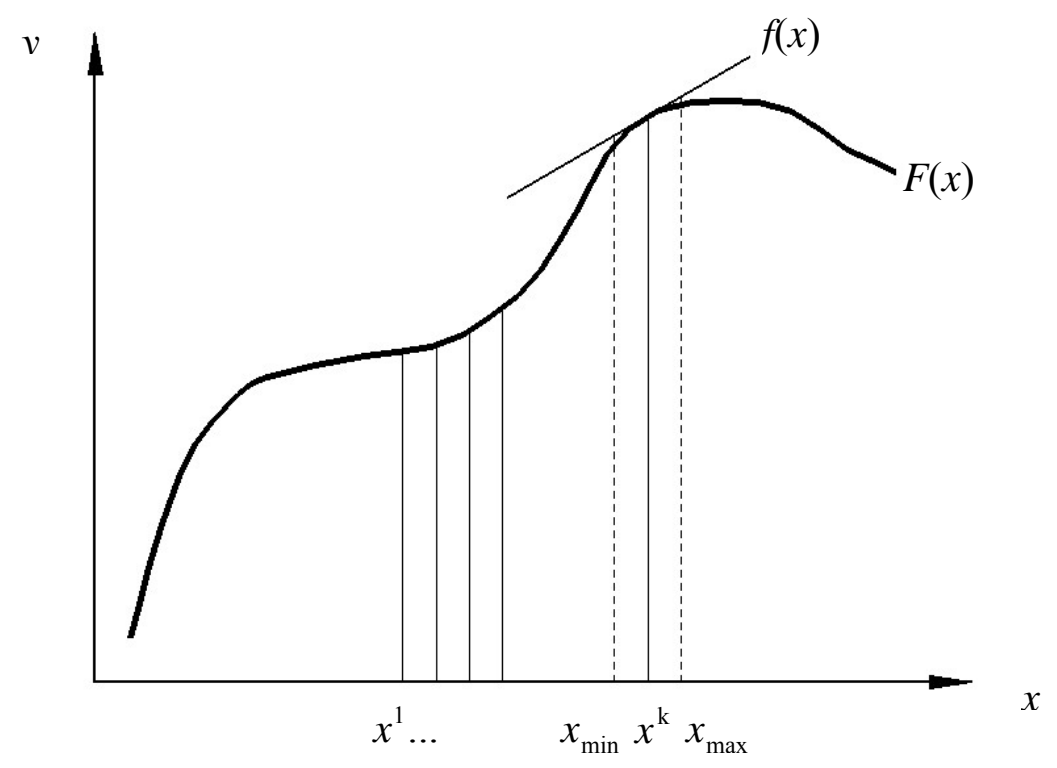

Figura 3.1 - Gráfico da função objetivo $F(x)$.

Observando a Figura 3.1 é possível notar que a função $F(x)$ foi linearizada no ponto correspondente à abscissa $x^{\mathrm{k}}$, que é obtida da solução do subproblema da iteração anterior k. 
Assim, por aproximação, $F(x)$ pode ser aceita como sendo a própria função $f(x)$, porém, isso é válido apenas na vizinhança delimitada por $x_{\min }$ e $x_{\max }$. O problema passa a ser, então, maximizar $f(x)$ no interior deste intervalo. É notório que a precisão do resultado dependerá do grau de proximidade destes limites em relação a $x^{\mathrm{k}}$. No caso particular da Figura 3.1, constata-se que o valor que maximiza $f(x)$ será exatamente $x_{\max }, \log 0, x^{\mathrm{k}+1}=x_{\max }$. Na seqüência, tem-se um novo subproblema que demandará a linearização de $F(x)$ no ponto correspondente à nova abscissa $x^{\mathrm{k}+1}$, bem como a necessidade de se adotar novos limites em torno de $x^{\mathrm{k}+1}$ - daí se justifica o termo limites móveis. Vai-se solucionando um subproblema após o outro até que se tenha a convergência de $x$ ao valor que maximize $F(x)$. A escolha dos limites móveis tem um papel importante na eficácia do processo de otimização, pois intervalos muito "apertados" implicam em processos mais demorados. Em contrapartida, intervalos mais "abertos" podem causar a perda do ponto "ótimo", ou seja, seria como se o valor de $x$ "passasse" pelo valor máximo de $F(x)$ sem que este último fosse detectado. Em termos práticos, neste caso, a função objetivo ficaria oscilando num campo de variação muito grande sem que houvesse a convergência à solução. Uma forma de contornar estes inconvenientes é controlar o tamanho do intervalo - definido por $x_{\min } \mathrm{e} x_{\max }$ - através da variação percentual entre dois valores consecutivos da função objetivo, o que pode ser feito com relativa simplicidade. Neste trabalho, dado o bom desempenho computacional da rotina GLPK, foi possível utilizar valores bem "apertados" para tais intervalos. Entre uma iteração e outra, para variações da função objetivo que se mostraram superiores a $60 \%$, utilizou-se $\rho_{\mathrm{i}_{\min }}=\rho_{\mathrm{i}}^{\mathrm{k}}-0,15 \rho_{\mathrm{i}}^{\mathrm{k}}$ como limites inferiores e $\rho_{\mathrm{i}_{\max }}=\rho_{\mathrm{i}}^{\mathrm{k}}+0,15 \rho_{\mathrm{i}}^{\mathrm{k}}$ como limites superiores. Para as variações entre $60 \%$ e $30 \%$, tem-se $\rho_{\mathrm{i}_{\min }}=\rho_{\mathrm{i}}^{\mathrm{k}}-0,10 \rho_{\mathrm{i}}^{\mathrm{k}}$ e $\rho_{\mathrm{i}_{\max }}=\rho_{\mathrm{i}}^{\mathrm{k}}+0,10 \rho_{\mathrm{i}}^{\mathrm{k}}$. E por fim, no caso de variações inferiores a $30 \%$, adotou-se $\rho_{i_{\min }}=\rho_{i}^{k}-0,05 \rho_{i}^{k}$ e $\rho_{i_{\max }}=\rho_{i}^{k}+0,05 \rho_{i}^{k}$.

Linearização correspondente ao projeto de mecanismos multi-flexíveis

A linearização da função multi-objetivo empregada no projeto de mecanismos multiflexíveis é uma extensão do procedimento demonstrado acima. Primeiramente, através do desenvolvimento da eq.(2.58), é possível escrever:

$$
\Phi_{\mathrm{MF}}={ }^{1} \Phi+{ }^{2} \Phi+\ldots+{ }^{\mathrm{c}} \Phi
$$


assim, de acordo com a eq.(3.4), a linearização da eq.(3.5) deve ser feita da seguinte forma:

$$
\Phi_{\mathrm{MF}_{\mathrm{L}}}(\boldsymbol{\rho})=\left.\frac{\partial\left({ }^{1} \Phi+{ }^{2} \Phi+\ldots+{ }^{\mathrm{c}} \Phi\right)}{\partial \rho_{1}}\right|_{\rho_{1}^{k}} \rho_{1}+\ldots+\left.\frac{\partial\left({ }^{1} \Phi+{ }^{2} \Phi+\ldots+{ }^{\mathrm{c}} \Phi\right)}{\partial \rho_{\mathrm{N}}}\right|_{\rho_{\mathrm{N}}^{\mathrm{k}}} \rho_{\mathrm{N}}
$$

reagrupando as parcelas da eq.(3.6), tem-se:

$$
\begin{aligned}
& \Phi_{\mathrm{MF}_{\mathrm{L}}}(\boldsymbol{\rho})=\left\{\left.\frac{\partial^{1} \Phi}{\partial \rho_{1}}\right|_{\rho_{1}^{k}} \rho_{1}+\ldots+\left.\frac{\partial^{1} \Phi}{\partial \rho_{\mathrm{N}}}\right|_{\rho_{\mathrm{N}}^{\mathrm{k}}} \rho_{\mathrm{N}}\right\}+\ldots+\left\{\left.\frac{\partial^{\mathrm{c}} \Phi}{\partial \rho_{1}}\right|_{\rho_{1}^{k}} \rho_{1}+\ldots+\left.\frac{\partial^{\mathrm{c}} \Phi}{\partial \rho_{\mathrm{N}}}\right|_{\rho_{\mathrm{N}}^{\mathrm{k}}} \rho_{\mathrm{N}}\right\} \Leftrightarrow \\
& \Phi_{\mathrm{MF}_{\mathrm{L}}}(\boldsymbol{\rho})=\sum_{\mathrm{q}=1}^{\mathrm{c}}{ }^{\mathrm{q}} \Phi_{\mathrm{L}}(\boldsymbol{\rho})
\end{aligned}
$$

onde $\Phi_{\mathrm{MF}_{\mathrm{L}}}$ corresponde à linearização da função multi-objetivo escrita por meio das parcelas ${ }^{\mathrm{q}} \Phi_{\mathrm{L}}$ linearizadas em relação ao ponto $\left(\rho_{1}^{\mathrm{k}}, \rho_{2}^{\mathrm{k}}, \ldots, \rho_{\mathrm{N}}^{\mathrm{k}}\right)$.

\subsubsection{Linearização das funções que representam os deslocamentos restringidos}

Neste trabalho, para aplicação do método da PLS, houve a necessidade de linearizar também as funções que representam os deslocamentos restringidos de alguns graus de liberdade globais. Da mesma forma que na linearização da função objetivo, utiliza-se a Série de Taylor escrita até as suas parcelas de primeiro grau. Assim, admitindo-se que para o “ $\mathrm{j}$ ésimo" grau de liberdade global haja um limite máximo de deslocamento, escreve-se:

$$
\begin{aligned}
& \mathrm{u}_{\mathrm{g}_{\mathrm{j}}} \leq \mathrm{u}_{\mathrm{g}_{\mathrm{j}} \max } \Rightarrow \\
& \mathrm{u}_{\mathrm{g}_{\mathrm{j}}}\left(\boldsymbol{\rho}^{\mathrm{k}}\right)+\left.\frac{\partial \mathrm{u}_{\mathrm{g}_{\mathrm{j}}}}{\partial \rho_{1}}\right|_{\rho_{1}^{\mathrm{k}}}\left(\rho_{1}-\rho_{1}^{\mathrm{k}}\right)+\left.\frac{\partial \mathrm{u}_{\mathrm{g}_{\mathrm{j}}}}{\partial \rho_{2}}\right|_{\rho_{2}^{\mathrm{k}}}\left(\rho_{2}-\rho_{2}^{\mathrm{k}}\right)+\ldots+\left.\frac{\partial \mathrm{u}_{\mathrm{g}_{\mathrm{j}}}}{\partial \rho_{\mathrm{N}}}\right|_{\rho_{\mathrm{N}}^{\mathrm{k}}}\left(\rho_{\mathrm{N}}-\rho_{\mathrm{N}}^{\mathrm{k}}\right) \leq \mathrm{u}_{\mathrm{g}_{\mathrm{j}} \max }
\end{aligned}
$$

desenvolvendo a inequação (3.8) de modo a separar os termos constantes dos termos dependentes de $\boldsymbol{\rho}$, tem-se a inequação escrita a seguir. 


$$
\begin{gathered}
\overbrace{u_{g_{j}}\left(\rho^{k}\right)-\left.\frac{\partial u_{g_{j}}}{\partial \rho_{1}}\right|_{\rho_{1}^{k}} \rho_{1}^{k}-\left.\frac{\partial u_{g_{j}}}{\partial \rho_{2}}\right|_{\rho_{2}^{k}} \rho_{2}^{k}-\ldots-\left.\frac{\partial u_{g_{j}}}{\partial \rho_{N}}\right|_{\rho_{N}^{k}} \rho_{N}^{k}}^{\text {termos constantes }}+ \\
+\underbrace{\left.\frac{\partial u_{g_{j}}}{\partial \rho_{1}}\right|_{\rho_{1}^{k}} \rho_{1}+\left.\frac{\partial u_{g_{j}}}{\partial \rho_{2}}\right|_{\rho_{2}^{k}} \rho_{2}+\ldots+\left.\frac{\partial u_{g_{j}}}{\partial \rho_{N}}\right|_{\rho_{N}^{k}} \rho_{N}}_{\text {termos dependentes }} \leq u_{g_{j} \max }
\end{gathered}
$$

Ao contrário do que foi feito com as funções objetivo e multi-objetivo linearizadas, na inequação (3.9) não se pode descartar os termos constantes, pois a solução do problema de otimização admite a maximização do deslocamento $u_{g_{j}}$ limitando-o, porém, a um valor máximo $u_{g_{j} \max }$.

Considerando uma função restringida que apresenta uma única variável, será mais simples entender o conceito acerca da linearização destas funções quando submetidas a tal condição.

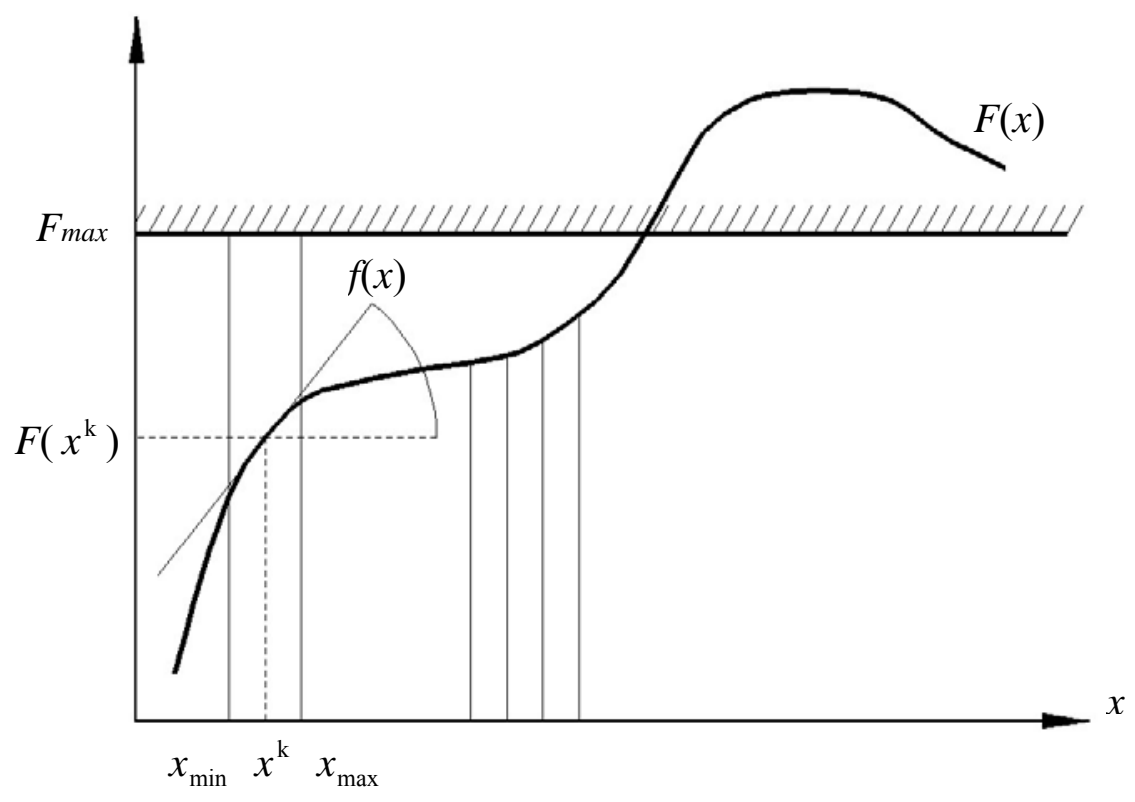

Figura 3.2 - Gráfico da função restringida $F(x)$.

Observando a Figura 3.2 é possível notar que, assim como no caso da função objetivo, a função restringida também está linearizada no ponto correspondente a abscissa $x^{\mathrm{k}}$, contudo, diferentemente do gráfico da Figura 3.1, o termo constante da função linearizada $f(x)$, do qual a ordenada $F\left(x^{\mathrm{k}}\right)$ é parcela integrante, não pode ser desprezado, caso contrário, perderia-se a 
referência na comparação de $f(x)$ com $F_{\max }$. Matematicamente, deve-se escrever: $f(x)=(d F / d x)_{x=x^{\mathrm{k}}} x+\left\{F\left(x^{\mathrm{k}}\right)-(d F / d x)_{x=x^{\mathrm{k}}} x\right\} \leq F_{\text {max }}$.

Assim, retornando a inequação (3.9), admite-se que:

$$
r_{j \max }=u_{g_{j}}\left(\rho^{k}\right)-\left.\frac{\partial u_{g_{j}}}{\partial \rho_{1}}\right|_{\rho_{1}^{k}} \rho_{1}^{k}-\left.\frac{\partial u_{g_{j}}}{\partial \rho_{2}}\right|_{\rho_{2}^{k}} \rho_{2}^{k}-\ldots-\left.\frac{\partial u_{g_{j}}}{\partial \rho_{N}}\right|_{\rho_{N}^{k}} \rho_{N}^{k}
$$

logo, substituindo a eq.(3.10) na inequação (3.9), tem-se:

$$
\left.\frac{\partial u_{g_{j}}}{\partial \rho_{1}}\right|_{\rho_{1}^{k}} \rho_{1}+\left.\frac{\partial u_{g_{j}}}{\partial \rho_{2}}\right|_{\rho_{2}^{k}} \rho_{2}+\ldots+\left.\frac{\partial u_{g_{j}}}{\partial \rho_{N}}\right|_{\rho_{N}^{k}} \rho_{N}+r_{j \max }-u_{g_{j} \max } \leq 0
$$

onde a inequação (3.11) representa a restrição de deslocamento do "j-ésimo" grau de liberdade global e está adequada à utilização do método da PLS. Cada uma de suas parcelas é o produto da derivada parcial do deslocamento $\mathrm{u}_{\mathrm{g}_{\mathrm{j}}}$ - com respeito a densidade do "i-ésimo" elemento finito - pela sua respectiva variável de projeto $\left(\rho_{\mathrm{i}}\right)$. Na seção 2.7.3 foi desenvolvida a expressão através da qual é possível calcular essas derivadas num determinado ponto $\left(\rho_{1}^{\mathrm{k}}, \rho_{2}^{\mathrm{k}}, \ldots, \rho_{\mathrm{N}}^{\mathrm{k}}\right)$ em particular.

Com um raciocínio análogo, chega-se a uma inequação similar para o caso onde o grau de liberdade global está limitado a um valor mínimo. Supondo tal condição ao "m-ésimo" grau de liberdade global, tem-se:

$$
\left.\frac{\partial u_{g_{m}}}{\partial \rho_{1}}\right|_{\rho_{1}^{k}} \rho_{1}+\left.\frac{\partial u_{g_{m}}}{\partial \rho_{2}}\right|_{\rho_{2}^{k}} \rho_{2}+\ldots+\left.\frac{\partial u_{g_{m}}}{\partial \rho_{N}}\right|_{\rho_{N}^{k}} \rho_{N}+r_{m \text { min }}-u_{g_{m} \min } \geq 0
$$

onde $r_{m \text { min }}$ e $u_{g_{m} \text { min }}$ correspondem a soma dos termos constantes da função linearizada e ao limite mínimo para o deslocamento do "m-ésimo” grau de liberdade global, respectivamente. 


\subsubsection{Formulação do subproblema linear}

Subproblema aplicado ao projeto de mecanismos flexíveis simples

A eq.(3.4) e as inequações (3.11) e (3.12) são linearizações efetuadas no ponto $\left(\rho_{1}^{\mathrm{k}}, \rho_{2}^{\mathrm{k}}, \ldots, \rho_{\mathrm{N}}^{\mathrm{k}}\right)$ correspondente à solução do subproblema da iteração anterior k. Assim, em termos genéricos, o subproblema de qualquer iteração pode ser escrito através da formulação mostrada abaixo.

$$
\begin{array}{ll}
\text { Max } \quad & \left.\frac{\partial \Phi}{\partial \rho_{1}}\right|_{\rho_{1}^{k}} \rho_{1}+\left.\frac{\partial \Phi}{\partial \rho_{2}}\right|_{\rho_{2}^{k}} \rho_{2}+\ldots+\left.\frac{\partial \Phi}{\partial \rho_{\mathrm{N}}}\right|_{\rho_{\mathrm{N}}^{k}} \rho_{\mathrm{N}} \\
\text { Tal que: } \quad & \sum_{\mathrm{e}=1}^{\mathrm{N}} \rho_{\mathrm{e}} \mathrm{v}_{\mathrm{e}}-\mathrm{V}_{\text {total }} \leq 0 \\
& \left.\frac{\partial \mathrm{u}_{\mathrm{g}_{\mathrm{j}}}}{\partial \rho_{1}}\right|_{\rho_{1}^{k}} \rho_{1}+\left.\frac{\partial \mathrm{u}_{\mathrm{g}_{\mathrm{j}}}}{\partial \rho_{2}}\right|_{\rho_{2}^{k}} \rho_{2}+\ldots+\left.\frac{\partial \mathrm{u}_{\mathrm{g}_{\mathrm{j}}}}{\partial \rho_{\mathrm{N}}}\right|_{\rho_{\mathrm{N}}^{k}} \rho_{\mathrm{N}}+\mathrm{r}_{\mathrm{jmax}}-\mathrm{u}_{\mathrm{g}_{\mathrm{j}} \max } \leq 0 \\
& \left.\frac{\partial \mathrm{u}_{\mathrm{g}_{\mathrm{m}}}}{\partial \rho_{1}}\right|_{\rho_{1}^{k}} \rho_{1}+\left.\frac{\partial \mathrm{u}_{\mathrm{g}_{\mathrm{m}}}}{\partial \rho_{2}}\right|_{\rho_{2}^{k}} \rho_{2}+\ldots+\left.\frac{\partial \mathrm{u}_{\mathrm{g}_{\mathrm{m}}}}{\partial \rho_{\mathrm{N}}}\right|_{\rho_{\mathrm{N}}^{k}} \rho_{\mathrm{N}}+\mathrm{r}_{\mathrm{m} \min }-\mathrm{u}_{\mathrm{g}_{\mathrm{m}} \min } \geq 0 \\
& 0,001 \leq \rho_{\mathrm{i}_{\min }} \leq \rho_{\mathrm{i}} \leq \rho_{\mathrm{i}_{\max }} \quad \text { sendo } \quad \mathrm{i}=1,2, \ldots, \mathrm{N}
\end{array}
$$

A formulação (3.13) é válida dentro do campo delimitado pelos limites móveis definidos na última restrição e, uma vez que todas as suas funções são lineares, o problema pode ser solucionado por algum método de PL. A condição de equilíbrio, não explícita no subproblema, decorre da utilização de um campo de deslocamentos que, assim como as densidades obtidas da iteração anterior, é usado na determinação dos gradientes das funções. Obtém-se tal campo de deslocamentos através da análise por MEF em relação ao domínio fixo discretizado.

Subproblema aplicado ao projeto de mecanismos multi-flexíveis

A formulação (3.14), descrita a seguir, corresponde ao subproblema de otimização aplicado ao projeto de mecanismos multi-flexíveis submetidos a c carregamentos distintos. 


$$
\begin{aligned}
& \operatorname{Max} \quad \Phi_{\mathrm{MF}_{\mathrm{L}}}(\boldsymbol{\rho})=\sum_{\mathrm{q}=1}^{\mathrm{c}}{ }^{\mathrm{q}} \Phi_{\mathrm{L}}(\boldsymbol{\rho}) \\
& \text { Tal que: } \quad \sum_{\mathrm{e}=1}^{\mathrm{N}} \rho_{\mathrm{e}} \mathrm{v}_{\mathrm{e}}-\mathrm{V}_{\text {total }} \leq 0 \\
& \left.\frac{\partial^{1} u_{g_{i}}}{\partial \rho_{1}}\right|_{\rho_{1}^{k}} \rho_{1}+\left.\frac{\partial^{1} u_{g_{i}}}{\partial \rho_{2}}\right|_{\rho_{2}^{k}} \rho_{2}+\ldots+\left.\frac{\partial^{1} u_{g_{i}}}{\partial \rho_{N}}\right|_{\rho_{\mathrm{N}}^{k}} \rho_{N}+{ }^{1} r_{i \max }-u_{g_{i} \max } \leq 0 \\
& \left.\frac{\partial^{1} u_{g_{j}}}{\partial \rho_{1}}\right|_{\rho_{1}^{k}} \rho_{1}+\left.\frac{\partial^{1} u_{g_{j}}}{\partial \rho_{2}}\right|_{\rho_{2}^{k}} \rho_{2}+\ldots+\left.\frac{\partial^{1} u_{g_{j}}}{\partial \rho_{N}}\right|_{\rho_{N}^{k}} \rho_{N}+{ }^{1} r_{j \text { min }}-u_{g_{j} \min } \geq 0 \\
& \left.\frac{\partial^{c} u_{g_{q}}}{\partial \rho_{1}}\right|_{\rho_{1}^{k}} \rho_{1}+\left.\frac{\partial^{c} u_{g_{q}}}{\partial \rho_{2}}\right|_{\rho_{2}^{k}} \rho_{2}+\ldots+\left.\frac{\partial^{c} u_{g_{q}}}{\partial \rho_{N}}\right|_{\rho_{N}^{k}} \rho_{N}+{ }^{c} r_{q} \max -u_{g_{q} \max } \leq 0 \\
& \left.\frac{\partial^{c} u_{g_{r}}}{\partial \rho_{1}}\right|_{\rho_{1}^{k}} \rho_{1}+\left.\frac{\partial^{c} u_{g_{r}}}{\partial \rho_{2}}\right|_{\rho_{2}^{k}} \rho_{2}+\ldots+\left.\frac{\partial^{c} u_{g_{r}}}{\partial \rho_{N}}\right|_{\rho_{N}^{k}} \rho_{N}+{ }^{c} r_{r \min }-u_{g_{r} \min } \geq 0 \\
& 0,001 \leq \rho_{\mathrm{i}_{\min }} \leq \rho_{\mathrm{i}} \leq \rho_{\mathrm{i}_{\max }} \quad \text { sendo } \mathrm{i}=1,2, \ldots, \mathrm{N}
\end{aligned}
$$

Além da eq.(3.7), empregada como função multi-objetivo, o subproblema traz ainda a restrição de volume e os deslocamentos restringidos linearizados em relação ao ponto $\left(\rho_{1}^{\mathrm{k}}, \rho_{2}^{\mathrm{k}}, \ldots, \rho_{\mathrm{N}}^{\mathrm{k}}\right)$. As condições de equilíbrio que foram impostas a cada um dos c carregamentos, da mesma forma que no subproblema (3.13), também não estão explícitas, contudo, os seus respectivos campos de deslocamento, obtidos através do MEF, são utilizados na determinação dos gradientes necessários à elaboração das equações que definem este subproblema (3.14). 


\subsection{IMPLEMENTAÇÃO DO FILTRO ESPACIAL DE DENSIDADES}

Na seção 2.3, um dos aspectos discutidos sobre o MOT diz respeito à dependência dos resultados obtidos em relação ao nível de discretização do domínio fixo inicial. Métodos de restrição, através dos quais se faz o controle da topologia obtida (HABER et al., 1996), são recursos utilizados para evitar esta dependência. Neste trabalho, o código computacional empregado no projeto de mecanismos flexíveis incorpora um filtro espacial de densidades que é baseado na idéia do raio de abrangência (CARDOSO; FONSECA, 1999; CARDOSO; FONSECA, 2003). Tal recurso numérico reduz a dependência dos resultados com respeito ao nível de discretização da malha, diferentemente do filtro de densidades por vizinhança fixa (SWAN; KOSAKA, 1997), que só se presta à redução do problema da instabilidade de tabuleiro. Neste último tipo de filtro, a densidade de cada elemento depende das densidades dos elementos vizinhos, sejam estes vizinhos de aresta ou de vértice, vide Figura 3.3.

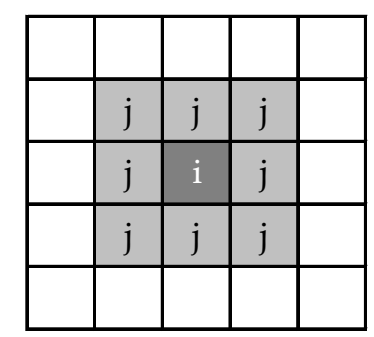

Figura 3.3 - Elemento central i sofrendo a influência dos elementos adjacentes.

A forma pela qual a densidade do elemento i sofre a influência das densidades dos demais elementos j é escrita por meio da eq.(3.15).

$$
\rho_{\mathrm{i}}^{\text {ajustado }}=\frac{\rho_{\mathrm{i}} \mathrm{V}_{\mathrm{i}}+w_{1} \sum_{\mathrm{j}} \rho_{\mathrm{j}} \mathrm{V}_{\mathrm{j}}+w_{2} \sum_{\mathrm{j}} \rho_{\mathrm{j}} \mathrm{V}_{\mathrm{j}}}{\mathrm{V}_{\mathrm{i}}+w_{1} \sum_{\mathrm{j}} \mathrm{V}_{\mathrm{j}}+w_{2} \sum_{\mathrm{j}} \mathrm{V}_{\mathrm{j}}}
$$

O termo $\rho_{\mathrm{i}}^{\text {ajustado }}$ é a densidade ajustada do elemento i, que inicialmente apresenta a densidade $\rho_{\mathrm{i}}$. Os valores $w_{1}$ e $w_{2}$ são pesos atribuídos, de forma heurística, aos elementos vizinhos de aresta e vértice, respectivamente. Nota-se que, se houver um suposto refinamento da malha inicialmente representada pela Figura 3.3, o filtro de densidades por vizinhança fixa 
atuará em uma região menor, se considerado sob a ótica de um único elemento finito, conforme mostra a Figura 3.4.

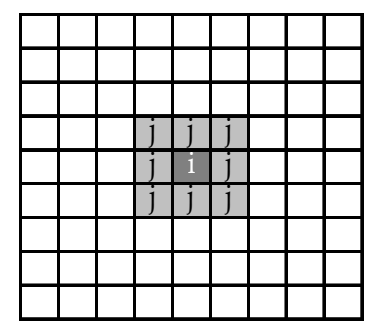

Figura 3.4 - Redução da área de influência do filtro em relação ao elemento finito i.

Este comportamento explica a incapacidade deste tipo de filtro em proporcionar soluções independentes do nível de refinamento da malha.

No filtro com raio de abrangência, a densidade dos elementos - cujos centróides estejam no interior de uma circunferência de centro coincidente com o centróide de um determinado elemento finito central - é capaz de influenciar a densidade do elemento finito central, conforme fica mais claro através da observação da Figura 3.5.

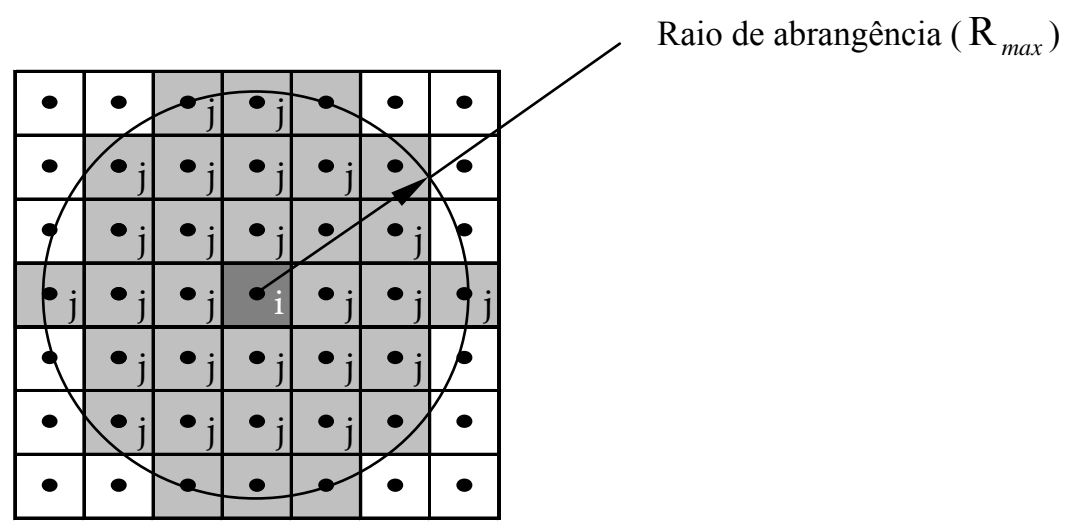

Figura 3.5 - Elemento central i sofrendo a influência dos demais elementos cujos centróides estão no interior da circunferência.

A eq.(3.16) define a maneira pela qual esta influência ocorre.

$$
\rho_{\mathrm{i}}^{\text {ajustado }}=\frac{\rho_{\mathrm{i}} \mathrm{V}_{\mathrm{i}}+\bar{w} \sum_{\mathrm{j}=1}^{\mathrm{nv}} \rho_{\mathrm{j}} \mathrm{V}_{\mathrm{j}}}{\mathrm{V}_{\mathrm{i}}+\bar{w} \sum_{\mathrm{j}=1}^{\mathrm{nv}} \mathrm{V}_{\mathrm{j}}}
$$


O peso $\bar{w}$ é escrito através da seguinte equação:

$$
\bar{w}=\frac{\sum_{\mathrm{j}=1}^{\mathrm{nv}} w_{\mathrm{j}}}{\mathrm{nv}}
$$

onde:

$$
w_{\mathrm{j}}=\frac{\mathrm{R}_{\text {max }}-\mathrm{R}_{\mathrm{ij}}}{\mathrm{R}_{\text {max }}}
$$

na eq.(3.16), nv representa o número de elementos finitos cujos centróides estão no interior da circunferência de raio de abragência $\mathrm{R}_{\max }$. Na eq.(3.18), $\mathrm{R}_{\mathrm{ij}}$ é a distância entre os centróides dos elementos i e j. Se houver um refinamento da malha inicialmente representada pela Figura 3.5, desde que o raio de abragência seja mantido, a região preenchida por elementos que influenciam o elemento central continuará apresentando praticamente a mesma área, nestas condições, haverá uma considerável redução da dependência da solução com relação ao nível de discretização da malha de EF, adicionalmente, é possível evitar também a instabilidade de tabuleiro.

A aplicação do filtro espacial de densidades deve ser feita sobre os limites móveis, ou seja, na preparação do subproblema de otimização. Inicialmente, os limites móveis são calculados em função das tolerâncias percentuais em relação às variáveis de projeto. Posteriormente, tais limites móveis são ajustados através da aplicação das equações (3.18), (3.17) e (3.16), nesta ordem. São estes valores ajustados que devem ser considerados como $\rho_{\mathrm{i}_{\min }}$ e $\rho_{\mathrm{i}_{\max }}$ na última restrição das formulações (3.13) e (3.14). Assim, aplica-se o filtro antes da solução do subproblema de otimização, caso contrário, o ajuste das densidades através do filtro alteraria o "ótimo" do subproblema. 


\subsection{ESTRUTURA DO CÓDIGO COMPUTACIONAL}

Os problemas de otimização topológica, colocados conforme as formulações (2.57) e (2.60), são solucionados através dos métodos numéricos descritos neste capítulo, contudo, as rotinas de cálculo foram viabilizadas por meio de um código computacional, elaborado em linguagem C (ALBUQUERQUE, 1991), cujo fluxo de dados pode ser visto na Figura 3.6.

Foi utilizado o software comercial de elementos finitos ANSYS, versão 10.0, para a modelagem geométrica dos problemas propostos. Ao final desta etapa, por meio deste software, gera-se um arquivo de texto contendo todas as informações com respeito à malha de EF do domínio fixo, bem como a vinculação de alguns de seus nós - caracterizando as condições de contorno do modelo. Por fim, estão contidos neste arquivo os dados sobre as forças aplicadas no domínio - forças de entrada correspondentes a cada um dos carregamentos considerados. Um segundo arquivo de texto, ou arquivo complementar, traz a estimativa inicial para cada variável de projeto. Nele há também a prescrição do comportamento cinemático do mecanismo flexível, que é definido por meio das restrições impostas ao deslocamento de alguns dos nós pertencentes à malha de EF.

O código computacional lê os dois arquivos citados no parágrafo anterior e, em seqüência, efetua a análise do domínio fixo através do MEF. Para o caso de multiflexibilidade, tem-se um número de análises correspondente ao número de carregamentos. $\mathrm{O}$ cálculo do valor da função objetivo é feito com base nos resultados destas análises. Ao final da iteração, testa-se a convergência em relação ao valor da função objetivo calculado na iteração anterior. No caso da variação percentual ser maior do que um certo valor arbitrado, ou seja, não havendo a convergência, o código prossegue efetuando o cálculo das sensibilidades da função objetivo e das funções dos deslocamentos restringidos. Como discutido na seção 2.7, as sensibilidades das funções dos deslocamentos restringidos e da função objetivo são obtidas através do método adjunto. Assim, fazem-se necessárias as análises por MEF do domínio fixo com respeito aos pseudo-carregamentos definidos pelas equações (2.65) e (2.72). Determinados os limites móveis para cada variável de projeto, neles são aplicados o filtro espacial de densidades, conforme mostrado na seção 3.3. Após estas etapas, monta-se o subproblema linear, solucionando-o através da rotina de PL GLPK. 


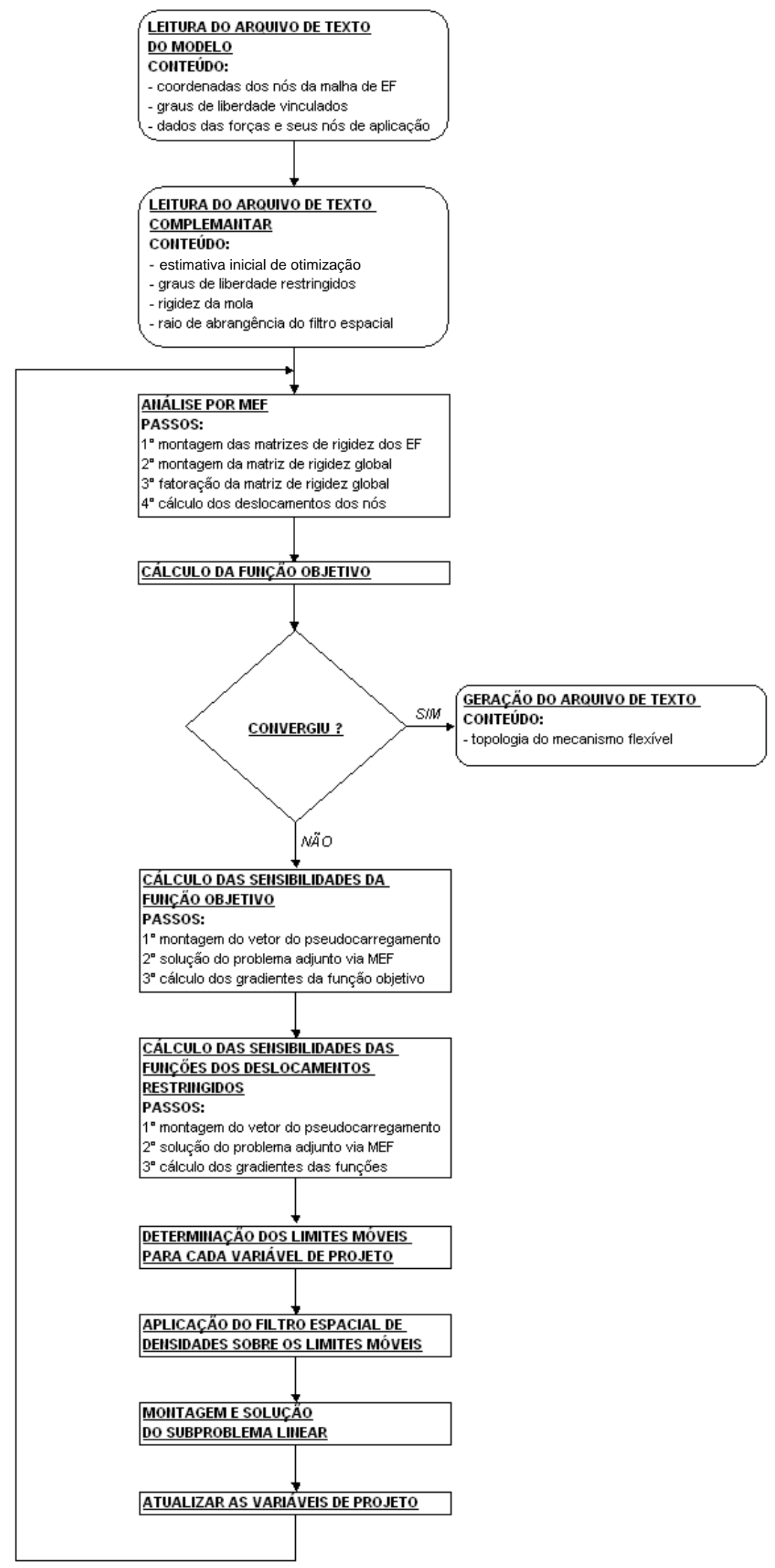

Figura 3.6 - Fluxo de dados do código computacional para o projeto de mecanismos flexíveis. 
Havendo a convergência para uma solução, o código gera um arquivo de texto passível de ser lido pelo software ANSYS, com o qual é possível observar a topologia do mecanismo flexível obtida pelo processo de otimização topológica. 


\section{RESULTADOS E DISCUSSÕES}

\subsection{INTRODUÇÃO}

Este capítulo apresenta os resultados obtidos através da execução do código computacional descrito no capítulo 3. Adotou-se o aço, ou o nylon, como o material base empregado nos processos de otimização. Em cada problema proposto, especificou-se um destes dois materiais. Como se sabe, no caso do aço, os valores usuais do módulo de elasticidade longitudinal $\left(E_{0}\right)$ e da razão de Poisson $\left(v_{0}\right)$ correspondem a $2,1 \times 10^{11} \mathrm{~N} / \mathrm{m}^{2}$ e 0,3, respectivamente. Já para o Nylon, adotou-se: $E_{0}=3 \times 10^{9} \mathrm{~N} / \mathrm{m}^{2}$ e $v_{0}=0,4$. Ainda com respeito ao material base, em problemas nos quais o aço é considerado, convencionou-se que a espessura do domínio fixo deve medir $1 \mathrm{~mm}$. No caso do nylon, este valor passa a ser de $5 \mathrm{~mm}$. Os domínios fixos foram discretizados em EF isoparamétricos quadrados de quatro nós adequados ao estado plano de tensões. O valor adotado para o expoente $n$, presente na eq.(2.55), corresponde a 2 (dois). Contudo, um outro valor $(n=4)$ foi considerado na obtenção de alguns dos resultados demonstrados na seção 4.7. As cotas dimensionais usadas na definição da geometria dos domínios fixos estão escritas em milímetros (mm). Todos os resultados foram extraídos a partir da execução do código de otimização instalado num computador pessoal cujo processador, da marca Intel modelo Centrino, opera na freqüência de 1,73 GHz. Este equipamento apresenta 512 MB de capacidade de memória RAM, o que corresponde a 15 minutos de processamento computacional para que o problema de projeto com maior discretização de malha (4050 elementos finitos) possa ser solucionado.

Na seção 4.2, demonstram-se os resultados preliminares. Neles, certas instabilidades características ao MOT são observadas. Estas instabilidades correspondem à sensibilidade do problema de otimização em relação aos valores iniciais atribuídos às variáveis de projeto sensibilidade em relação à estimativa inicial de otimização -, à dependência de malha e à formação da instabilidade de tabuleiro. Na seção 4.3, os resultados foram obtidos com a ativação do filtro espacial de densidades, observou-se, nestes resultados, que as instabilidades discutidas na seção 4.2 foram minimizadas. A seção 4.4 traz a constatação quanto à redução da ocorrência das “dobradiças” nas topologias obtidas pela formulação (2.57). Na seção 4.5, verifica-se que: ao variar a quantidade de material disponível, ou o raio de abrangência 
empregado no esquema de filtragem, modifica-se a topologia obtida no processo de otimização. Já na seção 4.6, as modificações decorrem da alteração na rigidez da mola ou da alteração na restrição de deslocamento necessário à sua deformação. A seção 4.7 sugere que a formulação discutida neste trabalho também é aplicável ao projeto de estruturas rígidas, para as quais a flexibilidade média máxima pode ser indiretamente prescrita. Esta prescrição se dá através das restrições impostas aos deslocamentos dos pontos onde as forças ativas atuam. Finalmente, na seção 4.8, são demonstradas as configurações de projeto para os mecanismos flexíveis sujeitos à aplicação de mais de um caso de carregamento - adaptação da formulação (2.57) à multi-flexibilidade.

\subsection{RESULTADOS PRELIMINARES}

\subsubsection{Sensibilidade em relação aos valores iniciais atribuídos às variáveis de projeto}

Nesta seção, verifica-se a sensibilidade da formulação (2.57) com respeito aos valores iniciais adotados para as variáveis de projeto. Tendo em vista este objetivo, utilizou-se o problema de otimização topológica representado pela Figura 4.1.

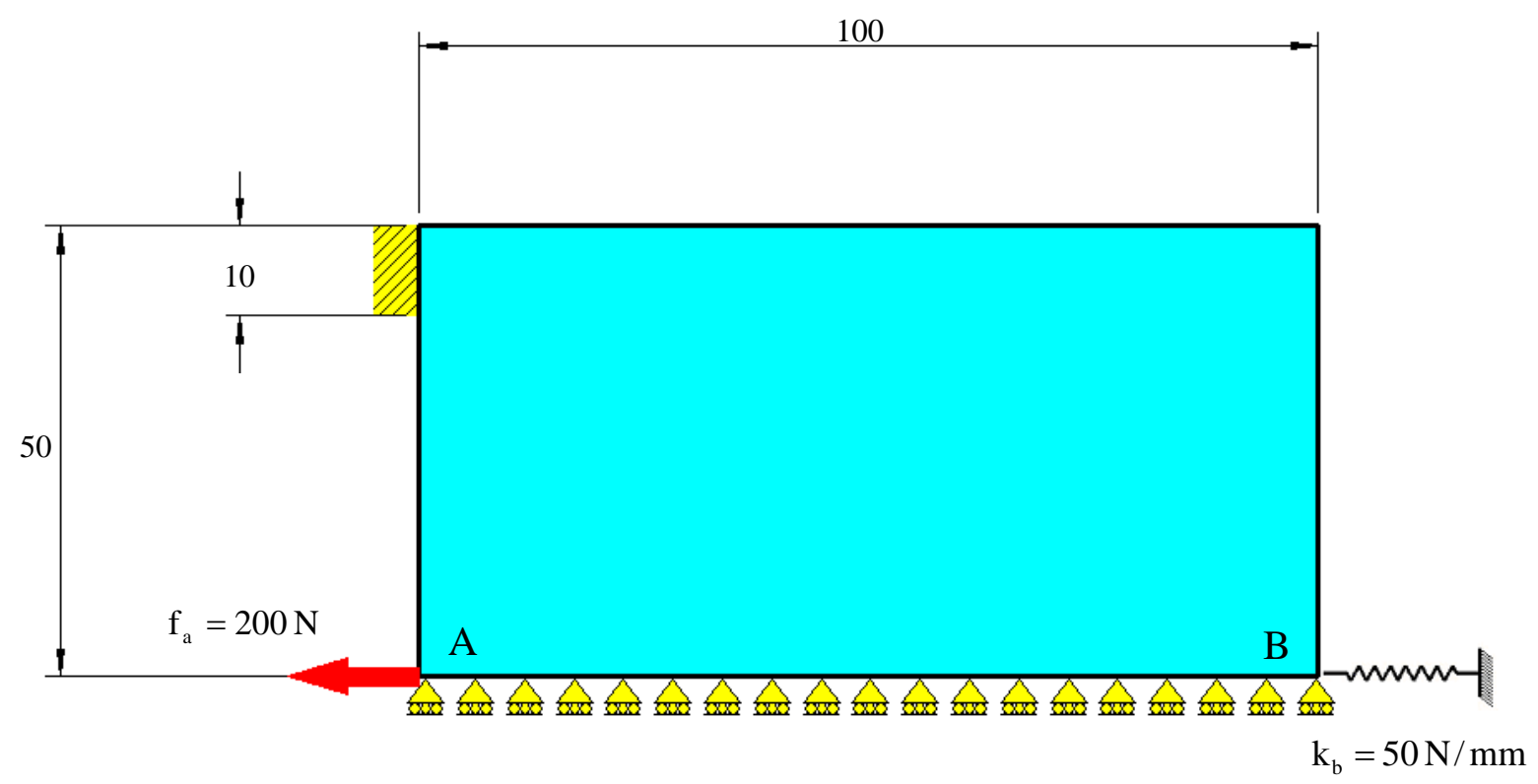

Figura 4.1 - Representação do problema de otimização. 
Considera-se que o material base a ser distribuído no domínio fixo mostrado pela Figura 4.1 será o nylon. A força representada por $\mathrm{f}_{\mathrm{a}}$ deverá provocar um deslocamento máximo de $2 \mathrm{~mm}$ no seu ponto de aplicação, além de um deslocamento mínimo de $1 \mathrm{~mm}$ na compressão da mola de rigidez $\mathrm{k}_{\mathrm{b}}$. Os valores destes parâmetros estão indicados na Figura 4.1. Para solucionar o problema, restringiu-se o material do mecanismo em $15 \%$ do volume do domínio fixo, o qual foi discretizado em 2592 elementos finitos isoparamétricos quadrados de quatro nós. Diferentes estimativas iniciais de otimização foram adotadas entre os três resultados obtidos. Contudo, cada um dos três processos iterativos iniciou com um único valor atribuído a todas as variáveis de projeto, sendo 0,001 o valor correspondente ao primeiro caso, 0,500 para o segundo e 1,000 para o terceiro. Em todos os problemas propostos nesta seção 4.2, não se fez uso do filtro espacial de densidades, já que o objetivo era verificar a formulação em sua essência, ou seja, sem os artifícios numéricos utilizados para a obtenção de resultados mais estáveis. Assim, as Figuras 4.2, 4.3 e 4.4 ilustram as topologias obtidas em relação a este primeiro problema proposto.

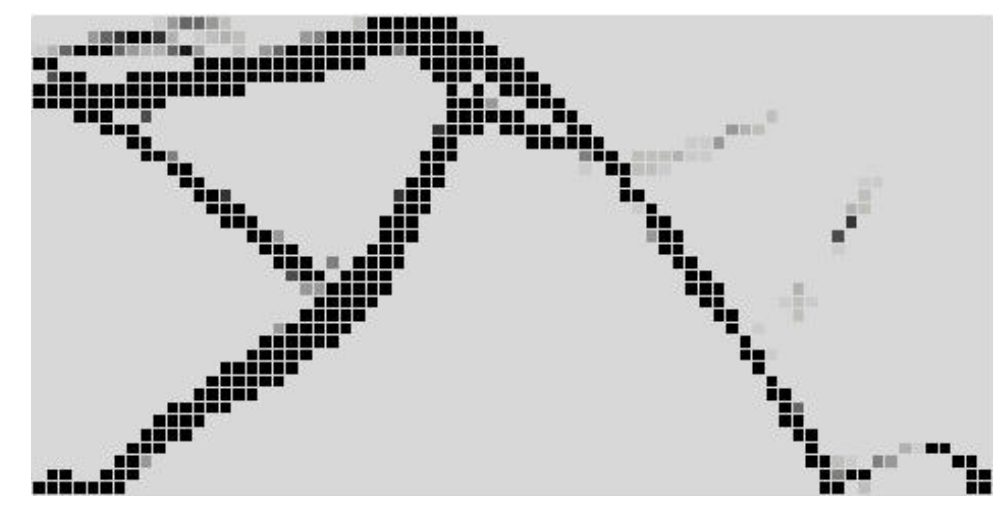

Figura 4.2 - Topologia obtida a partir de $\rho_{\text {einicial }}=0,001$.

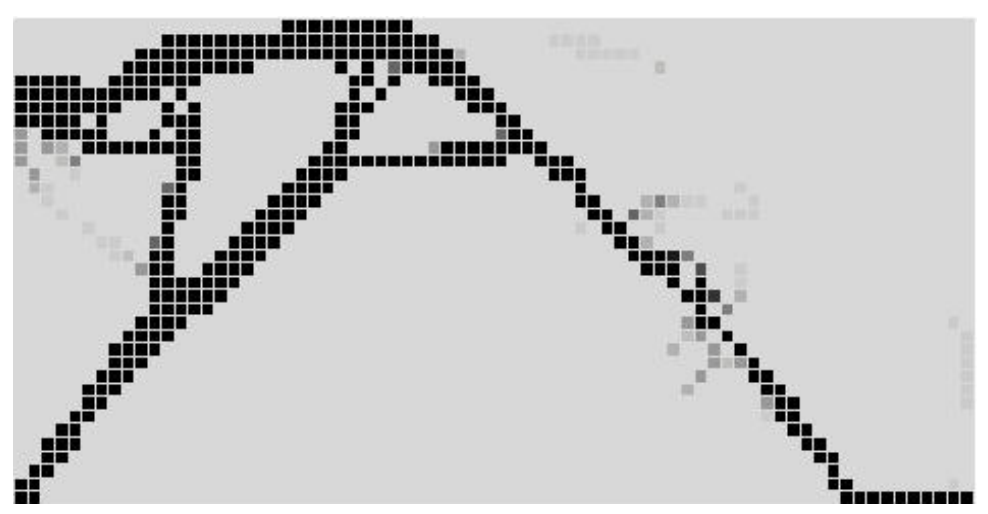

Figura 4.3 - Topologia obtida a partir de $\rho_{\text {einicial }}=0,500$. 


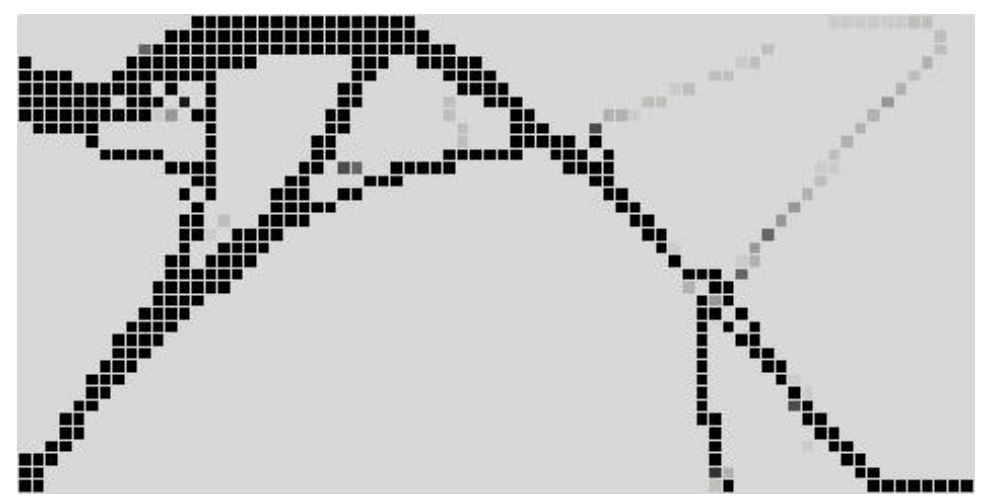

Figura 4.4 - Topologia obtida a partir de $\rho_{\text {einicial }}=1,000$.

Encontrou-se, ainda, uma quarta topologia, vide Figura 4.5. Neste caso, a estimativa inicial de otimização atribuiu, a cada uma das 2592 variáveis de projeto, um valor específico definido aleatoriamente.

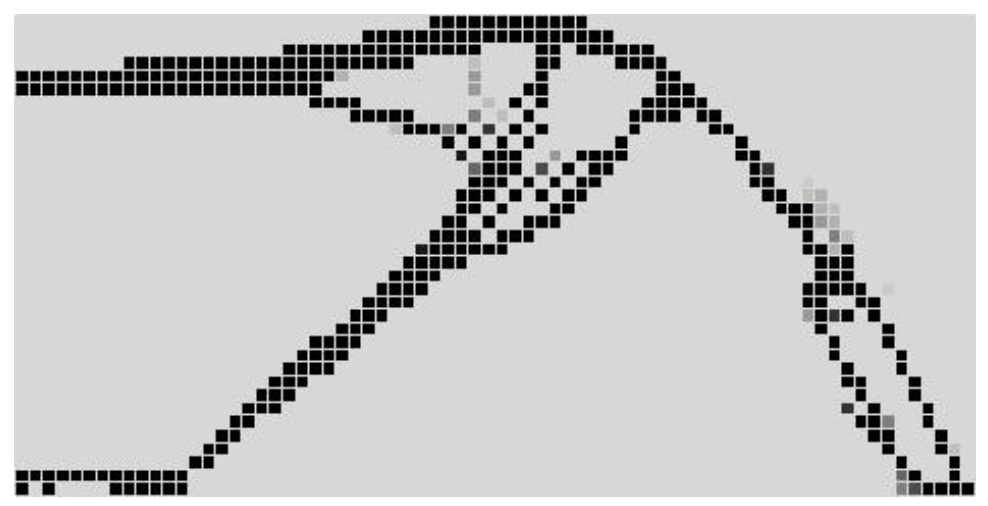

Figura 4.5 - Topologia obtida a partir de valores aleatórios atribuídos às variáveis de projeto.

Estes resultados demonstram que a formulação (2.57) produziu diferentes topologias, o que indica a existência de várias soluções para um mesmo problema de otimização - são as chamadas soluções locais ou "ótimos” locais do problema não-convexo (ROCKAFELLAR, 1997). A solução local que se apresentará ao final do processo iterativo dependerá dos valores adotados para as variáveis de projeto no início da otimização. 


\subsubsection{Sensibilidade em relação ao refinamento da malha do domínio fixo}

A Figura 4.6 representa o problema de otimização topológica cujo objetivo é mostrar o comportamento da formulação (2.57) em relação ao refinamento da malha de elementos finitos.

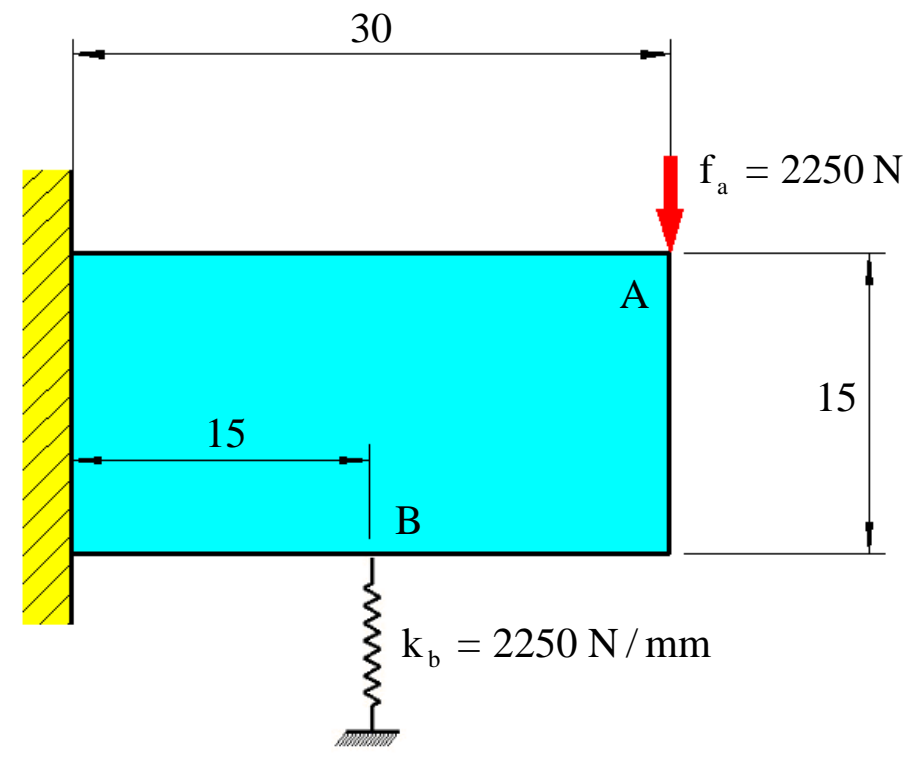

Figura 4.6 - Representação do problema de otimização.

Considera-se que o material base a ser distribuído no domínio fixo de projeto será o aço. A força representada por $\mathrm{f}_{\mathrm{a}}$ deverá provocar um deslocamento máximo de $2 \mathrm{~mm}$ no seu ponto de aplicação, além de um deslocamento mínimo de $1 \mathrm{~mm}$ na compressão da mola de rigidez $\mathrm{k}_{\mathrm{b}}$. Os valores destes parâmetros estão indicados na Figura 4.6. A fim de solucionar o problema, restringiu-se o material do mecanismo em $20 \%$ do volume do domínio fixo, para o qual três discretizações distintas foram aplicadas - 1250, 2450 e 2888 elementos finitos. Em cada um dos três casos, o processo de otimização foi iniciado a partir da mesma estimativa: 0,15 para todas as variáveis de projeto. As Figuras 4.7, 4.8 e 4.9 correspondem às topologias obtidas. 


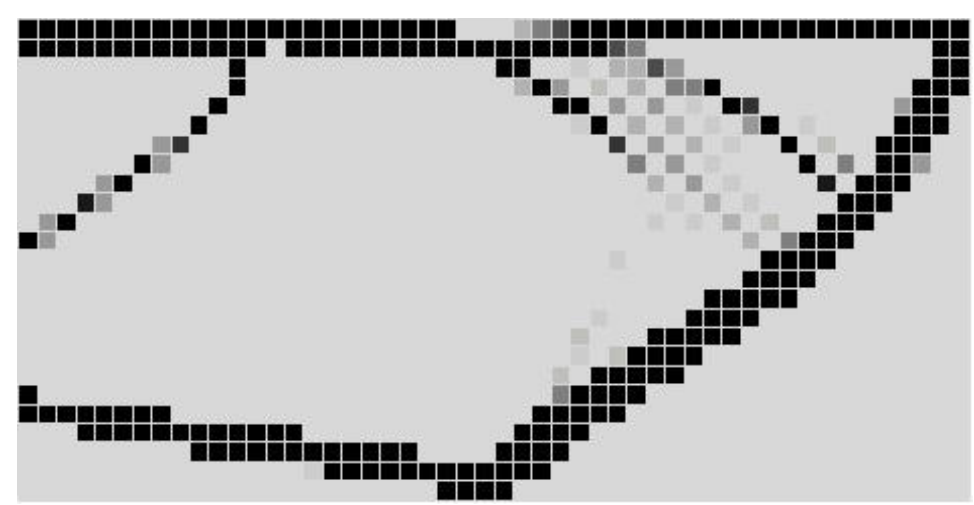

Figura 4.7 - Topologia obtida com a discretização de 1250 elementos.

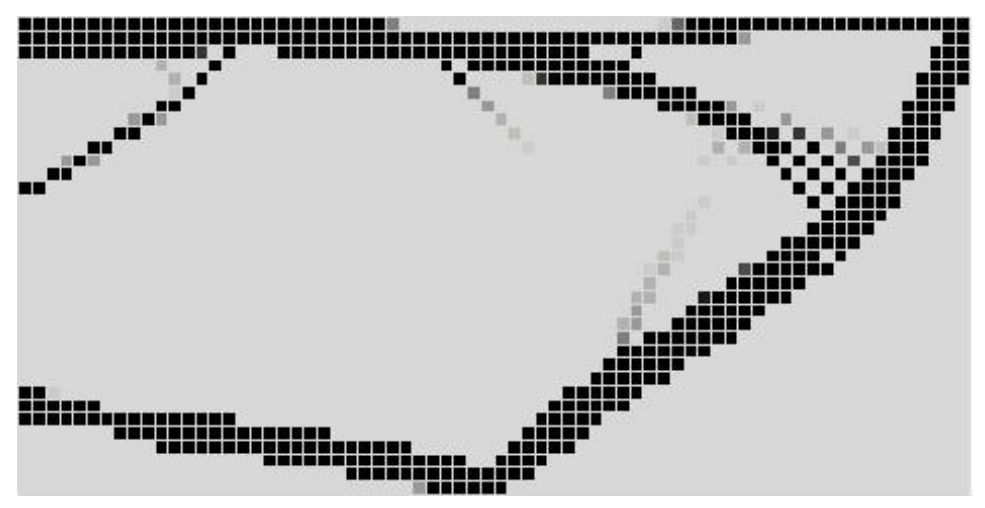

Figura 4.8 - Topologia obtida com a discretização de 2450 elementos.

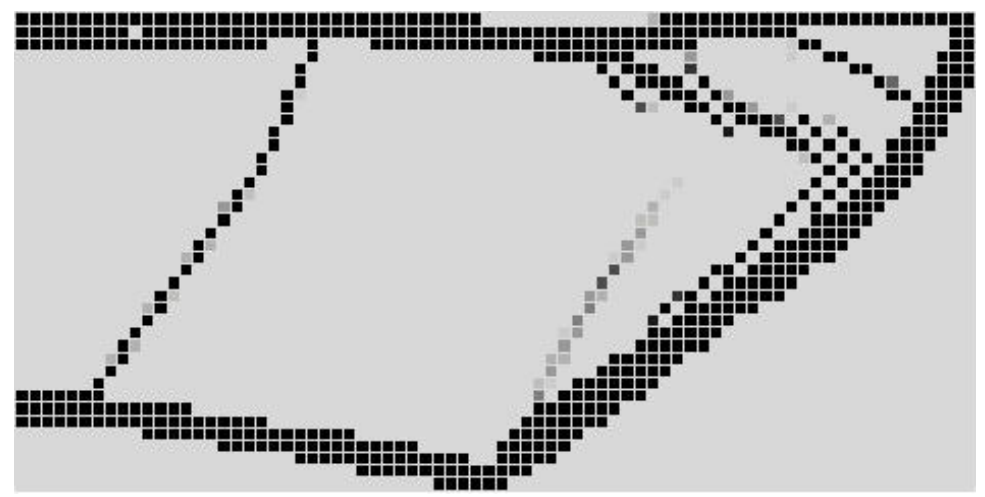

Figura 4.9 - Topologia obtida com a discretização de 2888 elementos.

Os resultados demonstram as diferenças entre as três topologias, indicando que a formulação é sensível à discretização do domínio fixo de projeto. Este comportamento pode ser explicado pela penalização imposta à energia de deformação dos elementos finitos da malha, conforme mostra a eq.(2.51). Assim, ainda que a parametrização linear do tensor constitutivo tenha sido adotada, o problema de projeto de mecanismos flexíveis manterá a sua natureza discreta e, portanto, não convergirá a uma solução (RIETZ, 2001). 
Observa-se que em determinadas regiões das topologias mostradas pelas Figuras 4.7, 4.8 e 4.9 há uma tendência à formação da instabilidade de tabuleiro. Na próxima seção, temse um exemplo em que a instabilidade de tabuleiro aparece de forma mais efetiva.

\subsubsection{Ocorrência da instabilidade de tabuleiro}

Para verificar a ocorrência da instabilidade de tabuleiro nas topologias geradas pela formulação (2.57), utilizou-se o problema de otimização topológica mostrado na Figura 4.10.

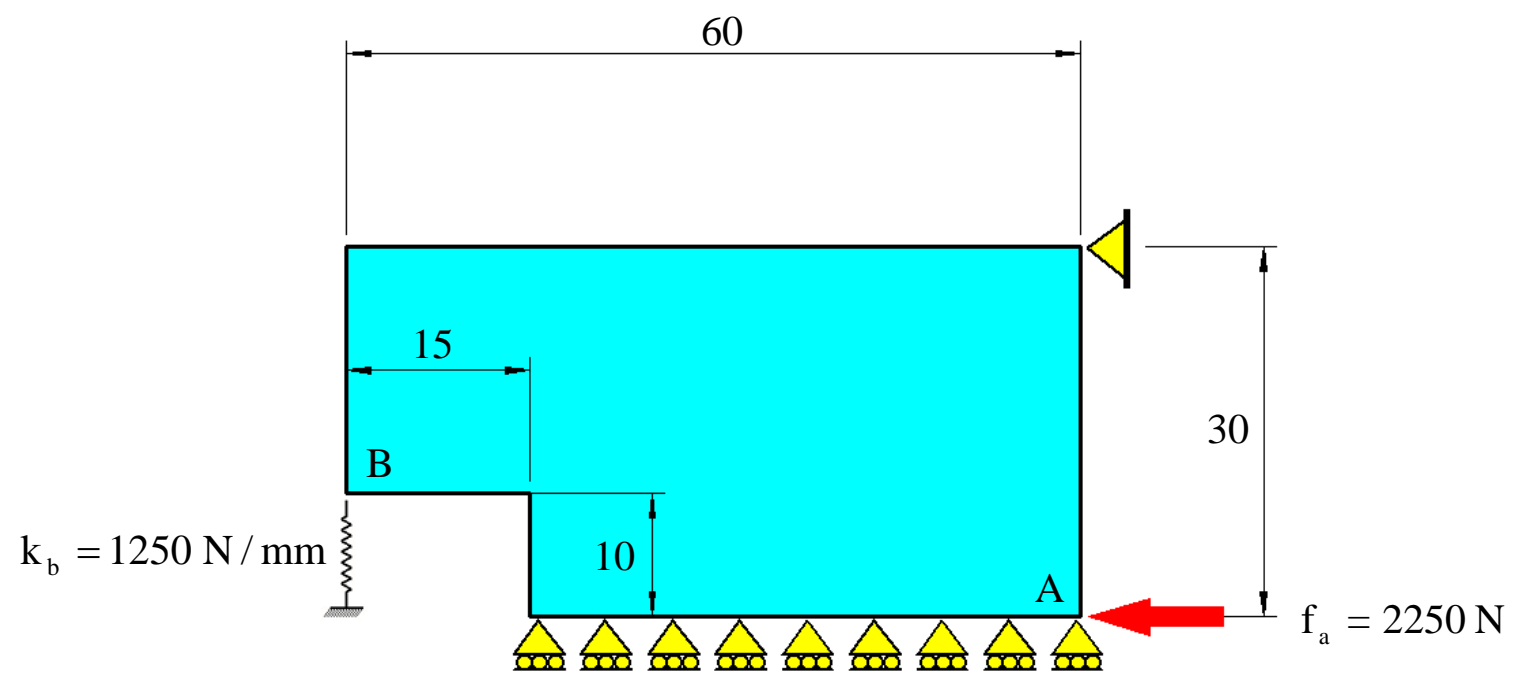

Figura 4.10 - Representação do problema de otimização.

Considera-se que o material base a ser distribuído no domínio fixo de projeto será o aço. A força representada por $\mathrm{f}_{\mathrm{a}}$ deverá provocar um deslocamento máximo de $2 \mathrm{~mm}$ no seu ponto de aplicação, além de um deslocamento mínimo de $1 \mathrm{~mm}$ na compressão da mola de rigidez $\mathrm{k}_{\mathrm{b}}$. Os valores destes parâmetros estão indicados na Figura 4.10. Restringiu-se o material do mecanismo em 35\% do volume do domínio fixo, que foi discretizado em 1650 elementos finitos. A topologia mostrada na Figura 4.11 foi obtida a partir de uma estimativa inicial cujo valor, correspondente a 0,15 , foi atribuído a todas as variáveis de projeto. 


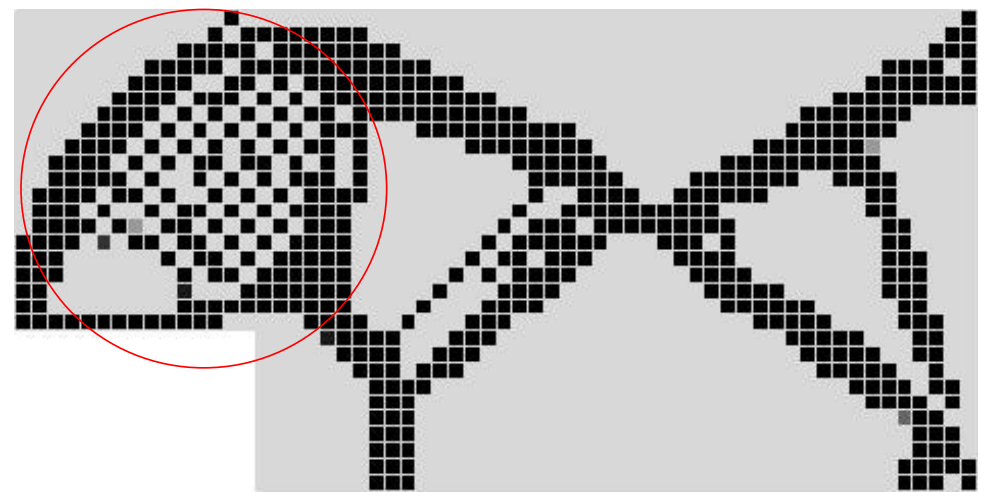

Figura 4.11 - Presença da instabilidade de tabuleiro.

A área destacada na Figura 4.11 apresenta uma região na qual a formação da instabilidade de tabuleiro ocorre. Trata-se de um resultado importante para a compreensão do assunto quando estudado à luz da formulação demonstrada na seção 2.6.4. Diaz e Sigmund (1995) defendem a idéia de que os elementos finitos de baixa ordem, tais como os isoparamétricos quadrados de quatro nós usados neste trabalho - cujas funções de interpolação são bi-lineares -, apresentam maior rigidez ao cisalhamento se dispostos na forma de “tabuleiro de xadrez”. A idéia pode explicar o resultado observado acima, pois, intuitivamente, conclui-se que é necessário haver maior rigidez na região destacada pela Figura 4.11, já que desta forma o comportamento cinemático do mecanismo poderá atender às restrições de deslocamento impostas pelo problema de otimização. Adiante, na seção 4.3.4, a Figura 4.25 mostra a deformação de um mecanismo cuja geometria foi definida a partir da interpretação de outra solução encontrada para este problema. Esta nova solução corresponde à topologia da Figura 4.22, na qual a instabilidade de tabuleiro foi substituída por reforços estruturais presentes devido à aplicação do filtro espacial de densidades durante o processo de otimização. Os reforços mantêm a rigidez necessária para que esta região do mecanismo resista às tensões geradas a partir da deformação da mola. Na próxima seção deste capítulo, serão discutidos os diferentes aspectos decorrentes da utilização do filtro espacial de densidades. Em relação à formulação (2.57), um possível recurso para evitar a ocorrência da instabilidade de tabuleiro é o aumento de material disponível ao processo de otimização, o que corresponderia a uma outra maneira de enrijecer a região do mecanismo que está acoplada à mola. 


\subsection{RESULTADOS OBTIDOS COM A ATIVAÇÃO DO FILTRO}

\subsubsection{Comentários iniciais}

A partir deste ponto do capítulo 4, todas as topologias apresentadas foram obtidas com a ativação do filtro espacial de densidades. Porém, particularmente nesta seção 4.3, os aspectos decorrentes da utilização deste expediente serão discutidos. Todos os problemas de otimização topológica considerados na seção 4.2 são novamente adotados a fim de que os resultados possam ser comparados. Na seção 4.3.2, verifica-se o efeito do filtro na sensibilidade da solução em relação à estimativa inicial de otimização. A independência de malha e a redução da ocorrência da instabilidade de tabuleiro são observadas nas seções 4.3.3 e 4.3.4, respectivamente.

\subsubsection{Problema não-convexo}

Com o propósito de averiguar a influência do filtro espacial de densidades sobre a sensibilidade da solução em relação aos valores iniciais atribuídos às variáveis de projeto, o problema definido na seção 4.2.1 foi novamente solucionado para cada uma das quatro estimativas iniciais de otimização empregadas naquela seção. O raio de abrangência adotado no esquema de filtragem foi de 1,75 mm nos quatro casos. Assim, as Figuras 4.12, 4.13, 4.14 e 4.15 ilustram as topologias obtidas.

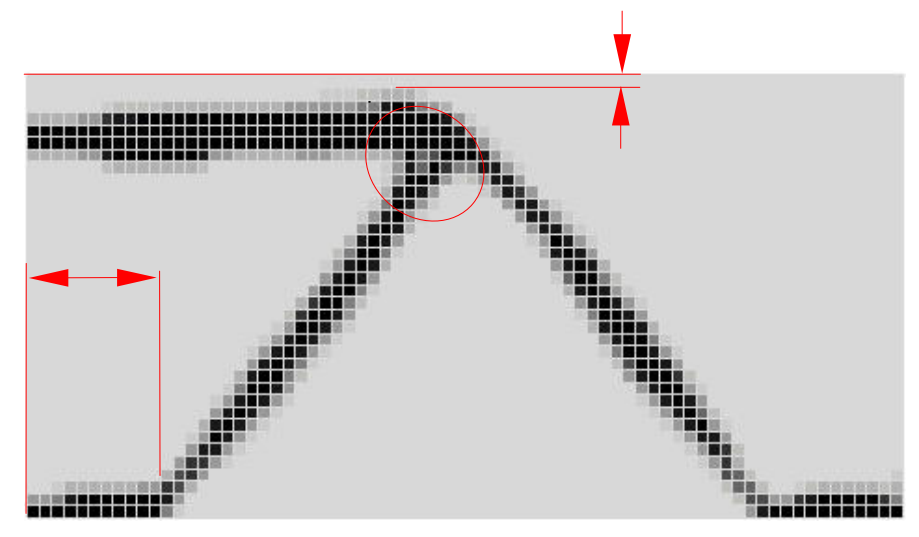

Figura 4.12 - Topologia obtida a partir de $\rho_{\text {einicial }}=0,001$ e uso do filtro espacial de densidades. 


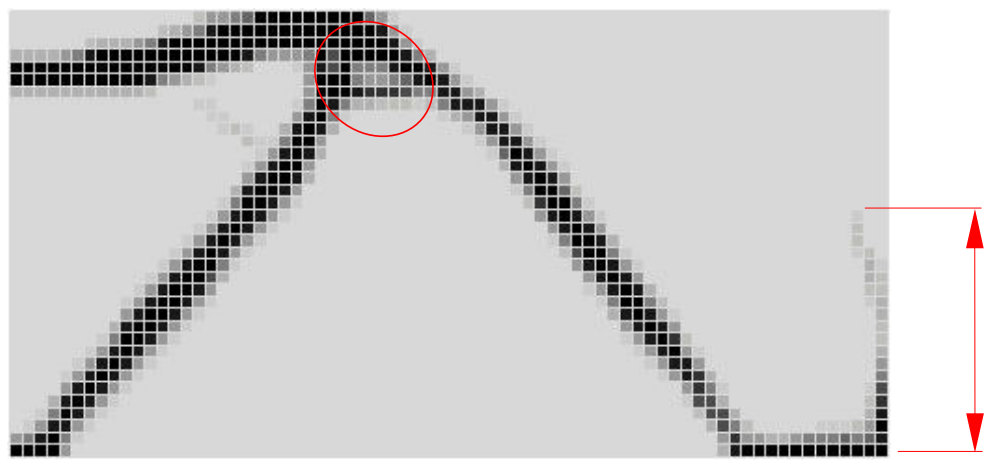

Figura 4.13 - Topologia obtida a partir de $\rho_{\text {einicial }}=0,500$ e uso do filtro espacial de densidades.

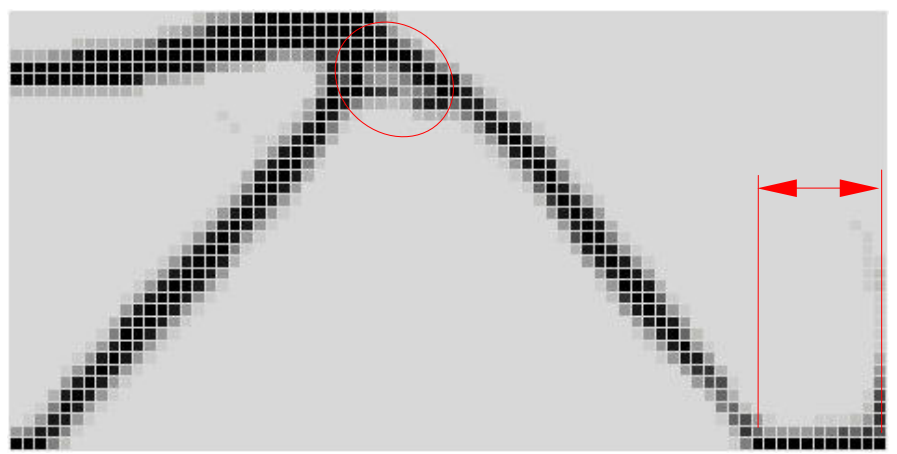

Figura 4.14 - Topologia obtida a partir de $\rho_{\text {einicial }}=1,000$ e uso do filtro espacial de densidades.

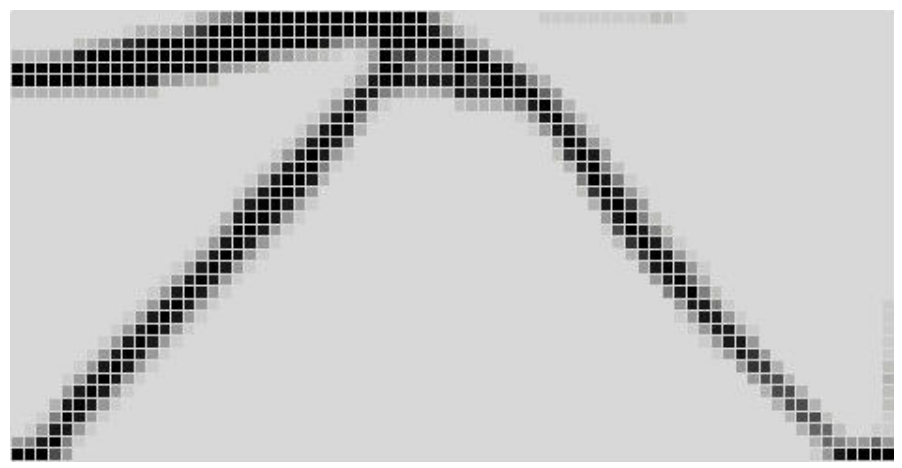

Figura 4.15 - Topologia obtida a partir de valores aleatórios atribuídos às variáveis de projeto e uso do filtro espacial de densidades.

Os detalhes destacados pelas elipses, bem como as cotas referenciais, indicam as diferenças entre as quatro topologias. Tais resultados demonstram que o filtro espacial de densidades não é um recurso pelo qual a não-convexidade do problema de otimização possa ser contornada, ou seja, mesmo com o filtro ativado, o problema mantém a sua característica de apresentar vários “ótimos” locais.

Do ponto de vista da Engenharia, a não-convexidade do problema de otimização não chega a ser um inconveniente, pois qualquer um dos “ótimos” encontrados, desde que sejam 
factíveis sob os aspectos construtivo e funcional, são aceitos para o uso prático. Assim, a preocupação recai sobre os problemas numéricos que inviabilizam as topologias, os mais comuns, no caso dos mecanismos flexíveis, são a instabilidade de tabuleiro e a presença das “dobradiças”.

\subsubsection{Independência de malha}

A fim de avaliar a eficiência do filtro espacial de densidades aplicado à redução da dependência de malha, o problema de projeto de mecanismo flexível proposto na seção 4.2.2 foi novamente solucionado para cada uma das três discretizações impostas ao domínio fixo. O raio de abrangência adotado no esquema de filtragem foi de $0,75 \mathrm{~mm}$ nos três casos. A seguir, têm-se as topologias obtidas, cada uma das quais seguida por um conjunto de quatro gráficos de convergência que registram o histórico do processo de otimização. Os gráficos correspondem à função objetivo, ao volume de material, ao deslocamento do ponto de aplicação da força (entrada) e ao deslocamento do ponto vinculado à mola (saída), todos representados em função do número de iterações. Assim, em relação ao primeiro caso de discretização, têm-se as Figuras 4.16 e 4.17.

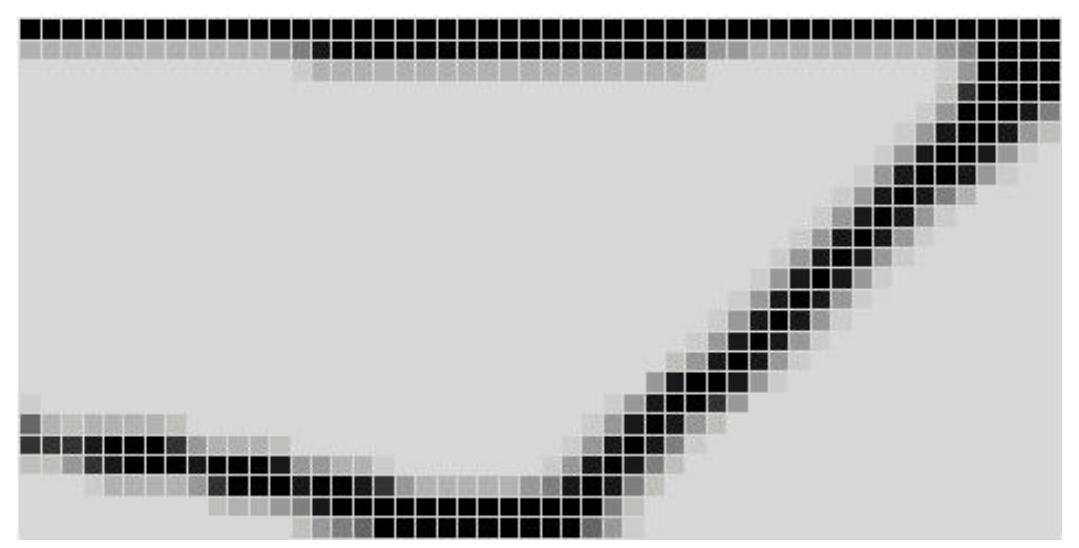

Figura 4.16 - Topologia obtida com a discretização de 1250 elementos e uso do filtro espacial de densidades. 

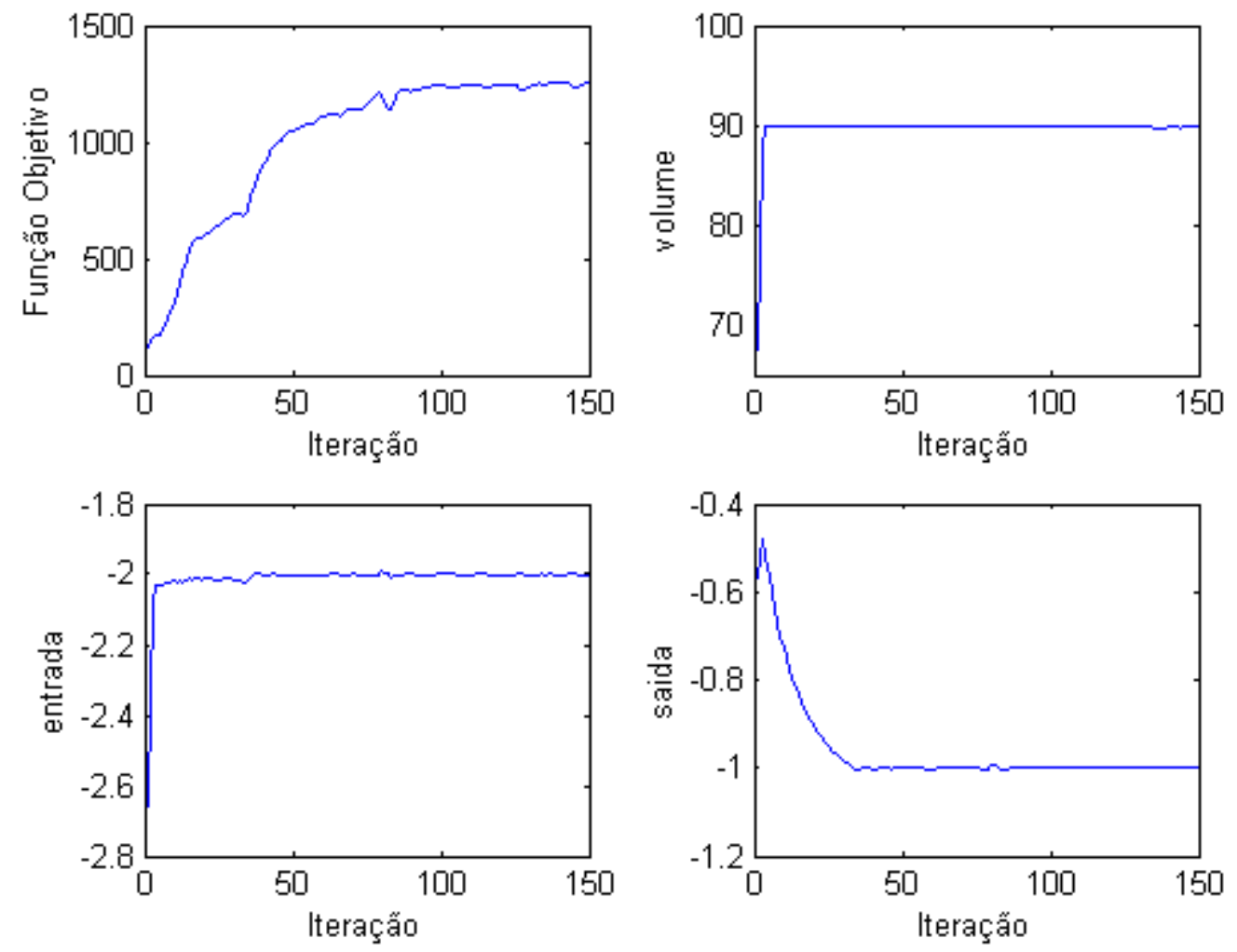

Figura 4.17 - Gráficos de convergência referentes à topologia da Figura 4.16.

Em relação ao segundo caso de discretização, observam-se as Figuras 4.18 e 4.19.

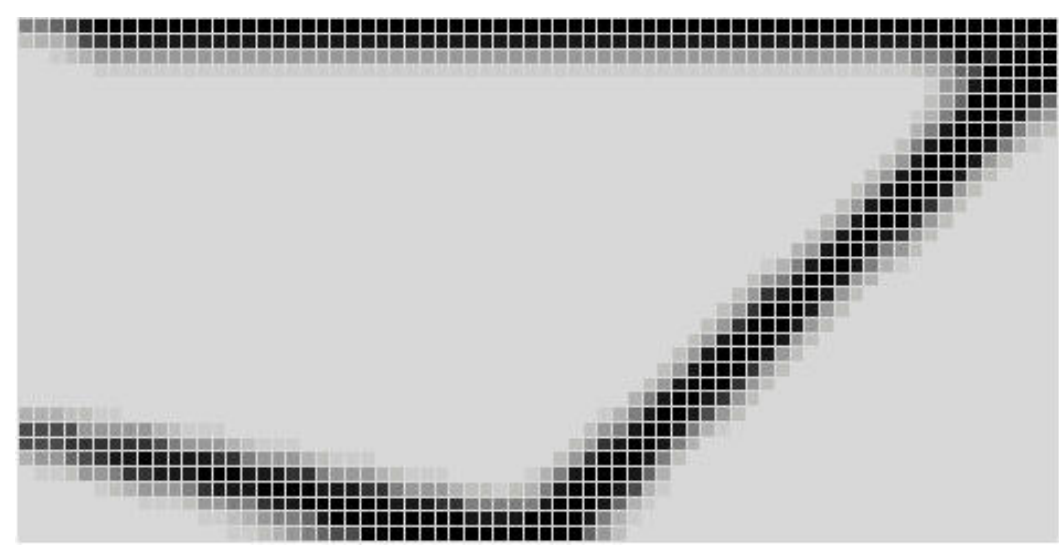

Figura 4.18 - Topologia obtida com a discretização de 2450 elementos e uso do filtro espacial de densidades. 

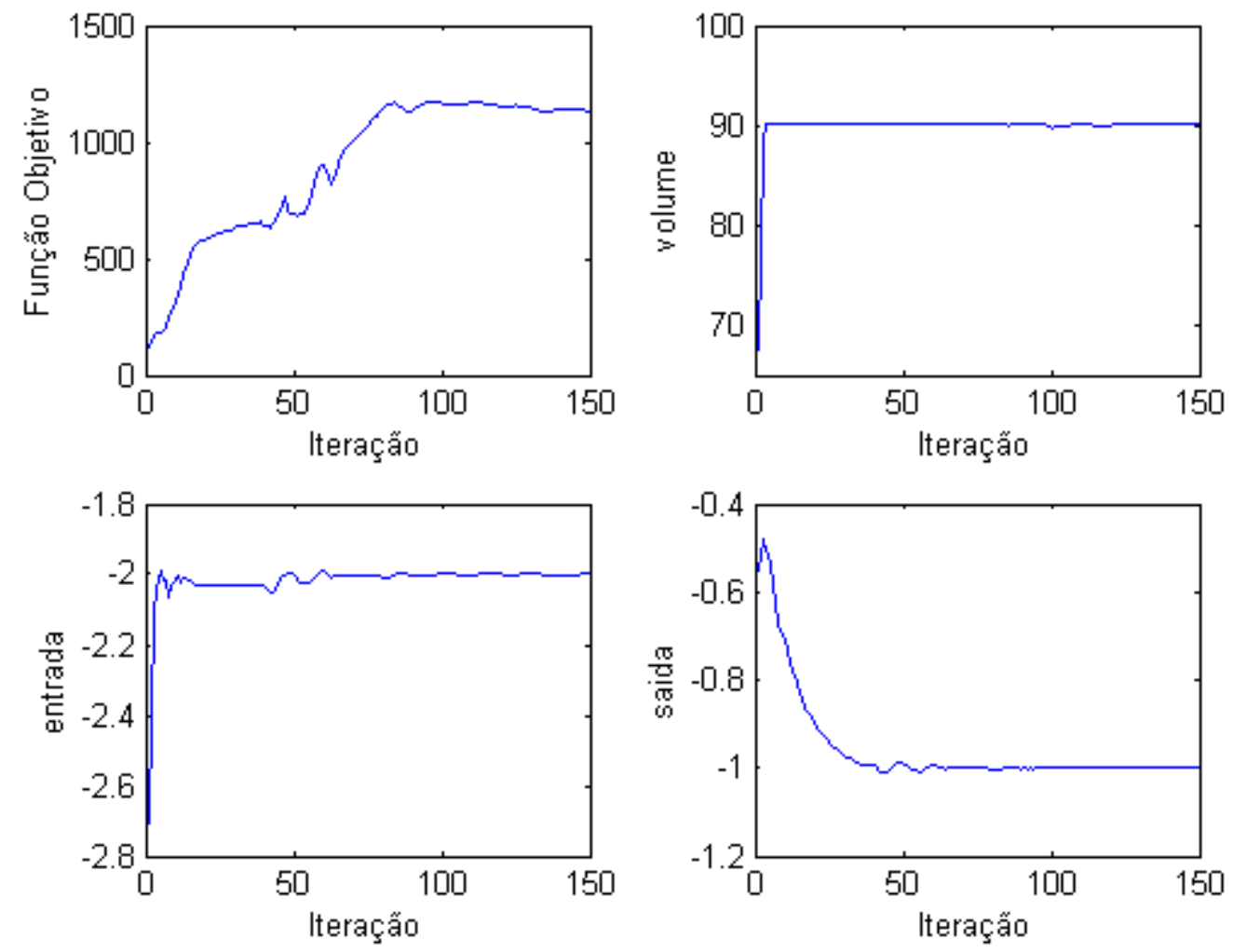

Figura 4.19 - Gráficos de convergência referentes à topologia da Figura 4.18.

Em relação ao segundo caso de discretização, têm-se, por fim, as Figuras 4.20 e 4.21.

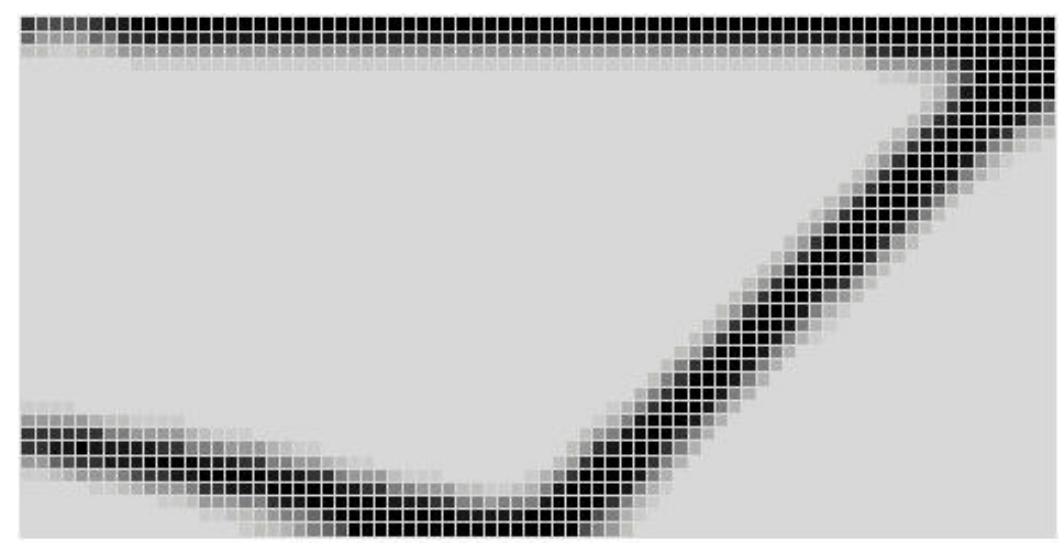

Figura 4.20 - Topologia obtida com a discretização de 2888 elementos e uso do filtro espacial de densidades. 

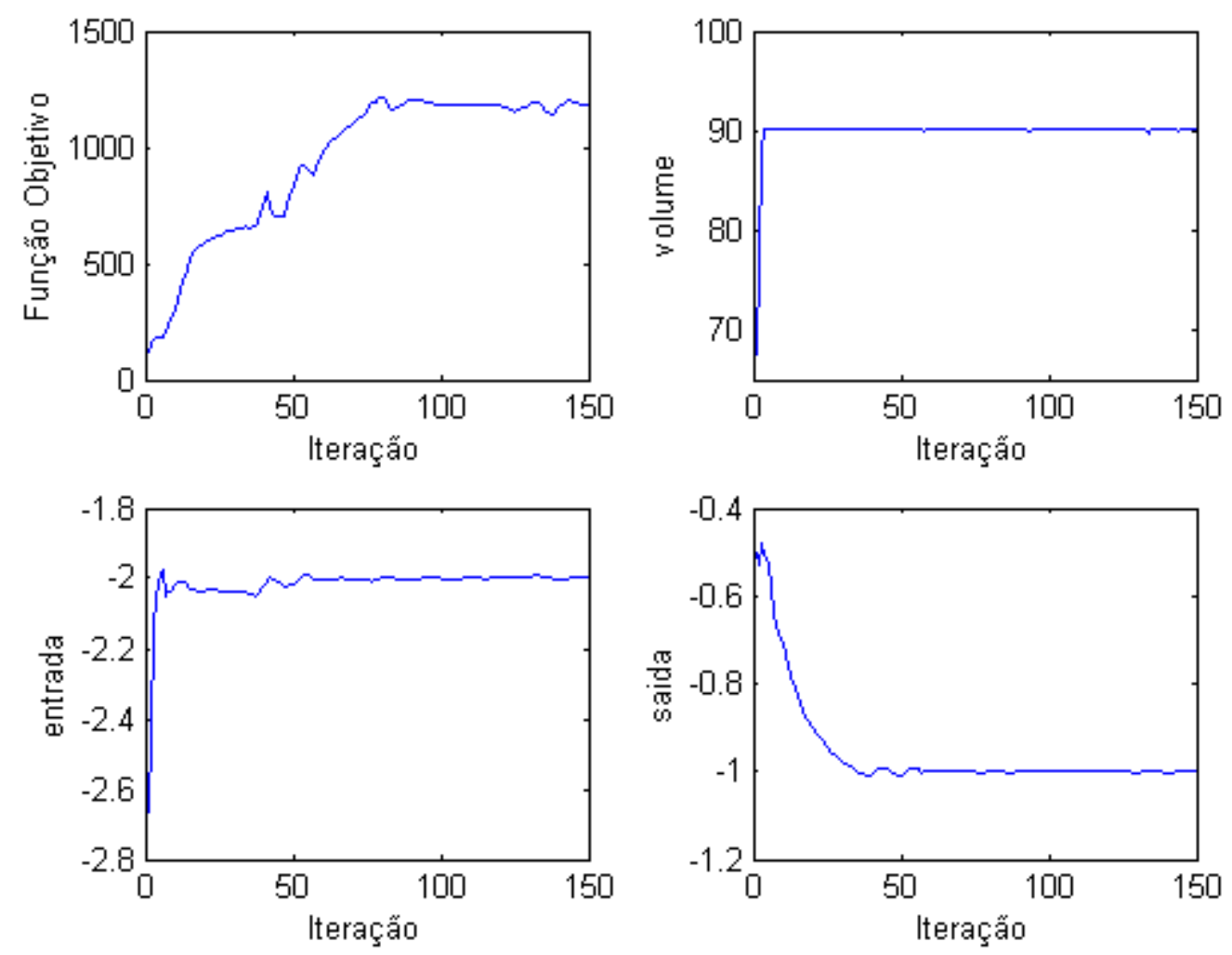

Figura 4.21 - Gráficos de convergência referentes à topologia da Figura 4.20.

Observa-se que, não obstante a diferença no refinamento das malhas, as topologias das Figuras 4.16, 4.18 e 4.20 são muito semelhantes. O filtro espacial de densidades simplificou os seus aspectos se comparados às topologias demonstradas pelas Figuras 4.7, 4.8 e 4.9, respectivamente. Quanto aos gráficos de convergência, nota-se que têm praticamente as mesmas características nas três situações, vide Figuras 4.17, 4.19 e 4.21. Em cada tipo particular de parâmetro analisado - seja a função objetivo, ou cada uma das restrições do problema -, os gráficos convergem aproximadamente para um mesmo valor, o que demonstra consistência em relação ao fato de que as três topologias representam um único “ótimo” local, apesar de terem sido obtidas a partir de discretizações distintas.

\subsubsection{Redução da instabilidade de tabuleiro}

Como já foi discutido na seção 2.3.6, o filtro espacial de densidades, além de manter a independência de malha, é útil no propósito de reduzir a ocorrência da instabilidade de 
tabuleiro. Para ilustrar o fato, o problema de projeto de mecanismo flexível proposto na seção 4.2.3 foi novamente considerado. Contudo, em sua solução, ativou-se o filtro espacial de densidades, para o qual o raio de abrangência adotado foi de 1,05 mm. A Figura 4.22 mostra a nova topologia obtida.

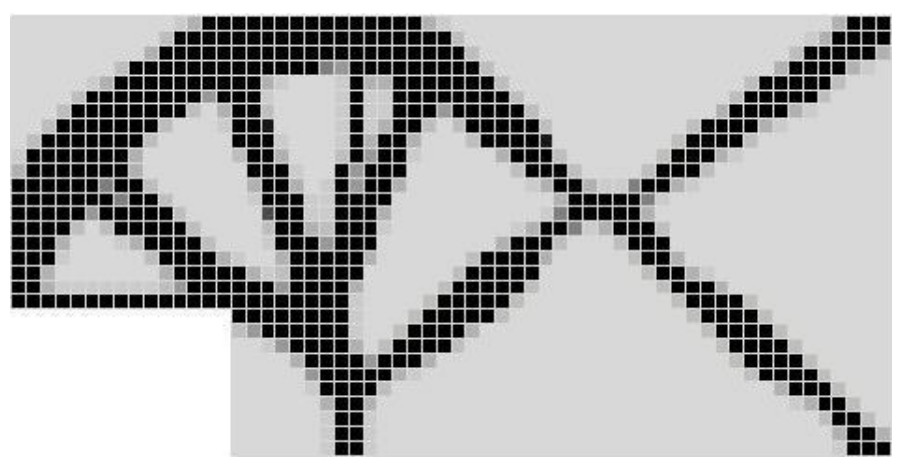

Figura 4.22 - Topologia obtida com $\mathrm{R}_{\max }=1,05 \mathrm{~mm}$.

O filtro espacial de densidades removeu a instabilidade de tabuleiro inicialmente presente na topologia da Figura 4.11. A simplificação imposta pelo filtro substituiu a instabilidade por reforços. Já os gráficos de convergência indicam que os deslocamentos de entrada e saída assumiram, respectivamente, os valores mínimo e máximo prescritos pelo problema (restrições ativas ao final do processo de otimização), vide Figura 4.23.
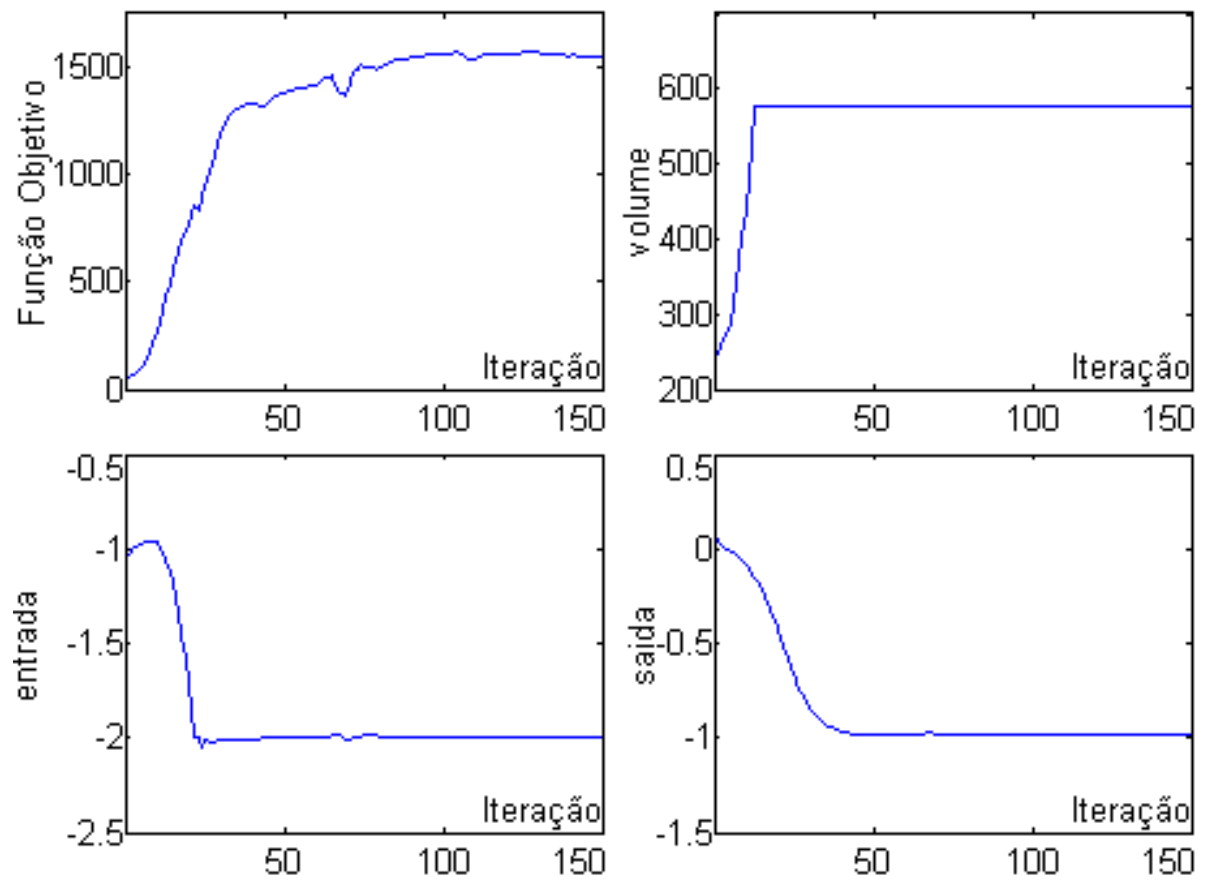

Figura 4.23 - Gráficos de convergência referentes à topologia obtida com $\mathrm{R}_{\max }=1,05 \mathrm{~mm}$. 
Para confirmar o comportamento cinemático determinado pelo problema proposto, a topologia tida como solução foi interpretada, dando origem a um modelo de elementos finitos sobre o qual se efetuou uma análise estática ${ }^{1}$. O modelo correspondente à interpretação feita sobre a topologia da Figura 4.22 é representado pela Figura 4.24.

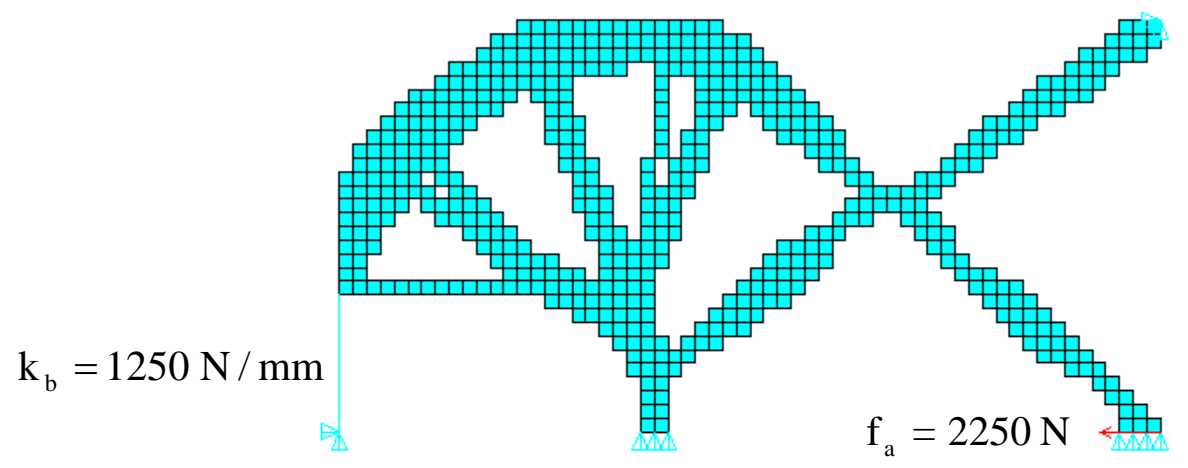

Figura 4.24 - Interpretação da solução apresentada na Figura 4.22.

Fez-se a interpretação de forma sistêmica, pois os elementos finitos com densidade inferior a 0,5, pertencentes à topologia da Figura 4.22, foram desconsiderados na elaboração do modelo da Figura 4.24. Contudo, aos elementos que apresentaram densidade superior ou igual a 0,5, atribuíram-se as propriedades elásticas do material base. Para completar o modelo, representou-se a mola por uma barra cujos parâmetros (material constitutivo, área da secção transversal e o comprimento) foram ajustados de modo que a rigidez $\mathrm{k}_{\mathrm{b}}$ pudesse ser estabelecida. Neste trabalho, todas as interpretações feitas sobre as topologias seguiram o critério descrito acima.

B

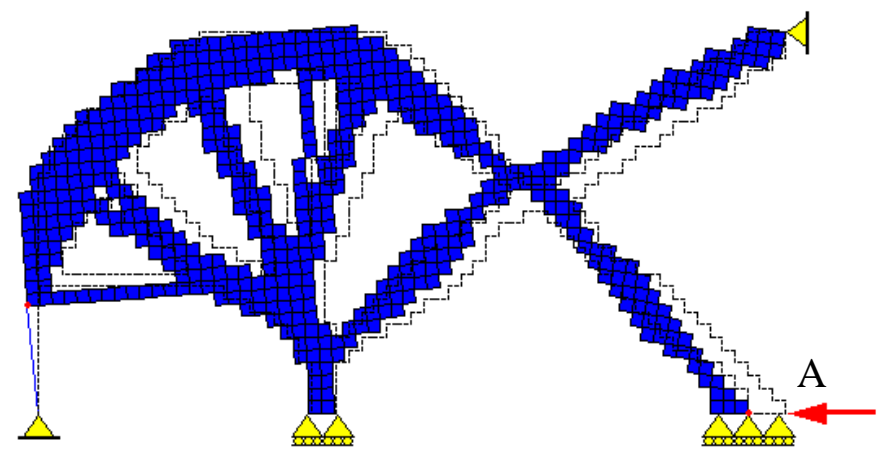

Figura 4.25 - Configuração deformada.

\footnotetext{
${ }^{1} \mathrm{O}$ carregamento definido pelo problema de otimização é considerado na análise estática efetuada sobre o mecanismo flexível do modelo.
} 
A Figura 4.25 mostra o resultado da análise estática efetuada sobre o modelo demonstrado na Figura 4.24. Através deste resultado é possível verificar se a geometria do mecanismo atende às necessidades de projeto, as quais foram prescritas na definição do problema de otimização. Outros resultados deste tipo, decorrentes da análise de outros modelos similares, serão apresentados ao longo do trabalho. Particularmente, no problema desta seção, o objetivo foi demonstrar uma maior rigidez na região do mecanismo na qual pode ocorrer a instabilidade de tabuleiro, caso o filtro permaneça desativado durante o processo de otimização.

É possível estabelecer uma analogia entre os filtros de imagem, tais como os que são usados para regularizar as topologias dos problemas de otimização, e os filtros de freqüência. Nesta analogia, os gradientes das variáveis de projeto, os quais determinam a escala de transição na passagem entre material base e não-material, correspondem à freqüência. Por assim ser, os filtros espaciais de densidades são classificados como filtros "passa-baixa" (CARDOSO; FONSECA, 2003), pois apresentam o efeito de limitar estes gradientes a valores máximos. Em termos práticos, os filtros espaciais são uma restrição adicional à formulação do problema de otimização. Com o aumento do raio de abrangência, os gradientes ficam restritos a valores menores, logo, a transição entre material base e não-material se torna mais suave, gerando maiores escalas de cinza. A formulação (2.57) é sensível em relação à escolha do raio de abrangência, ou seja, quando este assume um valor suficientemente grande, os gradientes das variáveis de projeto podem ficar restritos a taxas de variações tão pequenas tais que o problema de otimização pode não ter solução, ao menos para a estimativa inicial adotada. Os próximos resultados demonstram este comportamento. Assim, ao se alterar valor do raio de abrangência de 1,05 mm para 1,30 mm, encontra-se uma nova topologia para o problema de projeto de mecanismo flexível definido na seção 4.2.3, vide Figura 4.26.

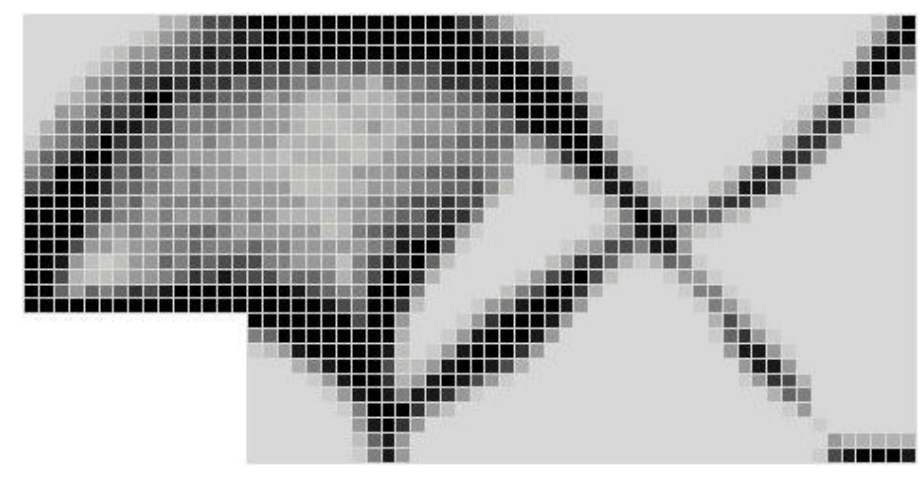

Figura 4.26 - Topologia obtida com $\mathrm{R}_{\max }=1,30 \mathrm{~mm}$. 

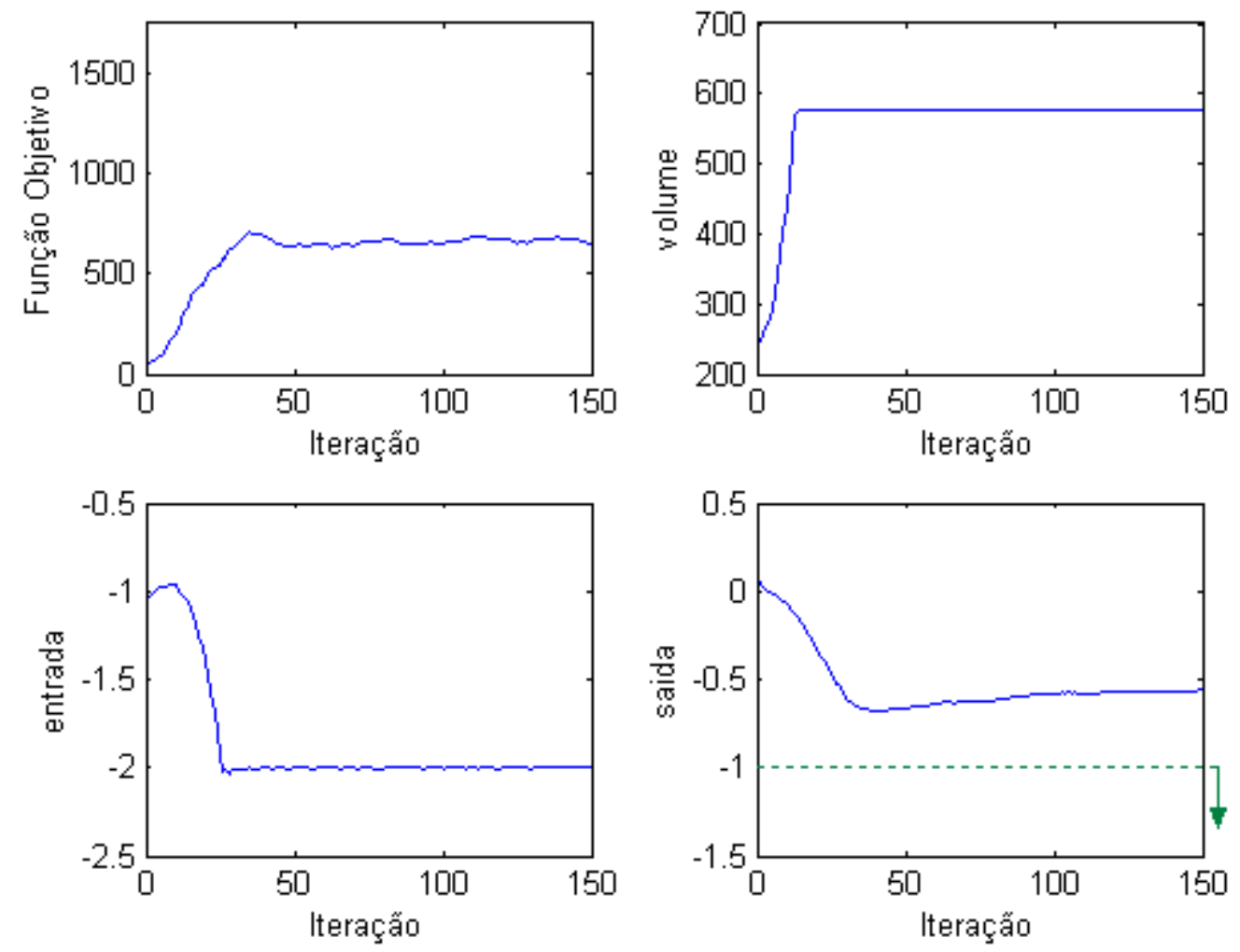

Figura 4.27 - Gráficos de convergência referentes à topologia obtida com $\mathrm{R}_{\max }=1,30 \mathrm{~mm}$.

Na topologia mostrada pela Figura 4.26 o deslocamento do ponto de saída não atendeu à restrição relacionada à compressão da mola, conforme se constata através do gráfico de saída da Figura 4.27. A seguir, tem-se uma nova topologia obtida a partir do mesmo problema. Neste último caso, considerou-se um raio de abrangência ainda maior 1,60 mm.

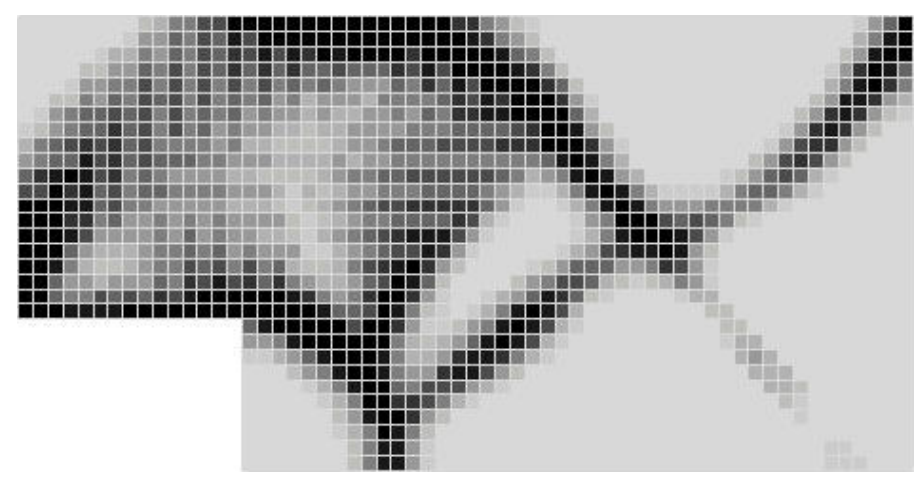

Figura 4.28 - Topologia obtida com $\mathrm{R}_{\max }=1,60 \mathrm{~mm}$. 

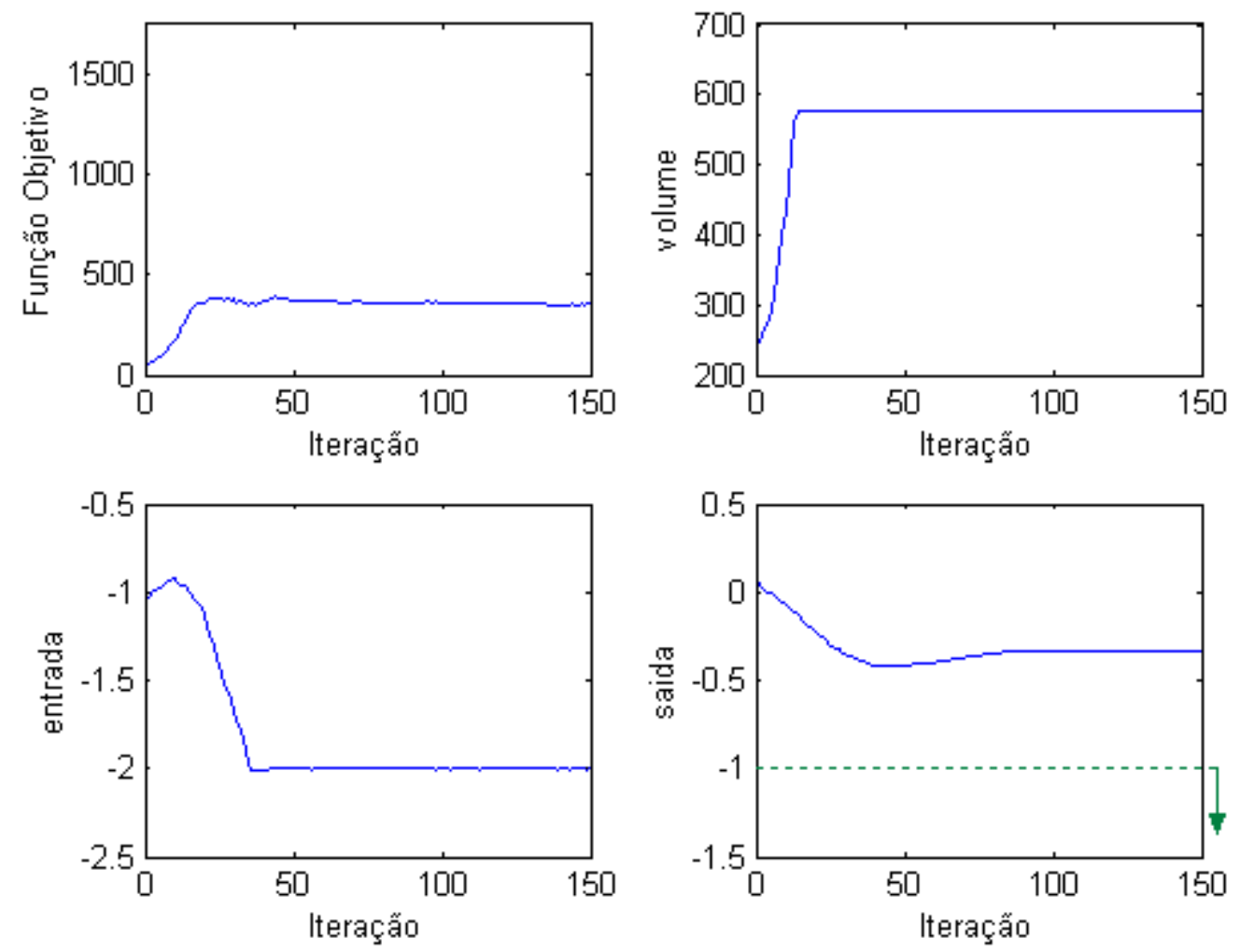

Figura 4.29 - Gráficos de convergência referentes à topologia obtida com $\mathrm{R}_{\max }=1,60 \mathrm{~mm}$.

De acordo com o esperado, devido ao incremento no raio de abrangência, houve um aumento das escalas de cinza, fazendo com que o resultado da Figura 4.28 esteja mais “distante” das especificações de projeto, o que pode ser observado por meio dos gráficos de convergência presentes na Figura 4.29.

\subsection{ASPECTOS CARACTERÍSTICOS DAS TOPOLOGIAS}

\subsubsection{Comentários iniciais}

Neste capítulo, a redução das “dobradiças” nas topologias dos mecanismos flexíveis projetados por meio da formulação (2.57) é demonstrada. Outra particularidade que a formulação oferece é a possibilidade de controlar os deslocamentos, não só dos pontos de entrada e saída de força, mas também de qualquer outro ponto que seja de interesse do 
projetista. Para tanto, é necessário impor restrições no deslocamento. É importante deixar claro que a solução do problema de otimização pode não existir, caso sejam adotados valores inadequados em relação a estas restrições, que integram, juntamente com os demais parâmetros da otimização (carregamento, geometria do domínio fixo, rigidez da mola, propriedades elásticas do material base e restrição volumétrica), a relação de dados que definem o problema de projeto.

Na seção 4.4.2, como forma de ilustrar um resultado isento de “dobradiças”, faz-se para um mesmo problema de otimização - a comparação entre a topologia obtida através da maximização da energia de deformação, conforme sugere Cardoso e Fonseca (2004), e a topologia determinada pela maximização da energia mútua e minimização da flexibilidade média, segundo propõem Nishiwaki et al. (1998). Ainda na seção 4.4.2, investigam-se as tensões internas presentes nos mecanismos flexíveis submetidos aos carregamentos para o quais foram projetados. As alterações de topologia decorrentes da imposição de novas restrições de deslocamento são demonstradas na seção 4.4.3.

\subsubsection{Redução da ocorrência de “dobradiças”}

Para ilustrar a redução da ocorrência de “dobradiças” nas topologias obtidas através da formulação (2.57), utilizou-se, como exemplo, o problema de projeto de mecanismo flexível representado pela Figura 4.30 .

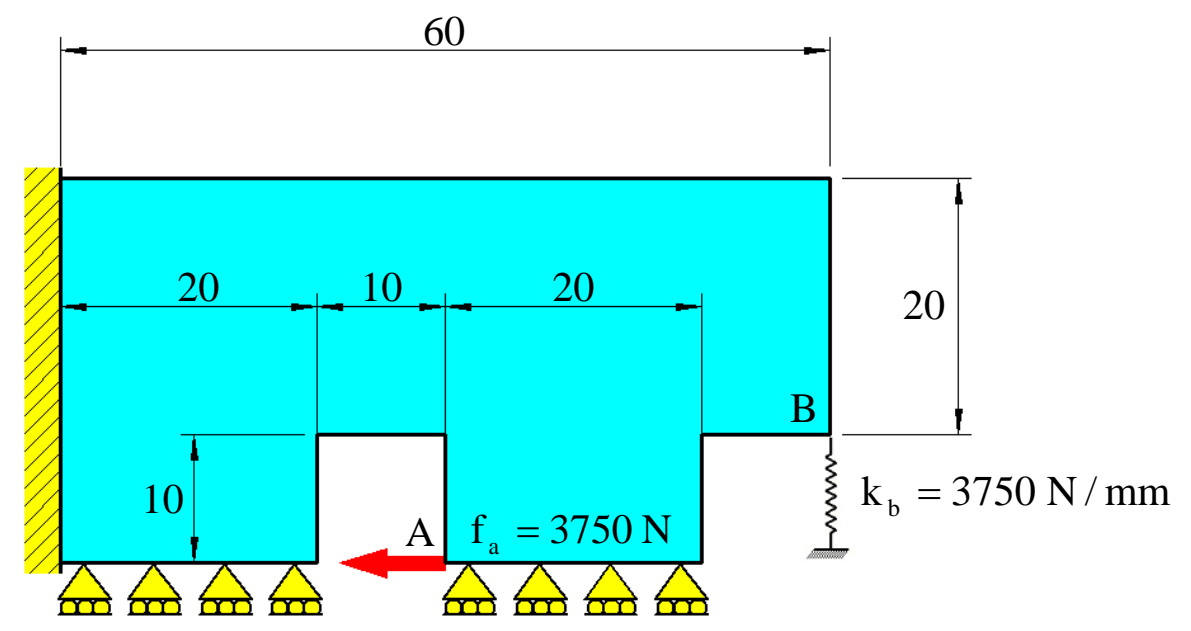

Figura 4.30 - Representação do problema de otimização. 
A simetria do problema é considerada na definição da geometria do domínio fixo de projeto demonstrado na Figura 4.30. O aço é o material base do qual o mecanismo será constituído. A força representada por $\mathrm{f}_{\mathrm{a}}$ provocará um deslocamento máximo de $2 \mathrm{~mm}$ no seu ponto de aplicação, além de um deslocamento mínimo de $1 \mathrm{~mm}$ na compressão da mola de rigidez $\mathrm{k}_{\mathrm{b}}$. Os valores destes parâmetros estão indicados na Figura 4.30. Para a solução do problema, restringiu-se o material do mecanismo em 20\% do volume do domínio fixo, o qual foi discretizado em 2304 elementos finitos. Iniciou-se o processo de otimização a partir de 0,15 , valor este adotado como estimativa inicial atribuída a todas as variáveis de projeto. $\mathrm{O}$ raio de abrangência considerado no esquema de filtragem foi de 0,9 mm. A topologia da Figura 4.31 corresponde à solução encontrada por meio da formulação (2.57).

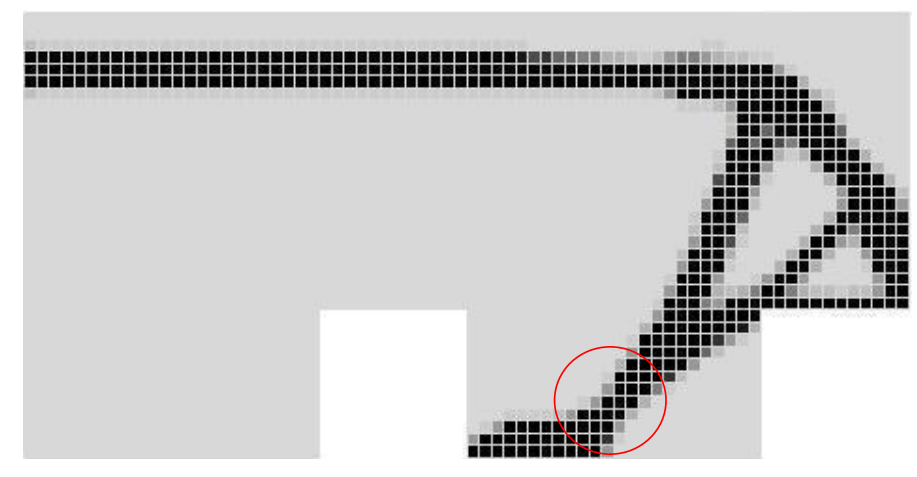

Figura 4.31 - Topologia obtida por meio da formulação (2.57).

O mesmo problema proposto nesta seção foi solucionado com a aplicação de um código computacional - elaborado por Lima (2002) - cuja função multi-objetivo implementada promove a maximização da energia mútua e a minimização da flexibilidade (NISHIWAKI et al., 1998). O resultado pode ser visto na Figura 4.32.

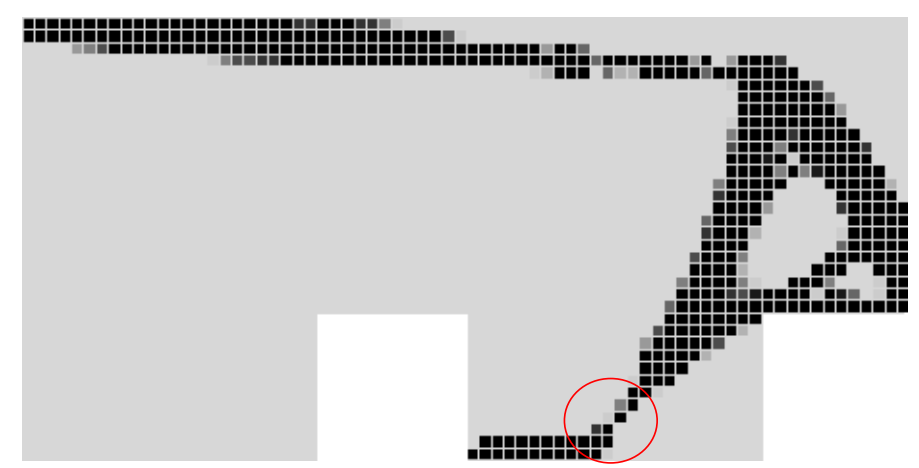

Figura 4.32 - Topologia obtida por meio do código computacional elaborado por Lima (2002). 
O código computacional empregado para obter a imagem da Figura 4.32 incorpora o mesmo filtro espacial de densidades implementado conjuntamente com a formulação (2.57). Contudo, a imagem da Figura 4.31 não apresenta a “dobradiça”, conforme se constata na comparação entre as regiões destacadas nas duas figuras. A razão pela qual isso ocorre é que, no caso mostrado pela Figura 4.31, o otimizador disponibiliza apenas a energia necessária à deformação da mola tal que a restrição de deslocamento correspondente seja atendida. Já na topologia da Figura 4.32, toda a energia possível será fornecida pelo mecanismo, e como foi discutido na seção 2.5, este comportamento favorece a formação das “dobradiças”.

Interpretando a solução apontada pela Figura 4.31 foi possível chegar à configuração do mecanismo flexível cuja deformação, quando submetido ao carregamento para o qual foi projetado, está demonstrada na Figura 4.33.

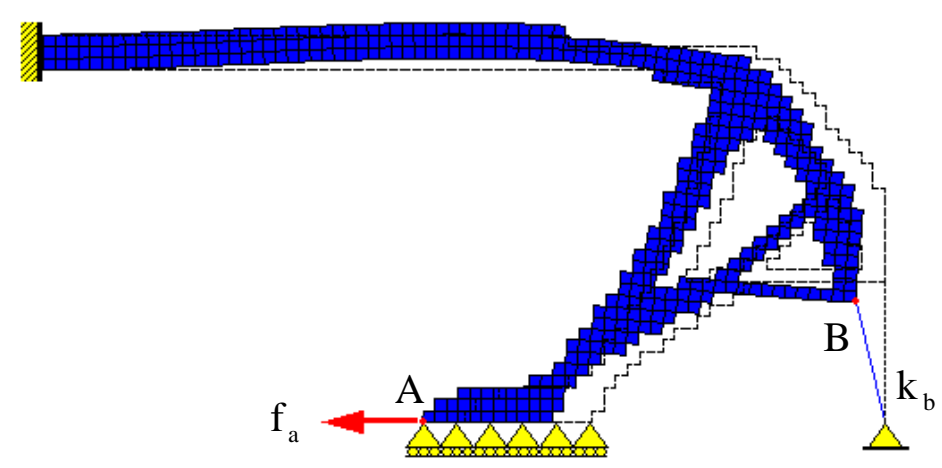

Figura 4.33 - Configuração deformada.

Tabela 4.1 - Comparação entre os deslocamentos observados nas diferentes etapas de projeto.

\begin{tabular}{lccc}
\hline Grau de liberdade & $\begin{array}{c}\text { Restrição imposta ao } \\
\text { deslocamento }\end{array}$ & $\begin{array}{c}\text { Deslocamento no final do } \\
\text { processo de OT }\end{array}$ & $\begin{array}{c}\text { Deslocamento obtido na } \\
\text { análise do modelo de EF }\end{array}$ \\
\hline A horizontal & $-2,00 \mathrm{~mm}$ (valor mínimo) & $-2,00 \mathrm{~mm}$ & $-2,33 \mathrm{~mm}$ \\
B vertical & $-1,00 \mathrm{~mm}$ (valor máximo) & $-1,00 \mathrm{~mm}$ & $-1,10 \mathrm{~mm}$ \\
\hline
\end{tabular}

A Tabela 4.1 indica os deslocamentos dos pontos A e B ao final de cada uma das duas etapas de projeto - otimização e análise estática do modelo construído a partir da interpretação da solução. A diferença de valores na comparação entre as etapas decorre da interpretação, conforme já foi discutido na seção 2.3.1. Em relação às tensões internas, a análise sobre o modelo fornece o mapa de cores da Figura 4.34, que, devido à alta rigidez $\mathrm{k}_{\mathrm{b}}$, apresenta tensões muito elevadas segundo os padrões mais comuns da Engenharia. Para contornar os valores extremos das tensões mecânicas, é necessário considerar a aplicação e a reação de forças distribuídas. Desta forma, o problema de projeto de mecanismo flexível utilizado nesta seção fica redefinido de acordo com a Figura 4.35. 


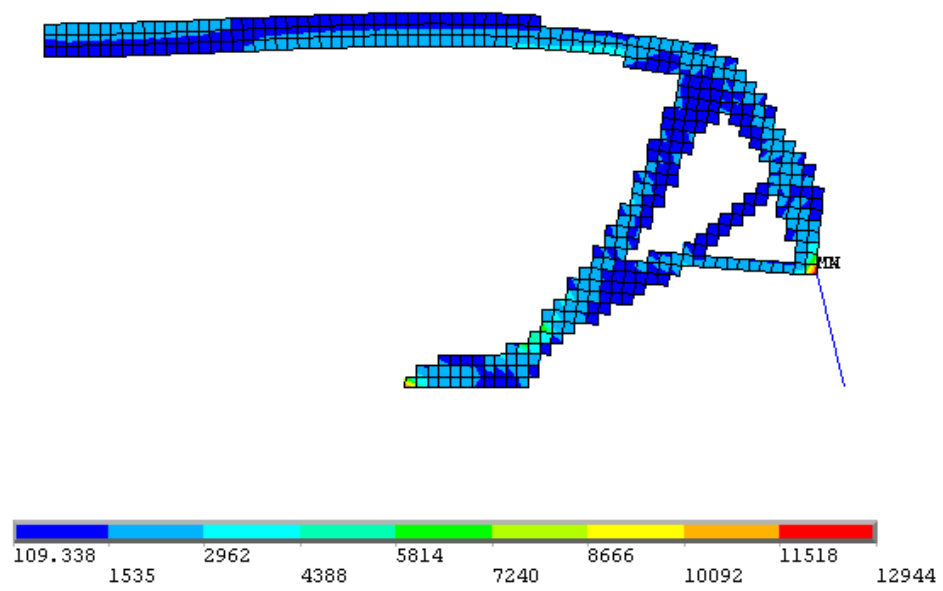

Figura 4.34 - Mapa de cores representando as tensões segundo o critério de resistência de von Mises (em $\left.\mathrm{N} / \mathrm{mm}^{2}\right)$.

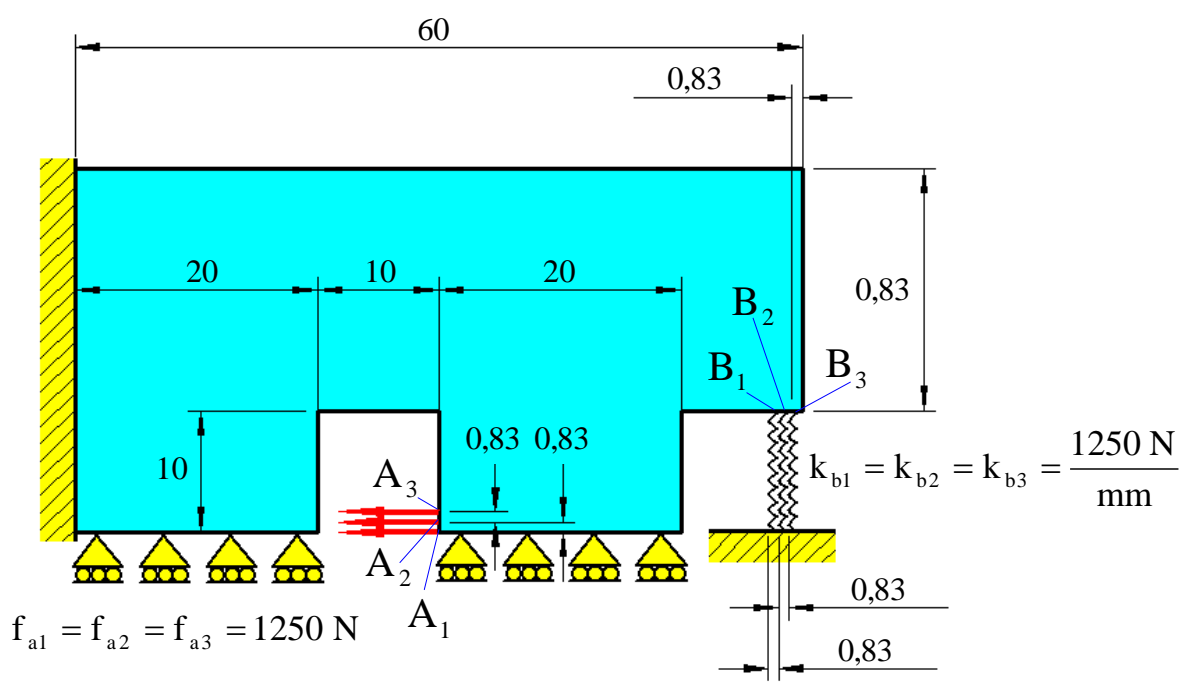

Figura 4.35 - Representação do problema de otimização.

A Figura 4.36 apresenta a solução do problema representado pela Figura 4.35.

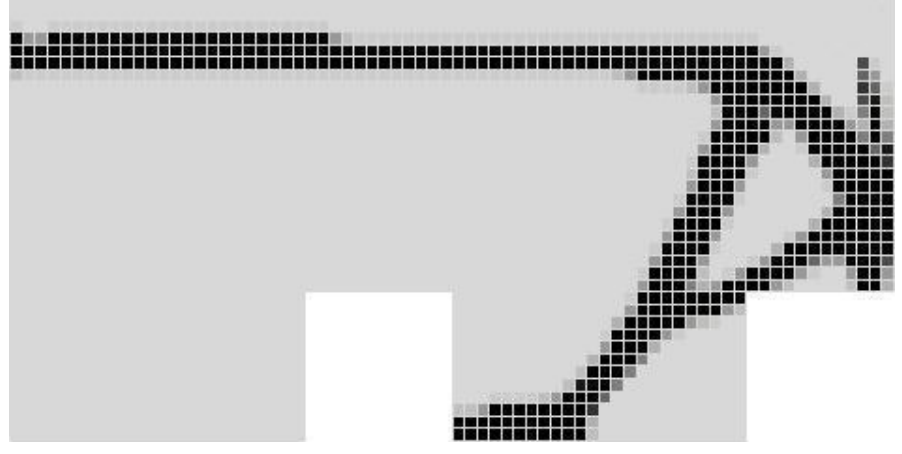

Figura 4.36 - Topologia obtida para o problema que considera as forças distribuídas. 
Interpretando a topologia indicada pela Figura 4.36 foi possível chegar à configuração do mecanismo flexível cujas tensões mecânicas, quando submetido ao carregamento para o qual foi projetado, estão no mapa de cores da Figura 4.37.

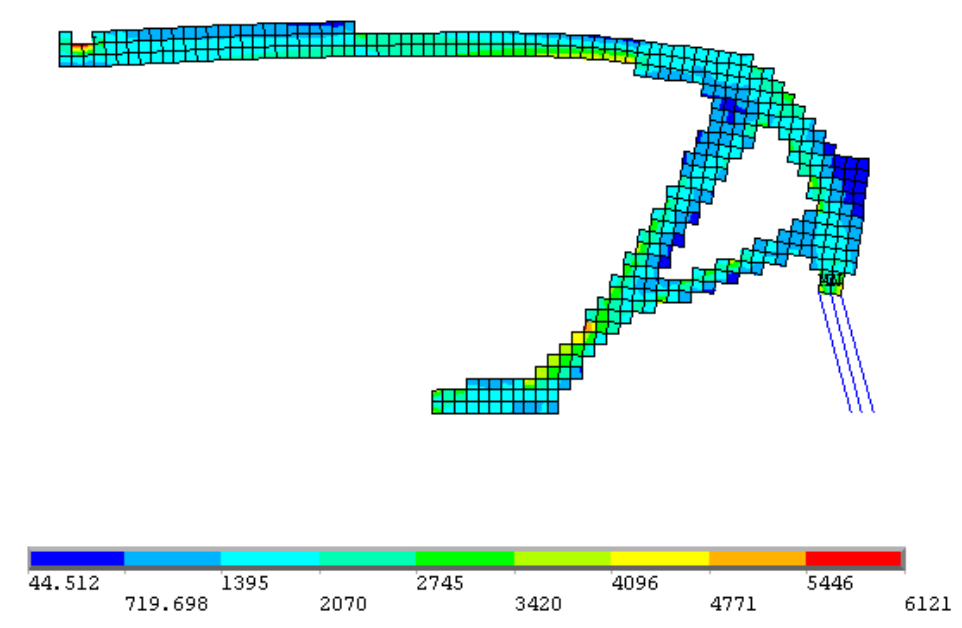

Figura 4.37 - Mapa de cores representando as tensões segundo o critério de resistência de von Mises (em $\left.\mathrm{N} / \mathrm{mm}^{2}\right)$.

Vê-se claramente que a consideração de forças distribuídas reduziu o valor máximo da tensão imposta ao dispositivo. É uma constatação bastante importante, e até intuitiva, visto que a redefinição proposta para o problema determina um modelo mais próximo da realidade.

\subsubsection{Comportamento cinemático do mecanismo flexível}

A análise estática do modelo construído a partir da interpretação da topologia que é demonstrada pela Figura 4.36 também nos fornece a configuração deformada do mecanismo, bem como os valores dos deslocamentos causados pela aplicação do carregamento, vide Figura 4.38 e Tabela 4.2. 


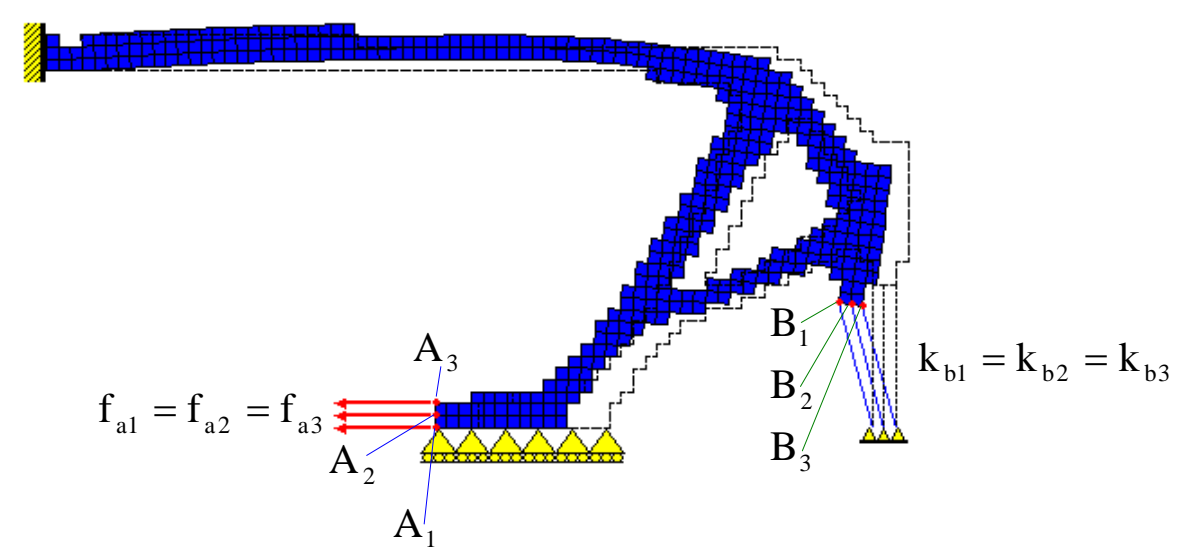

Figura 4.38 - Configuração deformada.

Tabela 4.2 - Comparação entre os deslocamentos observados nas diferentes etapas de projeto.

\begin{tabular}{lccc}
\hline Grau de liberdade & $\begin{array}{c}\text { Restrição imposta ao } \\
\text { deslocamento }\end{array}$ & $\begin{array}{c}\text { Deslocamento no final do } \\
\text { processo de OT }\end{array}$ & $\begin{array}{c}\text { Deslocamento obtido na } \\
\text { análise do modelo de EF }\end{array}$ \\
\hline A1 horizontal & $-2,00 \mathrm{~mm}$ (valor mínimo) & $-2,00 \mathrm{~mm}$ & $-2,51 \mathrm{~mm}$ \\
A2 horizontal & $-2,00 \mathrm{~mm}$ (valor mínimo) & $-1,99 \mathrm{~mm}$ & $-2,50 \mathrm{~mm}$ \\
A3 horizontal & $-2,00 \mathrm{~mm}$ (valor mínimo) & $-2,00 \mathrm{~mm}$ & $-2,51 \mathrm{~mm}$ \\
B $_{1}$ horizontal & Não especificada & Não observado & $-1,97 \mathrm{~mm}$ \\
B2 horizontal & Não especificada & Não observado & $-1,96 \mathrm{~mm}$ \\
B3 horizontal & Não especificada & Não observado & $-1,96 \mathrm{~mm}$ \\
B 1 vertical & $-1,00 \mathrm{~mm}$ (valor máximo) & $-1,00 \mathrm{~mm}$ & $-1,08 \mathrm{~mm}$ \\
B2 vertical & $-1,00 \mathrm{~mm}$ (valor máximo) & $-1,03 \mathrm{~mm}$ & $-1,17 \mathrm{~mm}$ \\
B3 vertical & $-1,00 \mathrm{~mm}$ (valor máximo) & $-1,02 \mathrm{~mm}$ & $-1,23 \mathrm{~mm}$ \\
\hline
\end{tabular}

Cada um dos pontos $\mathrm{B}_{1}, \mathrm{~B}_{2}$ e $\mathrm{B}_{3}$ apresenta um deslocamento horizontal para o qual o problema definido não faz menção. Pode-se supor, por alguma razão inerente ao projeto, que estes deslocamentos horizontais são indesejados, o que corresponderia a um novo comportamento cinemático, caso fossem anulados. Com o propósito de obter um mecanismo flexível com esta característica, foram introduzidas novas restrições ao deslocamento dos pontos em questão. Supondo que $m, m+2$ e $m+4$ sejam, respectivamente, os graus de liberdade globais associados aos deslocamentos horizontais dos pontos $B_{1}$, $B_{2}$ e $B_{3}$, então, consideram-se as restrições $u_{g_{m}} \geq-0,05, u_{g_{m+2}} \geq-0,05$ e $u_{g_{m+4}} \geq-0,05$ na definição do problema de otimização. Com a adoção do sistema usual de coordenadas globais - sistema (x,y) demonstrado pela Figura 2.1 -, os pontos de saída de força da nova topologia não devem se deslocar além de $0,05 \mathrm{~mm}$ à esquerda. Porém, o deslocamento à direita também deve ser restringido. Para tanto, adotam-se as restrições: $\mathrm{u}_{\mathrm{g}_{\mathrm{m}}} \leq 0,05 ; \mathrm{u}_{\mathrm{g}_{\mathrm{m}+2}} \leq 0,05 ; \mathrm{u}_{\mathrm{g}_{\mathrm{m}+4}} \leq 0,05$. Assim, através da utilização destas seis inequações, foi possível obter a topologia mostrada pela Figura 4.39. 
119

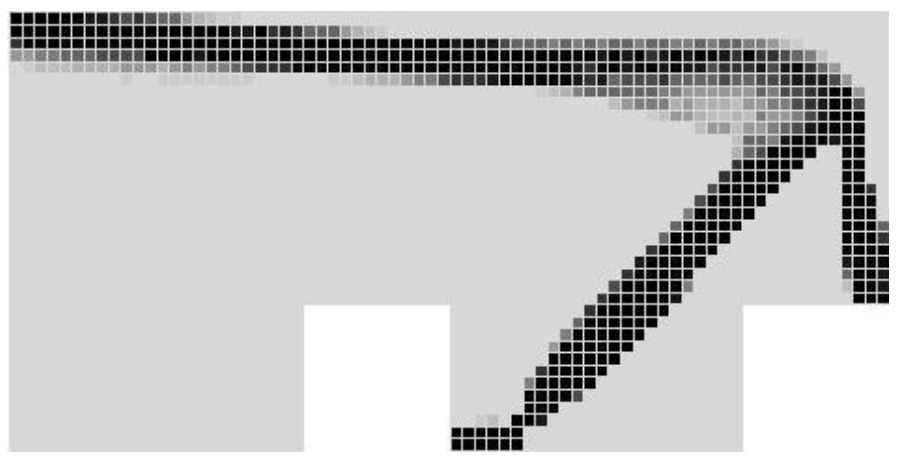

Figura 4.39 - Topologia obtida para o problema cujos deslocamentos horizontais dos pontos de saída foram restringidos.

A análise estática do modelo construído a partir da interpretação da topologia que é demonstrada pela Figura 4.39 fornece a configuração deformada do mecanismo, bem como os deslocamentos causados pelo carregamento aplicado, vide Figura 4.40 e Tabela 4.3.

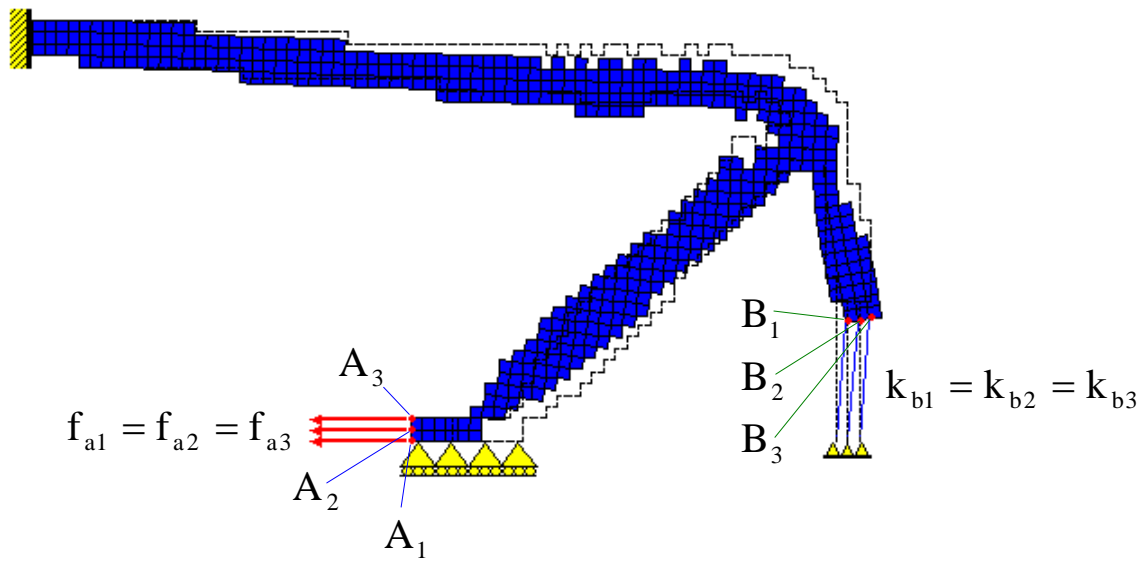

Figura 4.40 - Configuração deformada.

Tabela 4.3 - Comparação entre os deslocamentos observados nas diferentes etapas de projeto.

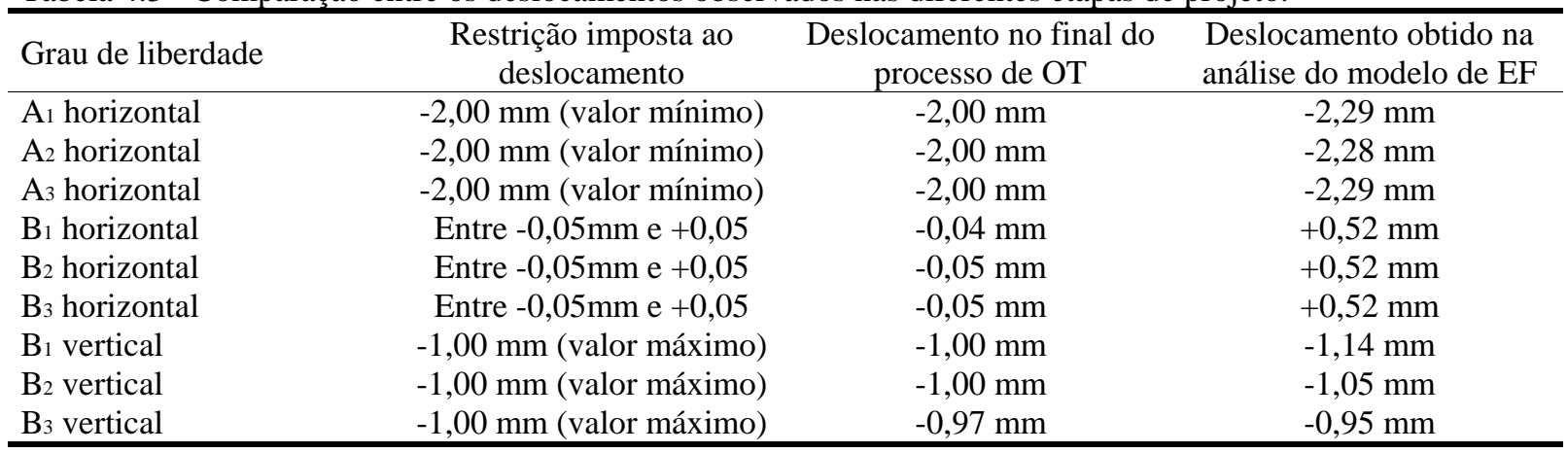

Os pontos $\mathrm{B}_{1}, \mathrm{~B}_{2}$ e $\mathrm{B}_{3}$ do mecanismo projetado ainda apresentam deslocamentos horizontais indesejáveis, apesar de serem sensivelmente menores. A razão pela qual estes deslocamentos ainda persistem está relacionada aos erros inerentes à interpretação feita sobre 
a solução do problema de otimização topológica. Certamente, seria necessário estabelecer um critério mais rigoroso para interpretar o resultado de forma mais eficiente, considerando, inclusive, o refinamento da malha de EF. Contudo, o exercício de introduzir as restrições de deslocamento ilustrou o recurso que a formulação (2.57) oferece em relação à definição do comportamento cinemático do dispositivo. Dada a simetria do problema, apenas a sua metade foi considerada no processo de otimização, assim, a configuração final do mecanismo flexível completo está demonstrada na Figura 4.41.

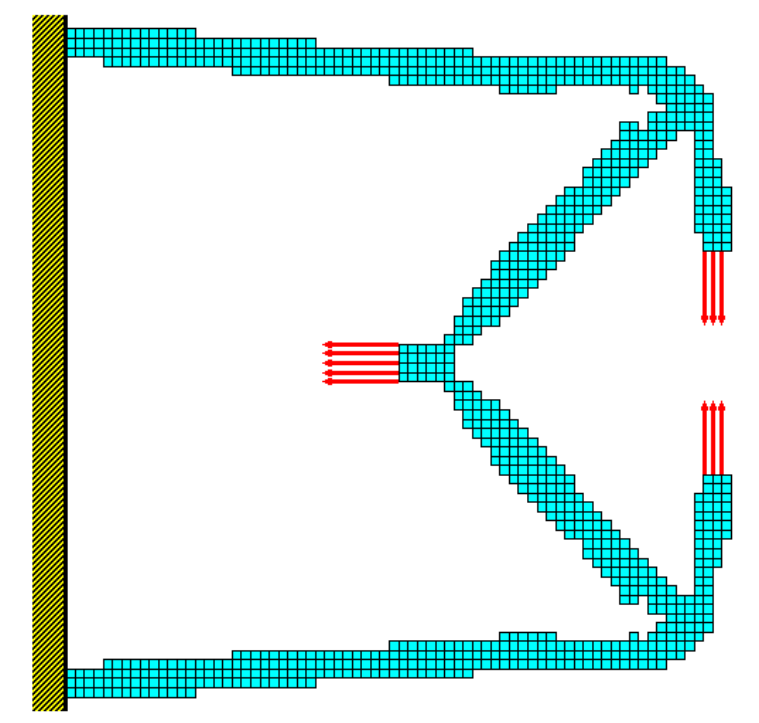

Figura 4.41 - Configuração final do mecanismo flexível.

\subsection{AVALIAÇÃO DO EFEITO DA VARIAÇÃO DO RAIO DO FILTRO E DO VOLUME RESTRINGIDO}

\subsubsection{Comentários iniciais}

Entender a influência que estes parâmetros - a restrição volumétrica e o raio de abrangência do filtro espacial de densidades - exercem sobre a topologia é algo muito útil para reforçar a compreensão de alguns aspectos acerca da formulação discutida na seção 2.6.4. Entender a relação que há entre a quantidade de material empregado e a forma pela qual a energia de deformação se distribui no interior do mecanismo corrobora com a idéia de que a 
formulação ajusta a topologia à diferença entre o trabalho disponibilizado ao mecanismo e o trabalho fornecido pelo mecanismo. Esta diferença, conforme se sabe, corresponde exatamente à energia de deformação armazenada no dispositivo. Assim, na seção 4.5.2, adotase um problema de projeto para ilustrar os efeitos causados à topologia quando alteramos a quantidade de material disponível para otimização. Na seção 4.5.3, discute-se uma forma alternativa de utilização do filtro espacial de densidades de modo que seja possível reduzir as escalas de cinza cuja extensão possa comprometer a representação das regiões estreitas das topologias, e, como será visto na seção 4.5.2, as regiões com este aspecto podem surgir em função do volume de material empregado no processo de otimização. A seção 4.5.4 apresenta os efeitos decorrentes do refinamento da malha adotado conjuntamente com a redução do raio de abrangência do esquema de filtragem.

\subsubsection{Variação da restrição do volume de material}

A Figura 4.42 representa o problema de projeto de mecanismo flexível utilizado para ilustrar a influência que a variação da restrição do volume de material exerce sobre a topologia.

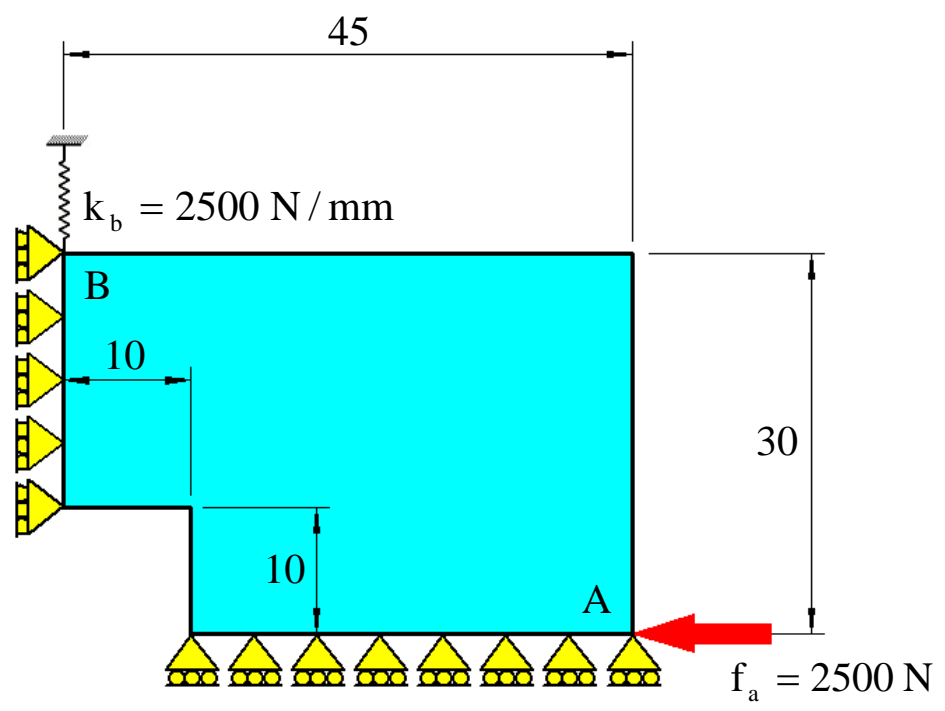

Figura 4.42 - Representação do problema de otimização. 
O aço é o material base do qual o mecanismo será constituído. A força representada por $\mathrm{f}_{\mathrm{a}}$ provocará um deslocamento máximo de $2 \mathrm{~mm}$ no seu ponto de aplicação, além de um deslocamento mínimo de $1 \mathrm{~mm}$ na compressão da mola de rigidez $\mathrm{k}_{\mathrm{b}}$. Os valores destes parâmetros estão indicados na Figura 4.42. A fim de solucionar o problema, o domínio fixo de projeto foi discretizado em 3494 elementos finitos. Iniciou-se o processo de otimização a partir de 0,15, valor este adotado como estimativa inicial atribuída a todas as variáveis de projeto. O raio de abrangência considerado para o esquema de filtragem foi de 0,85 mm. As Figuras 4.43, 4.44 e 4.45 correspondem às topologias obtidas com base nas restrições de 15\%, $20 \%$ e $25 \%$ do volume do domínio fixo, respectivamente.

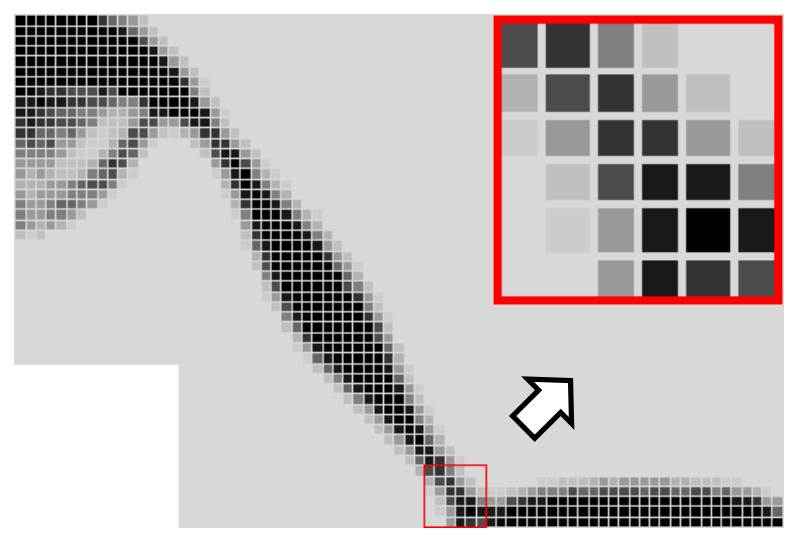

Figura 4.43 - Topologia obtida com restrição de 15\% do volume do domínio fixo.

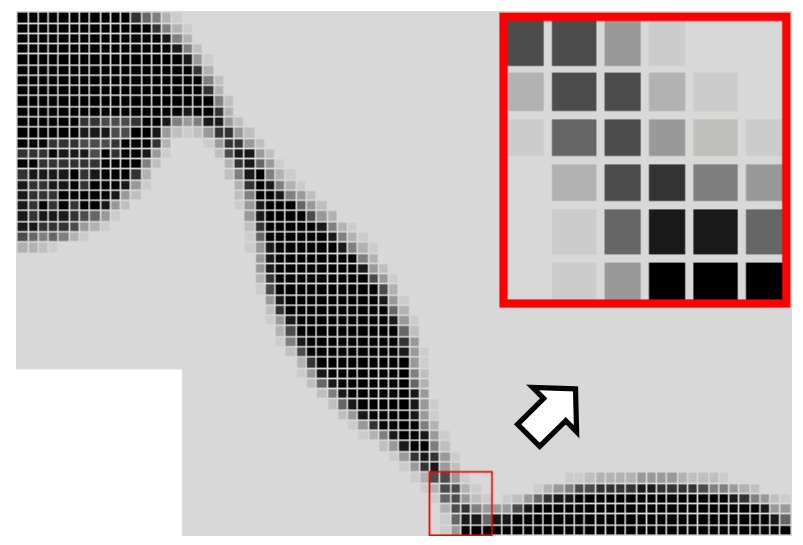

Figura 4.44 - Topologia obtida com restrição de 20\% do volume do domínio fixo. 


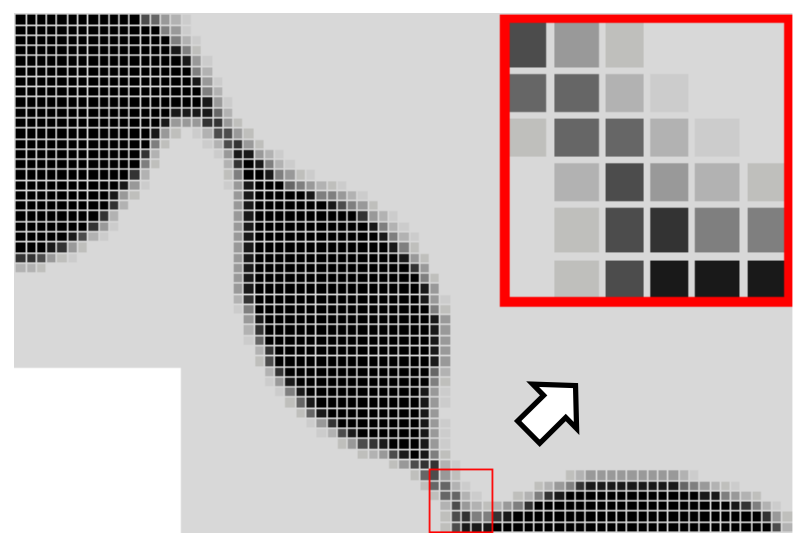

Figura 4.45 - Topologia obtida com restrição de 25\% do volume do domínio fixo.

Verificou-se que as restrições de deslocamento ficaram ativas nos três processos de otimização. Deduz-se então, que cada uma das três topologias absorveu a mesma quantidade de energia de deformação. Contudo, nas topologias mais volumosas, observam-se áreas nas quais a energia se concentra. Esta constatação é feita visualmente, e pode ser ilustrada por meio das regiões destacadas nas Figuras 4.43, 4.44 e 4.45. Cada uma destas áreas em destaque apresenta, em seu núcleo, uma densidade menor na medida em que a topologia correspondente contém mais material. Como se sabe, regiões menos densas submetidas às mesmas tensões internas absorvem mais energia de deformação. Logo, com o aumento do material disponível à síntese do mecanismo, as regiões em destaque surgem como áreas nas quais a flexibilidade do mecanismo se concentra. A explicação para o comportamento está relacionada aos “braços” mais robustos e rígidos presentes nas topologias de maior volume. Nestas condições, para que a topologia absorva a mesma quantidade de energia que foi armazenada nos casos para os quais a demanda de material foi menor, ela precisa concentrar esta quantidade de energia em áreas específicas, normalmente, naquelas que servem de união entre os "braços". Isso, em parte, explica a presença inconveniente de zonas de baixa densidade no núcleo das regiões estreitas existentes nas topologias mais volumosas. No entanto, a análise não deve contemplar apenas este aspecto, os resultados sugerem que o filtro espacial de densidades também tem influência fundamental, pois em última análise, ele não deixa de ser uma espécie de restrição adicional ao problema de otimização, forçando para que haja a distribuição suave de material pela topologia do mecanismo. Para atender esta condição, as escalas de cinza geradas devido ao filtro podem impedir a presença de material base no núcleo das regiões mais estreitas, caso estas não sejam suficientemente largas para permitir a transição completa entre não-material e material base. Uma solução com respeito ao problema é o refinamento da malha de EF acompanhado da redução do raio de abrangência 
do filtro. Este expediente será demonstrado mais adiante. No entanto, para evitar os custos computacionais gerados a partir do refinamento da malha, a próxima seção apresenta uma solução alternativa mais simples e menos custosa.

\subsubsection{Redução das escalas de cinza por meio da desativação do filtro}

Com respeito ao esquema de filtragem, sabe-se que a escolha de um raio de abrangência suficientemente grande produz escalas de cinza cujas extensões podem ser proibitivas à interpretação da solução do problema. Por outro lado, não é recomendável adotar raios de abrangência muito próximos de seus valores mínimos de influência ${ }^{2}$, pois a eficiência do filtro fica comprometida. Um exemplo disso é visto a seguir. No problema da seção 4.5.2, a discretização correspondente a 3494 elementos finitos quadrados implica na distância mínima de 0,60 mm medida entre dois centróides consecutivos da malha. O mesmo problema foi solucionado para uma restrição volumétrica igual a 25\% do volume do domínio fixo, tendo sido considerado, no entanto, o valor de 0,61 mm para o raio de abrangência do esquema de filtragem. Vê-se o resultado da otimização na figura abaixo.

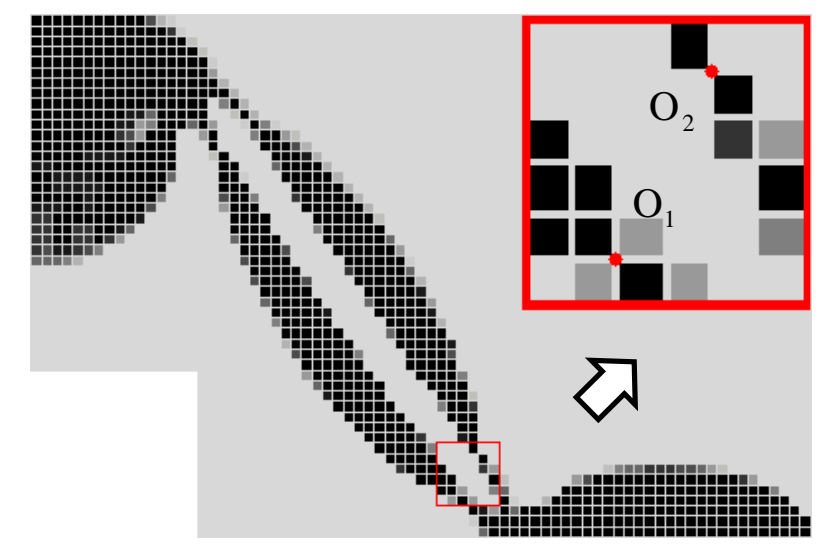

Figura 4.46 - Topologia obtida com um raio de abrangência igual a 0,61 mm.

\footnotetext{
${ }^{2} \mathrm{O}$ menor raio de abrangência possível para o filtro espacial de densidades corresponde à distância entre o centróide do elemento sobre o qual se aplica o filtro e o centróide mais próximo pertencente a um elemento vizinho. Para valores menores do que este, o esquema de filtragem deixa de ser atuante.
} 
Comparando a Figura 4.45 com a Figura 4.46, são observadas diferenças relevantes entre as duas topologias. Estas diferenças decorrem do menor valor adotado para o raio de abrangência relacionado à Figura 4.46. A modificação do raio altera os gradientes das variáveis de projeto e, portanto, outra solução é encontrada. Com a escolha de um valor muito próximo ao mínimo possível $\left(\mathrm{R}_{\max }=0,61 \mathrm{~mm}>0,60 \mathrm{~mm}\right.$ ), surgiram escalas de cinza muito reduzidas, tanto que, nesta nova topologia, foi possível observar a transição entre não-material e material base mesmo para aquelas regiões muito estreitas. Uma destas regiões está em destaque na Figura 4.46. Observa-se que o nó $\mathrm{O}_{1}$ é uma “dobradiça” com densidades intermediárias, ou seja, dos quatro elementos que apresentam o nó em comum, dois deles são constituídos de material base (elementos não vizinhos por meio de arestas), já os outros dois têm densidade inferior a um. Apesar de não ser uma "dobradiça” na sua forma clássica, tal como pode ser considerado o nó $\mathrm{O}_{2}$ - também destacado na Figura 4.46 -, a “dobradiça” com densidades intermediárias apresenta os mesmos inconvenientes em relação à interpretação e à fabricação.

Um procedimento para reduzir a ocorrência das áreas de baixa densidade existentes no núcleo das regiões mais estreitas consiste da desativação do filtro espacial nas últimas iterações do processo de otimização (CARDOSO, 2000). As topologias demonstradas nas próximas figuras são resultantes deste método, o qual foi aplicado ao mesmo problema de projeto de mecanismo flexível proposto na seção 4.5.2. Para a comparação entre os resultados, foram mantidas as mesmas restrições de volume, $15 \%$, $20 \%$ e $25 \%$, bem como o mesmo raio de abrangência para o esquema de filtragem $(0,85 \mathrm{~mm})$. Contudo, para obter cada um dos resultados, desativou-se o filtro espacial de densidades nas 10 (dez) iterações seguintes à convergência apontada pelo processo de otimização.

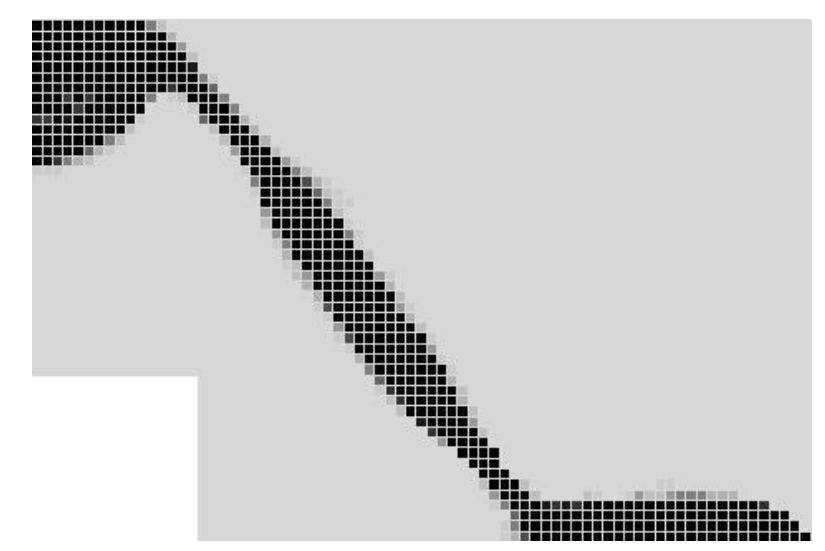

Figura 4.47 - Topologia obtida com restrição de 15\% do volume do domínio fixo. 


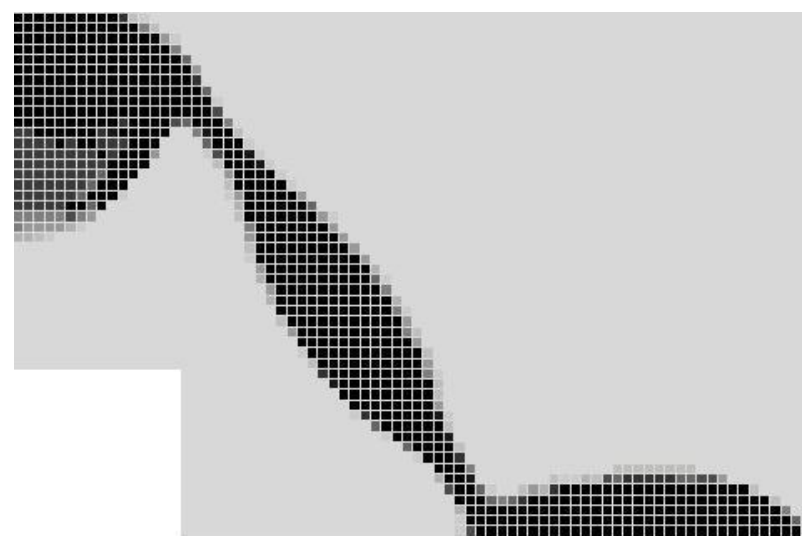

Figura 4.48 - Topologia obtida com restrição de 20\% do volume do domínio fixo.

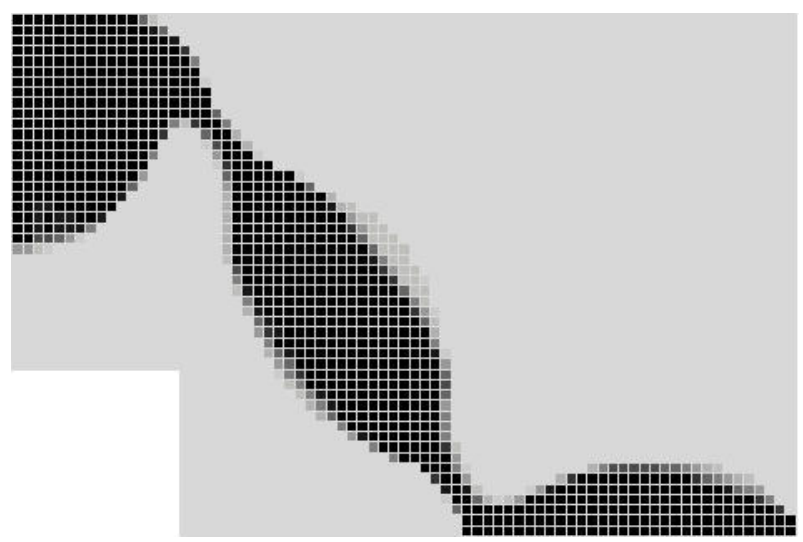

Figura 4.49 - Topologia obtida com restrição de 25\% do volume do domínio fixo.

As Figuras 4.47, 4.48 e 4.49 não apresentam áreas de baixa densidade nas regiões mais “finas” das topologias. As “dobradiças”, por sua vez, foram visivelmente evitadas. Contudo, apesar dos bons resultados alcançados através do método, é necessário que haja um cuidado em relação ao número de iterações efetuadas com o filtro desativado, pois caso muitas delas ocorram antes do término do processo de otimização, a topologia resultante poderá apresentar regiões de instabilidade de tabuleiro.

\subsubsection{Refinamento da malha e redução do raio de abrangência do esquema de filtragem}

Na seção anterior, ficou demonstrado que reduzir o raio de abrangência a valores limites não é um procedimento adequado. Efetuar, arbitrariamente, uma redução menor também não se mostrou apropriado, conforme pode ser visto a seguir. 
Considerando, uma vez mais, o problema da seção 4.5.2 cuja restrição volumétrica corresponde a $25 \%$ do volume do domínio fixo, adotou-se um raio de abrangência menor $(0,70 \mathrm{~mm})$. O resultado da otimização pode ser visto a seguir.

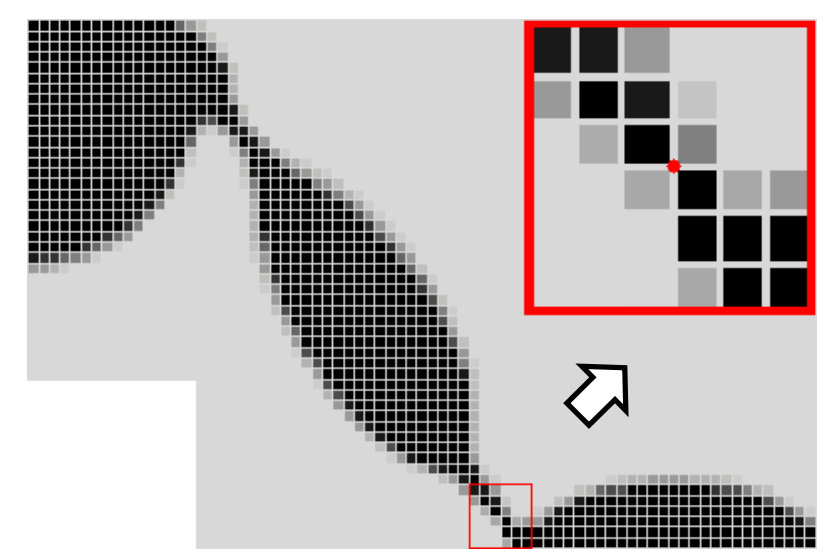

Figura 4.50 - Topologia obtida com um raio de abrangência igual a 0,70 mm.

A Figura 4.50 apresenta uma topologia bastante similar a da Figura 4.45. Contudo, as suas regiões mais "finas” são mais densas do que aquelas observadas na Figura 4.45. A redução do raio de abrangência não foi suficiente para alterar o aspecto da topologia, no entanto, diminuiu a extensão das escalas de cinza. Não obstante ao fato da topologia da Figura 4.50 ser mais bem definida, ainda se observa uma "dobradiça” com densidades intermediárias, conforme se vê na região destacada. Nestas condições, a ação recomendável é o refinamento da malha de EF, através do qual é possível representar melhor a transição de não-material para material base naquelas regiões mais estreitas. As topologias demonstradas nas próximas figuras são resultantes do refinamento da malha aplicado ao domínio fixo do mesmo problema de projeto de mecanismo flexível proposto na seção 4.5.2, tendo sido aumentada a quantidade de 3494 elementos finitos para 4050. A fim de comparar os resultados, foram mantidas as restrições de volume em 15\%, 20\% e 25\%. No entanto, com respeito ao esquema de filtragem, adotou-se o raio de abrangência com o qual a topologia da Figura 4.50 foi obtida, ou seja, $0,70 \mathrm{~mm}$. 


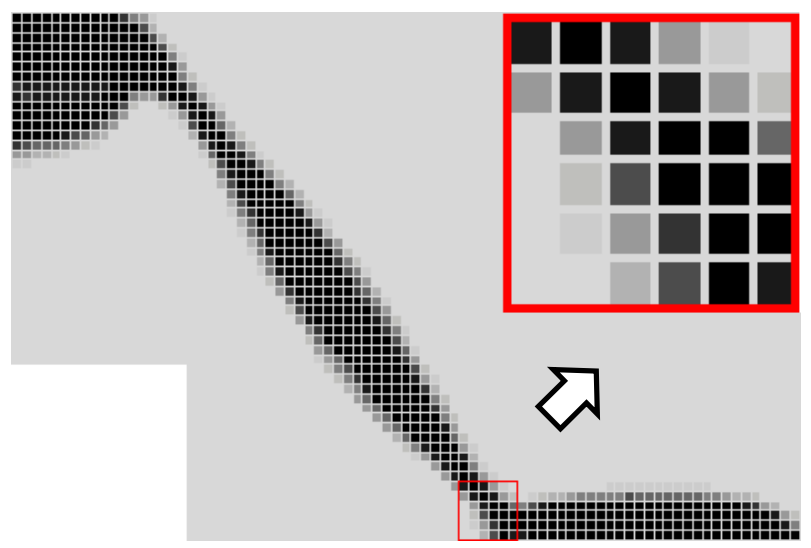

Figura 4.51 - Topologia obtida com restrição de 15\% do volume do domínio fixo.

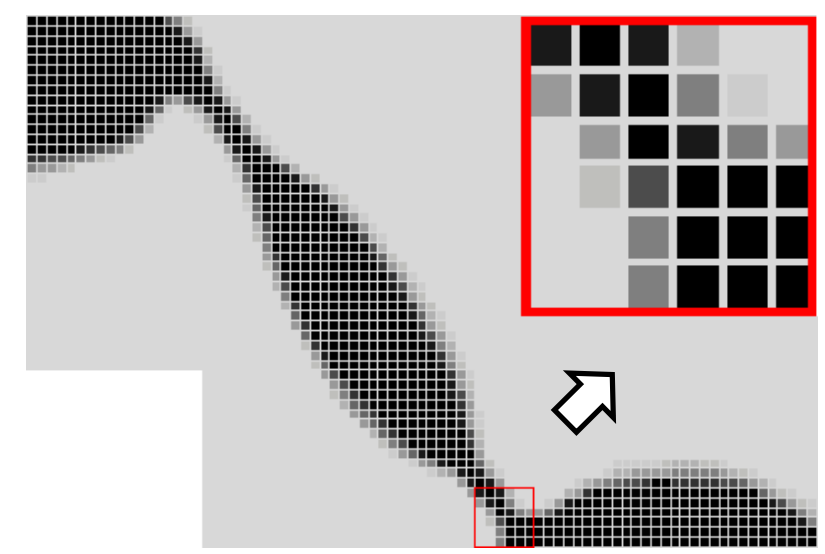

Figura 4.52 - Topologia obtida com restrição de 20\% do volume do domínio fixo.

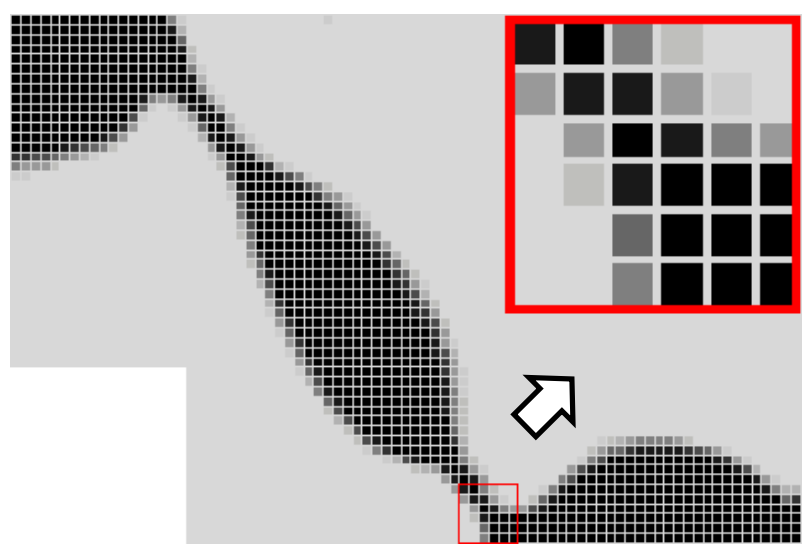

Figura 4.53 - Topologia obtida com restrição de 25\% do volume do domínio fixo.

Os resultados demonstrados não apresentam áreas de baixa densidade no núcleo das regiões mais “finas”, e, principalmente, estão completamente isentos de “dobradiças”. Assim, o tamanho dos elementos finitos ficou adequado ao raio de abrangência do filtro espacial de modo que foi possível representar melhor as regiões mais estreitas das topologias mais 
volumosas. As análises dos modelos criados a partir das interpretações feitas sobre as topologias das Figuras 4.51, 4.52 e 4.53 fornecem as configurações deformadas dos três mecanismos flexíveis e os mapas de cores indicando a distribuição da energia de deformação específica no interior dos dispositivos.

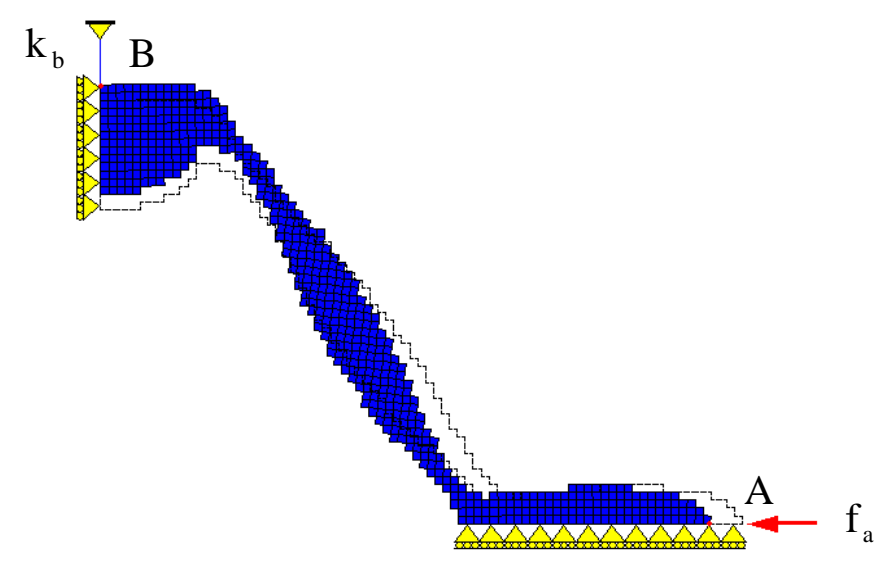

Figura 4.54 - Configuração deformada do mecanismo obtido com restrição volumétrica de 15\%.

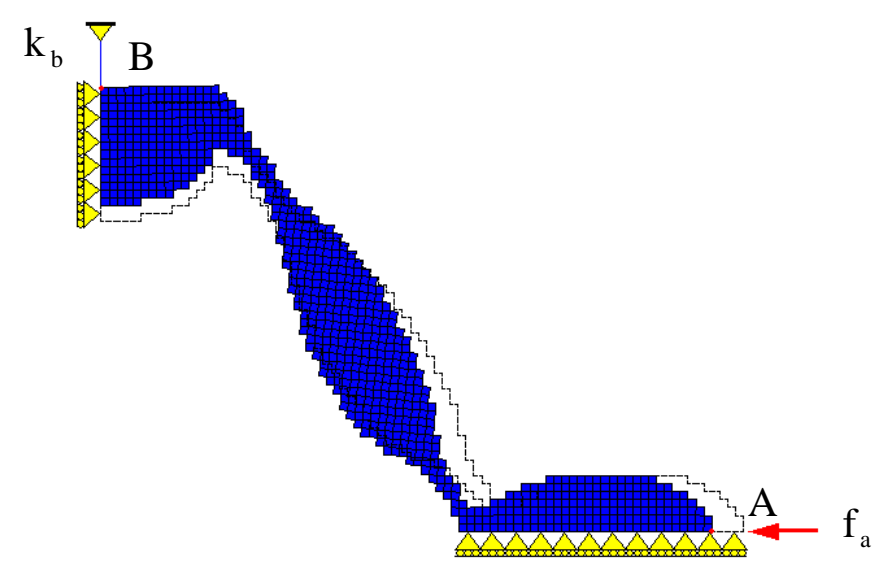

Figura 4.55 - Configuração deformada do mecanismo obtido com restrição volumétrica de 20\%.

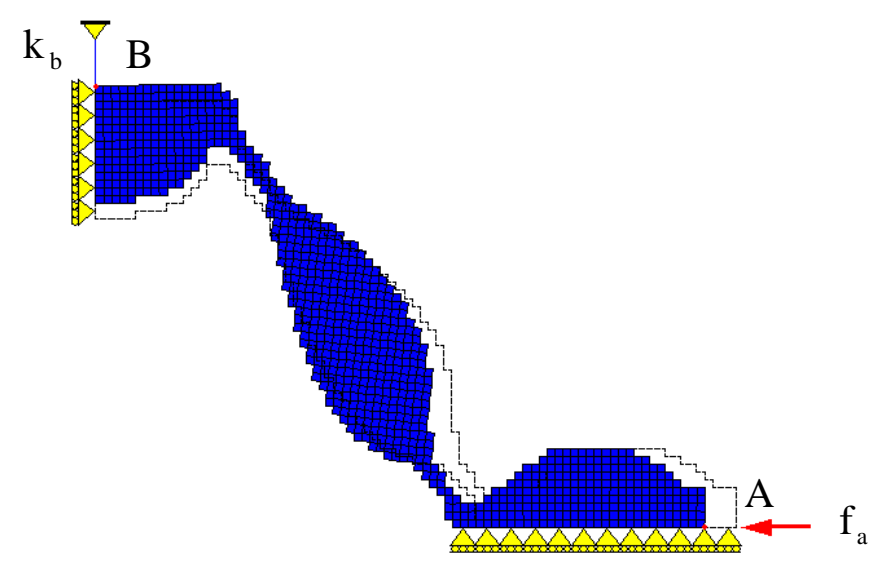

Figura 4.56 - Configuração deformada do mecanismo obtido com restrição volumétrica de 25\%. 


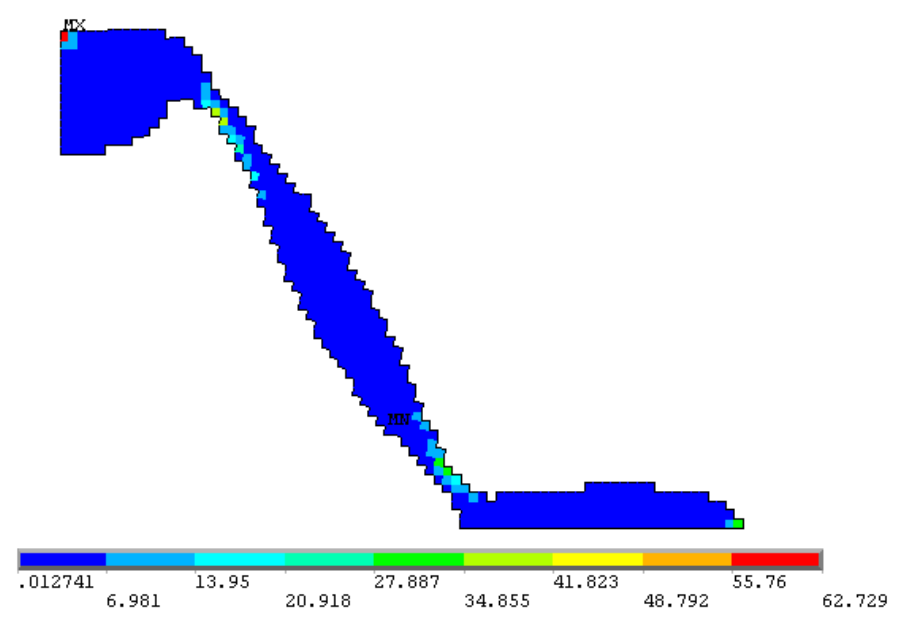

Figura 4.57 - Distribuição da energia de deformação específica (em N/mm²) com restrição volumétrica de 15\%.

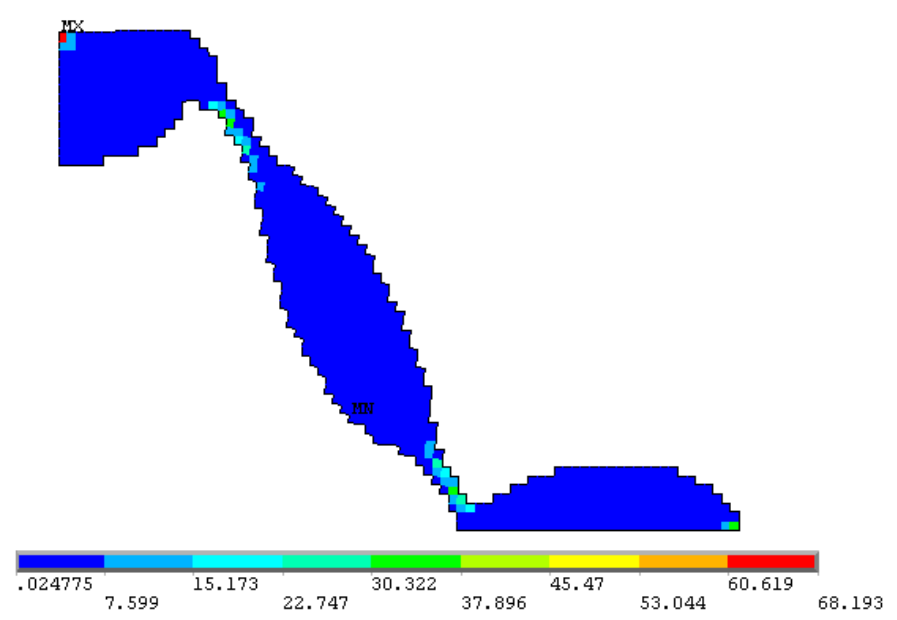

Figura 4.58 - Distribuição da energia de deformação específica (em N/mm²) com restrição volumétrica de 20\%.

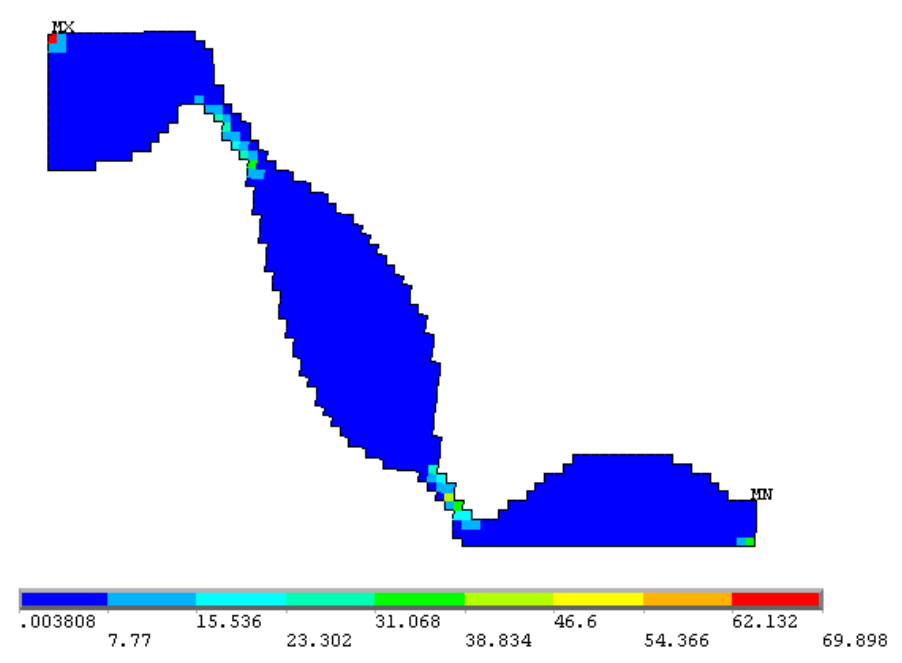

Figura 4.59 - Distribuição da energia de deformação específica (em N/mm²) com restrição volumétrica de 25\%. 
Os mapas das Figuras 4.57, 4.58 e 4.59 corroboram com o conceito descrito na seção 4.5.2, para o qual a energia de deformação tende a se concentrar nas regiões mais “finas”, apresentando valores ainda maiores caso a topologia seja mais volumosa. Cada uma das Tabelas 4.4, 4.5 e 4.6 está relacionada a uma restrição volumétrica distinta empregada no projeto do mecanismo flexível, demonstrando, assim, os deslocamentos e a energia de deformação que foram observados nas duas etapas de projeto - otimização e análise estática do modelo obtido a partir da interpretação feita sobre a solução do problema de otimização.

Tabela 4.4 - Comparação entre os valores nas diferentes etapas de projeto com restrição volumétrica de 15\%.

\begin{tabular}{lccccc}
\hline $\begin{array}{l}\text { Grau de } \\
\text { liberdade }\end{array}$ & $\begin{array}{c}\text { Restrição } \\
\text { imposta ao } \\
\text { deslocamento }\end{array}$ & $\begin{array}{c}\text { Deslocamento no } \\
\text { final do processo } \\
\text { de OT }\end{array}$ & $\begin{array}{c}\text { Deslocamento } \\
\text { obtido na análise } \\
\text { do modelo de EF }\end{array}$ & $\begin{array}{c}\text { Energia de } \\
\text { deformação no } \\
\text { final da OT }\end{array}$ & $\begin{array}{c}\text { Energia de } \\
\text { deformação na } \\
\text { análise de EF }\end{array}$ \\
\hline A horizontal & $\begin{array}{c}-2,00 \mathrm{~mm} \\
(\text { valor mínimo })\end{array}$ & $-2,00 \mathrm{~mm}$ & $-2,75 \mathrm{~mm}$ & & $1250 \mathrm{Nmm}$ \\
B vertical & $\begin{array}{c}+1,00 \mathrm{~mm} \\
(\text { valor mínimo })\end{array}$ & $+1,00 \mathrm{~mm}$ & $+1,30 \mathrm{~mm}$ & & $1325 \mathrm{Nmm}$ \\
\hline
\end{tabular}

Tabela 4.5 - Comparação entre os valores nas diferentes etapas de projeto com restrição volumétrica de $20 \%$.

\begin{tabular}{lccccc}
\hline $\begin{array}{l}\text { Grau de } \\
\text { liberdade }\end{array}$ & $\begin{array}{c}\text { Restrição } \\
\text { imposta ao } \\
\text { deslocamento }\end{array}$ & $\begin{array}{c}\text { Deslocamento no } \\
\text { final do processo } \\
\text { de OT }\end{array}$ & $\begin{array}{c}\text { Deslocamento } \\
\text { obtido na análise } \\
\text { do modelo de EF }\end{array}$ & $\begin{array}{c}\text { Energia de } \\
\text { deformação no } \\
\text { final da OT }\end{array}$ & $\begin{array}{c}\text { Energia de } \\
\text { deformação na } \\
\text { análise de EF }\end{array}$ \\
\hline A horizontal & $\begin{array}{c}-2,00 \mathrm{~mm} \\
(\text { valor mínimo })\end{array}$ & $-2,00 \mathrm{~mm}$ & $-2,86 \mathrm{~mm}$ & $1250 \mathrm{Nmm}$ & $1297 \mathrm{Nmm}$ \\
B vertical & $\begin{array}{c}+1,00 \mathrm{~mm} \\
(\text { valor mínimo })\end{array}$ & $+1,00 \mathrm{~mm}$ & $+1,35 \mathrm{~mm}$ & & \\
\hline
\end{tabular}

Tabela 4.6 - Comparação entre os valores nas diferentes etapas de projeto com restrição volumétrica de $25 \%$.

\begin{tabular}{|c|c|c|c|c|c|}
\hline $\begin{array}{l}\text { Grau de } \\
\text { liberdade }\end{array}$ & $\begin{array}{c}\text { Restrição } \\
\text { imposta ao } \\
\text { deslocamento }\end{array}$ & $\begin{array}{c}\text { Deslocamento no } \\
\text { final do processo } \\
\text { de OT }\end{array}$ & $\begin{array}{c}\text { Deslocamento } \\
\text { obtido na análise } \\
\text { do modelo de EF }\end{array}$ & $\begin{array}{c}\text { Energia de } \\
\text { deformação no } \\
\text { final da OT } \\
\end{array}$ & $\begin{array}{c}\text { Energia de } \\
\text { deformação na } \\
\text { análise de EF }\end{array}$ \\
\hline A horizontal & $\begin{array}{c}-2,00 \mathrm{~mm} \\
\text { (valor mínimo) }\end{array}$ & $-2,00 \mathrm{~mm}$ & $-2,93 \mathrm{~mm}$ & & \\
\hline B vertical & $\begin{array}{c}+1,00 \mathrm{~mm} \\
\text { (valor mínimo) }\end{array}$ & $+1,00 \mathrm{~mm}$ & $+1,37 \mathrm{~mm}$ & $1250 \mathrm{Nmm}$ & 1317 Nmm \\
\hline
\end{tabular}




\subsection{AVALIAÇÃO DO EFEITO DA VARIAÇÃO DA RIGIDEZ E DO DESLOCAMENTO DA MOLA}

\subsubsection{Resultado inicial para referência}

Nesta seção, verifica-se o efeito provocado pela variação de dois parâmetros importantes do processo de otimização, sendo estes: a rigidez da mola que será deformada pelo mecanismo e a restrição imposta ao deslocamento relacionado a tal deformação. O problema de projeto de mecanismo flexível representado pela Figura 4.60 será utilizado como referência para as seções 4.6.2, 4.6.3 e 4.6.4.

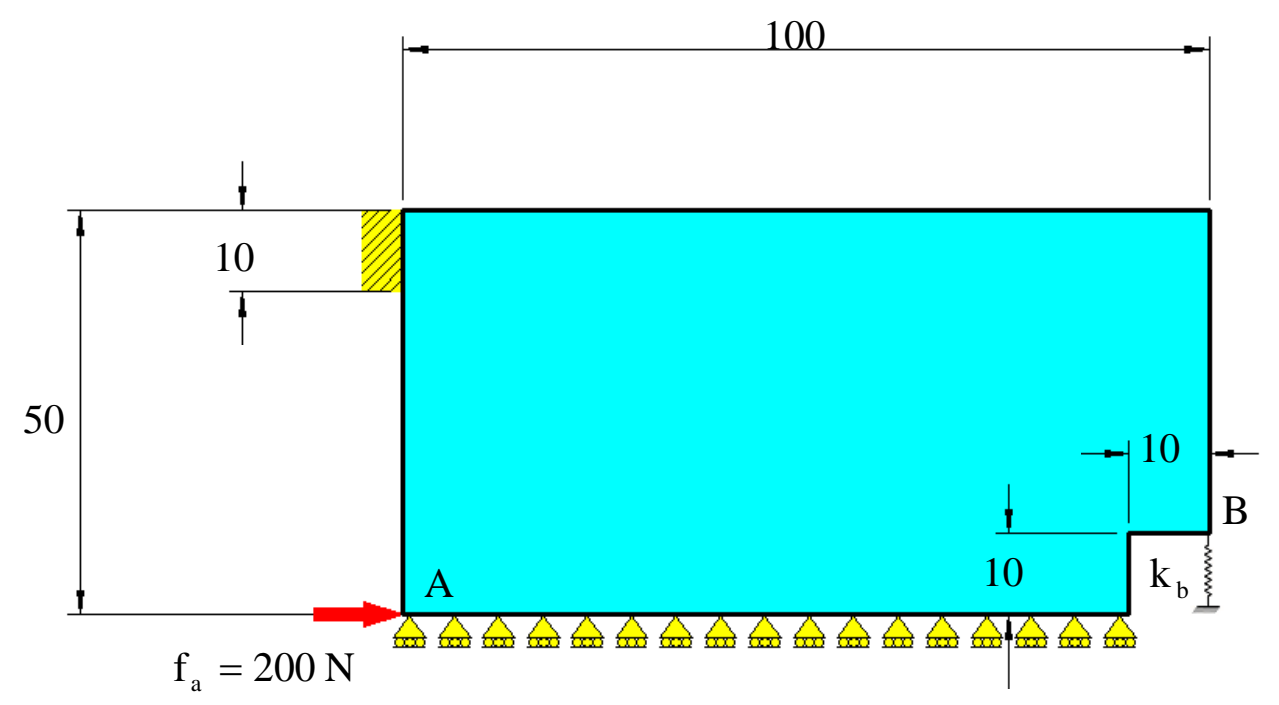

Figura 4.60 - Representação do problema de otimização.

Considerando que o material base a ser distribuído no domínio fixo mostrado na Figura 4.60 será o nylon, a força representada por $\mathrm{f}_{\mathrm{a}}$, de intensidade igual a $200 \mathrm{~N}$, deverá provocar um deslocamento máximo de $2 \mathrm{~mm}$ no seu ponto de aplicação, bem como um deslocamento mínimo de $1 \mathrm{~mm}$ na compressão da mola de rigidez $\mathrm{k}_{\mathrm{b}}$, cujo valor é igual a 50 N/mm. Restringiu-se o material do mecanismo em 30\% do volume do domínio fixo, o qual foi discretizado em 3617 elementos finitos. Adotou-se o valor de 0,001 como estimativa inicial atribuída a todas as variáveis de projeto. O raio de abrangência considerado para o 
esquema de filtragem corresponde a 1,35 mm. As restrições de deslocamento apresentaram-se ativas ao final do processo de otimização. Nestas condições, a topologia encontrada como solução pode ser vista na Figura 4.61, cujas áreas destacadas servirão de referência para a comparação com outros resultados.

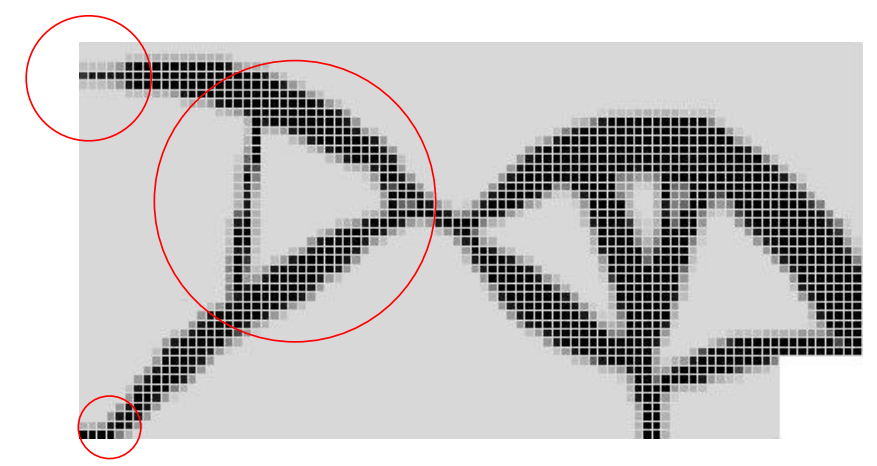

Figura 4.61 - Topologia para compressão mínima da mola equivalente a $1 \mathrm{~mm} \mathrm{e}_{\mathrm{b}}=50 \mathrm{~N} / \mathrm{mm}$.

\subsubsection{Redução da restrição imposta ao deslocamento de saída}

Nesta seção, o valor de $0,5 \mathrm{~mm}$ foi atribuído à restrição de deslocamento correspondente à deformação da mola. Desta forma, o problema de projeto proposto na seção anterior foi novamente solucionado. A Figura 4.62 demonstra o resultado encontrado.

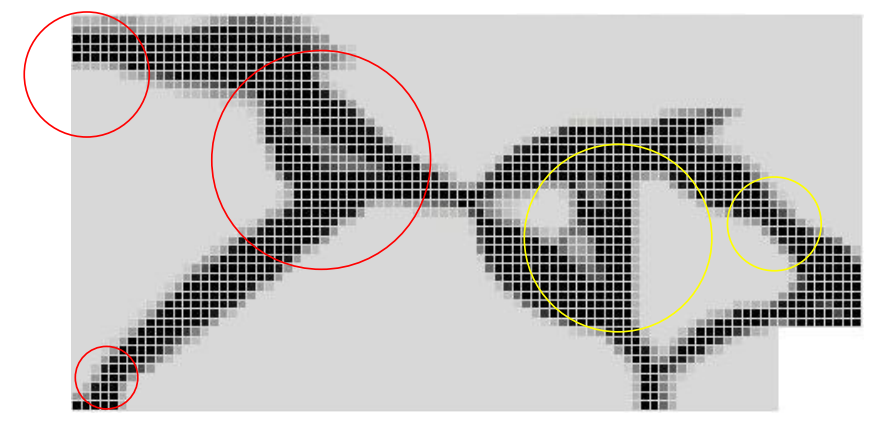

Figura 4.62 - Topologia para compressão mínima da mola equivalente a $0,5 \mathrm{~mm} \mathrm{e}_{\mathrm{b}}=50 \mathrm{~N} / \mathrm{mm}$.

A topologia da Figura 4.62 apresenta diferenças visíveis se comparada àquela da Figura 4.61. Contudo, um dado importante diz respeito ao deslocamento do ponto de entrada, cujo valor correspondente a 1,20 mm indica que a restrição associada ao ponto não foi ativada no final do processo de otimização. Através desta observação, conclui-se que a redução da 
energia necessária à deformação da mola determinou uma topologia mais rígida, através da qual menos energia de deformação é fornecida e absorvida. Este aspecto mais rígido pode ser observado por meio da comparação entre as regiões destacadas pelas circunferências vermelhas das Figuras 4.61 e 4.62. O resultado obtido nesta seção demonstra um comportamento bastante freqüente nos problemas solucionados pela formulação (2.57). Nestes problemas, quando um menor valor é prescrito à deformação da mola, as regiões rígidas comumente geradas nas proximidades do ponto de entrada reduzem a quantidade de energia recebida pelo mecanismo, e, concomitantemente, permitem que esta seja transferida de forma mais eficiente aos pontos menos rígidos da topologia e à mola.

\subsubsection{Redução da rigidez da mola}

O problema de projeto proposto na seção 4.6.2 foi solucionado com base na consideração de um novo valor atribuído à rigidez da mola $\left(\mathrm{k}_{\mathrm{b}}=22,5 \mathrm{~N} / \mathrm{mm}\right)$. A solução obtida esta demonstrada na próxima figura.

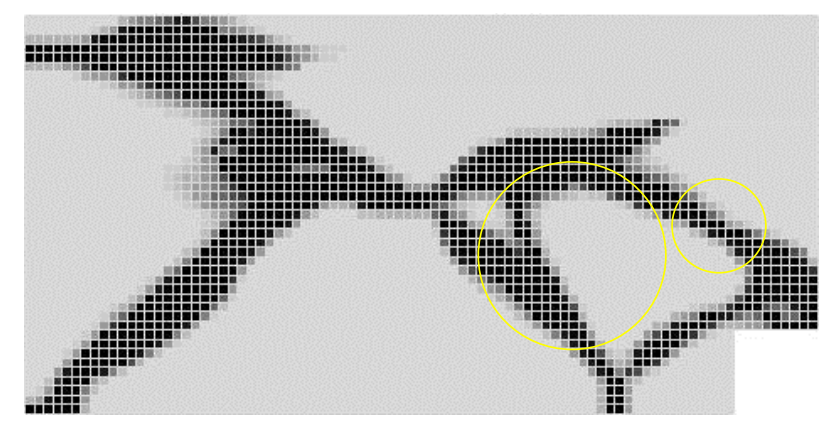

Figura 4.63 - Topologia para compressão mínima da mola equivalente a 0,5 mm e $\mathrm{k}_{\mathrm{b}}=22,5 \mathrm{~N} / \mathrm{mm}$.

Com exceção das regiões delimitadas pelas circunferências amarelas, a topologia da Figura 4.63 tem um aspecto similar àquela da Figura 4.62. Adicionalmente, observou-se que o deslocamento do ponto de entrada corresponde a 1,25 mm, logo, em ambos os casos, a força na entrada realiza praticamente o mesmo trabalho, apesar da topologia demonstrada pela Figura 4.63 estar vinculada a uma mola menos rígida. Com base nestes dados, conclui-se facilmente que ocorreu uma absorção maior de energia de deformação por parte da solução apontada nesta seção. Este fato pode ser explicado através da comparação entre as regiões 
destacadas pelas circunferências amarelas das Figuras 4.62 e 4.63. As regiões em destaque na Figura 4.63 têm um aspecto que sugere menos rigidez, e, portanto, maior capacidade de absorver a energia fornecida. A exemplo do que foi mostrado na seção anterior, este comportamento também é bastante comum para os problemas que empregam a formulação (2.57) como meio de solução, pois a redução de rigidez imposta à mola pode ocorrer também nas regiões do mecanismo que são mais próximas a ela, transferindo a energia - que inicialmente seria armazenada na própria mola - para o interior do mecanismo flexível.

\subsubsection{Supressão da mola e da restrição de saída}

Até agora, a formulação (2.57) foi utilizada para projetar mecanismos flexíveis menos suscetíveis às “dobradiças”. No entanto, cabe aqui uma investigação de interesse mais amplo. Com o objetivo de responder aos questionamentos que surgem sobre os possíveis resultados obtidos com a supressão da mola e da restrição de saída, o problema proposto na seção 4.6.1 foi solucionado de acordo com estas condições. A topologia relacionada pode ser vista a seguir.

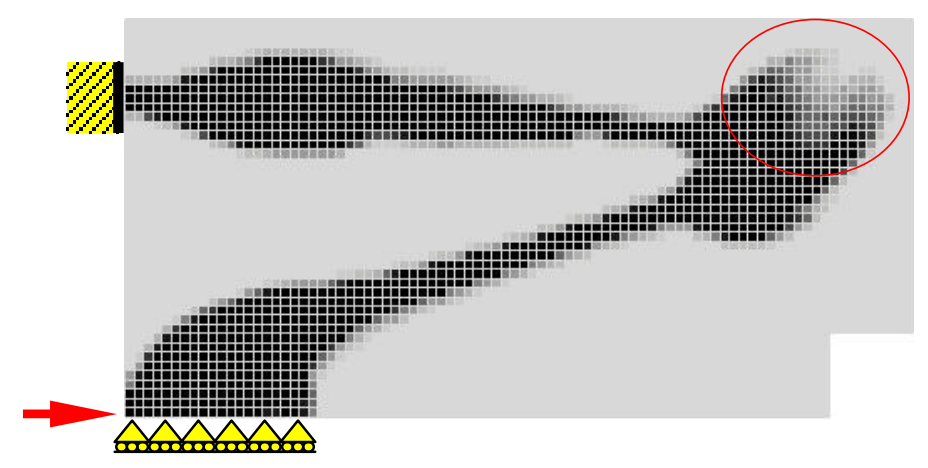

Figura 4.64 - Topologia obtida para o problema sem mola e sem ponto de saída.

Com a desconsideração da mola e de qualquer outra restrição de deslocamento que não esteja associada a um ponto de entrada, o problema de otimização deixa de ser aplicável ao projeto de mecanismos flexíveis, sendo, no entanto, mais apropriado ao projeto de estruturas rígidas, para as quais a flexibilidade média máxima pode ser indiretamente prescrita através das restrições de deslocamento impostas aos pontos de aplicação das forças ativas. Para o caso da topologia demonstrada pela Figura 4.64, a única restrição de deslocamento 
considerada ficou ativa no final do processo de otimização. A topologia assumiu um aspecto completamente diferente do que os observados nas seções anteriores. Vê-se, inclusive, que a estrutura não está conectada ao “antigo” ponto de saída definido para os mecanismos flexíveis. A região destacada pela Figura 4.64 demonstra uma escala de cinza mais extensa do que a existente no contorno da topologia, logo, os gradientes das variáveis de projeto da referida região são menores. Assim, à luz da discussão feita na seção 4.3.4, na qual o filtro espacial de densidades é classificado como um filtro "passa-baixa”, pode-se supor que as maiores escalas de cinza demonstradas na região destacada não são provocadas pela ação do filtro, cuja atuação é mais efetiva no contorno da estrutura, onde os gradientes são maiores, certamente mais próximos de uma restrição implícita.

\subsection{PROJETO DE ESTRUTURAS RÍGIDAS}

\subsubsection{Comentários iniciais}

A seção 4.6.4 serve como motivação para discutir os resultados obtidos com o código de otimização - originalmente elaborado para projetar mecanismos flexíveis - quando aplicado ao projeto de estruturas rígidas. Não há, neste texto, a pretensão de considerar este código como uma ferramenta pronta e completamente ajustada a esta tarefa. O objetivo prioritário deste trabalho é abordar as questões que envolvem o projeto de mecanismos flexíveis isentos, o quanto possível, de “dobradiças”. Contudo, alguns dados colhidos a partir desta aplicação alternativa do código podem ser úteis ao melhor entendimento da formulação, bem como aos futuros trabalhos relacionados ao projeto de estruturas rígidas, que, no MOT, vêm sendo obtidas por meio da minimização da flexibilidade média segundo uma dada quantidade de material disponível (SIGMUND, 2001a). A utilização do código de otimização com o propósito de obter topologias rígidas requer a atribuição do valor nulo tanto à rigidez da mola quanto ao deslocamento mínimo daquele que seria o ponto de saída de um eventual mecanismo flexível. O grau de liberdade global deste falso ponto de saída deverá ser o mesmo do ponto de entrada. Na prática, o efeito corresponde à consideração de duas restrições para o deslocamento do único ponto em discussão, que, ao final do processo de otimização, apresentará um deslocamento compreendido entre zero e um valor máximo prescrito através 
da restrição de entrada. Este valor máximo determina, indiretamente, a máxima flexibilidade média da estrutura. Em princípio, o projeto de estruturas rígidas está relacionado apenas ao ajuste das restrições de deslocamento. No entanto, os resultados apresentam alguns aspectos que demandam uma investigação mais cuidadosa. Embora esta investigação não tenha sido feita com a devida profundidade neste trabalho, os referidos aspectos são demonstrados nas próximas seções.

\subsubsection{Variação do coeficiente de penalização das densidades}

Ao contrário do projeto de mecanismos flexíveis, projetar estruturas rígidas a partir da formulação (2.57) é um tanto mais difícil sob a questão do controle das escalas de cinza da topologia. Para ilustrar esta questão, bem como outras com respeito a este capítulo, adotou-se o problema de otimização da Figura 4.65.

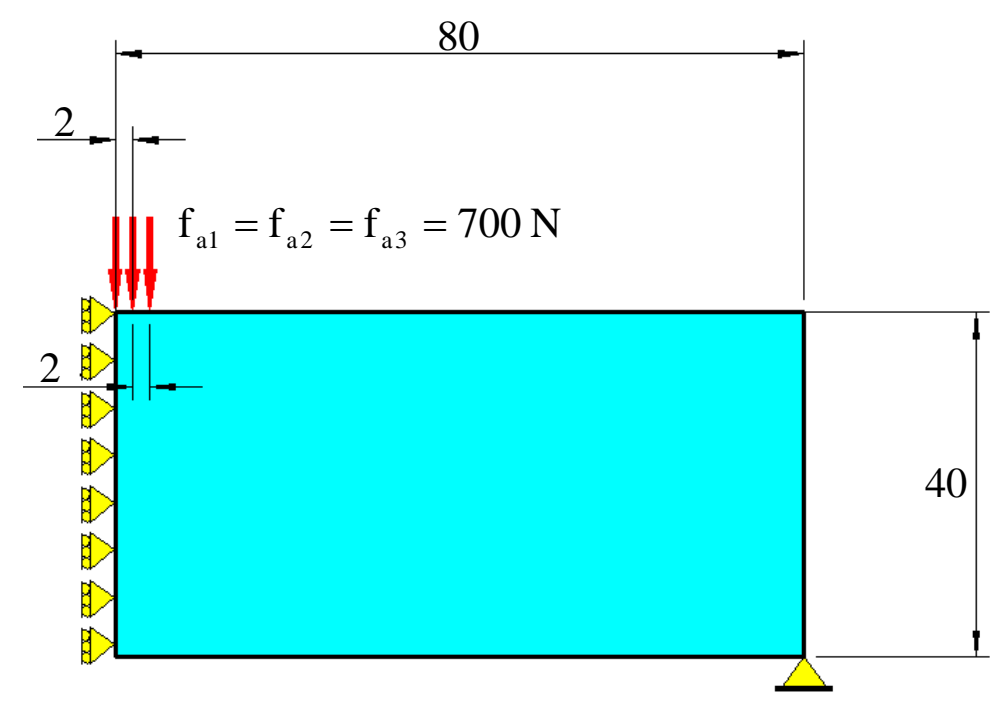

Figura 4.65 - Representação do problema de otimização.

A estrutura projetada, constituída de aço, deverá corresponder a 30\% do volume do domínio fixo, cuja geometria foi definida de acordo com a simetria do problema. A fim de aplicar o processo de otimização, discretizou-se o domínio em 3200 elementos finitos. O problema proposto foi primeiramente solucionado através do código elaborado por Sigmund (2001a) - A 99 line topology optimization code written in Matlab - no qual está implementada 
a formulação tradicional para o projeto de estruturas - minimização da flexibilidade média segundo uma dada restrição de volume. A topologia obtida como solução é demonstrada na próxima figura, bem como o mapa de tensões relacionado à análise estática efetuada sobre estrutura final projetada.

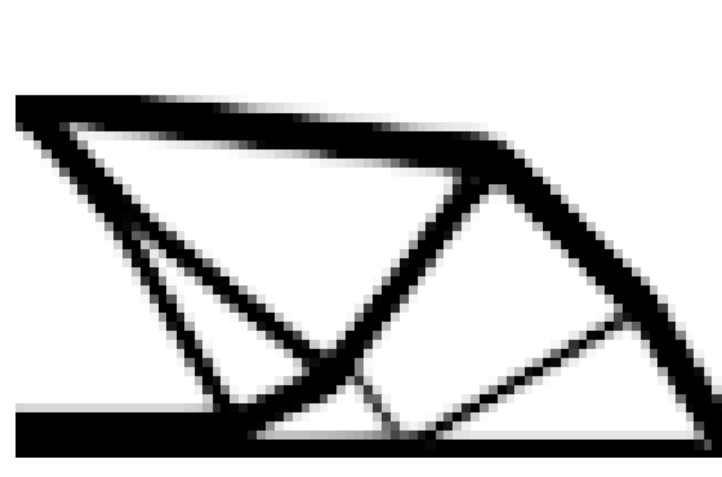

(a)

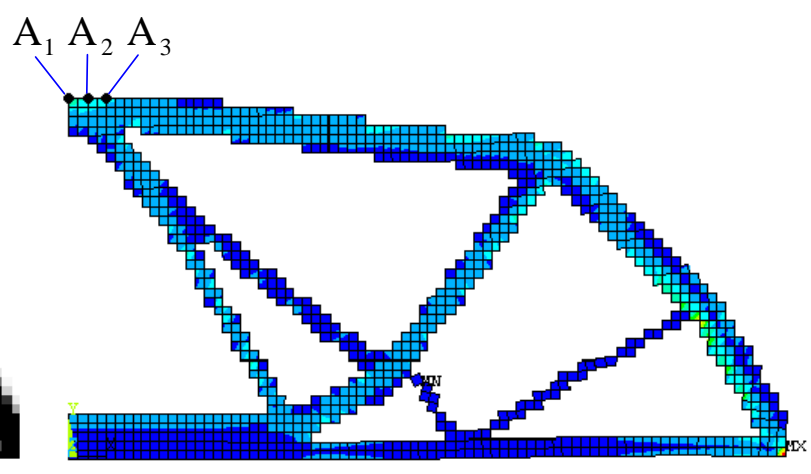

(b)

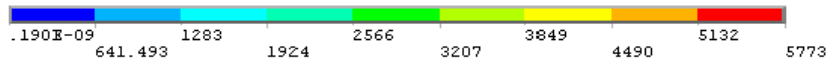

Figura 4.66 - (a) solução do problema de otimização topológica; (b) mapa de cores indicando as tensões segundo o critério de resistência de von Mises $\left(\mathrm{em} \mathrm{N} / \mathrm{mm}^{2}\right)$.

O código proposto por Sigmund apresenta duas diferenças principais em relação à rotina elaborada neste trabalho. Inicialmente, ele adota um critério de optimalidade como método de solução do problema de otimização, e, por fim, ele considera um filtro espacial de sensibilidades (SIGMUND, 1997) para garantir a existência da solução. Este tipo de filtro também utiliza um raio de abrangência para determinar os elementos que alteram o gradiente da função objetivo inicialmente calculado em relação à densidade do elemento sobre o qual o esquema de filtragem está sendo considerado. Desta forma, obteve-se a topologia da Figura 4.66a com um raio de abrangência igual a 1,15 mm. Já os pontos $A_{1}, A_{2}$ e $A_{3}$ apresentaram os deslocamentos verticais - observados no sentido de atuação das forças - iguais a 1,26 mm, 1,25 mm e 1,23 mm, respectivamente. Na aplicação da formulação (2.57) à solução do problema representado pela Figura 4.65, considera-se que cada uma das três forças deverá provocar um deslocamento máximo de 1,26 mm. Assim, deve-se obter uma estrutura com a mesma flexibilidade média do que a observada para a topologia da Figura 4.66a. Nestas condições, a partir de uma estimativa inicial de otimização igual a 0,15 - atribuída a todas as variáveis de projeto - e tendo sido adotado o raio de abrangência correspondente a 1,25 mm para o esquema de filtragem das densidades, foram obtidas as duas topologias demonstradas na figura seguinte. 

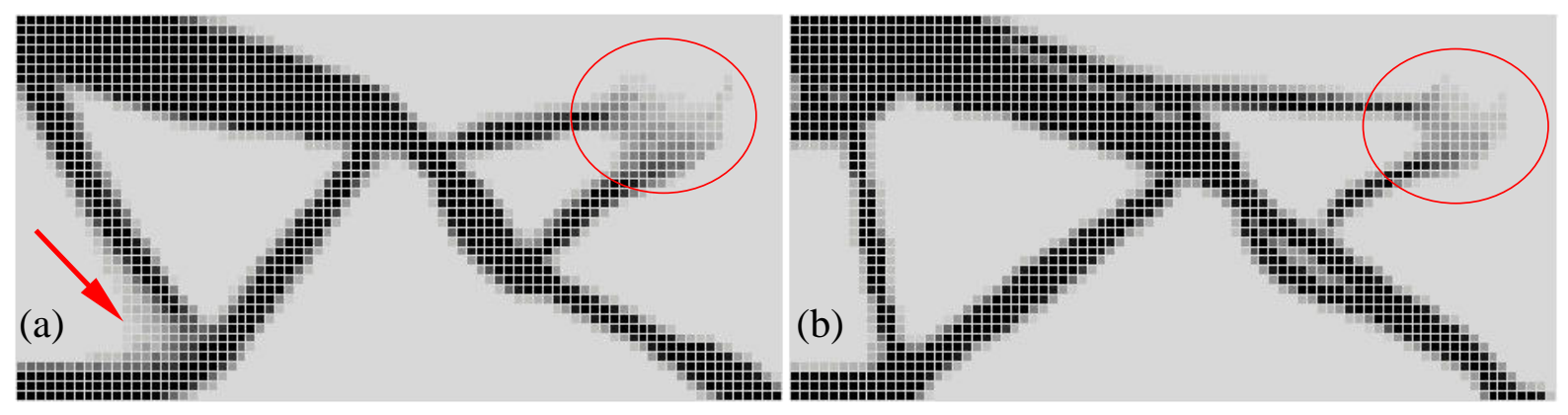

Figura 4.67 - (a) topologia obtida para $n=2$; (b) topologia obtida para $n=4$.

Diferentemente do projeto de mecanismos flexíveis, a aplicação da formulação (2.57) ao projeto de estruturas rígidas produz soluções distintas para coeficientes de penalização distintos, conforme se vê na Figura 4.67. Com respeito às escalas de cinza presentes nas regiões realçadas pelas elipses, verifica-se que sofrem pouca influência em relação à variação do coeficiente de penalização. Observa-se que estas regiões têm um aspecto ligeiramente similar àquela destacada pela Figura 4.64 na seção 4.6.4. Estes resultados ilustram a pouca sensibilidade que as escalas de cinza presentes em áreas específicas da topologia têm em relação à variação dos dois principais parâmetros capazes de controlá-las - o raio de abrangência do filtro espacial de densidades e o coeficiente de penalização das densidades.

\subsubsection{Variação da estimativa inicial de otimização}

Ainda com respeito à figura anterior, nota-se que o aumento do coeficiente de penalização reduziu as escalas de cinza presentes inicialmente na área apontada pela seta indicativa da Figura 4.67a. No intuito de evitar, o quanto possível, as regiões acinzentadas mais extensas, os próximos resultados relacionados ao problema da Figura 4.65 consideram o raio de abrangência do esquema de filtragem igual a $1,15 \mathrm{~mm}$ e o coeficiente de penalização das densidades igual a 4 (quatro). Assim, a seguir, demonstram-se as topologias obtidas a partir das estimativas iniciais de otimização correspondentes a 0,15, 0,50 e 0,85, respectivamente - cada uma delas atribuídas a todas as variáveis de projeto. 


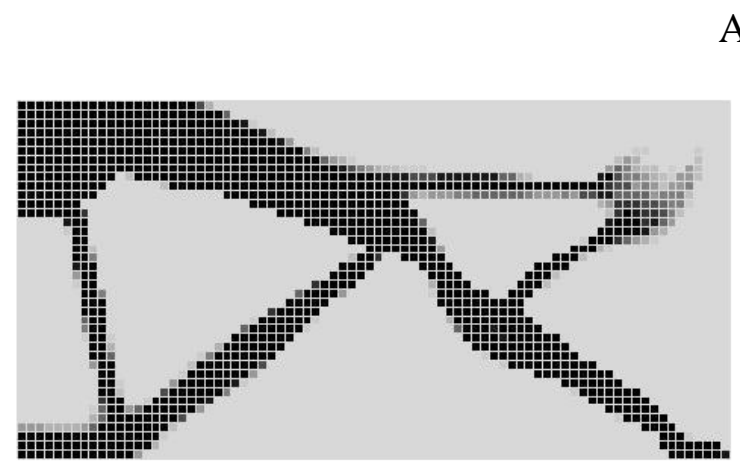

(a)

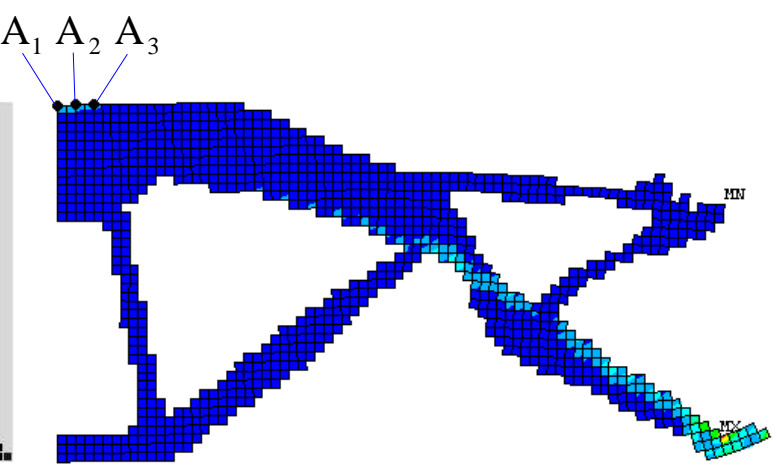

(b)

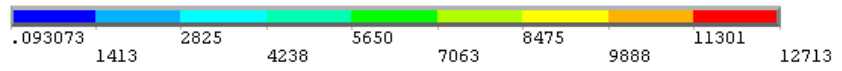

Figura 4.68 - (a) solução do problema de otimização topológica com estimativa inicial de 0,15; (b) mapa de cores indicando as tensões segundo o critério de resistência de von Mises (em N/mm²).

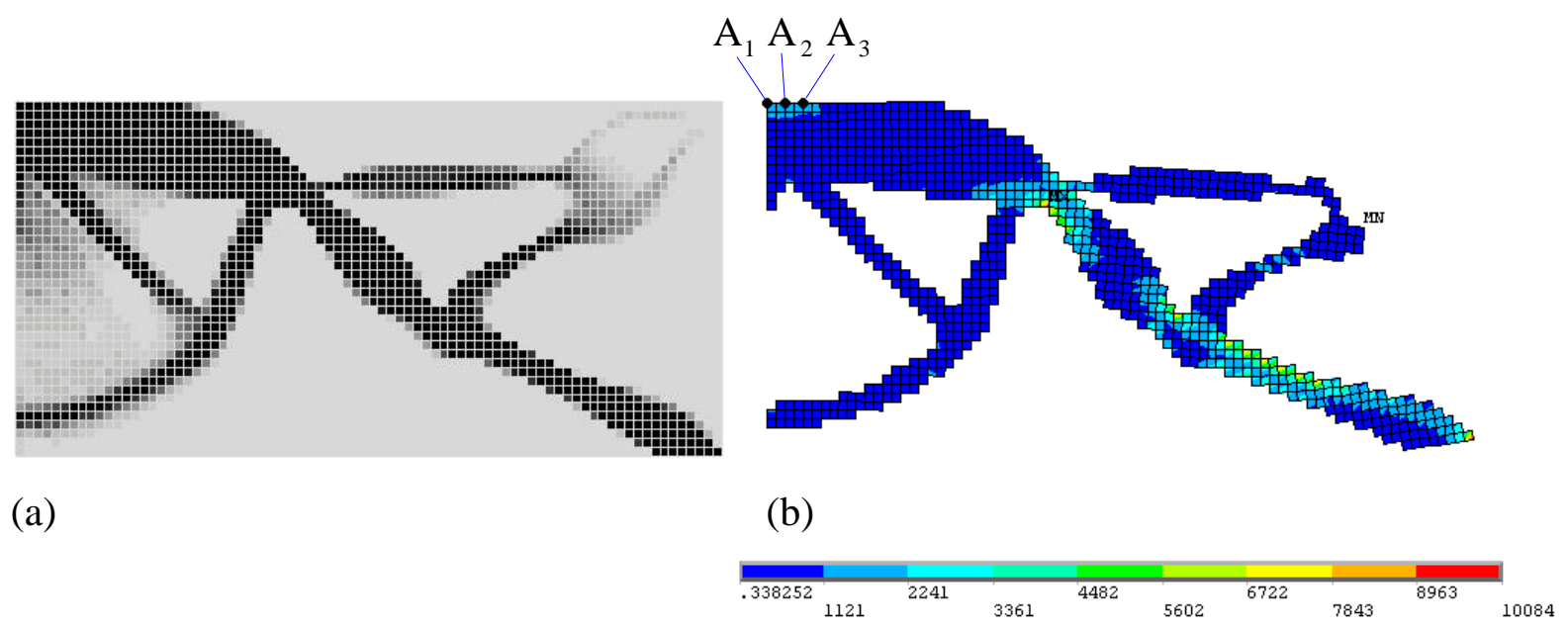

Figura 4.69 - (a) solução do problema de otimização topológica com estimativa inicial de 0,50; (b) mapa de cores indicando as tensões segundo o critério de resistência de von Mises (em N/mm²).

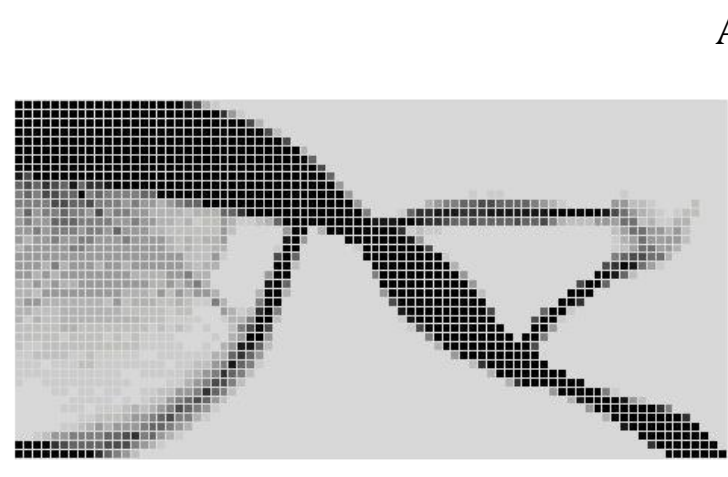

(a)

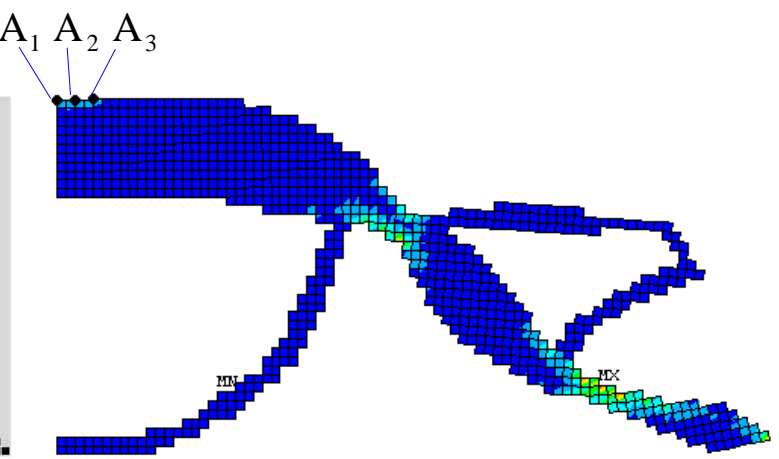

(b)

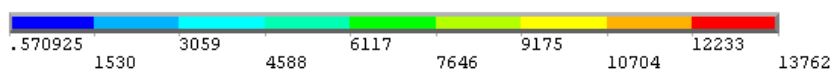

Figura 4.70 - (a) solução do problema de otimização topológica com estimativa inicial de 0,85; (b) mapa de cores indicando as tensões segundo o critério de resistência de von Mises (em N/mm²). 
Assim como no projeto de mecanismos flexíveis, as topologias das Figuras 4.68, 4.69 e 4.70 indicam que a formulação abordada neste trabalho, quando aplicada ao projeto de estruturas rígidas, também produz múltiplos “ótimos” locais. Nas Figuras 4.68b, 4.69b e 4.70b, as análises estáticas dos modelos, que foram elaborados, respectivamente, a partir da interpretação das topologias apresentadas pelas Figuras 4.68a, 4.69a e 4.70a, apontam tensões mecânicas maiores e mais concentradas do que as tensões indicadas pela Figura 4.66b. Estes resultados sugerem que as restrições de deslocamento impostas aos pontos de aplicação das cargas podem provocar o enrijecimento da região que contém estes pontos. Com isso, surgem áreas da topologia nas quais a flexibilidade e, conseqüentemente, as tensões mecânicas se concentram. Em relação à extensão das escalas de cinza, constatou-se o seu aumento na medida em que o valor inicial das variáveis de projeto foi incrementado. Este comportamento - particularmente observado no problema de otimização proposto para esta seção - dificultou as interpretações dos resultados e, portanto, prejudicou o desempenho das estruturas representadas pelos modelos de EF. Esta queda no desempenho pode ser notada nas Tabelas 4.8, 4.9 e 4.10, que correspondem às Figuras 4.68, 4.69 e 4.70, respectivamente. A Tabela 4.7, por sua vez, refere-se a Figura 4.66, na qual a solução apresentada foi obtida através do código de otimização elaborado por Sigmund (2001a). Nestes quatro casos, as restrições volumétricas mantiveram-se ativas no final de seus respectivos processos de otimização.

Tabela 4.7 - Deslocamentos nas duas etapas de projeto, sendo a OT feita através do código de Sigmund (2001a).

\begin{tabular}{lccc}
\hline Grau de liberdade & $\begin{array}{c}\text { Restrição imposta ao } \\
\text { deslocamento }\end{array}$ & $\begin{array}{c}\text { Deslocamento no final do } \\
\text { processo de OT }\end{array}$ & $\begin{array}{c}\text { Deslocamento obtido na } \\
\text { análise do modelo de EF }\end{array}$ \\
\hline A1 vertical & (não aplicável) & $-1,26 \mathrm{~mm}$ & $-1,32 \mathrm{~mm}$ \\
A2 vertical & (não aplicável) & $-1,25 \mathrm{~mm}$ & $-1,31 \mathrm{~mm}$ \\
A3 vertical & (não aplicável) & $-1,23 \mathrm{~mm}$ & $-1,29 \mathrm{~mm}$ \\
\hline
\end{tabular}

Tabela 4.8 - Deslocamentos nas duas etapas de projeto representadas pelas Figuras 4.68a e 4.68b.

\begin{tabular}{lccc}
\hline Grau de liberdade & $\begin{array}{c}\text { Restrição imposta ao } \\
\text { deslocamento }\end{array}$ & $\begin{array}{c}\text { Deslocamento no final do } \\
\text { processo de OT }\end{array}$ & $\begin{array}{c}\text { Deslocamento obtido na } \\
\text { análise do modelo de EF }\end{array}$ \\
\hline A1 vertical & $-1,26 \mathrm{~mm}$ (valor mínimo) & $-1,27 \mathrm{~mm}$ & $-1,77 \mathrm{~mm}$ \\
A2 vertical & $-1,26 \mathrm{~mm}$ (valor mínimo) & $-1,26 \mathrm{~mm}$ & $-1,76 \mathrm{~mm}$ \\
A vertical & $-1,26 \mathrm{~mm}$ (valor mínimo) & $-1,25 \mathrm{~mm}$ & $-1,75 \mathrm{~mm}$ \\
\hline
\end{tabular}

Tabela 4.9 - Deslocamentos nas duas etapas de projeto representadas pelas Figuras 4.69a e 4.69b.

\begin{tabular}{lccc}
\hline Grau de liberdade & $\begin{array}{c}\text { Restrição imposta ao } \\
\text { deslocamento }\end{array}$ & $\begin{array}{c}\text { Deslocamento no final do } \\
\text { processo de OT }\end{array}$ & $\begin{array}{c}\text { Deslocamento obtido na } \\
\text { análise do modelo de EF }\end{array}$ \\
\hline A 1 vertical & $-1,26 \mathrm{~mm}$ (valor mínimo) & $-1,26 \mathrm{~mm}$ & $-2,71 \mathrm{~mm}$ \\
A 2 vertical & $-1,26 \mathrm{~mm}$ (valor mínimo) & $-1,26 \mathrm{~mm}$ & $-2,70 \mathrm{~mm}$ \\
A 3 vertical & $-1,26 \mathrm{~mm}$ (valor mínimo) & $-1,25 \mathrm{~mm}$ & $-2,69 \mathrm{~mm}$ \\
\hline
\end{tabular}


Tabela 4.10 - Deslocamentos nas duas etapas de projeto representadas pelas Figuras 4.70a e 4.70b.

\begin{tabular}{lccc}
\hline Grau de liberdade & $\begin{array}{c}\text { Restrição imposta ao } \\
\text { deslocamento }\end{array}$ & $\begin{array}{c}\text { Deslocamento no final do } \\
\text { processo de OT }\end{array}$ & $\begin{array}{c}\text { Deslocamento obtido na } \\
\text { análise do modelo de EF }\end{array}$ \\
\hline A $_{1}$ vertical & $-1,26 \mathrm{~mm}$ (valor mínimo) & $-1,27 \mathrm{~mm}$ & $-2,97 \mathrm{~mm}$ \\
A $_{2}$ vertical & $-1,26 \mathrm{~mm}$ (valor mínimo) & $-1,26 \mathrm{~mm}$ & $-2,96 \mathrm{~mm}$ \\
A $_{3}$ vertical & $-1,26 \mathrm{~mm}$ (valor mínimo) & $-1,26 \mathrm{~mm}$ & $-2,95 \mathrm{~mm}$ \\
\hline
\end{tabular}

Dada a simetria do problema, apenas a sua metade foi considerada nos processos de otimização, assim, as configurações finais das estruturas completas estão demonstradas nas Figuras 4.71, 4.72, 4.73 e 4.74.

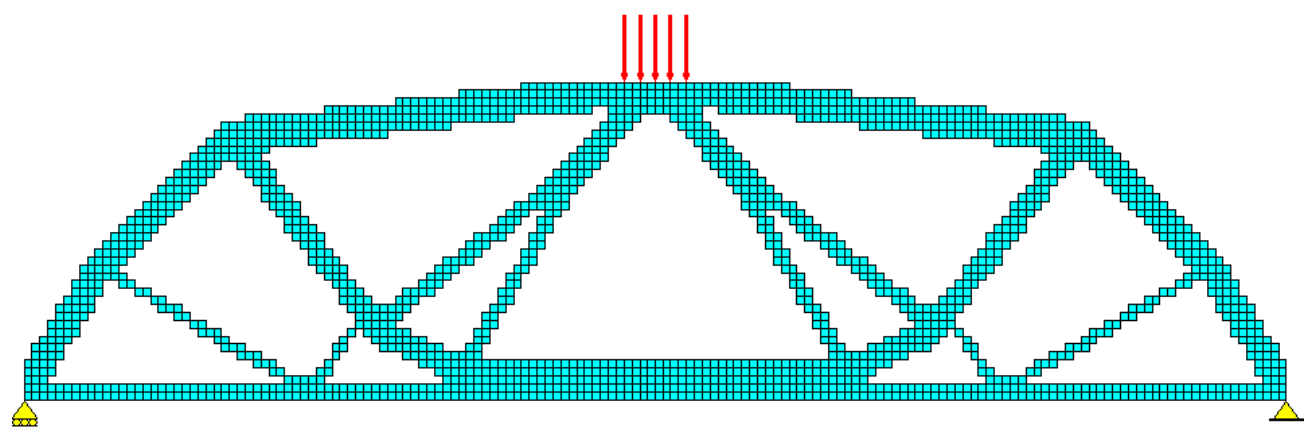

Figura 4.71 - Configuração final e completa da estrutura correspondente à Figura 4.66.

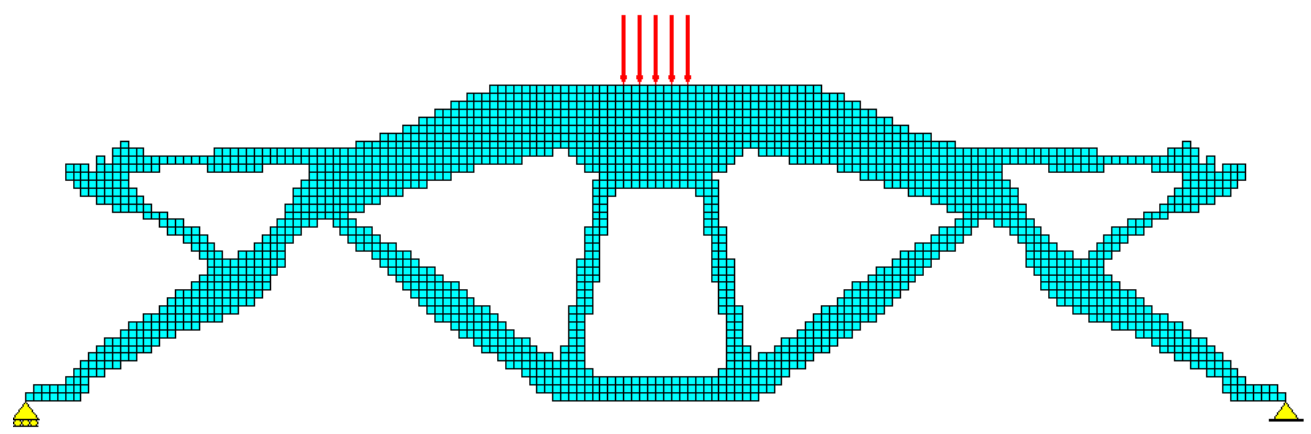

Figura 4.72 - Configuração final e completa da estrutura correspondente à Figura 4.68.

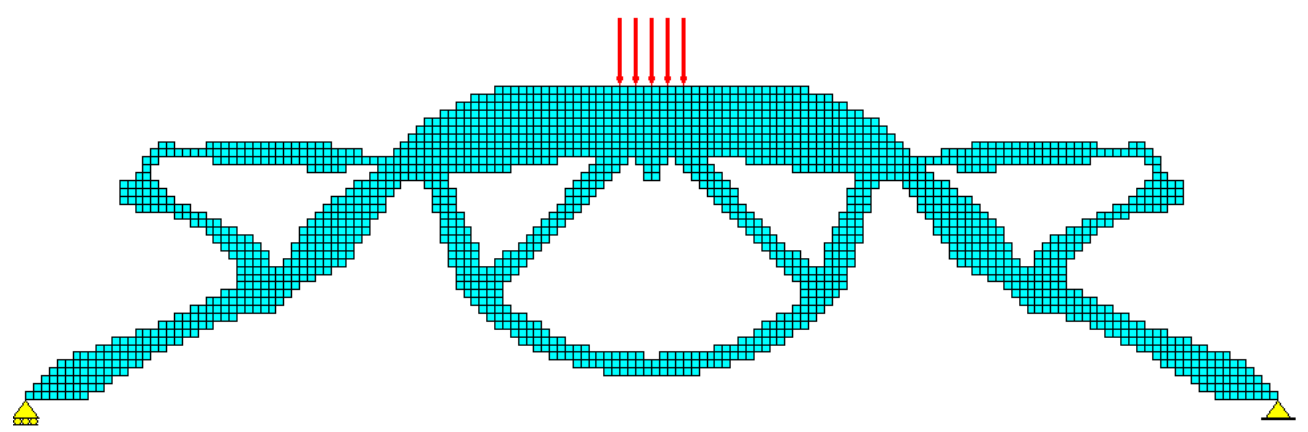

Figura 4.73 - Configuração final e completa da estrutura correspondente à Figura 4.69. 


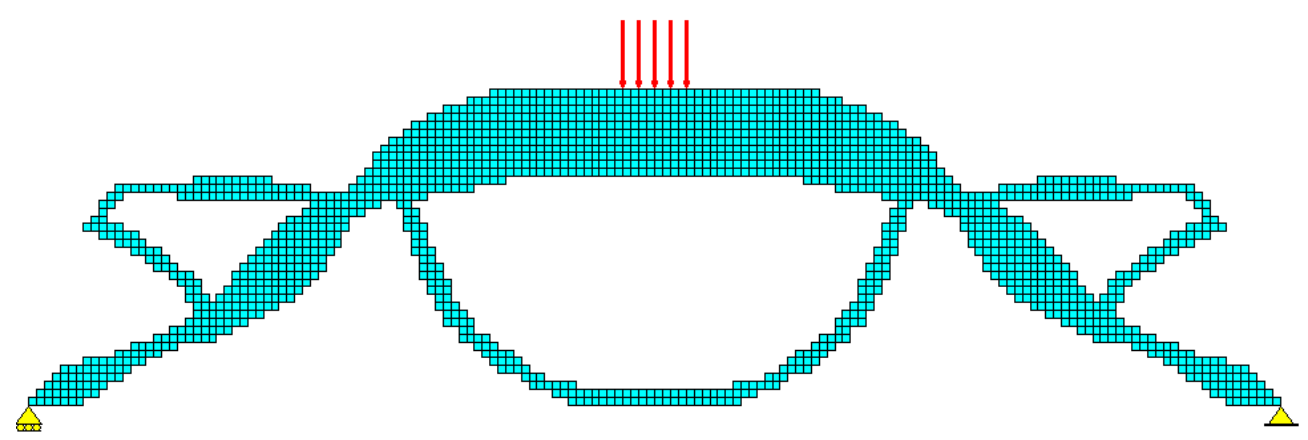

Figura 4.74 - Configuração final e completa da estrutura correspondente à Figura 4.70.

\subsection{MULTI-FLEXIBILIDADE}

\subsubsection{Comentários iniciais}

Nesta última seção do capítulo, apresentam-se os resultados relacionados à aplicação da maximização da energia de deformação ao projeto de mecanismos flexíveis submetidos a mais de um caso de carregamento (multi-flexibilidade). Para tanto, a formalização matemática do problema de otimização (2.60) - variante da formulação (2.57) - foi implementada no código computacional elaborado para este trabalho. Os dois problemas propostos na próxima seção consideram o projeto de mecanismos flexíveis submetidos a dois casos de carregamento. Contudo, conforme foi discutido na seção 2.6.5, a formulação (2.60) pode atender a um número indefinido de casos.

\subsubsection{Redução da ocorrência de “dobradiças” para problemas de multi-flexibilidade}

Assim como nos casos mais simples, nos quais apenas um carregamento é considerado, os problemas que envolvem a multi-flexibilidade, quando solucionados por meio da maximização da energia de deformação, também são menos suscetíveis a ocorrência de “dobradiças”. A Figura 4.75 representa um problema de projeto de mecanismo multi-flexível 
cujas soluções foram obtidas através de duas formulações distintas. Quando estas soluções são comparadas entre si, é possível verificar as diferenças acerca da questão das “dobradiças”.

(a)

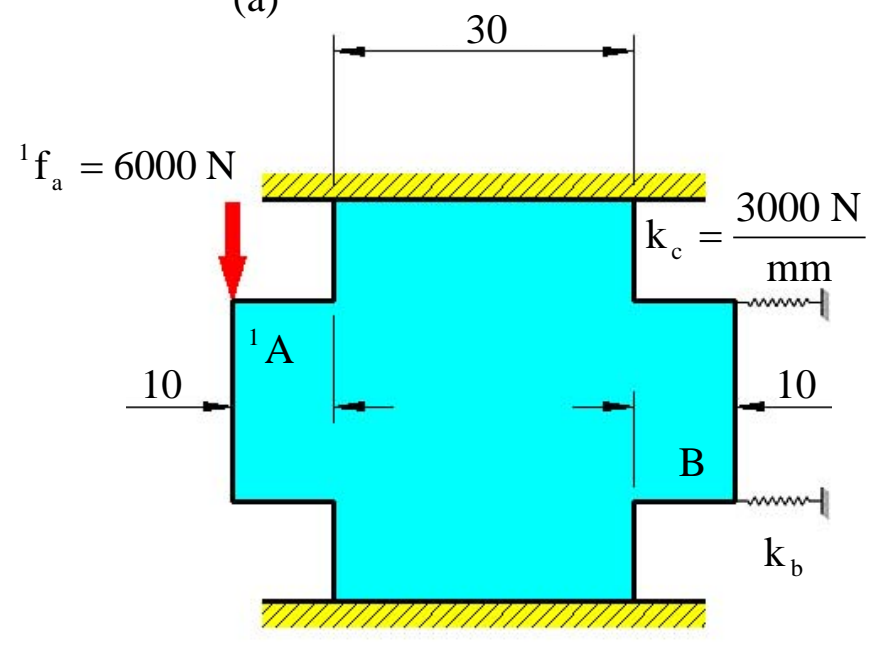

(b)

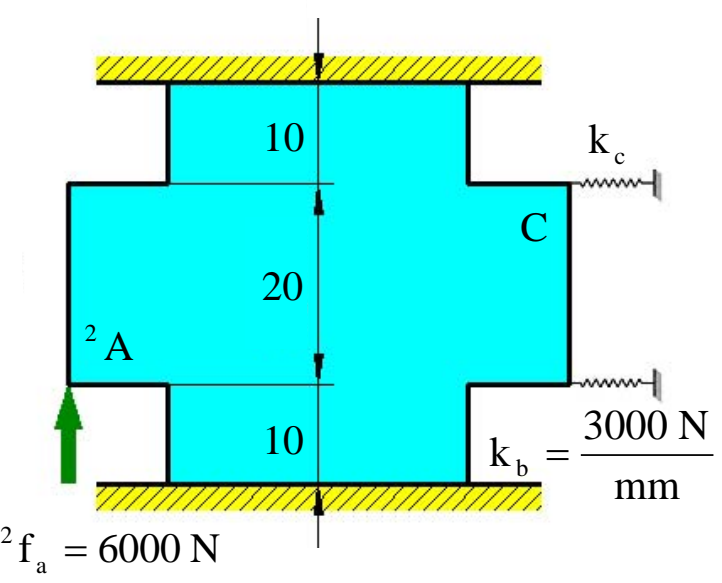

Figura 4.75 - (a) $1^{\circ}$ caso de carregamento do problema de OT; (b) $2^{\circ}$ caso de carregamento do problema de OT.

O aço será o material base a ser distribuído no domínio fixo mostrado pelas Figuras 4.75a e 4.75b. No primeiro caso de carregamento, a força representada por ${ }^{1} \mathrm{f}_{\mathrm{a}}$, cuja intensidade é igual a $6000 \mathrm{~N}$, deverá provocar um deslocamento máximo de $1 \mathrm{~mm}$ no seu ponto de aplicação, bem como um deslocamento mínimo de 0,5 mm na compressão da mola de rigidez $\mathrm{k}_{\mathrm{b}}$ igual a $3000 \mathrm{~N} / \mathrm{mm}$. No segundo caso de carregamento, a força representada por ${ }^{2} \mathrm{f}_{\mathrm{a}}$, para a qual a intensidade também assume valor igual a $6000 \mathrm{~N}$, provocará o mesmo deslocamento máximo de $1 \mathrm{~mm}$ no seu ponto de aplicação, bem como um deslocamento mínimo de 0,5 mm na tração da mola de rigidez $\mathrm{k}_{\mathrm{c}}$, cujo valor corresponde a $3000 \mathrm{~N} / \mathrm{mm}$. Restringiu-se o material do mecanismo em 30\% do volume do domínio fixo, o qual foi discretizado em 1600 elementos finitos. Adotou-se o valor de 0,15 como estimativa inicial atribuída a todas as variáveis de projeto. O raio de abrangência considerado para o esquema de filtragem corresponde a $1,10 \mathrm{~mm}$. O problema representado pela Figura 4.75 foi inicialmente proposto por Nishiwaki et al. (2001). Naquele trabalho, os autores utilizaram um código de otimização que emprega o método da homogeneização como modelo de material. Adotou-se, também, um esquema numérico de filtragem cuja aplicação incide sobre grupos de quatro elementos com um nó em comum - patch technique (BENDSØE, 1995), “varrendo”, 
desta forma, todo o domínio fixo de projeto. Além destas características mais gerais relacionadas ao MOT, o código de Nishiwaki et al. (2001) considera a maximização da energia mútua e minimização da flexibilidade média, ambas as otimizações feitas através de uma função multi-objetivo que compatibiliza todos os modos de deformação. Assim, foi possível obter a seguinte topologia para o problema proposto na Figura 4.75.

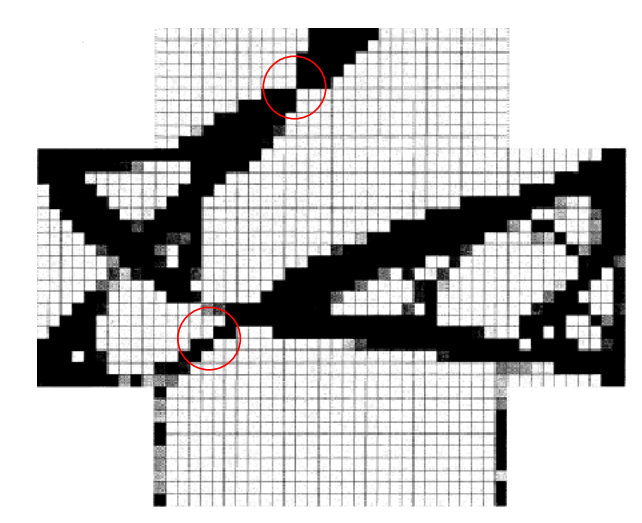

Figura 4.76 - Topologia extraída do artigo publicado por Nishiwaki et al. (2001).

A Figura 4.77, por sua vez, corresponde à solução obtida por meio da maximização da energia de deformação - formulação (2.60).

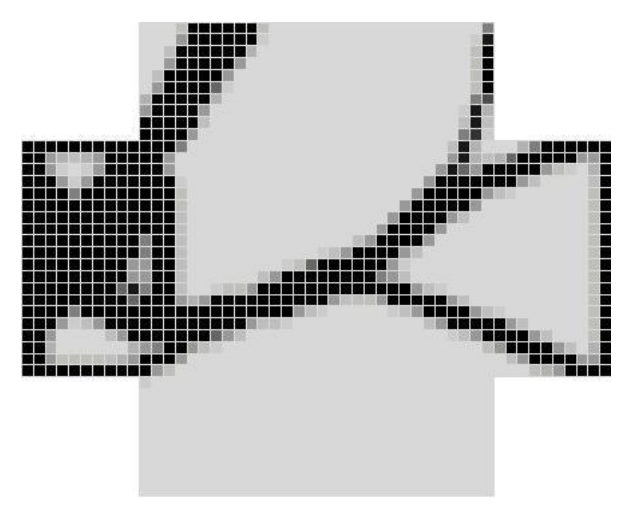

Figura 4.77 - Topologia obtida através da maximização da energia de deformação.

As regiões destacadas na topologia da Figura 4.76 demonstram as "dobradiças" resultantes do processo de otimização. Observa-se que este tipo de instabilidade não ocorre na Figura 4.77. Já as análises estáticas efetuadas sobre o modelo construído a partir da topologia da Figura 4.77 - análises realizadas em ambos os casos de carregamento - fornecem as configurações deformadas representadas pelas Figuras 4.78 e 4.79 . 


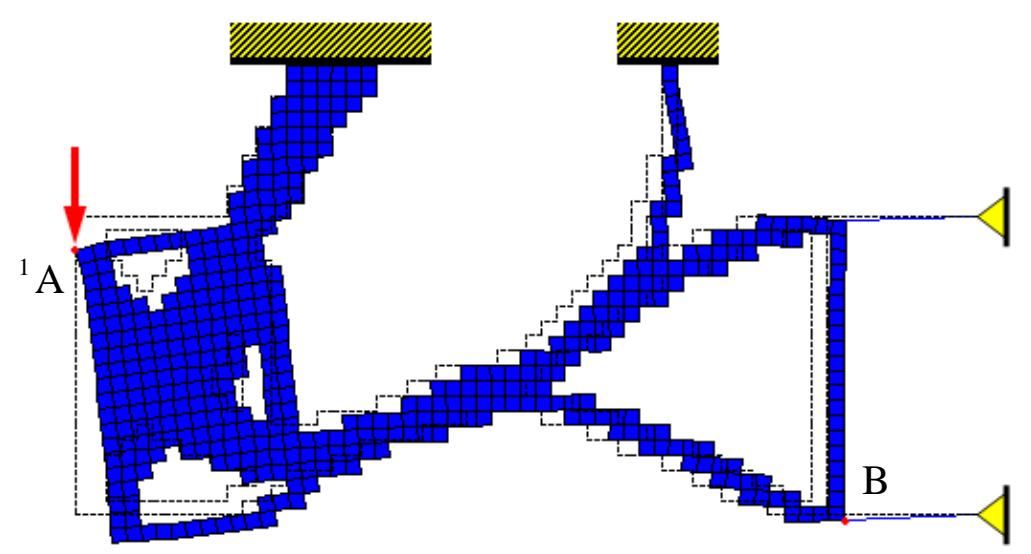

Figura 4.78 - Configuração deformada correspondente ao $1^{\circ}$ caso de carregamento.

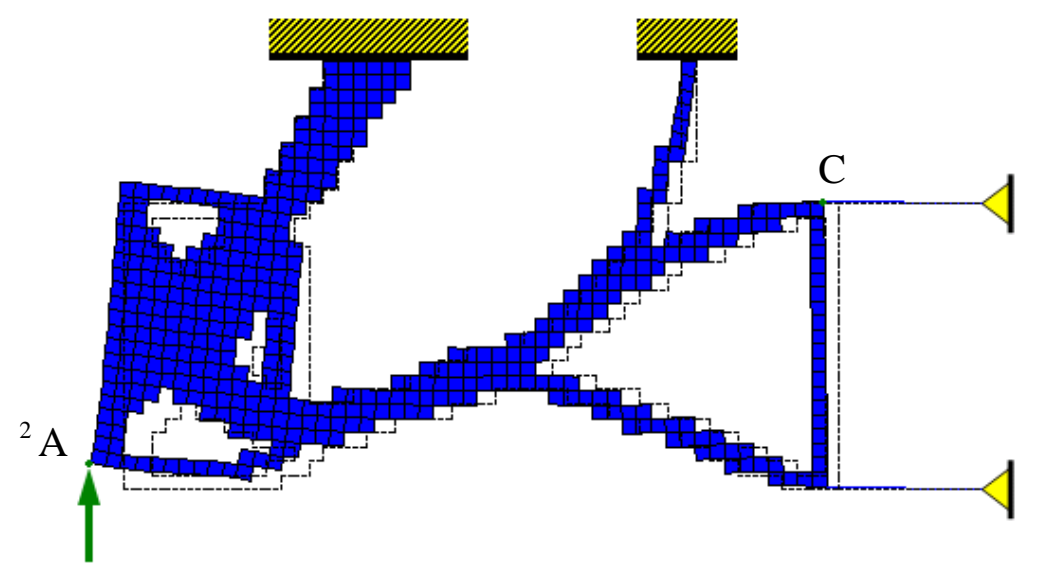

Figura 4.79 - Configuração deformada correspondente ao $2^{\circ}$ caso de carregamento.

A Tabela 4.11 traz os deslocamentos dos pontos ${ }^{1} \mathrm{~A},{ }^{2} \mathrm{~A}$, B e C observados em cada uma das etapas de projeto do mecanismo multi-flexível obtido através da maximização da energia de deformação.

Tabela 4.11 - Comparação entre os deslocamentos observados nas diferentes etapas de projeto.

\begin{tabular}{|c|c|c|c|c|}
\hline Carregamento & Grau de liberdade & $\begin{array}{c}\text { Restrição imposta ao } \\
\text { deslocamento }\end{array}$ & $\begin{array}{c}\text { Deslocamento no } \\
\text { final do processo de } \\
\text { OT }\end{array}$ & $\begin{array}{c}\text { Deslocamento obtido } \\
\text { na análise do modelo } \\
\text { de EF }\end{array}$ \\
\hline $1^{\circ}$ carregamento & ${ }^{1}$ A vertical & $\begin{array}{c}-1,00 \mathrm{~mm} \\
\text { (valor mínimo) }\end{array}$ & $-0,99 \mathrm{~mm}$ & $-1,17 \mathrm{~mm}$ \\
\hline $1^{\circ}$ carregamento & B horizontal & $\begin{array}{c}+0,50 \mathrm{~mm} \\
\text { (valor mínimo) }\end{array}$ & $+0,50 \mathrm{~mm}$ & $+0,53 \mathrm{~mm}$ \\
\hline $2^{\circ}$ carregamento & ${ }^{2}$ A vertical & $\begin{array}{c}\text { +1,00 mm } \\
\text { (valor máximo) }\end{array}$ & $+0,97 \mathrm{~mm}$ & $+1,18 \mathrm{~mm}$ \\
\hline $2^{\circ}$ carregamento & C horizontal & $\begin{array}{c}-0,50 \mathrm{~mm} \\
\text { (valor máximo) }\end{array}$ & $-0,50 \mathrm{~mm}$ & $-0,63 \mathrm{~mm}$ \\
\hline
\end{tabular}


A Figura 4.80 representa mais um problema de projeto de mecanismo multi-flexível. Desta vez, apenas o código de otimização elaborado para este trabalho - com a formulação (2.60) implementada - foi empregado para a solução.
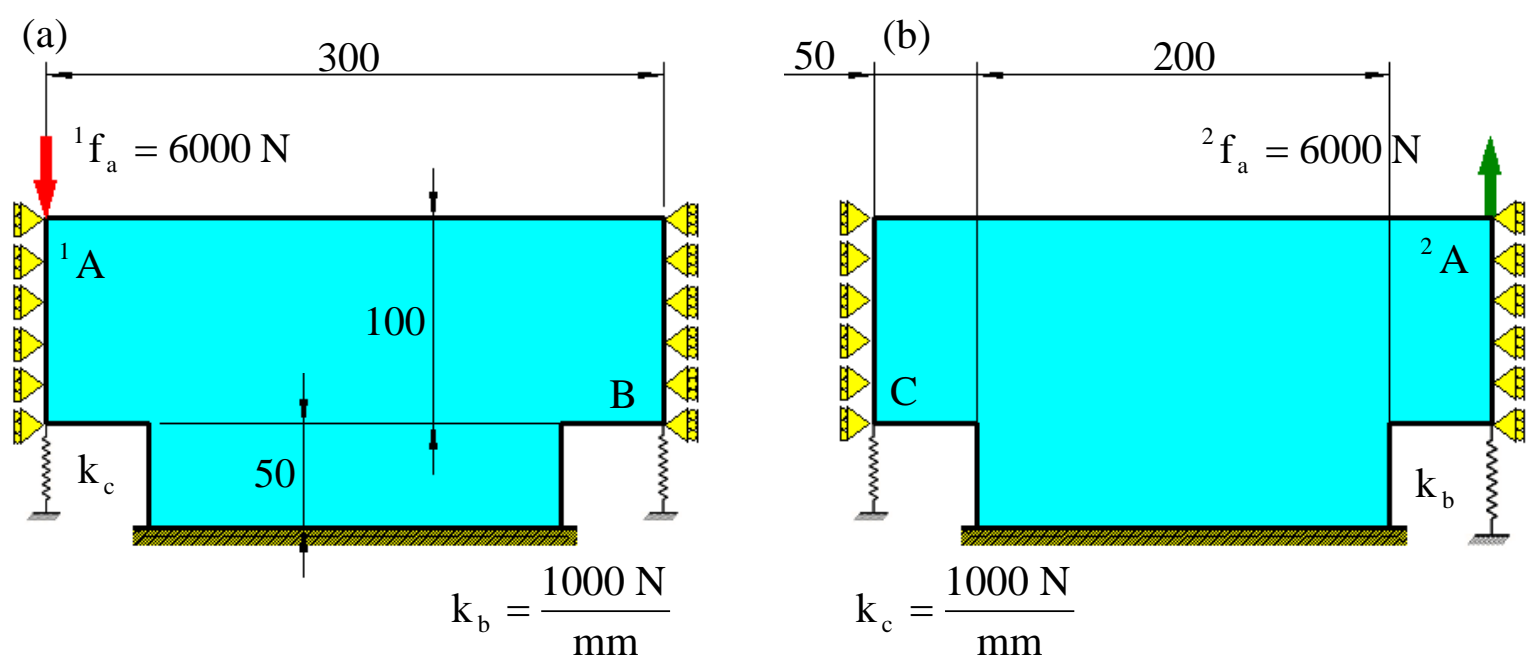

Figura 4.80 - (a) $1^{\circ}$ caso de carregamento do problema de OT; (b) $2^{\circ}$ caso de carregamento do problema de OT.

O aço será o material base a ser distribuído no domínio fixo mostrado pelas Figuras 4.80a e 4.80b. No primeiro caso de carregamento, a força representada por ${ }^{1} \mathrm{f}_{\mathrm{a}}$, cuja intensidade é igual a $6000 \mathrm{~N}$, deverá provocar um deslocamento máximo de $2 \mathrm{~mm}$ no seu ponto de aplicação, bem como um deslocamento mínimo de $1 \mathrm{~mm}$ na tração da mola de rigidez $\mathrm{k}_{\mathrm{b}}$ igual a $1000 \mathrm{~N} / \mathrm{mm}$. No segundo caso de carregamento, a força representada por ${ }^{2} \mathrm{f}_{\mathrm{a}}$, para a qual a intensidade também assume valor igual a $6000 \mathrm{~N}$, provocará o mesmo deslocamento máximo de $2 \mathrm{~mm}$ no seu ponto de aplicação, bem como um deslocamento mínimo de $1 \mathrm{~mm}$ na compressão da mola de rigidez $\mathrm{k}_{\mathrm{c}}$, cujo valor corresponde a $1000 \mathrm{~N} / \mathrm{mm}$. Restringiu-se o material do mecanismo em 50\% do volume do domínio fixo, o qual foi discretizado em 1600 elementos finitos. Adotou-se o valor de 0,15 como estimativa inicial atribuída a todas as variáveis de projeto. O raio de abrangência considerado para o esquema de filtragem corresponde a 5,50 mm. A Figura 4.81 representa a solução do problema. 


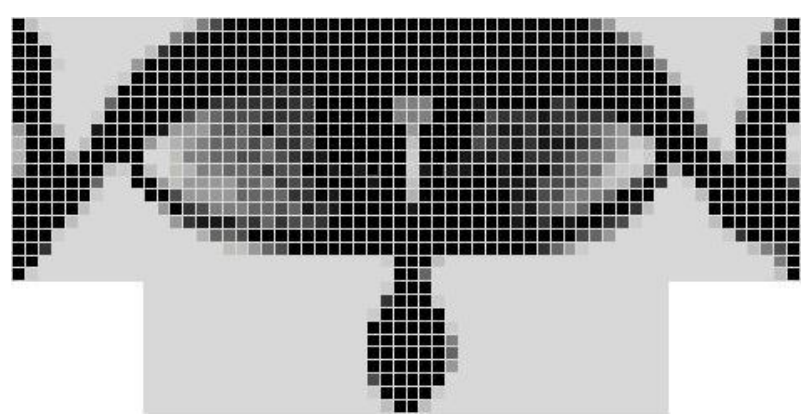

Figura 4.81 - Topologia obtida para o problema de multi-flexibilidade.

As análises estáticas efetuadas sobre o modelo construído a partir da topologia da Figura 4.81 - análises realizadas em ambos os casos de carregamento - fornecem as configurações deformadas representadas pelas Figuras 4.82 e 4.83.

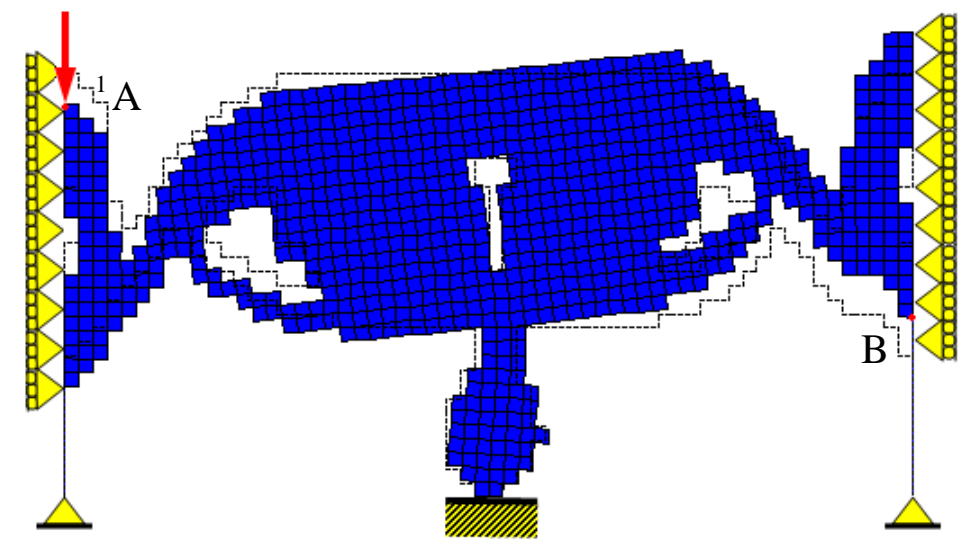

Figura 4.82 - Configuração deformada correspondente ao $1^{\circ}$ caso de carregamento.

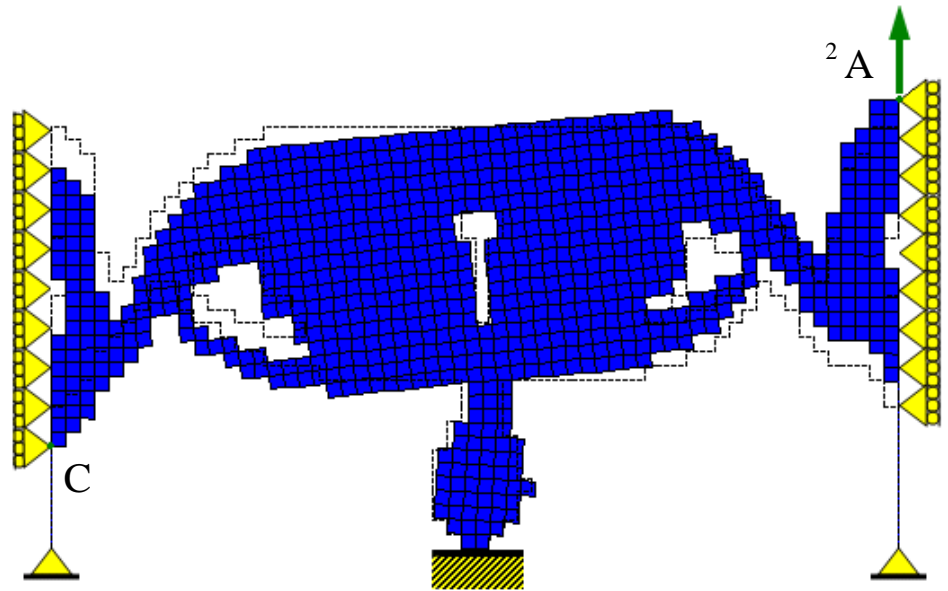

Figura 4.83 - Configuração deformada correspondente ao $2^{\circ}$ caso de carregamento. 
A solução deste problema apresenta uma peculiaridade interessante. Em ambos os casos de carregamento, a forma de se deformar do mecanismo tem o mesmo aspecto, conforme se pode notar através das Figuras 4.82 e 4.83. Os deslocamentos dos pontos ${ }^{1} \mathrm{~A}$, ${ }^{2} \mathrm{~A}$, B e C observados em cada uma das fases de projeto estão registrados na Tabela 4.12.

Tabela 4.12 - Comparação entre os deslocamentos observados nas diferentes etapas de projeto.

\begin{tabular}{|c|c|c|c|c|}
\hline Carregamento & Grau de liberdade & $\begin{array}{c}\text { Restrição imposta ao } \\
\text { deslocamento }\end{array}$ & $\begin{array}{c}\text { Deslocamento no } \\
\text { final do processo de } \\
\text { OT }\end{array}$ & $\begin{array}{c}\text { Deslocamento obtido } \\
\text { na análise do modelo } \\
\text { de EF }\end{array}$ \\
\hline $1^{\circ}$ carregamento & ${ }^{1} \mathrm{~A}$ vertical & $\begin{array}{c}-2,00 \mathrm{~mm} \\
\text { (valor mínimo) }\end{array}$ & $-2,00 \mathrm{~mm}$ & $-2,98 \mathrm{~mm}$ \\
\hline $1^{\circ}$ carregamento & B horizontal & $\begin{array}{c}+1,00 \mathrm{~mm} \\
\text { (valor mínimo) }\end{array}$ & $+1,00 \mathrm{~mm}$ & $+1,78 \mathrm{~mm}$ \\
\hline $2^{\circ}$ carregamento & ${ }^{2}$ A vertical & $\begin{array}{c}\text { +2,00 mm } \\
\text { (valor máximo) }\end{array}$ & $+1,64 \mathrm{~mm}$ & $+2,48 \mathrm{~mm}$ \\
\hline $2^{\circ}$ carregamento & C horizontal & $\begin{array}{c}-1,00 \mathrm{~mm} \\
\text { (valor máximo) }\end{array}$ & $-1,00 \mathrm{~mm}$ & $-1,78 \mathrm{~mm}$ \\
\hline
\end{tabular}




\section{CONCLUSÃO}

\subsection{CONCLUSÕES E OBSERVAÇÕES FINAIS}

Foi desenvolvido um código computacional para projetar mecanismos flexíveis menos sujeitos à ocorrência de um determinado tipo de instabilidade numérica denominada “dobradiça”. Para tanto, a maximização da energia de deformação em regiões preenchidas com o material base demonstrou ser uma estratégia viável para minimizar o problema. Conforme foi mostrado, a formulação implementada não requer a aplicação de recursos numéricos sofisticados ou restrições locais específicas, o que reduz os custos computacionais. Investigou-se a variação de determinados parâmetros do processo de otimização topológica, verificando-se, entre outras coisas, que as topologias têm flexibilidade melhor distribuída na medida em que se reduz a quantidade de material disponível à otimização. No entanto, para maiores quantidades de material, podem surgir regiões cinzas capazes de servir como pontos de concentração da flexibilidade do mecanismo. Demonstrou-se que estas regiões acinzentadas podem ser eliminadas através da alteração dos parâmetros de filtragem, melhorando a definição do contorno da topologia na região sem que surjam as “dobradiças”. Ainda que alguma “dobradiça” apareça nestas condições, um refinamento da malha de elementos finitos é capaz de removê-la. As mudanças nas restrições de deslocamento e na rigidez da mola, que é utilizada para representar a peça de trabalho, também alteram a topologia do mecanismo flexível.

Embora os resultados não tenham sido conclusivos, testou-se a aplicação da maximização da energia de deformação no projeto de estruturas rígidas. As topologias encontradas são soluções sensivelmente diferentes daquelas tradicionalmente obtidas por meio da formulação baseada na minimização da flexibilidade média com restrição de volume de material.

A maximização da energia de deformação mostrou-se adequada também à multiflexilidade. Estendeu-se a formulação aplicável à obtenção de mecanismos flexíveis simples ao projeto de mecanismos multi-flexíveis, nos quais foram observados os mesmos resultados em relação às “dobradiças”, o que demonstra a generalidade da formulação. 


\subsection{SUGESTÕES PARA FUTUROS TRABALHOS}

Algumas questões relacionadas a esta dissertação podem servir como objeto de discussão para futuros trabalhos. Assim, como exemplo, um estudo mais profundo acerca das topologias das estruturas rígidas obtidas a partir da maximização da energia de deformação deve fornecer maiores subsídios à compreensão da formulação discutida neste trabalho.

Com respeito à questão das “dobradiças", foco principal deste texto, outras possibilidades são passíveis de investigação. Pode-se, por exemplo, desenvolver esquemas numéricos capazes de aglutinar elementos finitos triangulares em "super-elementos" hexagonais. Como se sabe, hexágonos adjacentes mantêm sempre uma aresta em comum, o que eliminaria definitivamente a ocorrência das “dobradiças”. Caso esta alternativa se mostrasse plausível, seria recomendável investigar as características próprias das topologias obtidas a partir de figuras hexagonais, avaliando-se, também, os custos computacionais inerentes à aplicação do esquema numérico.

Acredita-se que, com base em toda pesquisa e investigação, este trabalho, baseado na formulação proposta por Cardoso e Fonseca (2004), consolide a idéia de maximizar a energia de deformação como uma alternativa viável para o projeto mecanismos flexíveis menos suscetíveis às “dobradiças”. 


\section{REFERÊNCIAS}

ALBUQUERQUE, F., 1991, “Programando em Linguagem C, C++ e Turbo C++”, $1^{\text {a }}$ Edição, Berkeley EBRAS Editora Brasileira.

ALLAIRE, G., 2002, “Shape Optimization by the Homogenization Method”, Springer Verlag.

ALLAIRE, G.; AUBRY, S., 1999, “On Optimal Microstructures for a Plane Shape Optimization Problem”, Structural and Multidisciplinary Optimization, 17, pp. 86-94.

ALLAIRE, G.; KOHN, R.V., 1993, “Optimal Design for Minimum Weight and Compliance in Plane Stress Using Extremal Microstructures”, European Journal of Mechanics A. Solids, 12, pp. 839-878.

AMBROSIO, L.; BUTTAZZO, G., 1993, “An Optimal Design Problem with Perimeter Penalization”, Calculus and Variations and Partial Differential Equations, 1, pp. 55-69.

ANANTHASURESH, G. K.; KOTA, S., 1995, “Designing Compliant Mechanisms”, ASME Mechanical Engineering, 117, pp. 93-96.

ANANTHASURESH, G. K.; KOTA, S.; GIANCHANDANI, Y., 1994, “A Methodical Approach to the Design of Compliant Micromechanisms", Solid-State Sensor and Actuator Workshop, pp. 189-192.

ANDERSEN, M., 1997, “Shape Optimization of Compliant Mechanisms”, Master's Thesis, Technical University of Denmark, Department of Solid Mechanics (in Danish).

BAHIA, M. T., 2005, “Otimização Topológica Aplicada ao Projeto de Mecanismos Flexíveis”, Dissertação de Mestrado, Universidade Federal de Santa Catarina.

BATHE, K. J., 1996, “Finite Elements Procedures”, Prentice Hall, New Jersey.

BENDSØE, M. P., 1989, “Optimal Shape Design as a Material Distribution Problem”, Structural and Multidisciplinary Optimization, 1, pp. 192-202.

BENDSØE, M. P., 1995, “Optimization of Structural Topology, Shape and Material”, Springer Verlag.

BENDSØE, M. P.; KIKUCHI, N., 1988, "Generating Optimal Topologies in Structural Design Using a Homogenization Method”, Computer Methods in Applied Mechanics and Engineering, 71, pp. 197-224. 
BENDSØE, M. P.; SIGMUND, O., 1999, "Material Interpolations Schemes in Topology Optimization”, Archive of Applied Mechanics, 69, pp. 635-654.

BENDSØE, M. P.; SIGMUND, O., 2003, “Topology Optimization: Theory, Methods and Applications”, Springer Verlag, Berlin Heidelberg.

BENDSØE, M. P.; SOARES, C. A. M., 1992, “Topology Design of Structures”, NATO ASI Series - Series E: Applied Sciences - Vol. 227.

BOURDIN, B., 2001, “Filters in Topology Optimization”, International Journal for Numerical Methods in Engineering, 50 (9), pp. 2143-2158.

BRAESS, D., 2001, "Finite Elements: Theory, Fast Solvers, and Applications in Solid Mechanics”, 2nd Edition, Cambridge.

BREMICKER, M., Chirehdast, M., Kikuchi, N., Papalambros, P. Y., 1991, "Integrated Topology and Shape Optimization in Structural Design", Mechanics of Structures and Machines, 19, pp. 551-587.

BRUNS, T. E.; TORTORELLI, D. A., 2001, “Topology Optimization of Non-Linear Elastic Structures and Compliant Mechanisms”, Computer Methods in Applied Mechanics and Engineering, 190(26-27), pp. 3443-3459.

CANFIELD, S.; FRECKER, M. I., 2000, “Topology Optimization of Compliant Mechanical Amplifiers for Piezoelectric Actuators”, Structural and Multidisciplinary Optimization, 20, pp. 269-279.

CARDOSO, E. L., 2000, "Controle de Complexidade na Otimização Topológica de Estruturas Contínuas”, Dissertação de Mestrado, Escola de Engenharia da Universidade Federal do Rio Grande do Sul.

CARDOSO, E. L.; FONSECA, J. S. O., 1999, "Intermediate Density Reduction and Complexity Control in the Topology Optimization”, 20th Iberian Latin-American Congress on Computational Methods in Engineering (CILAMCE’99), Brasil.

CARDOSO, E. L.; FONSECA, J. S. O., 2003, "Complexity Control in the Topology Optimization of Continuum Structures", Journal of the Brazilian Society of Mechanical Sciences and Engineering, 25, pp. 293-301.

CARDOSO, E. L.; FONSECA, J. S. O., 2004, “Strain Energy Maximization Approach to the Design of Fully Compliant Mechanisms Using Topology Optimization”, Latin American Journal of Solids and Structures, 1, pp. 263-275. 
CHEN, B. C.; SILVA, E. C. N.; KIKUCHI, N., 2001, “Advances in Computational Design and Optimization with Application to MEMS", International Journal for Numerical Methods in Engineering, 52, pp. 23-62.

CHENG, K. T., 1981, “On Non-Smoothness in Optimal Design of Solid, Elastic Plates”, International Journal of Solids and Structures, 17, pp. 795-810.

CHENG, K. T.; OLHOFF, N., 1981, “An Investigation Concerning Optimal Design of Solid Elastic Plates”, International Journal of Solids and Structures, 17, pp. 305-323.

DANTZIG, G. B., 1963, “Linear Programming and Extensions”, Princeton University Press.

DÍAZ, A. R.; SIGMUND, O., 1995, “Checkerboard Patterns in Layout Optimization”, Structural and Multidisciplinary Optimization, 10, pp. 40-45.

DUYSINX, P.; BENDSØE, M. P., 1998, “Topology Optimization of Continuum Structures with Local Stress Constraints”, International Journal for Numerical Methods in Engineering, 43 (8), pp. 1453-1478.

FRECKER, M. I.; KIKUCHI, N.; KOTA, S., 1996, "Optimal Synthesis of Compliant Mechanisms to Satisfy Kinematic and Structural Requirements - Preliminary Results", Proceedings of the 1996 ASME Design Engineering Technical Conferences and Computers in Engineering Conference, Irvine, CA, USA, August 18-22, pp. 177-192.

FUKUSHIMA, J.; SUZUKI, K.; KIKUCHI, N., 1991, “Applications to Car Bodies: Generalized Layout Design of Three-Dimensional Shells”, Optimization of Large Structural Systems; Proceedings of the NATO/DFG Advanced Study Institute, Berchtesgaden, Germany, Sept. 23-Oct. 4, 1991. Vol. 1 (A93-54501 24-39), p. 177-191.

GUEDES, J. M.; KIKUCHI, N., 1990, "Preprocessing and Postprocessing for Materials Based on the Homogenization Method with Adaptive Finite Element Methods”, Computer Methods in Applied Mechanics Engineering, 83, pp. 143-198.

HABER, R. B.; JOG, C. S.; BENDSØE, M. P., 1996, "A New Approach to VariableTopology Shape Design Using a Constraint on Perimeter”, Structural and Multidisciplinary Optimization, 11, pp. 1-12.

HAFTKA, R. T.; GRANDHI, R.V., 1986, "Structural Shape Optimization - A Survey", Computer Methods in Applied Mechanics and Engineering, 57, pp. 91-106.

HAFTKA, R. T.; GÜRDAL, Z.; KAMAT, M. P., 1990, "Elements of Structural Optimization”, Kluwer Academic Publishers, Boston. 
HASHIN, Z.; SHTRIKMAN, S., 1963, “A Variational Approach to the Theory of the Elastic Behavior of Multiphase Materials", Journal of the Mechanics and Physics of Solids, 11, pp. 127-140.

HASSANI, B.; HINTON, E., 1998, “A Review of Homogenization and Topology Optimization, I - Homogenization Theory for Media with Periodic Structure”, Computers and Structures, 69, pp. 707-717.

HER, I.; MIDHA, A., 1987, “A Compliance Number Concept for Compliant Mechanisms and Type Synthesis", Journal of Mechanisms, Transmissions, and Automation in Design, Transaction of the ASME, 109, pp. 348-355.

HETRICK, J. A.; KIKUCHI, N.; KOTA, S., 1999, "Robustness of Compliant Mechanism Topology Optimization Formulations”, Mathematics and Control in Smart Structures, Proceedings of the Meeting, Newport Beach, CA, USA, March 1-4, pp. 244-254.

HOWELL, L. L., 2001, “Compliant Mechanisms”, John Wiley \& Sons.

HOWELL, L. L.; MIDHA, A., 1994, “A Method for the Design of Compliant Mechanisms with Small-Length Flexural Pivots”, Journal of Mechanical Design, Transaction of the ASME, 116, pp. 280-290.

HOWELL, L. L.; MIDHA, A., 1996, “A Loop-closure Theory for the Analysis and Synthesis of Compliant Mechanisms", Journal of Mechanical Design, Transaction of the ASME, 118, pp. 121-125.

HUANG, N. C., 1971, "On principle of Stationary Mutual Complementary Energy and its Application to Optimal Structural Design", Journal of Applied Mathematics and Physics, ZAMP 22, pp. 608-620.

JOG., C. S.; HABER, R. B., 1996, “Stability of Finite Element Models for Distributed Parameter Optimization and Topology Design”, Computer Methods in Applied Mechanics and Engineering, 130, pp. 203-226.

JONSMANN, J.; SIGMUND, O.; BOUWSTRA, S., 1999, "Compliant Electro-Thermal Microactuators”, Proceedings of MEMS 99, pp. 588-593.

KARMARKAR, N., 1984, “A New Polynomial-Time Algorithm for Linear Programming”, Combinatorica, 4, pp. 373-395.

KIM, C. J., PISANO, A. P., MULLER, R.S., 1992, "Silicon-processed Overhanging Microgripper”, Journal of Microelectromechanical Systems, 1, pp. 31-36. 
KOTA, S.; Joo, J.; Li, Z.; Rodgers, S. M.; Sniegowski, J., 2001. "Design of Compliant Mechanisms: Applications to MEMS”, Analog Integrated Circuits and Signal Processing, 29, pp. 7-15.

LARSEN, U. D.; Sigmund, O.; Bouwstra, S., 1997, "Design and Fabrication of Compliant Micromechanisms and Structures with Negative Poisson's Ratio”, Journal of Microelectromechanical Systems, 6(2), pp. 99-106.

LAU, G. K.; DU, H.; LIM, M. K., 2001, "Use of Functional Specifications as Objective Functions in Topology Optimization of Compliant Mechanism", Computer Methods in Applied Mechanics And Engineering, 190, pp. 4421-4433.

LIMA, C. R., 2002, "Projeto de Mecanismos Flexíveis Usando o Método de Otimização Topológica”, Dissertação de Mestrado, Escola Politécnica da Universidade de São Paulo, Departamento de Engenharia Mecatrônica.

LOU, Z., Chen, L., Yang, J., Zhang, Y., Abdel-Malek, K., 2005, “Compliant Mechanism Design Using Multi-Objective Topology Optimization Scheme of Continuum Structures”, Structural and Multidisciplinary Optimization, 30, pp. 142-154.

MA, Z. D.; KIKUCHI, N.; CHENG, H.C., 1995, “Topological Design for Vibrating Structures”, Computer Methods in Applied Mechanics and Engineering, 121. 1-4, pp. 259280.

MICHELL, A. G. M., 1904, “The Limit of Economy of Material in Frame Structures”, Philosophical Magazine, 8(6), pp. 589-597.

MIDHA, A.; NORTON, T. W.; HOWELL, L. L., 1992, "On the Nomenclature and Classification of Compliant Mechanisms: Abstraction of Mechanisms and Mechanism Synthesis Problems”, Flexible Mechanisms, Dynamics and Analysis, ASME 1992 Design Technical Conferences, 22nd Biennial Mechanisms Conferences, Scottsdale, Arizona, USA, Vol. 47, pp. 13-16.

MLEJNEK, H. P.; SCHIRRMACHER, R., 1993, “An Engineer's Approach to Optimal Material Distribution and Shape Finding", Computer Methods in Applied Mechanics and Engineering, 106, pp. 1-26.

NEVES, M. M.; RODRIGUES, H.; GUEDES, J. M., 1995, “Generalized Topology Design of Structures with a Buckling Load Criterion”, Structural and Multidisciplinary Optimization, 10(2), pp. 71-78.

NIORDSON, F., 1983, "Optimal Design of Plates with a Constraint on the Slope of the Thickness Function”, International Journal of Solids and Structures, 19, pp. 141-151. 
NISHIWAKI, S.; Frecker, M. I.; Min, S.; Kikuchi, N., 1998, "Topology Optimization of Compliant Mechanisms Using the Homogenization Method", International Journal for Numerical Methods in Engineering, 42, pp. 535-559.

NISHIWAKI, S.; Min, S.; Yoo, J.; Kikuchi, N., 2001, “Optimal Structural Design Considering Flexibility”, Computer Methods in Applied Mechanics and Engineering, 190, pp. 4457-4504.

ODEN, J. T.; KIKUCHI, N.; SONG, Y. J., 1982, "Penalty-Finite Element Methods for the Analysis of Stokesian Flows", Computer Methods in Applied Mechanics and Engineering, 31, pp. 297-329.

PARK., Y. K., 1995, “Extensions of Optimal Layout Design Using Homogenization Method”, Ph.D. Dissertation, University of Michigan.

PEDERSEN, C. B. W.; BUHL, T., SIGMUND, O., 2001, "Topology Synthesis of Large Displacement Compliant Mechanisms", International Journal for Numerical Methods in Engineering, 50, pp. 2683-2705.

PETERSEN, K. E., 1982, "Silicon as a Mechanical Material”, Proceedings of the IEEE, 70(5), pp. 420-457.

PETERSSON, J.; SIGMUND, O., 1998, “Slope Constrained Topology Optimization”, International Journal for Numerical Methods in Engineering, 41(8), pp. 1417-1434.

POULSEN, T. A., 2001, "A New Scheme for Imposing a Minimum Length Scale in Topology Optimization”, Danish Center for Applied Mathematics and Mechanics, DCAMM Report No. 663.

POULSEN, T. A., 2002, “A simple Scheme to Prevent Checkerboard Patterns and One-Node Connected Hinges in Topology Optimization”, Structural and Multidisciplinary Optimization, 24, pp. 396-399.

RAHMATALLA, S., SWAN, C. C., 2005, "Sparse Monolithic Compliant Mechanisms Using Continuum Structural Topology Optimization” , International Journal for Numerical Methods in Engineering, 62, pp. 1579-1605.

RIETZ, A., 2001, "Sufficiency of a Finite Exponent in SIMP (Power Law) Methods", Structural and Multidisciplinary Optimization, 21, pp. 159-163.

ROCKAFELLAR, R., T., 1997, “Convex Analysis”, Princeton Landmarks in Mathematics, New Jersey. 
ROZVANY, G. I. N., 1989, “Structural Design via Optimality Criteria”, Kluwer Academic Publishers Group, Dordrecht.

ROZVANY, G. I. N.; BENDSØE, M. P.; KIRSCH, U., 1995, "Layout Optimization of Structures”, Applied Mechanics Reviews, 48, pp. 41-119.

ROZVANY, G. I. N.; ZHOU, M., 1991, “The COC Algorithm, Part I: Cross Section Optimization or Sizing”, Computer Methods in Applied Mechanics and Engineering, 89, pp 281-308.

ROZVANY, G. I. N., ZHOU, M., BIRKER, T., 1992, "Generalized Shape Optimization without Homogenization”, Structural and Multidisciplinary Optimization, 4(3-4), pp. 250252.

SHIELD, R. T.; PRAGER, W., 1970, “Optimal Structural Design for Given Deflection”, Journal of Applied Mathematics and Phisics, ZAMP 21, pp. 513-523.

SIGMUND, O., 1994, “Design of Material Structures Using Topology Optimization”, PhD thesis, Department of Solid Mechanics, Technical University of Denmark.

SIGMUND, O., 1997, "On the Design of Compliant Mechanisms Using Topology Optimization”, Mechanics of Structures and Machines, 25(4), pp. 495-526.

SIGMUND, O., 2001a, “A 99 Line Topology Optimization Code Written in MATLAB”, Structural and Multidisciplinary Optimization, 21, pp.120-127.

SIGMUND, O., 2001b, "Design of Multiphysics Actuators Using Topology Optimization Part I: One-Material Structures”, Computer Methods in Applied Mechanics and Engineering, 190(49-50), pp. 6577-6604.

SIGMUND, O., 2001c, "Design of Multiphysics Actuators Using Topology Optimization Part II: Two-Material Structures”, Computer Methods in Applied Mechanics and Engineering, 190(49-50), pp. 6605-6627.

SILVA, E. C. N.; FONSECA, J. S. O.; KIKUCHI, N., 1997, “Optimal Design of Piezoelectric Microstructures”, Computacional Mechanics, 19(5), pp. 397-410.

SILVA, E. C. N.; FONSECA, J. S. O.; KIKUCHI, N., 1998, “Optimal Design of Periodic Piezocomposites”, Computer Methods in Applied Mechanics and Engineering 159(2), pp. 4977.

SILVA, E. C. N.; Nishiwaki, S.; Fonseca, J. S. O.; Kikuchi, N., 1999, “Optimization Methods Applied to Material and Flextensional Actuator Design Using the Homogenization Method", Computer Methods in Applied Mechanics and Engineering, 172, pp. 241-271. 
SILVA, E. C. N.; NISHIWAKI, S.; KIKUCHI, N., 2000, “Topology Optimization Design of Flextensional Actuators”, IEEE Transactions on Ultrasonics, Ferroelectrics and Frequency Control, 47, pp. 657-671.

SUZUKI, K.; KIKUCHI, N., 1991, “A Homogenization Method for Shape and Topology Optimization”, Computer Methods in Applied Mechanics and Engineering, 93, pp. 291-318.

SVANBERG, K., 1987, "The Method of Moving Asymptotes - A New Method for Structural Optimization”, International Journal for Numerical Methods in Engineering, 24, 359-373.

SWAN, C. C.; KOSAKA, I., 1997, "Voigt-Reuss Topology Optimization for Structures with Linear Elastic Material Behaviors”, International Journal of Numerical Methods in Engineering, 40, 1, pp. 3033-3057.

TAI, K.; CHEE, T. H., 2000, "Design of Structures and Compliant Mechanisms by Evolutionary Optimization of Morphological Representations of Topology”, Transactions of the ASME 122: 560566.

THOMAS, H. L.; VANDERPLAATS, G. N.; SHYY, Y. K., 1992, “A Study of Move Limit Adjustment Strategies in the Approximation Concepts Approach to Structural Synthesis", AIAA-VMA Engineering, pp. 507-512.

THOMSEN, J., 1992, “Topology Optimization of Structures Composed of One or Two Materials”, Structural Optimization, 5, pp. 108-115.

VANDERPLAATS, G. N., 1984, "Numerical Optimization Techniques for Engineering Design: with Applications”. McGraw-Hill.

YANG, R. J.; CHUANG, C. H., 1994, "Optimal Topology Design Using Linear Programming”, Computers and Structures, 52(2), pp. 265-275.

YIN, L.; ANANTHASURESH, G. K., 2001, “Topology Optimization of Compliant Mechanisms with Multiple Materials Using a Peak Function Material Interpolation Scheme”, Structural and Multisciplinary Optimization, 23(1), pp. 49-62.

YOON, G. H.; Kim, Y. Y.; Bendsøe, M. P.; Sigmund, O., 2004, "Hinge-free Topology Optimization with Embedded Translation-invariant Differentiable Wavelet Shrinkage", Structural and Multisciplinary Optimization, 27, pp. 139-150. 


\section{APÊNDICE A - Aferição da rotina de elementos finitos}

Conforme demonstrado ao longo do texto, o código computacional desenvolvido para o projeto de mecanismos flexíveis contém uma rotina de elementos finitos que calcula o campo dos deslocamentos associados ao domínio fixo de projeto. Assim, este apêndice tem o objetivo de demonstrar que a implementação numérica do MEF realizada neste trabalho gera resultados com um nível de precisão adequado. Para isso, adotou-se um problema de análise estática cuja solução numérica foi obtida, primeiramente, através do software comercial ANSYS versão 10.0 e, posteriormente, através da rotina de elementos finitos implementada no software elaborado. Os resultados foram comparados conforme está indicado na Tabela A.1. O problema proposto corresponde a uma estrutura bidimensional submetida ao carregamento representado pela Figura A.1.

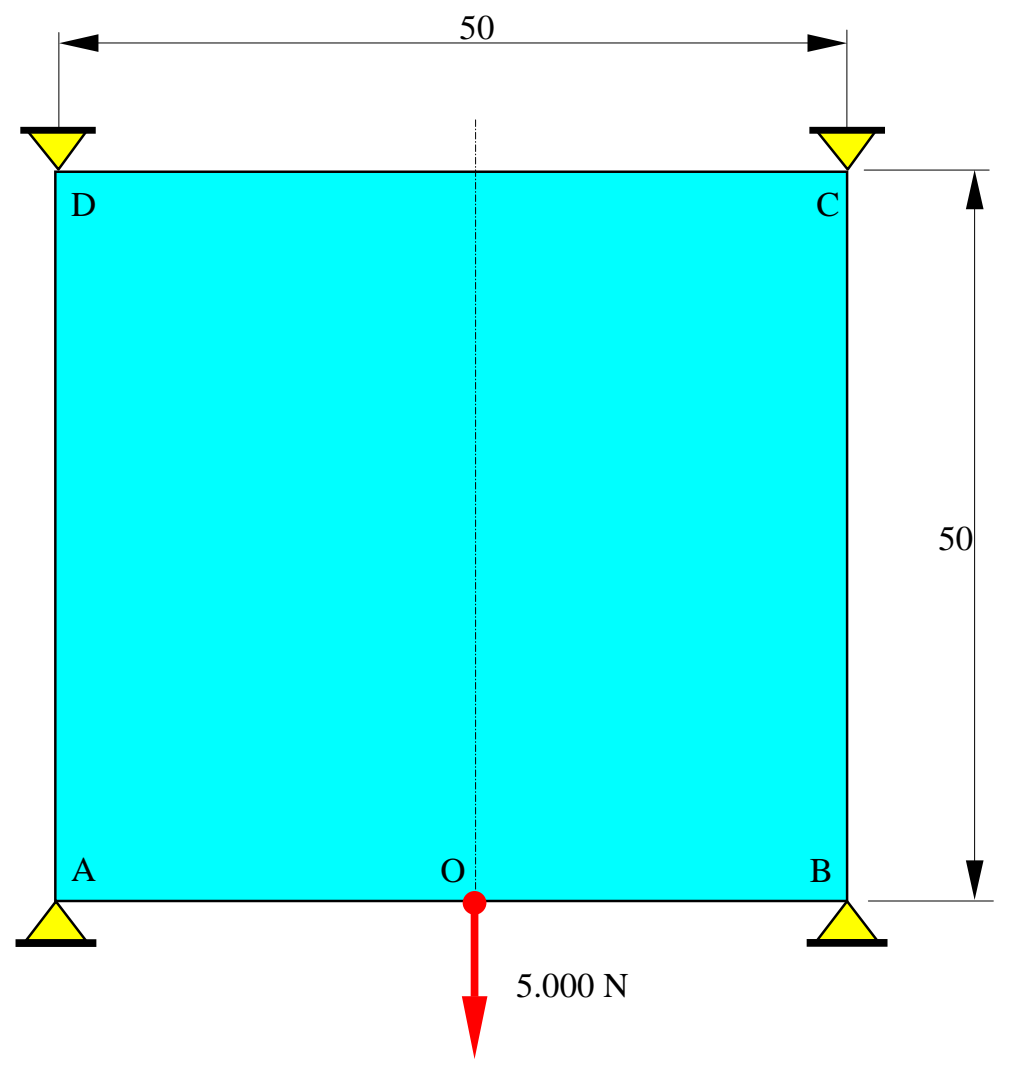

Figura A.1 - Estrutura bidimensional de espessura constante igual a $1 \mathrm{~mm}$.

Adotou-se o módulo de elasticidade longitudinal (E) e a razão de Poisson ( $v$ ) correspondentes a $2,1 \times 10^{11} \mathrm{~N} / \mathrm{m}^{2}$ e 0,3 , respectivamente. 
A estrutura foi discretizada em 2500 elementos finitos quadrados de quatro nós ajustados ao estado plano de tensões. Com isso, o segmento $\overline{\mathrm{AO}}$ da Figura A.1 passou a conter 26 nós da malha de elementos finitos. A Figura A.2 representa a configuração deformada deste segmento.

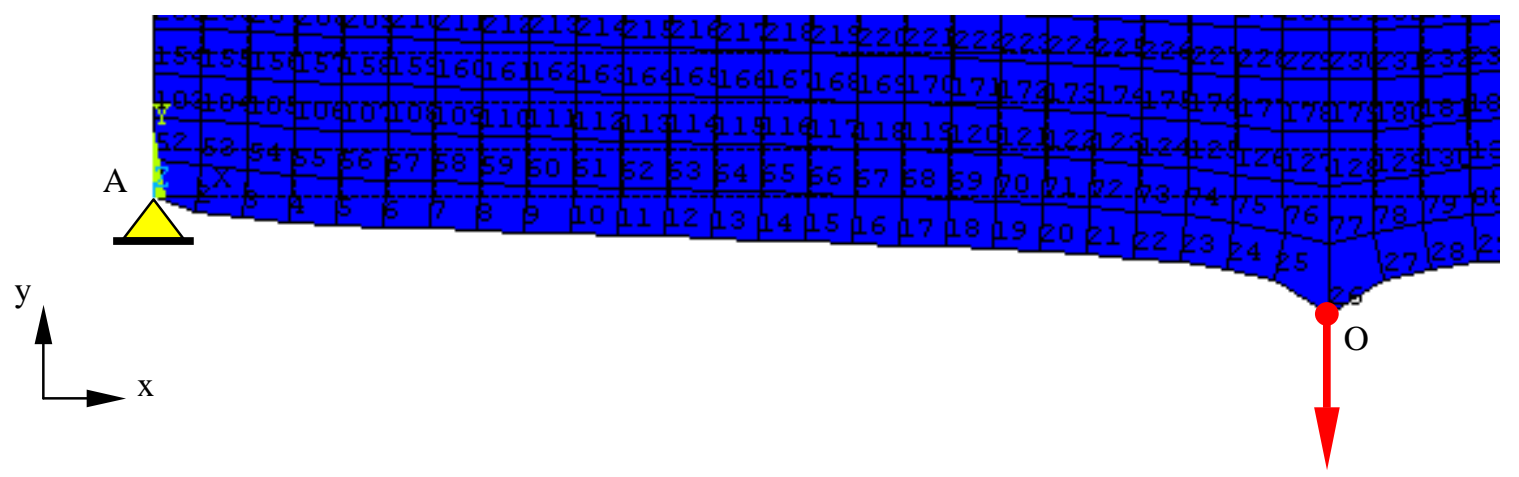

Figura A.2 - Configuração deformada do segmento $\overline{\mathrm{AO}}$.

Para os nós numerados de 1 a 26, a Tabela A.1 compara os deslocamentos verticais obtidos através do software comercial ANSYS 10.0 com aqueles deslocamentos correspondentes determinados pela rotina de MEF implementada no código de projeto de mecanismos flexíveis.

Tabela A.1 - Comparação entre os deslocamentos verticais obtidos a partir do ANSYS 10.0 e da rotina de MEF.

\begin{tabular}{cccc}
\hline Numeração do nó & $\begin{array}{c}\text { Deslocamento obtido com } \\
\text { o software ANSYS 10.0 }\end{array}$ & $\begin{array}{c}\text { Deslocamento obtido com } \\
\text { a rotina implementada }\end{array}$ & $\begin{array}{c}\text { Erro - variação percentual } \\
\text { em módulo }\end{array}$ \\
\hline 1 & 0 & 0 & - \\
2 & $-0.016298 \mathrm{~mm}$ & $-0.016294 \mathrm{~mm}$ & $0,024543 \%$ \\
3 & $-0.021220 \mathrm{~mm}$ & $-0.021214 \mathrm{~mm}$ & $0,028275 \%$ \\
4 & $-0.024828 \mathrm{~mm}$ & $-0.024822 \mathrm{~mm}$ & $0,024166 \%$ \\
5 & $-0.027430 \mathrm{~mm}$ & $-0.027423 \mathrm{~mm}$ & $0,025520 \%$ \\
6 & $-0.029571 \mathrm{~mm}$ & $-0.029563 \mathrm{~mm}$ & $0,027054 \%$ \\
7 & $-0.031419 \mathrm{~mm}$ & $-0.031412 \mathrm{~mm}$ & $0,022280 \%$ \\
8 & $-0.033082 \mathrm{~mm}$ & $-0.033077 \mathrm{~mm}$ & $0,015114 \%$ \\
9 & $-0.034627 \mathrm{~mm}$ & $-0.034622 \mathrm{~mm}$ & $0,014440 \%$ \\
10 & $-0.036099 \mathrm{~mm}$ & $-0.036095 \mathrm{~mm}$ & $0,011081 \%$ \\
11 & $-0.037533 \mathrm{~mm}$ & $-0.037529 \mathrm{~mm}$ & $0,010657 \%$ \\
12 & $-0.038956 \mathrm{~mm}$ & $-0.038953 \mathrm{~mm}$ & $0,007701 \%$ \\
13 & $-0.040395 \mathrm{~mm}$ & $-0.040393 \mathrm{~mm}$ & $0,004951 \%$ \\
14 & $-0.041873 \mathrm{~mm}$ & $-0.041873 \mathrm{~mm}$ & $0,000000 \%$ \\
15 & $-0.043414 \mathrm{~mm}$ & $-0.043416 \mathrm{~mm}$ & $0,004607 \%$ \\
16 & $-0.045045 \mathrm{~mm}$ & $-0.045048 \mathrm{~mm}$ & $0,006660 \%$ \\
17 & $-0.046799 \mathrm{~mm}$ & $-0.046801 \mathrm{~mm}$ & $0,004274 \%$ \\
18 & $-0.048715 \mathrm{~mm}$ & $-0.048717 \mathrm{~mm}$ & $0,004106 \%$ \\
19 & $-0.050848 \mathrm{~mm}$ & $-0.050849 \mathrm{~mm}$ & $0,001967 \%$ \\
20 & $-0.053273 \mathrm{~mm}$ & $-0.053274 \mathrm{~mm}$ & $0,001877 \%$ \\
21 & $-0.056105 \mathrm{~mm}$ & $-0.056106 \mathrm{~mm}$ & $0,001782 \%$ \\
22 & $-0.059545 \mathrm{~mm}$ & $-0.059546 \mathrm{~mm}$ & $0,001679 \%$ \\
24 & $-0.063910 \mathrm{~mm}$ & $-0.063911 \mathrm{~mm}$ & $0,001565 \%$ \\
& $-0.070270 \mathrm{~mm}$ & $-0.070270 \mathrm{~mm}$ & $0,000000 \%$
\end{tabular}


Na Tabela A.1, os erros, calculados com base nos valores obtidos pelo software comercial ANSYS 10.0, demonstram que a rotina de MEF implementada fornece resultados adequados para o cálculo do campo dos deslocamentos. 